\title{
Photochemistry of small molecules
}

\author{
Dissertation \\ by \\ Britta Niederjohann
}

Department of Physics, University of Bielefeld

August 2004 

'Photochemistry deals with physical and chemical change initiated by the interaction of light with a molecule.'

Hideo Okabe, 1978 



\section{Contents}

1 Introduction to Photochemistry 4

2 Photodissociation and reaction dynamics $\quad 6$

2.1 Photodissociation and reactive collisions . . . . . . . . . 6

2.2 Photodissociation kinetics . . . . . . . . . . . . 9

2.3 Angular distribution of the fragments . . . . . . . . . . . 10

3 Experimental Set-up $\quad 13$

3.1 The vacuum apparatus . . . . . . . . . . . . . . . . . 13

3.2 The laser systems . . . . . . . . . . . . . . . . . . . . . 14

3.2.1 The VUV laser . . . . . . . . . . . . . . 16

3.3 Delay control . . . . . . . . . . . . . . . . . . 17

I OH-beam 19

4 Introduction to the OH-Source 20

5 Experimental Set-Up for $\mathrm{OH}$ detection 26

5.1 LIF set-up . . . . . . . . . . . . . . . . 26

5.2 Hydrogen detection . . . . . . . . . . . . . . . . . 28

5.2.1 Laser adjustment . . . . . . . . . . . . . . . . 29

6 Estimate of the total Signal $\quad 31$

7 OH Precursor $\quad 35$

7.1 Precursor $\mathrm{H}_{2} \mathrm{O}_{2} \ldots \ldots \ldots$. . . . . . . . . . . . 35

$7.2 \mathrm{H}_{2} \mathrm{O}_{2}$ from a pulsed valve . . . . . . . . . . . . 36

7.2.1 Preparation of $\mathrm{H}_{2} \mathrm{O}_{2}$ from an aqueous solution . . . . . 37

7.2.2 Preparation of $\mathrm{H}_{2} \mathrm{O}_{2}$ from urea/hydrogen peroxide com-

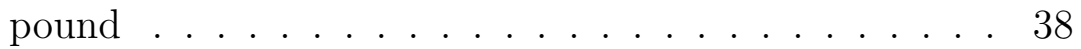

7.2.3 $\mathrm{H}_{2} \mathrm{O}_{2}$ to $\mathrm{HO}_{2}+\mathrm{H}$ channel . . . . . . . . . . . . 38 
7.2.4 LIF from $\mathrm{H}_{2} \mathrm{O}_{2}$. . . . . . . . . . . . . . . . . . 40

7.3 Results from $\mathrm{H}_{2} \mathrm{O}_{2}$ with an effusive beam . . . . . . . . . . . 41

7.4 Precursor $\mathrm{HNO}_{3} \ldots \ldots \ldots$. . . . . . . . . . . . 44

8 Summary and Outlook $\quad 48$

II $\mathrm{I}_{2}$ and $\mathrm{I}_{2}^{+}$Dissociation $\quad 53$

9 Aims of this Work $\quad 54$

$\begin{array}{ll}10 \text { Introduction to Iodine Potentials } & 57\end{array}$

11 Velocity Map Imaging $\quad 62$

11.1 Principle of velocity map imaging . . . . . . . . . . . . 62

11.2 Event counting . . . . . . . . . . . . . . . . . . . . . 65 65

11.3 Abel inversion and BASEX expansion . . . . . . . . . . . . 67

11.4 Calibration of the images . . . . . . . . . . . . . . 71

11.5 Determination of the $\beta$-parameter . . . . . . . . . 75

12 Experimental Setup $\quad 77$

12.1 Detector and ion lens . . . . . . . . . . . . . . . . . . . . . . . . . . 77

12.2 The $\mathrm{I}_{2}$ molecular beam . . . . . . . . . . . . . . . 79

13 Mass Spectrum and Photoelectrons 81

13.1 Mass spectrum ................. . . . 81

13.2 Photoelectron images . . . . . . . . . . . . . . . . . . . . . . . . . . . . 83

13.2.1 $\mathrm{X}^{2} \Pi_{g}$ states ................ . . 86

$13.2 .2 \quad \mathrm{~A}^{2} \Pi_{u}$ states . . . . . . . . . . . . . . . . 88

13.2.3 Unknown state . . . . . . . . . . . . . . . . 89

13.2.4 Angular distribution of the photoelectrons . . . . . . 91

14 Results at VUV wavelength $\quad 95$

14.1 Experimental results . . . . . . . . . . . . . . . . 95

14.2 Additional experiments . . . . . . . . . . . . . . . . . . . . . . . . . . . . . . 97

14.2.1 UV residue . . . . . . . . . . . . . . . . . . 99

14.2.2 IR residue in the VUV beam . . . . . . . . . . . . 100

14.2.3 Polarization . . . . . . . . . . . . . . . . . 101

14.2.4 Change in VUV intensity . . . . . . . . . . . . . 101

14.3 Results and discussion . . . . . . . . . . . . . . . . . . . . . . . . . . . . . . . . . . . . . . .

14.3.1 Peaks at low TKER . . . . . . . . . . . . 105

14.3.2 Peaks with high TKER . . . . . . . . . . 117 
14.3.3 Conclusion . . . . . . . . . . . . . . . . . . . . . . 122

$14.3 .42 .16 \mathrm{eV}$ feature . . . . . . . . . . . . . 122

15 Results at UV+VUV wavelength 126

15.1 Experimental results . . . . . . . . . . . . . . . . 126

15.2 UV wavelength independent rings . . . . . . . . . . . 130

15.2.1 Excitation to a repulsive state . . . . . . . . . . 130

15.2.2 Dissociation into two neutral I atoms . . . . . . . . 132

$15.3 \mathrm{UV}$ wavelength dependent rings . . . . . . . . . . . . . . 139

15.3.1 Dissociation of an ionic bound state . . . . . . . . . 139

15.3.2 Excitation of an intermediate of the neutral . . . . . 140

15.3.3 Three body decay . . . . . . . . . . . . . . . . . 143

16 Ion Pair states 148

17 Summary and Outlook $\mathbf{1 5 5}$

17.1 Summary . . . . . . . . . . . . . . . 155

17.2 Outlook . . . . . . . . . . . . . 156

17.3 Slicing technique . . . . . . . . . . . . . . 157

17.3.1 Slicing techniques so far . . . . . . . . . . . 158

17.3.2 Slicing and Velocity Map Imaging . . . . . . . . . . 159 


\section{Chapter 1}

\section{Introduction to Photochemistry}

The first photoinitiated experiments consisted of cell experiments in which the gas or the gases under study were photolysed with light from a flash source like a sodium or mercury lamp. These first experiment were mainly concerned with the determination of the chemical nature of the fragments themselves or their reactive products. With the arrival of laser light sources and the invention of molecular beams, the big revolution in the field of molecular dynamics in gas phase occurred. Since then, many more ideas, like new detection schemes (probe), preparation of reactants in certain states (pump), or influencing the characteristics of the molecular beam by manipulating the expansion condition more details can be extracted from the experiments or made it possible to obtain larger quantities of details in shorter time. Recent innovations enabled the more or less direct measurements of 3D-distributions.

Ideally, to fully characterize a reaction, or a dissociation, often considered a half-reaction, not only the identity of the reactant or parent molecule and the product is needed but a complete characterization of its states. But even a full knowledge of the quantum states of reactant and products does not show the full picture. As well the angular distribution, orientation and alignment are of importance. Thus it is desirable to prepare or determine the initial state as detailed as possible.

The aim of the first part of this work was to build an $\mathrm{OH}$ radical source to realize the $\mathrm{OH}+\mathrm{D}_{2} \rightarrow \mathrm{D}+\mathrm{HOD}$ reaction. Considerable effort was put into testing different precursors and different beam types.

Hence these results are not so much motivated by gaining new insights into the photodissociation dynamics of the examined species which are quite well known, but examining which molecule and its preparation and which dissociation process are best suited as an OH-precursor. In this study, Laser Induced Fuorescence (LIF) and Rydberg tagging Time of Flight (RydbergTOF) was used to determine the desired quantities of the beam. 
In the second part, photodissociation dynamics of $\mathrm{I}_{2}$ in the VUV / UV regime were studied. Here, Velocity Map Imaging (VMI) was employed as a new method in this laboratory. These experiments give insight into the potential structure above the first ionization limit of $\mathrm{I}_{2}$, of which little is known compared to the vast amount of work concerning the iodine molecule at lower excitation energies which is available in literature. 


\section{Chapter 2}

\section{Photodissociation and reaction dynamics}

In the following chapter the principles governing photodissocitaion and reactive collisions are outlined. These principles will be needed in both parts of this work. Part I studies the feasibility of realizing the $\mathrm{OH}+\mathrm{D}_{2}$ reaction using an $\mathrm{OH}$ beam which is produced by the photolysis of a suitable precursor. Part II studies the dissociation dynamics of $\mathrm{I}_{2}$ above the ionization threshold.

\subsection{Photodissociation and reactive collisions}

Photodissociation and reactive collisions are in general governed by the same physical laws. Photodissociation is usually considered a half collision.

To understand this, let us first consider the geometry and symmetry of a reactive collision. This is easiest when considering a Newton diagram. Conservation of energy and linear momentum gives the following equations within the centre-of-mass $(\mathrm{cm})$ system of the reaction $\mathrm{A}+\mathrm{BC} \rightarrow \mathrm{AB}+\mathrm{C} / \mathrm{C}^{*}$.

$$
\begin{array}{r}
E_{k i n}(A)+E_{\text {int }}(A)+E_{k i n}(B C)+E_{\text {int }}(B C)= \\
E_{k i n}(A B)+E_{k i n}(C)+E_{\text {int }}(A B)+E_{\text {int }}+\Delta D_{0}^{0}(B C-A B) \\
\text { with } \Delta D_{0}^{0}(B C-D C): \text { difference between BC and } \\
\text { AC in the dissociation energy } \\
\vec{P}=0=m_{A} \vec{u}_{A}+m_{B C} \vec{u}_{B C}=m_{A V} \vec{u}_{A B}+m_{B} \vec{u}_{B}
\end{array}
$$

In the laboratory frame the velocity vectors $v_{A}$ and $v_{B C}$ cross at right angles, see figure 2.1. The relative velocity and the centre-of-mass velocity is given by 


$$
\begin{array}{r}
v_{A}-v_{B C}=v_{r e l} \\
\vec{v}_{c m}=\frac{m_{A} \vec{v}_{A}+m_{B C} \vec{v}_{B C}}{m_{A}+m_{B C}} .
\end{array}
$$

With these equations we can change entirely to the centre-of-mass system (cm-system). The velocities within this system, $u_{A}$ and $u_{B C}$ are then directed along the relative velocity $\mathrm{v}_{r e l}$ inversely proportional to their masses $u_{A} / u_{B C}=m_{B C} / m_{A}$. The velocities of the reaction products can be similarly described, they are directed along a relative velocity $v_{r e l}^{\prime}$ with $u_{A B} / u_{C}=$ $m_{C} / m_{A B}$. One of the determining characteristics of a reactive collision is the scattering angle or ejection angle, which is defined as the angle between $v_{r e l}$ and $v_{r e l}^{\prime}$. If the scattering process has a preferred direction, anisotropic scattering is the consequence. But the ejection direction will always be cylindrically symmetric around the initial symmetry axis of the reaction, which is the initial relative velocity. In a Newton diagram, additionally to the velocities, concentric circles are given of which the radii represent the speed of the reaction products $u_{C}$ or $u_{C}^{*}$, see fig. 2.1. If we consider now only the cmsystem, the relative velocity $v_{r e l}$ gives the symmetry axis, and the velocities of the reaction products form cones around this axis.
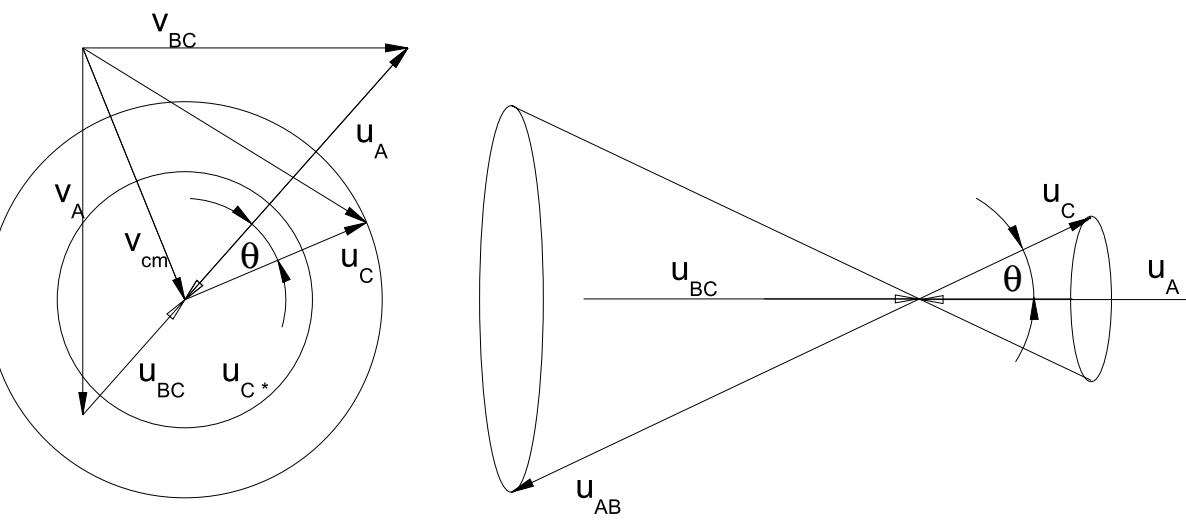

Figure 2.1: The newton sphere in the laboratory frame and its representation in the $\mathrm{cm}$-frame. $\theta$ is the $\mathrm{cm}$-frame scattering angle.

Now we come back to our initial problem, the photodissociation and its representation as a half collision.

Consider the photodissociation process

$$
A B+h \nu \rightarrow(A B)^{*} \rightarrow A+B / B^{*}
$$


where the complex is electronically excited in a parallel transition and followed by direct axial dissociation with two possible exit channels B and $\mathrm{B}^{*}$.

The centre of mass velocity is given by the thermal motion or the velocity obtained in the expansion of the molecular beam. The thermal motion will be small compared to the velocity gained in the dissociation process, and the velocity from the beam expansion is aimed in direction of the detector. Later we will see that the Newton sphere is compressed along this axis in the Velocity Map Imaging method and that the final velocity in this direction is dominated by the velocity gained in the acceleration process.

The fragments of the dissociation process move again in expanding spheres. The photodissociation process can be described as well with a Newton diagram, where the circles now represent the speed gained in the dissociation process. The axis of symmetry is here the polarization of the incident light. The angular distribution is now given with respect to this symmetry axis and depends solely on the photoexcitation step.

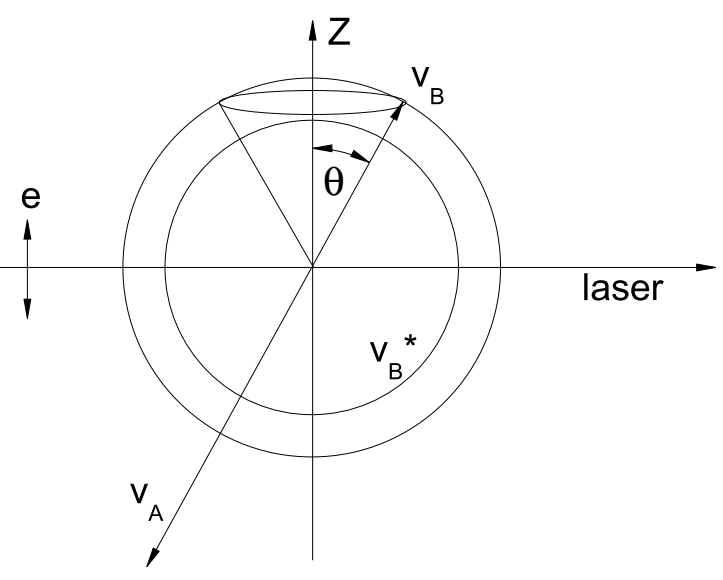

Figure 2.2: Newton sphere representing the photodissociation process.

The probability for a single photon absorption in an electric dipole transition is

$$
P_{a b s} \propto \cos ^{2} \theta,
$$

where $\theta$ denotes the angles between dipole transition moment $\vec{\mu}$ of the molecule and the electric vector $\vec{e}$ of the incident laser. Now there exist two possibilities. Either lies the transition moment parallel to the molecular axis, then the recoil velocity vector lies parallel to $\vec{\mu}$ and shows the same $\cos ^{2} \theta$ 
dependency with respect to $\vec{e}$, if it is perpendicular to $\vec{\mu}$, the recoil velocity vector distribution will exhibit a $\sin ^{2} \theta$ distribution with respect to $\vec{e}$.

\section{$2.2 \quad$ Photodissociation kinetics}

The speed distribution of the photofragments can be again easily obtained from the conservation of energy. The total kinetic energy (TKER) is given by the sum of the kinetic energy (KER) of each of the fragments by the equation below, with $\sum^{m} n_{m} h \nu_{m}$ the total photon energy, $E_{\text {int }}$ the appropriate internal energy, in case of an atomic fragment this can only be electronic excitation, and $D_{0}$ the dissociation energy:

$$
T K E R=K E R_{A}+K E R_{B}=\sum^{m} n_{m} h \nu_{m}-D_{0}(A B)-E_{\text {int }}(A)-E_{\text {int }}(B) .
$$

The initial cm-velocity of the parent molecule can be neglected, because the molecular beam points in direction of the detector. Since from velocities gained in the dissociation the components perpendicular to the molecular beam are measured (see chapter 11.1), the beam velocity does not contribute. Only the perpendicular spread of the beam will smear out the final velocities, but because the spread can add velocity components parallel and antiparallel to the measured velocities, the velocity from the dissociation process will be preserved as the central velocity of the smeared out distribution. From the FWHM of the final velocities a maximum velocity component from the beam of $\Delta v_{\text {beam }, \perp} \approx \pm 30 \mathrm{~m} / \mathrm{s}$ was determined. Since the initial cm-velocity can be neglected, we obtain from the conservation of linear momentum:

$$
m_{A} v_{A}+m_{B} v_{B}=0 .
$$

This gives then altogether the simple speed and kinetic energy ratios:

$$
\frac{v_{A}}{v_{B}}=\frac{m_{B}}{m_{A}}=\frac{K E R_{A}}{K E R_{B}} .
$$

Thus the TKER is fractioned into

$$
\begin{aligned}
K E R_{A} & =\frac{m_{B}}{m_{A}+m_{B}} \cdot T K E R \\
K E R_{B} & =\frac{m_{A}}{m_{A}+m_{B}} \cdot T K E R .
\end{aligned}
$$


If only the speed of one fragment is measured, the TKER can be extracted by

$$
T K E R=\frac{m_{A}+m_{B}}{m_{B}} \cdot K E R_{A} .
$$

In the case of the homonuclear diatomic, this reduces to a convenient $T K E R=2 \cdot K E R$.

The relation can also be expressed in terms of the reduced mass $\mu=$ $\left(m_{A} \cdot m_{B}\right) /\left(m_{A}+m_{B}\right)$ and the relative velocity of the fragments $v_{r e l}=v_{A}-v_{B}$ :

$$
T K E R=\frac{1}{2} \mu v^{2} .
$$

From the study of the fragment speed distributions information on the internal excitation can be gained, provided the bond dissociation energy $D_{0}$ is known. Vice versa, the dissociation energy can be determined, if the internal excitation is known because either state selective detection was used or because only a restricted number of asymptotes can be reached with the used photon energy which makes thus an assignment possible.

In the experiments performed in this work, it will later be seen (chapters 14 and 15) that not always straight forward dissociation is the case here. The analysis of the result shows that mostly dissociative ionization occurred. Concerning the fragments and their final relation to each other, i.e. the mass ratio, the same applies as in a neutral photodissociation process, but in the energy conservation, the kinetic energy of the electron or the term value of the intermediate dissociative state must be considered.

These exceptional cases are further explained and quantified when appropriate.

\subsection{Angular distribution of the fragments}

The angular distribution of the fragmentation products can give vital information about the photoexcitation step. As was mentioned before, the transition dipole moment $\mu$ lies preferentially along the electric vector of the incident light. With a linearly polarized light source, a highly anisotropic angular distribution of the fragments is the case. If $\mu$ lies along the internuclear axis, mostly molecules are excited which are oriented parallel to the 
electric vector of the light and ejection of the fragments parallel to it occurs mostly. If the life time is sufficiently short, i.e. much shorter than the rotational period of the excited transition state, the fragments will show a $\cos ^{2} \theta$ distribution. For a parallel transition is $\Delta \Omega=0$, these are transitions of the kind $\Sigma-\Sigma, \Pi-\Pi$, etc. A perpendicular transition with $\Delta \Omega= \pm 1$ which is e.g. $\Sigma-\Pi$ or $\Pi-\Delta$ (Hund's case A and B description) would yield a $\sin ^{2} \theta$ distribution, where the ejected fragments recoil perpendicular to the polarization of the light.

The general expression of the angular distribution from photofragmentation in a one-photon excitation step is $[1,2,3]$

$$
I(\theta) \propto \frac{1}{4 \pi}\left[1+\beta P_{2}(\cos \theta)\right]
$$

where $I$ is the intensity, $\beta$ the anisotropy parameter and $P_{2}(x)=\frac{1}{2}\left[3 x^{2}-1\right]$ the second order Legendre polynom and $\theta$ the angle between the electric vector $e$ of the laser light and the recoil velocity vector $v$. Normally the fragments leave with a high kinetic energy and the recoil direction is parallel to broken bond (axial recoil). But if the excitation energy is just above the threshold to dissociation and the fragments receive only very little kinetic energy from the dissociation compared to the rotational energy of the molecule, they are ejected with a component perpendicular to the bond axis by the rotational motion of the molecule (transverse recoil).

To give the anisotropy parameter $\beta$ is usually sufficient to describe the angular distribution. It ranges between -1 and $+2, \beta$ is -1 for a perpendicular transition which gives $I(\theta) \propto \sin ^{2} \theta$, and it is +2 for a parallel transition which gives $I(\theta) \propto \cos ^{2} \theta$. For $\beta=0$, the distribution is isotropic. The anisotropy parameter is

$$
\beta=2 P_{2}(\cos \chi)
$$

with $\chi$ the angle between the transition moment $\mu$ and the recoil velocity $v$.

The extreme values of $\beta$ can only be obtained if the photodissociation process takes place fast, before the rotation of the molecule destroys the anisotropy (direct dissociation), i.e. the dissociation lifetime $\tau$ of the excited intermediate must be smaller than its rotational period $\tau_{\text {rot }}$. If this does not hold true, the angular distribution information gets lost. The dependency of the anisotropy parameter on the dissociation lifetime and the rotational period is given by [4] 


$$
\beta(\tau)=2 P_{2}(\cos \chi) \cdot \frac{\omega^{2} \tau^{2}+1}{4 \omega^{2} \tau^{2}+1}
$$

with $\omega$ the angular velocity of the molecule, which gives thus the average rotation angle with $\omega \tau$. But even for extremely slow dissociation processes with $\tau \gg \tau_{\text {rot }}, \beta$ is only lowered by a factor 4 .

Not always are the extreme $\beta$ values reached even in a direct dissociation. It may be that the transition moment is not exactly parallel or perpendicular to the molecular axis, as well it is possible that the transition has a mixed character with both parallel and perpendicular contributions because the excited state may not be a pure state but has contributions from different electron configurations.

The general form of the angular distribution is valid for the one photon absorption. For a multiphoton absorption the angular distribution can be given by a longer Legendre expansion. For a two photon process this is

$$
I(\theta) \propto \frac{1}{4 \pi}\left[1+\beta P_{2}(\cos \theta)+\gamma P_{4}(\cos \theta)\right] .
$$

In the case that an intermediate state dominates the first absorption step, this will change because of the strong determining influence. In that case, the absorption must be regarded stepwise. Without a dominating state, all intermediate states do contribute, they are weighted according to their oscillator strength and proximity to virtual one-photon level in the excitation process.

\section{Bibliography}

[1] R.N. Zare and D.R. Hershbach. Proc. IEEE, 51:173, 1963.

[2] R.N. Zare. Mol. Photochem., 4:1, 1972.

[3] R.N. Zare. Angular Momentum, Understanding spatial Aspects in Chemistry and Physics. John Wiley and Sons, Inc., New York, 1988.

[4] C. Jonah. Jour. Chem. Phys., 55:1915, 1971. 


\section{Chapter 3}

\section{Experimental Set-up}

The experimental set-up was designed to study photodissociation and reaction dynamics of molecules in a molecular beam set-up.

In this chapter only the part of the experimental set-up is described which applies to both parts of this work. Alterations which only apply to specific experiments will be described in separate chapters when needed.

The whole experimental set-up consists of the vacuum chamber with the pumps, the laser systems, the gas systems with the pulsed valves or the effusive source and the equipment for controlling and data sampling.

The apparatus itself allows for two parallel molecular beams which can be separately dissociated or probed. In plane of the upper beam, a VUV laser beam which needs a special set-up (see section 3.2.1) is available and crosses the molecular beam at a right angle. Also a second laser beam can be directed into the chamber via a second pair of windows. This axis is rotated by 4 degree with respect to the VUV axis. In plane of the lower beam, two laser beams can be counterpropagated on an axis perpendicular to the molecular beam. Various detectors can be used, including a secondary electron multiplier which is rotatable around the VUV axis and a position sensitive multi channel plate detector opposite of the upper beam. These configurations allow angular resolved measurements of either reaction or dissociation products.

\subsection{The vacuum apparatus}

The high vacuum chamber is a cylinder made of stainless steel with $\mathrm{CF}$ and ISO-K flanges and has a diameter of $800 \mathrm{~mm}$ and a height of $730 \mathrm{~mm}$. It is divided into a main chamber and a differentially pumped one which can be divided into two, where each chamber can hold a molecular beam valve. 
For the main volume two pumps are available, a cryopump (manufacturer.: Leybold-Hereaus, type: RPK 3500 with compressing unit RW2, pump rate: $\left.4500 \mathrm{l} / \mathrm{s}\left(\mathrm{H}_{2}\right)\right)$ and a turbo molecular pump, corrosive gas safe make (manu.: Pfeiffer-Balzers, type: TPU $510 \mathrm{~S}$ pump rate: $480 \mathrm{l} / \mathrm{s}\left(\mathrm{H}_{2}\right)$ ). A dualstage rotary vane pump (manu.: Leybold-Hereaus, type: Triviac B D16B) with adsorption trap (manu.: Leybold-Hereaus, type: adsorption trap NW 25 ) is used as a backing pump. During times the apparatus is not used, the turbo pump is kept working to maintain the vacuum at about $2 \cdot 10^{-7}$ mbar. With all pumps working and without gas load, pressure was down to approximately $1 \cdot 10^{-9} \mathrm{mbar}$ and with gas load about $4 \cdot 10^{-8} \mathrm{mbar}$. For the reaction chamber, a partial pressure measurement unit (manu.: Edwards, type: VG Arga) was used.

The differentially pumped source chamber is pumped by a cryopump (manu.: Leybold-Hereaus, type: RPK 1500, pump rate: 2000l/s $\left(\mathrm{H}_{2}\right)$ with compressor unit RW2 ) and an oil diffusion pump with a liquid nitrogen cooled baffle (manu.: Edwards, type: Diffstak CR 250 pump rate: 3500l/s $\left(\mathrm{N}_{2}\right)$ ) with a dual-stage rotary vane pump (manu.: Leybold-Hereaus, type: Trivac D $40 \mathrm{~B}$, pump rate: $40 \mathrm{~m}^{3} / \mathrm{h}$ ) as a backing pump. The pressure is measured by an ionization gauge (manu.: Leybold-Hereaus, type: IE 413 with display: Ionivac IM 510). Without gas load, pressure was about $2 \cdot 10^{-6}$ mbar.

When divided into two differentially pumped chambers, the cryopump pumps the upper volume, the oil diffusion pump the lower volume. The pressure in the lower chamber is as well measured by an separate ionization gauge (manu: Leybold-Hereaus, type: IE 411, display: Combivac IT 230).

\subsection{The laser systems}

A variety of laser systems were available which allowed the production of a wide range of wavelength:

- ArF Excimer Laser, manu.: Lambda Physics, type: EMG 101, pulse width $10 \mathrm{~ns}$, energy /pulse 40-100 mJ at $193 \mathrm{~nm}$

- Nd:YAG Laser, manu.: Spectra Physics (Quanta Ray), type: DCR 1A, with a donut profile. The fundamental of $1.064 \mu \mathrm{m}$ has a bandwidth of $1 \mathrm{~cm}^{-1}$, and a pulse length of $8 \mathrm{~ns}$. The frequency was doubled or quadrupled by one or two KD*P crystals to obtain either $532 \mathrm{~nm}$ with $\approx 120 \mathrm{~mJ}$ per pulse which was used as a dyelaser pump $(308 \mathrm{~nm})$ or $266 \mathrm{~nm}$ with $4 \mathrm{~cm}^{-1}$ bandwidth, a pulse length of $5 \mathrm{~ns}$, and an energy of $20-30 \mathrm{~mJ}$ per pulse. The polarization of the $266 \mathrm{~nm}$ radiation can be 
rotated by a half wave plate. It was focused into the chamber with a quartz lens of $1000 \mathrm{~mm}$ focal length.

- Nd:YAG Laser, manu.: Spectra Physics (Quanta Ray), type GCR 5 with injection seeder. The second and third harmonics, $532 \mathrm{~nm}$ and $355 \mathrm{~nm}$ respectively were used to pump the two dye lasers necessary for the VUV and the $365 \mathrm{~nm}$ wavelength production.

- two dye lasers (manu: Spectra Physics, type: PDL-1), one pumped with $355 \mathrm{~nm}(115 \mathrm{~mJ} /$ pulse) giving $424 \mathrm{~nm}(15 \mathrm{~mJ} /$ pulse, Bis-MSB in dioxane), doubled to $212.55 \mathrm{~nm}(1 \mathrm{~mJ})$ and one pumped with $532 \mathrm{~nm}$ (95 J/pulse) giving $\approx 845 \mathrm{~nm}(200 \mu \mathrm{J} /$ pulse, oscillator only; Styril 9 in DMSO)

- two dye lasers (manu.: Lambda Physics, type: FL 2002), both $532 \mathrm{~nm}$ (100 mJ/pulse) pumped giving around $620 \mathrm{~nm}$ (Rhodamine 101 in methanol), doubled to $310 \mathrm{~nm}(5 \mathrm{~mJ} /$ pulse) and $730 \mathrm{~nm}$ (Pyridine 2 in methanol) doubled to $365 \mathrm{~nm}(3-8 \mathrm{~mJ} /$ pulse $)$

The resulting wavelengths were then used for different experiments:

- $266 \mathrm{~nm}$, dissociation of $\mathrm{HI}$ (calibration of $\mathrm{I}_{2}$ dissociation images) and used in the UV+VUV dissociation measurements of $\mathrm{I}_{2}$

- $212.55 \mathrm{~nm}$, production of VUV and, since after the VUV production there is still some $212.55 \mathrm{~nm}$ laser light remaining in the beam , also photodissociation and ionization of $\mathrm{I}_{2}$

- $121.53 \mathrm{~nm},($ Lyman- $\alpha(\mathrm{D}))$, ionization and dissociation measurements of $\mathrm{I}_{2}$. Also used for the first step in Rydberg tagging TOF or 1+1-REMPI of hydrogen.

- $193 \mathrm{~nm}$, photodissociation of $\mathrm{H}_{2} \mathrm{O}_{2}$ and $\mathrm{NHO}_{3}$

- $\approx 365 \mathrm{~nm}$, used in the VUV+UV dissociation measurements of $\mathrm{I}_{2}$, also the second step in Rydberg tagging or 1+1-REMPI

- 308-312 nm, LIF wavelength of $\mathrm{OH}$.

To control the power of the various lasers, a variety of different pyroelectric detectors were used: type PEM25 with $9.8 \mathrm{mV} / \mathrm{mJ}$ and type PEM34 with $50 \mathrm{mV} / \mathrm{mJ}$ (manu: Radiant Dye Laser Acc.), type ED-500 with 2.5mV/mJ (manu: gentec) and type $3 \mathrm{eA}-\mathrm{P}$ with $0.349 \mathrm{mV} / \mathrm{W}$ (manu: ophir). 


\subsubsection{The VUV laser}

The VUV production of $121.57 \mathrm{~nm} / 121.53 \mathrm{~nm}$ is a non-linear optical process with an experimental technique which is rather complex and laborious to maintain. But since the technique is well established because it was needed to excite $\mathrm{H}$ - or D-atoms from the 1 s state to the $2 \mathrm{p}$ state (Lyman- $\alpha$ radiation) when using Rydberg tagging time-of-flight in previous experiments, the way of producing VUV was kept.

It is produced by using resonant four-wave mixing (difference frequency mixing) in a non-linear optic medium. For an isotropic medium, the efficiency of this process depends on the third order susceptibility $\chi_{3}$ only (second order is zero, higher orders are negligible).

For this process eq. 3.1 is valid, which represents energy conservation:

$$
\omega_{4}=\omega_{1}+\omega_{2}-\omega_{3}
$$

with $\omega_{i} \quad$ : for $\mathrm{i}=1-3$ initial, $\mathrm{i}=4$ product frequency

The third order susceptibility is largest when a resonant transition of the non-linear medium is involved. Because the transition probability of a twophotons process is much smaller than for a one-photon process, absorption losses are considerably smaller, and it is reasonable to use two photons to match the resonant transition wavelength.

Here, as the non-linear optic medium, krypton is used, the resonant state is the $4 p^{5} 5 \mathrm{p}[1 / 2,0]$ level. Two UV photons of $212.55 \mathrm{~nm}$ are used to match the resonant step, and one infrared photon of $846 \mathrm{~nm}$ is subtracted to obtain the needed $121.53 \mathrm{~nm}$.

$$
\omega_{V U V}(121.53 \mathrm{~nm})=2 * \omega_{U V}(212.55 \mathrm{~nm})-\omega_{I R}(846 \mathrm{~nm})
$$

For a collinear geometry holds:

$$
\begin{gathered}
\Delta k=k_{V U V}-\left(2 k_{U V}-k_{I R}\right)=0 \\
\text { with } \mathrm{k} \quad: \text { wavevector }
\end{gathered}
$$

To satisfy eq. 3.3, the phase velocities, which means the dispersion coefficients of the pumping and resulting wavelength have to be adjusted to each other. This could be done by choosing the pressure of the krypton accordingly, because the VUV and UV wavelength are divided by a region of 
abnormal dispersion, but the efficiency can be greater when a mixture of a gas with negative and one with positive dispersion is used. A mixture of argon and krypton can be used for the wavelength in interest, because krypton has a negative dispersion up to $123.6 \mathrm{~nm}$ and argon is positively dispersing in the region from $121.1 \mathrm{~nm}$ to $180 \mathrm{~nm}$.

The relation of the mixture depends on the needed wavelength, here it was 1:3.4 krypton to argon.

The two laser beams are collinearly focused with a quartz lens of $550 \mathrm{~mm}$ (at $212 \mathrm{~nm}$ ) focal length into the VUV cell, which contains the argon-krypton mixture. Both gases are fed through a cooled tubing system to remove freezable impurity gases like water and improve mixing conditions.

Before the two laser beams are combined using a special dielectric mirror (highly reflecting for $212 \mathrm{~nm}$, highly transmitting at $843 \mathrm{~nm}$ ), a telescope lens system is inserted into the beam path of the IR laser to make up for the difference in focal length of the quartz lens for the two different wavelengths. The resulting beam is then introduced from the VUV cell into the reaction chamber by a plano-convex $\mathrm{MgF}_{2}$ lens with $\mathrm{f}=200 \mathrm{~mm}$ at $121 \mathrm{~nm}$. Behind the reaction chamber, it enters an ionization cell which contains $1 \% \mathrm{NO}$ in Helium, where the Lyman- $\alpha$ photons are detected by ionizing NO. Pressure in the NO cell was usually about $0.5 \mathrm{mbar}$. The ions were withdrawn by a voltage of $120 \mathrm{~V}$. The ion signal was calibrated to obtain the photons per pulse [1]. A typical pulse had about $10^{12}$ photons. This gives an areal density of $1.3 \cdot 10^{14}$ photons $/ \mathrm{cm}^{2}$ of $121.53 \mathrm{~nm}$ photons in the probe volume with a pulse length of $5 \mathrm{~ns}$, which is enough to saturate the first D atom detection step, see chapter 5.2.

The process of VUV generation is described in detail in ref. [2].

\subsection{Delay control}

For running the experiment, it is essential to adjust laser/valve trigger times to each other.

To do so, the start time of one component of the experiment was kept fixed. The time of all other components were then set relative to it. Here, as the fixed zero of the timescale, the Q-switch time of the Nd:YAG which pumps the probe lasers was used.

To control the components, one delay unit with five separate outputs (manu: electronic workshop, University of Bielefeld) and a couple of delay units (manu: Ortec, type: Gate\&Delay 416A) were used. 
Repetition rate of the experiment is $10 \mathrm{~Hz}$, given by the maximum repetition rate of the Nd:YAG lasers.

\section{Bibliography}

[1] W. Meier. Zustandsselektive Laserspektroskopie an molekularem Wasserstoff. Dissertation, Universität Bielefeld, Fakultät für Physik, Oktober 1986.

[2] J. Borkowski. Erzeugung intensiver abstimmbarer gepulster Laserstrahlung im $V U V$. Diplomarbeit, Universität Bielefeld, Fakultät für Physik, June 1993. 
Part I

\section{OH-beam}




\section{Chapter 4}

\section{Introduction to the $\mathrm{OH}-$ Source}

The dynamics of molecular reactions can be determined in molecular beam experiments as well as in cell experiments. The advantage of molecular beam experiments is that the initial states and velocities are better defined and can be influenced, which will yield better defined results, whereas cell experiment give results averaged over a thermal distribution of the initial states. But in both cases it is often necessary to prepare the reactant in a way that the total energy is sufficient to overcome a potential barrier to the reaction.

The $\mathrm{OH}+\mathrm{H}_{2} \longrightarrow \mathrm{H}_{2} \mathrm{O}+\mathrm{H}$ reaction is the benchmark system for 4-atom reaction because it is the simplest non-trivial 4-atomic system which can be treated fully quantum mechanically, as was the hydrogen exchange reaction $\mathrm{H}+\mathrm{H}_{2} \longrightarrow \mathrm{H}_{2}+\mathrm{H}$ the benchmark system for the 3-atom reaction. Experimentally, the realization is quite challenging because it has a high barrier to it, which is $0.174 \mathrm{eV}\left(4.0 \mathrm{kcal} / \mathrm{mol}\right.$ ) for $\mathrm{OH}+\mathrm{H}_{2} \longrightarrow \mathrm{H}_{2} \mathrm{O}+\mathrm{H}$ and $0.230 \mathrm{eV}$ $(5.3 \mathrm{kcal} / \mathrm{mol})$ for its isotopic variant $\mathrm{OH}+\mathrm{D}_{2} \longrightarrow \mathrm{HOD}+\mathrm{H}$.

Experimentally, much research was undertaken on behalf of the reaction $[1,2,3,4,5,6,7,8,9,10,11,12,13]$, giving rate coefficients for both the $\mathrm{OH}+\mathrm{H}_{2}$ reaction as for its reverse, the $\mathrm{H}+\mathrm{H}_{2} \mathrm{O}$ reaction, among the more recent works some are treating the weakly bound complex $\mathrm{OH}-\mathrm{H}_{2}$ at the entrance of the reaction channel [14, 15, 16, 17, 18]. A recent overview article was published by Smith and Crim [19], detailing experimental and theoretical progress on the $\mathrm{OH}+\mathrm{H}_{2}$ reaction, its reverse reaction and its isotopic variants.

First experimental measurements of the system in a crossed beam set-up were performed in the group of Casavecchia in Perugia [12], although with a low resolution, but still with enough detail that the preference for high vibrational modes could be discovered.

Up to then, although the system was studied in experiments many times, 
very few experimental results existed for this fundamental reaction which gave such details. Thus very little is known about its exact quantum distribution or even steric qualities, e.g. the planarity of the reaction.

On the other hand, this reaction can be treated theoretically in fully converged 6-dimensional quantum calculations [20, 21, 22, 23, 24, 25]. State resolved differential cross sections are available [26, 27], which were calculated on the potential energy surface by Ochoa de Aspuru and Clary [28], as well as reaction rates $[29,30]$. Newer integral cross sections were calculated by Yang et al. [31, 32, 33]. These calculations were performed on the latest PES by Bettens et al. [34], its better, second version [35] and its extension [36]. Thus the $\mathrm{OH}+\mathrm{H}_{2}$ system can be considered as the established benchmark system, where the still small number of electrons is its advantage. Some problems have been tackled within the last years with quasiclassical trajectory calculations, for example the occurrence and influence of a geometric phase effect in this reaction [37].

As quasiclassical trajectory calculations do not give the required amount of detail and exactness for a real prediction of experimental behaviour, do experimental results summed up or averaged over many states not give the depth needed to evaluate a theoretical model. Thus angular resolved state selective differential cross sections are needed, as they were determined in Bielefeld for the hydrogen exchange reaction [38, 39].

To experimentally realize a reaction with a barrier, in general the best way to overcome the barrier is to provide the sufficient energy as translational energy. For the $\mathrm{OH}+\mathrm{H}_{2} \longrightarrow \mathrm{H}_{2} \mathrm{O}+\mathrm{H}$ reaction it is known that the vibrational excitation of the $\mathrm{H}_{2}$ bond is helpful in promoting the reaction. Light and Matsumoto [40] found that the reaction rate was enhanced by a factor of ca. 150 for $\mathrm{H}_{2}(\mathrm{v}=1)$, but a similar vibrational excitation of the $\mathrm{OH}$ bond only gives an enhancement of $50 \%$. But even then is the thermally averaged reaction cross section for $\mathrm{OH}+\mathrm{H}_{2}(\mathrm{v}=1)$ only around $0.05 \cdot 10^{-16} \mathrm{~cm}^{2}$, although $52.1 \mathrm{~kJ} / \mathrm{mol}$ was provided as vibrational energy, whereas for a translational energy of $21.4 \mathrm{~kJ} / \mathrm{mol}$ in a collision a reaction cross section of $0.60 \cdot 10^{-16} \mathrm{~cm}^{2}$ was determined [6].

Thus it is also for this reaction reasonable to supply the energy needed to overcome the barrier as translational energy.

The idea was to use Rydberg tagging time-of-flight, which was developed in Bielefeld, on the isotopic variant $\mathrm{OH}+\mathrm{D}_{2} \rightarrow \mathrm{HOD}+\mathrm{D}$ and examine the reaction in the same fashion as the hydrogen exchange reaction. The reactively produced D atoms are excited in a two photon scheme (see chapter 


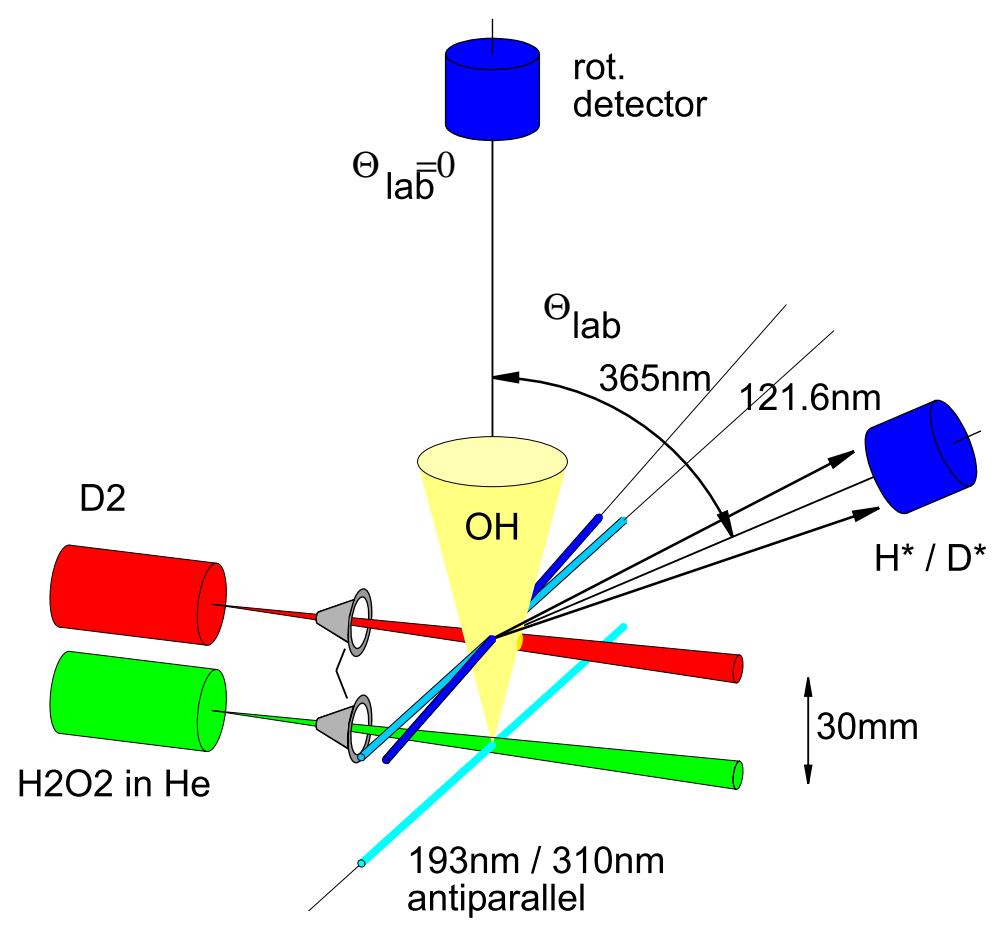

Figure 4.1: The principle of a crossed beam reaction realization. The $D_{2}$ beam and the precursor beam run parallel. The precursor is dissociated with a laser perpendicular to the beam. The $\mathrm{OH}$ radicals then expand in a spherical shell, here indicated by the cone expanding into the $D_{2}$ beam, and eventually meet the $\mathrm{D}_{2}$ beam. There the $\mathrm{OH}$ and the $\mathrm{D}_{2}$ react, and the formed $D$ atoms are probed with Rydberg tagging excitation scheme. The Rydberg $D$ atoms drift then to the detector, which can be rotated around the probe laser axis. Because the reaction has a symmetry axis given by the relative velocity which lies in the plane spanned by the molecular beams, the detector moves through the angle dependent distribution of the $D$ atoms along the symmetry axis.

5) to high Rydberg states which pass a drift path as neutrals to a rotatable detector where they are fieldionized directly in front of it (see fig. 4.1). From the energy distribution of the $\mathrm{D}$ atoms the vibrational quanta of the partner fragment HOD can be determined. This approach seemed feasible, since in the reaction the passive $\mathrm{OH}$ bond behaves as a spectator bond so that only vibrational excitation of two bonds of the product molecule contributed to the overall energy balance, the OD bond stretching mode and the HOD bending mode.

The principle of a crossed molecular beam set-up where one beam is 
produced by dissociation of a suitable precursor molecule is shown in fig. 4.1.

To provide a suitable beam of $\mathrm{OH}$ radicals for the $\mathrm{OH}+\mathrm{H}_{2} \longrightarrow \mathrm{H}_{2} \mathrm{O}+\mathrm{H}$ reaction, the $\mathrm{OH}$ properties and yields of different photodissociation processes which give $\mathrm{OH}$ radicals as fragments were investigated in this work. As mentioned above, it is necessary that the radicals have a high velocity $(>3500 \mathrm{~m} / \mathrm{s})$ and a narrow speed distribution. As well a peaked angular distribution is desirable to achieve a high density of $\mathrm{OH}$ radicals in the beam.

\section{Bibliography}

[1] F.P. Tully and A.R. Ravishankara. Jour. Phys. Chem., 84:3126, 1980.

[2] A.R. Ravishankara, J.N. Nicovitch, R.L. Thompson, and F.P. Tully. Jour. Phys. Chem., 85:2498, 1981.

[3] K. Kleinermanns and J. Wolfrum. Appl. Phys. B, 34:5, 1984.

[4] A. Jacobs, H.-R. Volpp, and J. Wolfrum. Jour. Chem. Phys., 100:1936, 1994.

[5] A. Jacobs and H.-R. Volpp J. Wolfrum. Chem. Phys. Lett., 196:249, 1992.

[6] S. Koppe, T. Laurent, P.D. Naik, H.-R. Volpp, and J. Wolfrum. Can. J. Chem., 72:615, 1994.

[7] R.A. Brownsword, M. Hillenkamp, T. Laurent, R.K. Vatsa, H.-R. Volpp, and H.-S. Yoo. Jour. Phys. Chem., 101:6448, 1997.

[8] R.A. Brownsword, T. Laurent, R.K. Vatsa, H.-R. Volpp, and J. Wolfrum. Chem. Phys. Lett., 259:375, 1996.

[9] K. Kessler and K. Kleinermanns. Chem. Phys. Lett., 190:145, 1992.

[10] K. Honda, M. Takayanagi, T. Nishiya, H. Ohoyama, and I. Hanazaki. Chem. Phys. Lett., 180:321, 1990.

[11] D. E. Adelman, H. Xu, and R. N. Zare. Chem. Phys. Lett., 203:573, 1993.

[12] M. Alagia, N.Balucani, P.Casavecchia, D. Stranges, G.G. Volpi, D.C. Clary, A. Kliesch, and H.J. Werner. Chem. Physics, 207:389, 1996. 
[13] M. Alagia, N. Balucani, P. Casavecchia, D. Stranges, and G.G. Volpi. J. Chem. Soc. Farad. Trans., 4:575, 1995.

[14] M.D. Wheeler, D.T. Anderson, M.W. Todd, M.I. Lester, P.J. Krause, and D.C. Clary. Mol. Phys., 97:151, 1999.

[15] D.T. Anderson, M.W. Todd, and M.I. Lester. Jour. Chem. Phys., 110:11117, 1999.

[16] M.D. Wheeler, M.W. Todd, M.I. Lester, and D.T. Anderson. Jour. Chem. Phys., 110:6732, 1999.

[17] J.M. Hossenlopp, D.T. Anderson, M.W. Todd, and M.I. Lester. Jour. Chem. Phys., 109:10707, 1998.

[18] R.A. Loomis and M.I. Lester. Jour. Chem. Phys., 103:4371, 1995.

[19] I.W.M. Smith and F.F. Crim. Phys. Cem. - Chem. Physics, 4:3543, 2002.

[20] J. Palma and J. Echave. Jour. Chem. Phys., 104:2841, 1996.

[21] D.H. Zhang, J.Z.H. Zhang, Y.Z.D. Wang, and Q. Zhang. Jour. Chem. Phys., 102:7400, 1995.

[22] D. Neuhauser. Jour. Chem. Phys., 100:9272, 1994.

[23] H. Szichman and M. Baer. Chem. Phys. Lett., 242:8620, 1995.

[24] D.C. Clary. Jour. Phys. Chem., 98:10678, 1994.

[25] W.H. Thompson and W. H. Miller. Jour. Chem. Phys., 101:8620, 1994.

[26] S.K. Pogrebnya, J. Palma, D.C. Clary, and J.Echave. Phys. Cem. Chem. Physics, 2:693, 2000.

[27] S.K. Pogrebnya, J. Echave, and D.C. Clary. Jour. Chem. Phys., 107:8975, 1997.

[28] G. Ochoa de Aspuru and D.C. Clary. Jour. Phys. Chem. A, 102:9361, 1998.

[29] F. Matzkies and U. Manthe. Jour. Chem. Phys., 108:4828, 1997.

[30] U. Manthe and F. Matzkies. Jour. Chem. Phys., 113:5725, 2000. 
[31] M. Yang, D.H. Zhang, M.A. Collins, and S.-Y. Lee. Jour. Chem. Phys., 114:4759, 2001.

[32] D.H. Zhang, M. Yang, and S.-Y. Lee. Jour. Chem. Phys., 114:8733, 2001.

[33] D.H Zhang, M. Yang, and S.-Y. Lee. Jour. Chem. Phys., 116:2388, 2002.

[34] R.P.A. Bettens, M.A. Collins, M.J.T. Jordon, and D.H. Zhang. Jour. Chem. Phys., 112:10162, 2000.

[35] D.H. Zhang, M.A. Collins, and S.-Y. Lee. Science, 290:961, 2000.

[36] M. Yang, D.H. Zhang, M.A. Collins, and S.-Y. Lee. Jour. Chem. Phys., 115:174, 2001.

[37] G. Billing and A. Kuppermann. Chem. Phys. Lett., 294:26, 1998.

[38] L. Schnieder, K. Seekamp-Rahn, J. Borkowski, K. H. Welge E. Wrede, F. J. Aoiz, L. Bañares, M. J. D’Mello, V. J. Herrero, V. Sáez-Rábanos, and R. E. Wyatt. Science, 269:207, 1995.

[39] L. Bañares, M.J. D’Mello, F.J. Aoiz, V.J. Herrero, B. Niederjohann, K. Seekamp-Rahn, E. Wrede, and L. Schnieder. Jour. Chem. Phys., 108:6160, 1998.

[40] G.C. Light and J.H. Matsumoto. Chem. Phys. Lett., 58:578, 1978. 


\section{Chapter 5}

\section{Experimental Set-Up for $\mathrm{OH}$ detection}

\subsection{LIF set-up}

During the experiments to study the variety of options for an $\mathrm{OH}$-source, two detection schemes were used. To directly probe the rotational distribution of the $\mathrm{OH}$-fragments from various dissociation processes, Laser Induced Fluorescence was used. The $\mathrm{OH}$ radicals were excited from the $\mathrm{X}^{2} \Pi$ state to the $\mathrm{A}^{2} \Sigma$ state in the wavelength range of $307-312 \mathrm{~nm}$, depending on the probed branch. The q-branch of the LIF transitions of $\mathrm{OH}$ lies between 308$312 \mathrm{~nm}$ wavelength, the most probable rotational excitation of $\mathrm{j}=10$ and 11 can be detected at approximately $310 \mathrm{~nm}$. The emitted LIF light is then collected with a suitable system and detected with a photomultiplier.

Since a system was wanted which could remain in the vacuum chamber during the reaction experiments, a small lens system consisting of two lenses with $12 \mathrm{~mm}$ focal length was introduced into the chamber. With this lens system, the light from the LIF processes was focused into a optical fused silica fibre. This fibre was approximately $1.5 \mathrm{~m}$ long and $1 \mathrm{~mm}$ in diameter and was introduced into the chamber via a vacuum feedthrough. To avoid mechanical strain, the feedthrough allowed a diameter of $5 \mathrm{~mm}$, and the sealing against vacuum was achieved with a solid rubberblock of approximately $5 \mathrm{~mm}$ in diameter with a small hole to allow the fibre instead of an o-ring seal.

The light from the fibre was then directed onto an UV sensible photomultiplier which was altered by the electronical workshop to allow gating. To block out ambient light, an UG11 filter was employed. To block out stray light from the laser, the gate of the PMT was set after the laser pulse of approximately $5 \mathrm{~ns}$, since the LIF signal is delayed in relation to the laser 
and has a lifetime of approximately $1 \mu \mathrm{s}$.

The signal from the PMT was monitored with an oscilloscope (manu: LeCroy, type: 9400A), the LIF spectra were recorded from the integrated readout of the oscilloscope with a $\mathrm{PC}$ via a GBIB connection, with the PC simultaneously controlling the LIF wavelength.

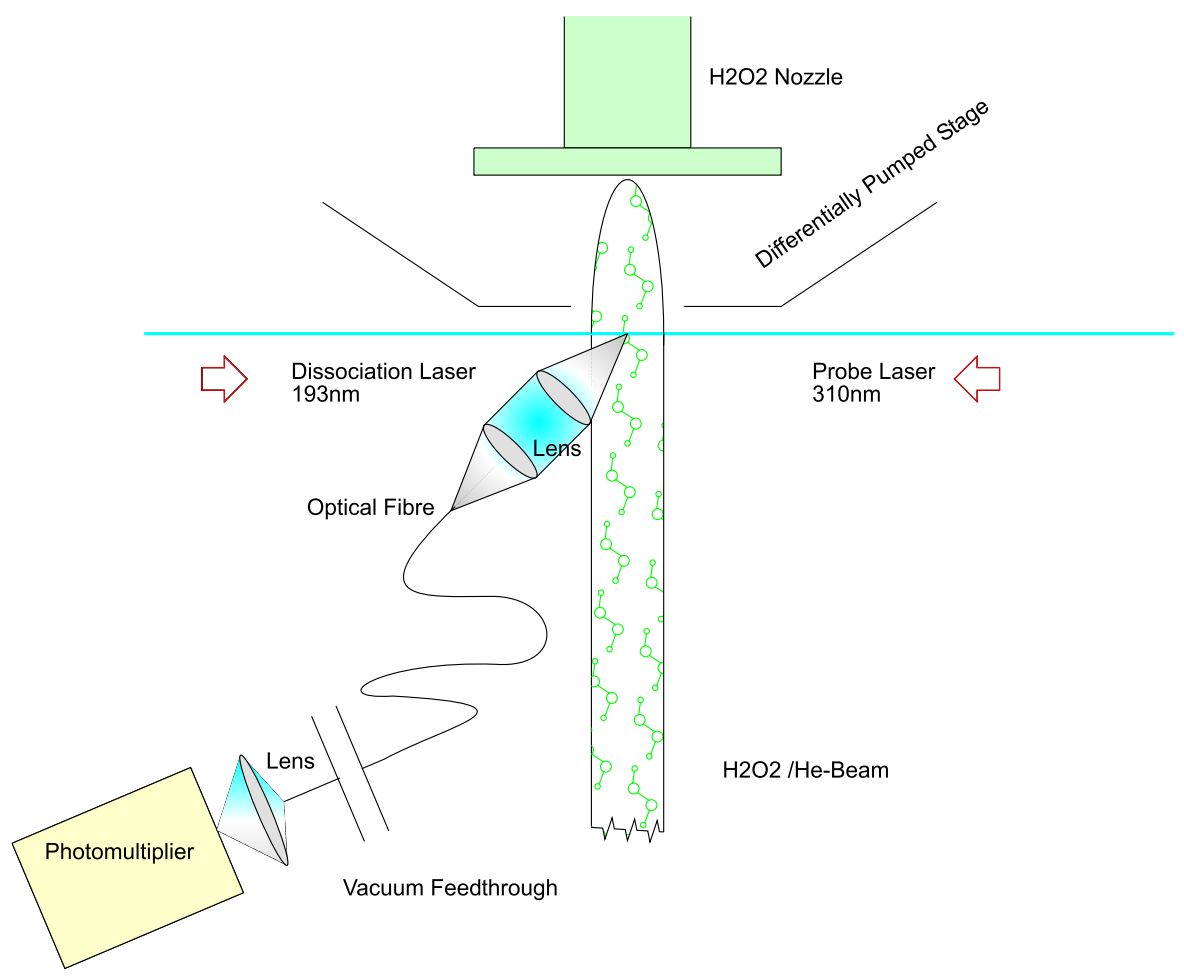

Figure 5.1: Schematic view of the lens position which couples the LIF light into the fibre optic.

The LIF lens system had to be adjusted in height prior to the experiments. To measure the LIF spectra it was adjusted to the height of the molecular beam with the $\mathrm{OH}$-precursor gas. This was done when the chamber was brought up to air. The pinholes which can be moved into the laser path (see sec. 5.2.1) were used to adjust the laser into position before the nozzle. Then a small sheet of paper was attached to the pinholes. Since the mask with the pinholes also intersects with the path of the molecular beams, the laser spot on the paper is now within the volume where LIF occurs during the experiments. The lens system was then adjusted for maximum signal using the scattered light from the laser spot on the paper. 


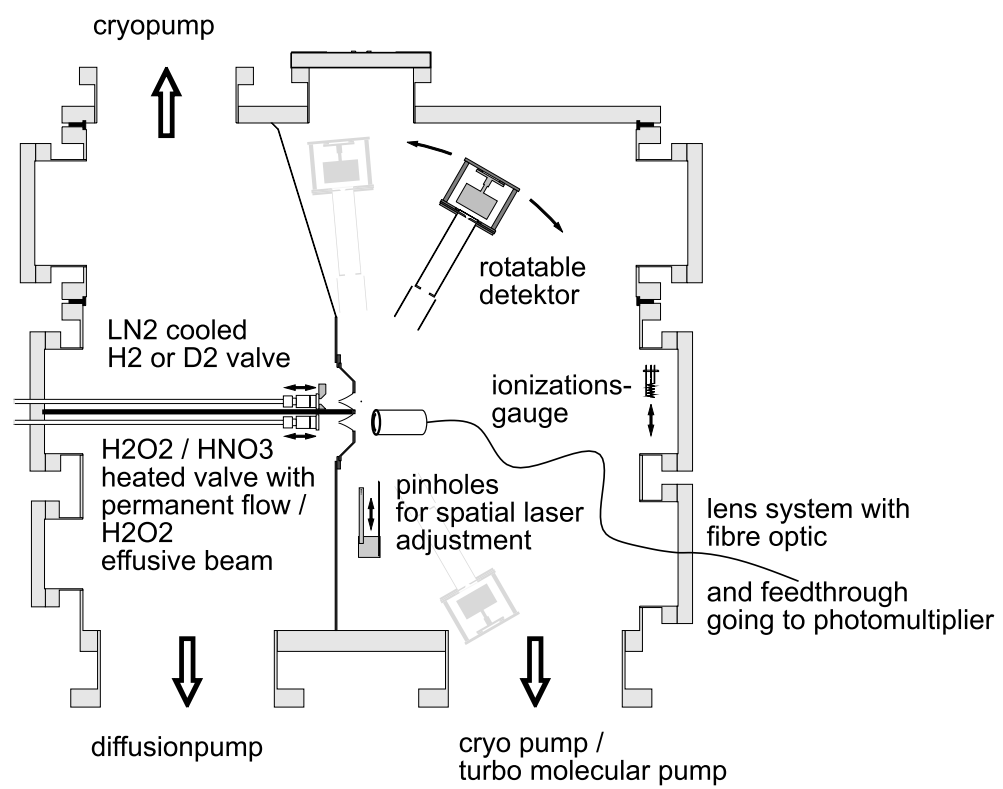

Figure 5.2: Vertical cut through the vacuum chamber, showing the position of the beam sources, the lens system, the rotatable detector and the movable mask

\subsection{Hydrogen detection}

To detect the Hydrogen atoms from either a reaction but also from dissociation experiments, the Rydberg tagging detection scheme was used. Hydrogen and deuterium atoms are excited to high metastable Rydberg states $(\approx 40)$ by resonant two photon excitation:

$$
\begin{array}{r}
H(1 s)+h \nu(121.57 n m) \longrightarrow H(2 p)+h \nu(365 n m) \longrightarrow H^{*}(n) \\
\text { or } \\
D(1 s)+h \nu(121.53 n m) \longrightarrow D(2 p)+h \nu(365 n m) \longrightarrow D^{*}(n)
\end{array}
$$

The Rydberg tagged $\mathrm{H}^{*}$ and $\mathrm{D}^{*}$ atoms then pass a drift path as neutrals to a secondary electron multiplier which is attached to a rotatable mount, which allows the angle dependent measurement of reactive products. In front of the detector surface, the Rydberg atoms are field ionized at a grid and then accelerated onto the detector. The detector surface was $5 \mathrm{~cm}^{2}$. A slit mask can be attached, with enhances angular resolution and was used during the hydrogen exchange reaction experiments, but reduces the active surface to $1.25 \mathrm{~cm}^{2}$. Since it was expected that the reaction experiments now would be low in signal, the mask was not used. 
For the dissociation experiments, the detector was kept fixed in a vertical position to the laser and molecular beams. To record the time-of-flight spectra a multi channel analyser (manu: Fast-Comtec, TOF 7885) in connection with a memory module (MCD/PC) was used. The signal from the rotatable detector was preamplified with two fast preamplifiers (manu: Ortec, type:VT 120 , amplification $\times 20$ ), and then given through a discriminator (manu: Ortec, type: 436) to obtain a defined pulse shape and suppress noise. The output of the discriminator was fed to the TOF module which can handle a maximum of 65,536 channels with $5 \mathrm{~ns}$ channel width. The signal was then transferred by the MCD/PC module to a PC, where the actual TOF spectrum was displayed and updated every few seconds.

\subsubsection{Laser adjustment}

The laser beams are positioned in the reaction chamber with a vertically movable mask with pinholes for the laser position. It is connected to a mechanical counter outside the chamber over a rotary feedthrough and could thus be easily inserted into the laser beams. Here, one unit on the counter corresponds to $2.8 \cdot 10^{-3} \mathrm{~mm}$ movement. Once one absolute value of the counter was known for e.g. the pinholes in height of the skimmers and the lens, every position could be reproduced.

The diameter of the laser beams were also determined with the mask. The pinholes of the mask had a diameter of $0.7 \mathrm{~mm}$. A Gaussian beam profile was assumed for the laser beams and the laser beam diameter was defined as the width where the intensity has fallen to $1 / \mathrm{e}^{2}$. The energies of the laser pulses were measured with and without the mask inserted in the beam path. The fraction of the energy transmitted through the pinhole gave the diameter. For a Gaussian beam profile, the relation is given by eq. 5.3 [1].

Similarly to the detector surface, the beam diameter was enlarged compared to the hydrogen exchange reaction $(1.5 \mathrm{~mm}$ instead of $1.0 \mathrm{~mm})$. This enlarges the detection volume and hence the absolute number of particles which can be detected. This is of course only effective if the probe laser has a high enough photon density that with a larger diameter and thus a smaller density, the detection probability is still unity, which is the case here.

$$
\frac{P_{t}}{P_{i}}=1-e^{\left(-2 a^{2} / w^{2}\right)}
$$




$$
\text { with } \begin{aligned}
\mathrm{P}_{t} & : \text { transmitted power } \\
\mathrm{P}_{i} & : \text { initial power } \\
2 \mathrm{a} & : \text { pinhole diameter } \\
2 \mathrm{w} & : \text { beam diameter } 1 / \mathrm{e}^{2}
\end{aligned}
$$

\section{Bibliography}

[1] Demtroeder. Laserphysik. Springer-Verlag, 1977. 


\section{Chapter 6}

\section{Estimate of the total Signal}

To have an idea of the feasibility of realizing the reaction $\mathrm{OH}+\mathrm{D}_{2} \longrightarrow \mathrm{HOD}+\mathrm{D}$ first an estimate of the expected signal was made. To do so, the results from the hydrogen exchange reaction were taken as a basis. Since it was expected that the reactive D atoms would be less, already some measure were taken to improve the number of D atoms by simply enlarging the sensitive area of the detector and the probe volume as described in the chapter 5 .

The estimate of the signal starts with the density of particles $n$ in a pulsed valve expansion [1] (table 6.1):

$$
n=n_{o} \cdot 0.157 \cdot\left(\frac{d}{x}\right)^{2}
$$

with $x$ the distance from the nozzle opening and $d$ the effective nozzle opening, which is 6 times smaller than the real physical orifice of the valve. This factor was determined by a comparison of calculated and real number of reactive D atoms in the hydrogen exchange reaction. Using this factor, congruence of the calculated with the experimental results was achieved.

In case of an effusive beam which was also used within this work, the density $n$ is $n=n_{o} \cdot 10^{-3}$ at a distance of $5-10 \mathrm{~mm}$ from the surface of the source.

The density $n_{\text {sec }}$ of the secondary beam from the dissociation process in the reaction volume is given by

$$
\begin{array}{r}
n_{\text {sec }}=n_{\text {prec }} \cdot V_{D} \cdot P_{D} \cdot 1 / V_{K} \cdot q \cdot f_{v} \\
\text { with } P_{D}=\frac{n_{\text {phot }}}{A_{\text {Diss }}} \cdot \sigma_{a b s}
\end{array}
$$




\begin{tabular}{|l||c|c|c|}
\hline molecule / reaction & $\begin{array}{c}p_{o} \\
{[\mathrm{mbar}]}\end{array}$ & $\begin{array}{c}n_{o} \\
{\left[\mathrm{~cm}^{-3}\right]}\end{array}$ & $\begin{array}{c}n_{\text {prec }} \\
{\left[\mathrm{cm}^{-3}\right]}\end{array}$ \\
\hline $\mathrm{HNO}_{3}$ & 1000 & $2 \cdot 10^{19}$ & $5 \cdot 10^{13}$ \\
$\mathrm{H}_{2} \mathrm{O}_{2}$ pulsed, assumed & 100 & $2 \cdot 10^{18}$ & $2 \cdot 10^{12}$ \\
$\mathrm{H}_{2} \mathrm{O}_{2}$ pulsed, real & 20 & $4 \cdot 10^{17}$ & $4 \cdot 10^{11}$ \\
$\mathrm{H}_{2} \mathrm{O}_{2}$ effusive & 3 & $6 \cdot 10^{16}$ & $6 \cdot 10^{13}$ \\
$\mathrm{HI}$ for $\mathrm{H}+\mathrm{D}_{2}$ & 1000 & $2 \cdot 10^{19}$ & $5 \cdot 10^{13}$ \\
\hline
\end{tabular}

Table 6.1: Density $n_{\text {prec }}$ of $\mathrm{OH}$ precursor molecules in the nozzle beam. $p_{o}$ is the partial pressure of the molecule in the stagnation pressure, $n_{o}$ the number of precursor molecules in the stagnation pressure.

with $n_{\text {prec }}$ the density of the precursor molecules in the dissociation volume, $V_{D}$ the dissociation volume, $P_{D}$ the dissociation probability, $1 / V_{K}$ is a factor which describes the expansion of the molecules in a spherical shell, which is the same for all processes and is solely given by the distance of $30 \mathrm{~mm}$ between dissociation and reaction volume. $f_{v}$ is taking into account the velocity distribution of the fragments. If the distribution is rather broad, as it is the case for e.g. $\mathrm{HNO}_{3}$, the spherical shell is not given by the initial diameter of the dissociation volume, but by the real velocity distribution from the dissociation process. $f_{v}$ results from the velocity group used in the experiment. It can be chosen by the timing between dissociation laser and $\mathrm{D}_{2}$ beam and $\mathrm{D}$ detection. $\mathrm{q}$ is a measure for the quantum yield of the different dissociation channels.

For $\mathrm{H}_{2} \mathrm{O}_{2}$, initially expected values are included and also the real ones, which are adapted to the experimentally achieved vapour pressure of only 20 mbar in the gas phase above the solution instead of 100 mbar. Note that the amount of $\mathrm{H}_{2} \mathrm{O}_{2}$ in the resulting beam is even less, here only the lower vapour pressure with regard to the impossibility of heating a highly concentrated $\mathrm{H}_{2} \mathrm{O}_{2}$ solution as described in sec. 7.2.1 is considered.

The resulting density $n_{\text {sec }}$ of $\mathrm{OH}$ in the reaction volume is given in the table 6.2, the comparison is made with $\mathrm{H}$ from $\mathrm{HI}$, which is the precursor molecule in the hydrogen exchange reaction experiment.

The density $n_{D}$ of the reactively produced $\mathrm{D}$ atoms are given by the equation:

$$
n_{D}=n_{s e c} \cdot n_{D 2} \cdot v_{r e l} \cdot \sigma_{\text {reac }} \cdot \Delta t
$$

with $n_{D 2}$ the density of the $\mathrm{D}_{2}$ beam, $v_{\text {rel }}$ the relative velocity of the reaction partners, and $\sigma_{\text {reac }}$ the reaction cross section. The interaction time $\Delta t$ 


\begin{tabular}{|l||c|c|c|c|c|c|c|c|}
\hline $\begin{array}{l}\text { molecule } \\
/ \text { reaction }\end{array}$ & $\begin{array}{c}\mathrm{n}_{\text {prec }} \\
{\left[\mathrm{cm}^{-3}\right]}\end{array}$ & $\begin{array}{c}V_{D} \\
{\left[\mathrm{~cm}^{-3}\right]}\end{array}$ & $\begin{array}{c}\sigma_{a b s} \\
{\left[\mathrm{~cm}^{2}\right]}\end{array}$ & $P_{D}$ & $\begin{array}{c}1 / V_{K} \\
{\left[\mathrm{~cm}^{-3}\right]}\end{array}$ & $f_{v}$ & $\mathrm{q}$ & $\begin{array}{c}n_{\text {sec }} \\
{\left[\mathrm{cm}^{-3}\right]}\end{array}$ \\
\hline $\mathrm{HNO}_{3}$ & $5 \cdot 10^{13}$ & 0.01 & $1 \cdot 10^{-17}$ & 1 & $1 / 11.3$ & $1 / 40$ & 0.3 & $3.3 \cdot 10^{8}$ \\
$\mathrm{H}_{2} \mathrm{O}_{2}$ pulsed, assumed & $2 \cdot 10^{12}$ & 0.01 & $6 \cdot 10^{-19}$ & 0.37 & $1 / 11.3$ & $1 / 5$ & 1.5 & $2.3 \cdot 10^{8}$ \\
$\mathrm{H}_{2} \mathrm{O}_{2}$ pulsed, real & $4 \cdot 10^{11}$ & 0.01 & $6 \cdot 10^{-19}$ & 0.37 & $1 / 11.3$ & $1 / 5$ & 1.5 & $4.6 \cdot 10^{7}$ \\
$\mathrm{H}_{2} \mathrm{O}_{2}$ effusive & $6 \cdot 10^{13}$ & 0.01 & $6 \cdot 10^{-19}$ & 0.37 & $1 / 11.3$ & $1 / 5$ & 1.5 & $5.6 \cdot 10^{9}$ \\
$\mathrm{HI}$ for $\mathrm{H}+\mathrm{D}_{2}$ & $5 \cdot 10^{13}$ & 0.005 & $2 \cdot 10^{-19}$ & 0.8 & $1 / 11.3$ & 1 & 0.66 & $1.2 \cdot 10^{10}$ \\
\hline
\end{tabular}

Table 6.2: The density $n_{\text {sec }}$ of $\mathrm{OH}$ radicals in the secondary beam. With $n_{\text {prec }}$ the density of the precursor molecules in the dissociation volume, $V_{D}$ the dissociation volume, $P_{D}$ the dissociation probability, $1 / V_{K}$ a factor which describes the expansion of the molecules in a spherical shell and $f_{v}$ a factor with regard to the velocity distribution of the fragments.

of $75 \mathrm{~ns}$ below is an experimental value. The included data labelled ' $\mathrm{HNO}_{3}$, throat-of-nozzle' refer to an approach by Davis et al. [2], which will be explained in the chapter 8 , but is included here for later reference.

\begin{tabular}{|l||c|c|c|c|c|c|}
\hline $\begin{array}{l}\text { molecule } \\
/ \text { reaction }\end{array}$ & $\begin{array}{c}\mathrm{n}_{\text {sec }} \\
{\left[\mathrm{cm}^{-3}\right]}\end{array}$ & $\begin{array}{c}n_{D 2} \\
{\left[\mathrm{~cm}^{-3}\right]}\end{array}$ & $\begin{array}{c}v_{\text {rel }} \\
{[\mathrm{m} / \mathrm{s}]}\end{array}$ & $\begin{array}{c}\sigma_{\text {reac }} \\
{\left[\mathrm{cm}^{2}\right]}\end{array}$ & $\begin{array}{c}\Delta t \\
{[\mathrm{~ns}]}\end{array}$ & $\begin{array}{c}n_{D} \\
{\left[\mathrm{~cm}^{-3}\right]}\end{array}$ \\
\hline $\mathrm{HNO}_{3}$ & $3.3 \cdot 10^{8}$ & $4 \cdot 10^{14}$ & 4600 & $0.43 \cdot 10^{-16}$ & 75 & $1.9 \cdot 10^{5}$ \\
$\mathrm{H}_{2} \mathrm{O}_{2}$ pulsed, assumed & $2.3 \cdot 10^{8}$ & $4 \cdot 10^{14}$ & 4600 & $0.43 \cdot 10^{-16}$ & 75 & $1.3 \cdot 10^{5}$ \\
$\mathrm{H}_{2} \mathrm{O}_{2}$ pulsed, real & $4.6 \cdot 10^{7}$ & $4 \cdot 10^{14}$ & 4600 & $0.43 \cdot 10^{-16}$ & 75 & $2.8 \cdot 10^{4}$ \\
$\mathrm{H}_{2} \mathrm{O}_{2}$ effusive & $5.6 \cdot 10^{9}$ & $4 \cdot 10^{14}$ & 4600 & $0.43 \cdot 10^{-16}$ & 75 & $3.1 \cdot 10^{6}$ \\
$\mathrm{HI}$ for H+D $_{2}$ & $1.2 \cdot 10^{10}$ & $4 \cdot 10^{14}$ & 11280 & $0.25 \cdot 10^{-16}$ & 75 & $8.4 \cdot 10^{6}$ \\
\hline $\mathrm{HNO}_{3}$, throat of nozzle & $7.5 \cdot 10^{11}$ & $1 \cdot 10^{13}$ & 4120 & $0.22 \cdot 10^{-16}$ & 75 & $6.4 \cdot 10^{6}$ \\
\hline
\end{tabular}

Table 6.3: The density $n_{D}$ of the reactively produced $D$ atoms with $n_{D 2}$ the density of the $D_{2}$ beam, $v_{\text {rel }}$ the relative velocity of the reaction partners, and $\sigma_{\text {reac }}$ the reaction cross section, taken from ref. [3].

The number $N_{D}$ of detected $\mathrm{D}$ atoms is then given as follows:

$$
N_{D}=n_{D} \cdot V_{N} \cdot P_{N} \cdot I(\Omega) \cdot \Delta \Omega_{d e t}
$$

$V_{N}$ is the detection volume and $P_{N}$ the detection probability. $I(\Omega)$ denotes the expansion of the $\mathrm{D}$ atoms in spherical shell to the detector, and $\Delta \Omega_{\text {det }}$ the detector extension. $P_{N}$ is approximately 1 and $I(\Omega)=\frac{1}{4 \pi \cdot 30.5^{2} \mathrm{~cm}^{2}}$ in all cases.

Now the resulting number $N_{D}$ of detected $\mathrm{D}$ atoms per laser pulse can be taken from the table 6.4 . 


\begin{tabular}{|l||c|c|c|c|}
\hline $\begin{array}{l}\text { molecule } \\
\text { / reaction }\end{array}$ & $\begin{array}{c}\mathrm{n}_{D} \\
{\left[\mathrm{~cm}^{-3}\right]}\end{array}$ & $\begin{array}{c}V_{N} \\
{\left[\mathrm{~cm}^{-3}\right]}\end{array}$ & $\begin{array}{c}\Delta \Omega_{\text {det }} \\
{\left[\mathrm{cm}^{2}\right]}\end{array}$ & $N_{D}$ \\
\hline $\mathrm{HNO}_{3}$ & $1.9 \cdot 10^{5}$ & $5.7 \cdot 10^{-3}$ & 5 & 0.5 \\
$\mathrm{H}_{2} \mathrm{O}_{2}$ pulsed, assumed & $1.3 \cdot 10^{5}$ & $5.7 \cdot 10^{-3}$ & 5 & 0.3 \\
$\mathrm{H}_{2} \mathrm{O}_{2}$ pulsed, real & $2.8 \cdot 10^{4}$ & $5.7 \cdot 10^{-3}$ & 5 & 0.07 \\
$\mathrm{H}_{2} \mathrm{O}_{2}$ effusive & $3.1 \cdot 10^{6}$ & $5.7 \cdot 10^{-3}$ & 5 & 7.6 \\
${\mathrm{HI} \text { for } \mathrm{H}+\mathrm{D}_{2}}^{\mathrm{HNO}_{3}, \text { throat of nozzle }}$ & $8.4 \cdot 10^{6}$ & $3.9 \cdot 10^{-3}$ & 1.25 & 3.5 \\
\hline
\end{tabular}

Table 6.4: Absolute number of detected $D$ atoms with $V_{N}$ the detection volume and $\Delta \Omega_{\text {det }}$ the active surface of the detector.

When using the effusive beam as mentioned, it may be possible to enlarge the amount of $\mathrm{OH}$ in the reaction volume by a factor of 9 by reducing the distance between nozzle and reaction volume, also a factor of 2 more $\mathrm{OH}$ radicals may be achieved by gently heating the set-up.

\section{Bibliography}

[1] J.B. Anderson and J.B. Fenn. The Physics of Fluids, 8:780, 1964.

[2] B.R. Strazivar, C. Lin, and H.F. Davis. Science, 290:389, 2000.

[3] S. Koppe, T. Laurent, P.D. Naik, H.-R. Volpp, and J. Wolfrum. Can. J. Chem., 72:615, 1994. 


\section{Chapter 7}

\section{OH Precursor}

In principle, quite a number of molecules exist which give $\mathrm{OH}$ radicals in a photodissociation process, the simplest of it is water. But only very few molecules give $\mathrm{OH}$ radicals with the desired qualities, starting with the most severe requirement, which is that the velocity must be high enough to achieve a collision energy above the barrier. Some of the molecules giving off $\mathrm{OH}$ are dangerous, difficult to handle, unstable or must be chemically prepared and purified prior to use. Mostly these substances are liquid at room temperature and do not have a very high vapour pressure. This means that only few molecules are left which can be considered at all.

Most of the mentioned problems apply also to the chosen and tested precursors, here a compromise was made between the disadvantages and the desired properties of the fragment $\mathrm{OH}$ radical.

In this work, two different molecules and their different preparations were examined as a precursor, and two different kinds of beams were studied.

\subsection{Precursor $\mathrm{H}_{2} \mathrm{O}_{2}$}

$\mathrm{H}_{2} \mathrm{O}_{2}$ is the precursor molecule of choice, because of its rather narrow state and thus velocity distribution and of its high percentage of energy $(>90 \%)$ going into translational energy $[1,2]$. The maximal achievable velocities obtained from the dissociation of $\mathrm{H}_{2} \mathrm{O}_{2}$ from various wavelengths is shown in the table 7.1. Also the absorption coefficients of $\mathrm{H}_{2} \mathrm{O}_{2}$ and the maximally achievable collision energies in the crossed beam set-up are given.

For the first experiments, $193 \mathrm{~nm}$ was chosen as the dissociation wavelength. The rotational distribution of the $\mathrm{OH}$ radicals is well known at this wavelength. The most probable velocity is the $4550 \mathrm{~m} / \mathrm{s}$, with $\mathrm{j}=11$ the high- 
est populated rotational quantum of the $\mathrm{OH}$ radical with a spread of $\Delta j=4$ and a $\beta$-parameter of $0.35[3,1,4,2,5,6,7,8]$. The resulting collision energy is then $0.3685 \mathrm{eV}$ which is substantially higher than the barrier to the reaction of $0.230 \mathrm{eV}$

\begin{tabular}{|c|c|c|c|c|}
\hline $\begin{array}{c}\lambda_{\text {diss }} \\
{[\mathrm{nm}]}\end{array}$ & $\begin{array}{c}\sigma_{\text {abs }}[9] \\
{\left[10^{-19} \mathrm{~cm}^{2}\right]}\end{array}$ & $\begin{array}{c}\mathrm{E}_{\text {kin }}(\mathrm{OH}) \\
{[\mathrm{eV}]}\end{array}$ & $\begin{array}{c}\mathrm{v}_{\max }(\mathrm{OH}) \\
{[\mathrm{m} / \mathrm{s}]}\end{array}$ & $\begin{array}{c}\mathrm{E}_{\text {col }} \\
{[\mathrm{kcal} / \mathrm{mol}]}\end{array}$ \\
\hline 266 & $\approx 1$ & 1.255 & 3760 & 7.0 \\
248 & 1.5 & 1.425 & 4000 & 7.7 \\
212 & 4 & 1.85 & 4500 & 9.4 \\
193 & 6 & 2.137 & 4900 & 10.8 \\
\hline
\end{tabular}

Table 7.1: $\mathrm{H}_{2} \mathrm{O}_{2}$ photodissociation as an $\mathrm{OH}$ source. Different dissociation wavelengths are shown together with their absorption cross section, the maximal velocity gained by the $\mathrm{OH}$ fragments and the resulting maximum collision energy $\mathrm{E}_{\text {col }}$. For comparison, the $\mathrm{OH}+\mathrm{H}_{2}$ reaction has an activation energy of $4.0 \mathrm{kcal} / \mathrm{mol}$, whereas the $\mathrm{OH}+\mathrm{D}_{2}$ has an activation energy of $5.3 \mathrm{kcal} / \mathrm{mol}$ [10].

\section{$7.2 \quad \mathrm{H}_{2} \mathrm{O}_{2}$ from a pulsed valve}

The molecular beam of $\mathrm{H}_{2} \mathrm{O}_{2}$ seeded in $\approx 1$ bar of He are produced by expanding the mixture with a pulsed valve into vacuum and skimming the resulting beam.

Because $\mathrm{H}_{2} \mathrm{O}_{2}$ decays on surface contact, a special valve was constructed which allowed permanent flow, meaning that it consists of the valve itself, an external supply line and the normal internal line. The valve body was a standard part (manu: General Valves, series no. 9-500-900), the lower part with the orifice (orifice diameter: $0.5 \mathrm{~mm}$ ) was built locally by the mechanical workshop. On the supply line, He was bubbled through either liquid $\mathrm{H}_{2} \mathrm{O}_{2}$ in water or was conducted over an urea/hydrogen peroxide compound. The internal line was used to pump slightly on the valve, which allowed exhausted gas to be replaced by a fresh mixture. Exhausted gas means here that $\mathrm{H}_{2} \mathrm{O}_{2}$ undergoes catalytic decay to water on surface contact, and therefore the $\mathrm{H}_{2} \mathrm{O}_{2}$ content of the gas volume in the valve decreases continuously. The supply line and the inside of the nozzle as well as the pumping line were all teflon covered to prevent the catalytic decay of the hydrogen peroxide, but it cannot be entirely suppressed by the teflon coating and the gas has to be replaced after some time. A steady flow at a constant stagnation pressure of 1 bar could be thus sustained. The valve is shown in fig. 7.1. 
The valve was centred onto the skimmer (manu.: Beam Dynamics, model 2, skimmer orifice $2.1 \mathrm{~mm}$, height: $26.65 \mathrm{~mm}$ ) at a variable distance by guiding rods. Usually, the distance between the $\mathrm{H}_{2} \mathrm{O}_{2}$ valve and skimmer was $23 \mathrm{~mm}$. The distance from the $\mathrm{H}_{2} \mathrm{O}_{2}$ skimmer orifice to the point where the dissociation takes place is $32.6 \mathrm{~mm}$.

The temporal and spatial profile of the molecular beam pulses were measured by a fast ionization gauge (manu: Beam Dynamics, type: FIG-1, FH-1 gauge head) which is attached to the detector mount and can be therefore moved into the beams. Distance between gauge head and probe laser axis was then $217 \mathrm{~mm}$.

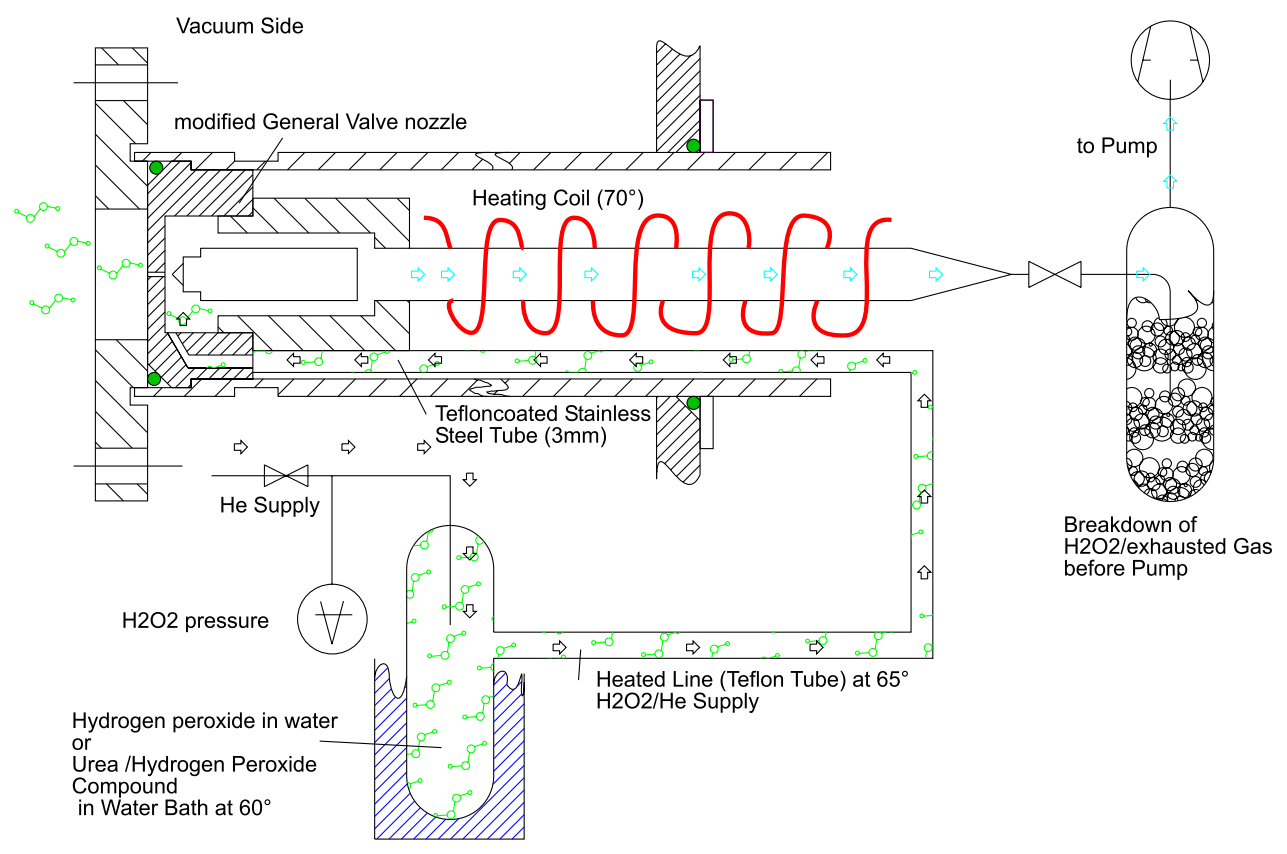

Figure 7.1: The specially designed permanent flow valve. The valve body is a standard part from General Valve, the lower part with the supply line and the nozzle is made by the mechanical work shop in Bielefeld.

\subsubsection{Preparation of $\mathrm{H}_{2} \mathrm{O}_{2}$ from an aqueous solution}

$\mathrm{H}_{2} \mathrm{O}_{2}$ from a aqueous solution is usually concentrated at $30 \%$. Up to this percentage, it is stable, but it is possible to obtain a higher concentration by slightly pumping on it. This can enhance the concentration up to $95 \%$ $[8,11]$. This concentration was actually necessary because $\mathrm{H}_{2} \mathrm{O}$ has a vapour pressure 10 times higher than $\mathrm{H}_{2} \mathrm{O}_{2}$, and the maximum ratio of $\mathrm{H}_{2} \mathrm{O}_{2}: \mathrm{H}_{2} \mathrm{O}$ is 1.3:0.9 in the vapour. 
The gas mixture of $\mathrm{H}_{2} \mathrm{O}_{2}$ and $\mathrm{He}$ was then produced by bubbling $\mathrm{He}$ through the solution.

The partial pressure of $\mathrm{H}_{2} \mathrm{O}_{2}$ at $30^{\circ} \mathrm{C}$ is given as 3 mbar [12] above the surface of the solution, thus it was also necessary to heat the solution up to $70-100{ }^{\circ} \mathrm{C}$, to produce a acceptable partial pressure of 50-100 mbar. During the experiments, when trying to heat up to this temperature, the decay within the solution appeared much more rapidly than anticipated. Also, it was not possible to heat the solution over $\approx 80^{\circ} \mathrm{C}$ because then it started to boil which reduced it to mere water.

In total, one can say that it was very difficult to balance the increased temperature to obtain higher vapour pressure against the increasing decay.

\subsubsection{Preparation of $\mathrm{H}_{2} \mathrm{O}_{2}$ from urea/hydrogen perox- ide compound}

Because $\mathrm{H}_{2} \mathrm{O}_{2} / \mathrm{H}_{2} \mathrm{O}$ is explosive and corrosive and, as it showed, the solution is not stable at higher temperatures, it was additionally tried to prepare and use $\mathrm{H}_{2} \mathrm{O}_{2}$ from an an urea hydrogen peroxide compound [manu: SigmaAldrich). This kind of $\mathrm{H}_{2} \mathrm{O}_{2}$ supply was already successfully used in IR spectroscopy [13]. There, the authors state that up to $85^{\circ} \mathrm{C}$, the urea itself does not decay but only gives off the hydrogen peroxide. To be on the safe side, the temperature was kept at $70-75^{\circ} \mathrm{C}$. The preparation itself is rather easy, the $\mathrm{H}_{2} \mathrm{O}_{2} /$ urea compound is placed in heated glass vessel and $\mathrm{He}$ is passed over it.

\subsection{3 $\mathrm{H}_{2} \mathrm{O}_{2}$ to $\mathrm{HO}_{2}+\mathrm{H}$ channel}

To improve the nozzle performance, it was tried to verify the $\mathrm{H}_{2} \mathrm{O}_{2}+\mathrm{h} \nu \longrightarrow \mathrm{HO}_{2}+\mathrm{H}$ channel, which is reported to have a quantum yield of $\Phi=0.16$ at $193 \mathrm{~nm}$ [6]. Cell experiments up to that point showed that $\mathrm{H}$ atoms with a kinetic energy release of $1.5 \mathrm{eV}$ with a FMHW of $0.39 \mathrm{eV}$ was produced. If it showed in the beam experiment that sharp peaks in the velocity distributions prevailed, it would be possible to use the $\mathrm{H}$ atoms from the dissociation to monitor and improve the nozzle performance.

Firstly, during the reaction the ratio $\mathrm{H}_{2} \mathrm{O}_{2} / \mathrm{H}_{2} \mathrm{O}$ could be determined without the LIF set-up, since the hydrogen detection scheme would be available anyway, second the opening time of the nozzle could be monitored and optimised that way, analogous to the hydrogen exchange reaction. There, hydrogen atoms from the precursor dissociation were detected in the reaction volume by tuning the Rydberg tagging from $\mathrm{D}$ atoms to $\mathrm{H}$ atoms. 
But the availability of sharp peaks is a condition which is necessary, since during the reaction the detection will take place $30 \mathrm{~mm}$ away from the nascency volume and thus only certain velocity groups can be detected. If the distribution is a structureless blob, the $\mathrm{H}$ atoms are equally distributed over a long time spread $(\approx 20 \mu \mathrm{s})$ with a low overall intensity, and it is impossible to discriminate against background hydrogen atoms. This dissociation process is not only important as an experimental tool, but also of interest in atmospheric chemistry, since the $\mathrm{HO}_{2}$ is considered important in atmospheric reaction chains.

When using urea/hydrogen peroxide compound as a precursor supply, the distribution of $\mathrm{H}$ atoms showed two superimposed features, shown in fig. 7.2. The peak at $1.27 \mathrm{eV}$ can be assigned to the dissociation of $\mathrm{H}_{2} \mathrm{O}$.

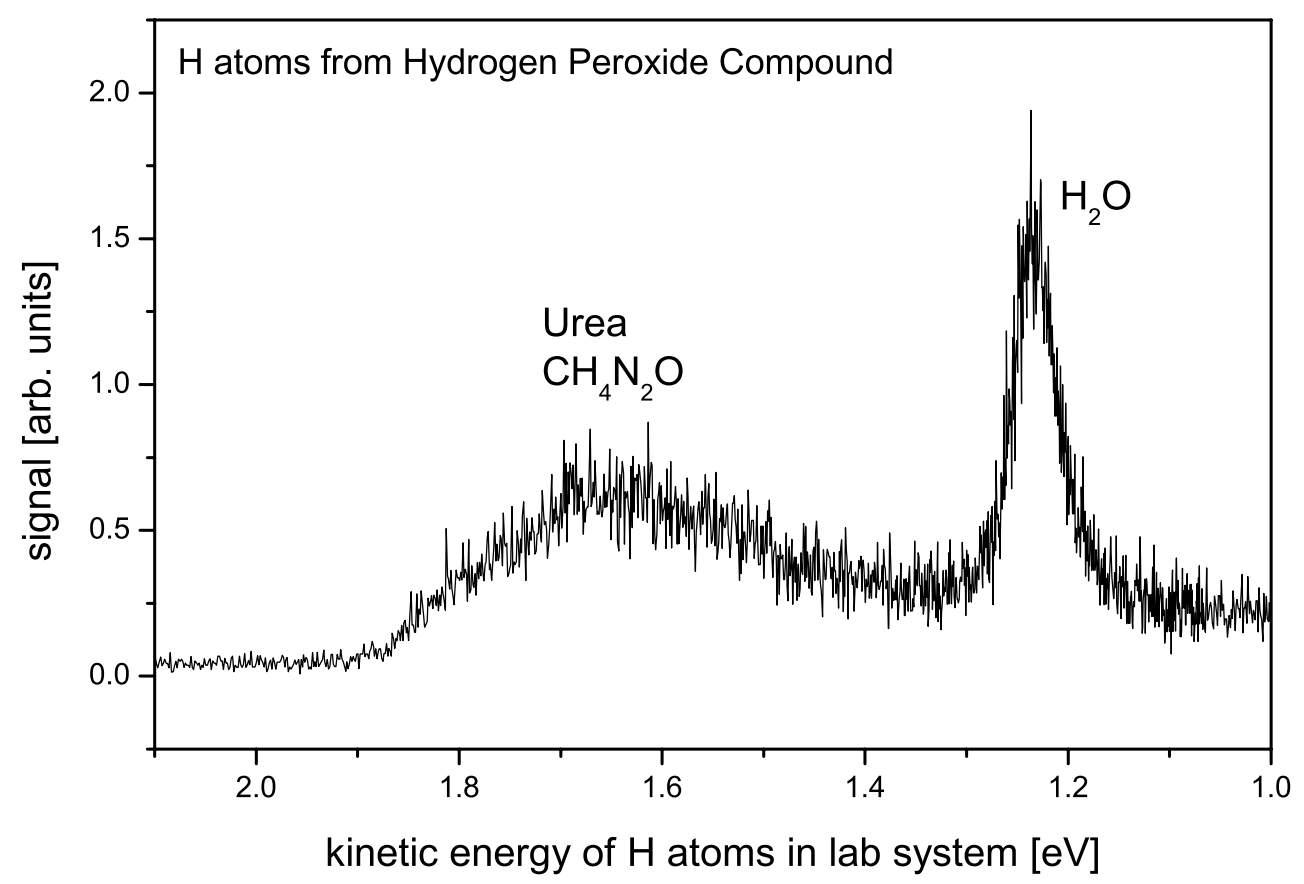

Figure 7.2: Hydrogen atoms from the urea/hydrogen peroxide compound at $193 \mathrm{~nm}$ dissociation wavelength. Initially, the $\mathrm{H}_{2} \mathrm{O}_{2} \longrightarrow \mathrm{HO}_{2}+\mathrm{H}$ channel was supposed to be measured. Later it showed that the two features of the distribution could be reproduced with the dissociation of the indicated molecules, water and urea itself.

The broader distribution with a maximum at $1.69 \mathrm{eV}$ indicates with its high energy onset a dissociation energy of $4.5 \mathrm{eV}$ which is not in agreement with to the dissociation energy of $\mathrm{H}_{2} \mathrm{O}_{2}$ at $3.8 \mathrm{eV}$. It showed that the broader distribution could be reproduced with pure urea. 
It was shown in other experiments that urea does not decay below $85^{\circ} \mathrm{C}$. The typical thermal decay product of urea which can give off $\mathrm{H}$ atoms is $\mathrm{NH}_{3}$. A comparison with dissociation experiments on $\mathrm{NH}_{3}$ at $193 \mathrm{~nm}$ showed no agreement, thus it can be confirmed that urea does not decay at that temperature and it must be concluded that the hydrogen atoms were dissociation products of the urea itself. That the urea itself is very efficiently suffused in the seed gas and can contribute significantly to the species in the beam and thus to experimental results was not reported in the literature about the IR spectroscopy experiments [13]. Quite likely this is because they were only probing the $\mathrm{OH}$ fragment, where any other fragments will not show, but in future studies the possible interaction with the urea in the beam has to considered.

The results when trying to measure the channel with $\mathrm{H}_{2} \mathrm{O}_{2}$ from an aqueous solution also mainly showed $\mathrm{H}$ atoms from the well known water dissociation.

\subsubsection{LIF from $\mathrm{H}_{2} \mathrm{O}_{2}$}

In the LIF spectrum of the $\mathrm{OH}$ fragments from hydrogen peroxide in aqueous solution (fig. 7.3), the typical rotational quanta were found at the dissociation at $193 \mathrm{~nm}(\mathrm{j}=9-11$, q1-branch), but also an high amount of population of small rotational states, $(j=3-5$, p1-branch), which are typical for the dissociation of water and thus an indication that the hydrogen peroxide decays more then expected. Since the absorption cross section of water is smaller by one to two orders of magnitude, depending of the internal excitation, this means that the amount of $\mathrm{H}_{2} \mathrm{O}$ in the beam is probably by the same order higher than the amount of $\mathrm{H}_{2} \mathrm{O}_{2}$. The resulting percentage of $\mathrm{H}_{2} \mathrm{O}_{2}$ molecules in the beam compared to $\mathrm{H}_{2} \mathrm{O}$ is then between 1 and $10 \%$. The water in the beam itself is not a problem, but the high amount of it shows that the beam contains less $\mathrm{H}_{2} \mathrm{O}_{2}$ than anticipated. Initially it was expected that at least $50 \%$ of the $\mathrm{H}_{2} \mathrm{O}_{2}$ in the stagnation gas volume reaches the resulting beam. Since in the concentrated solution $(\approx 95 \%)$ of $\mathrm{H}_{2} \mathrm{O}_{2}$ in $\mathrm{H}_{2} \mathrm{O}$ the resulting vapour pressures of $\mathrm{H}_{2} \mathrm{O}_{2}$ and $\mathrm{H}_{2} \mathrm{O}$ are roughly the same, the amount of $\mathrm{H}_{2} \mathrm{O}_{2}$ should be $25 \%$ of the amount of $\mathrm{H}_{2} \mathrm{O}$.

When performing the LIF experiments with $\mathrm{H}_{2} \mathrm{O}_{2}$ from the urea compound, one problem became apparent, which seriously impaired the usability

of the urea compound as a precursor supply. It showed that the $\mathrm{OH}$ signal dropped rather quickly during a measurement. When starting the experiments, we assumed that the $\mathrm{H}_{2} \mathrm{O}_{2}$ was gradually detached from the urea, but experiments showed a high response at the beginning (order of few min- 
utes), and then the signal declined. This means that either the $\mathrm{H}_{2} \mathrm{O}_{2}$ decayed quickly, or, if continuous pumping was maintained, that the compound was already depleted and the remaining release too small to maintain a continuous supply from the urea. Since the time needed to record a TOF spectrum in reaction experiments like they were performed in Bielefeld is of the order of an hour, the use of the urea compound does not seem feasible here.

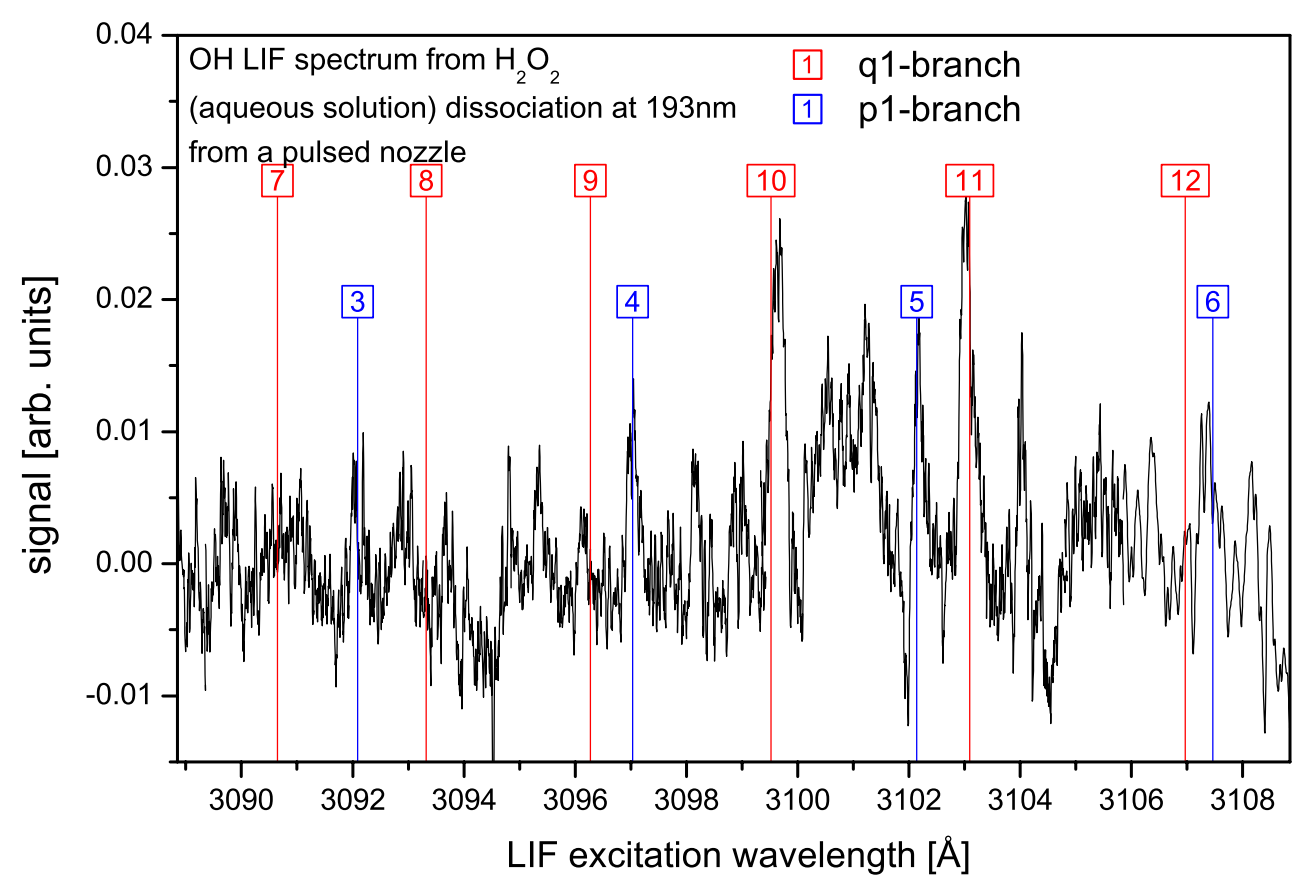

Figure 7.3: LIF spectrum of the $\mathrm{OH}$ from $\mathrm{H}_{2} \mathrm{O}_{2}$ dissociation at $193 \mathrm{~nm}$ wavelength. The $\mathrm{H}_{2} \mathrm{O}_{2}$ was obtained from an aqueous solution. The typical rotational quantum numbers around $j=11$ are populated (q1-branch), which indicates dissociation of $\mathrm{H}_{2} \mathrm{O}_{2}$, but also $\mathrm{OH}$ with small rotational quantum numbers $j=3-5$ (p1-branch) is present in the same order of magnitude, which shows that also $\mathrm{H}_{2} \mathrm{O}$ dissociation occurs. The two different branches were used because when combined they covered the low and middle $j$ values in the same wavelength range.

\subsection{Results from $\mathrm{H}_{2} \mathrm{O}_{2}$ with an effusive beam}

A different possibility which was investigated is the use of a effusive beam, which can be realized with a multi channel source. Effusive beams were quite common as molecular beam sources. They were replaced by continuous 
or pulsed valves, because these can produce in principal higher densities, narrower angular and velocity distributions and a efficient cooling of internal degrees of freedom (expansion), giving a higher transitional energy.

This is all true, but mainly applies to gases with high vapour pressure. For gases with small vapour pressure, the effusive beam has certain advantages. In this group, the photodissociation of tert-butyl-nitrite was experimentally investigated with the use of an effusive source. The fragment NO could be detected collisionfree a couple of $\mathrm{cm}$ away from the dissociation place by LIF.

The effusive source consists of a slitsource $(0.5 \times 22 \mathrm{~mm})$ which is covered with a piece of a disused multi channel plate ( $\mathrm{L}=500 \mu \mathrm{m}$ thickness $)$, which was cut to fit the opening. The MCP has hexagonally arranged cylindrical channels with a diameter of $2 \mathrm{R}=10 \mu \mathrm{m}$ and a distance between the centres of the channel of $11 \mu \mathrm{m}$. The slit source was slightly tilted by 7 degree to make up for the fact that the channels in a microchannel plate are tilted by that amount to avoid ion feedback and achieve good gain.

The detailed theory of the effusive beam is given in the references [14, 15]. Mainly, the mean free path plays an important role. If the stagnation pressure is low enough that the main free path $\Lambda$ is longer than the thickness of the plate which is here the length of the channels $\mathrm{L}$, the interaction between the molecules can be neglected. Thus, only a certain set of molecules pass through the channels, these are the molecules which have a velocity with a high component in the direction parallel to the channels. The resulting effusive beam mirrors this peaked velocity distribution. Thus the source itself does not direct the molecules, but cuts out a sample of molecules which have this direction anyway. The transmisson probability is given by

$$
W=\frac{8 R}{3 L}=0.027
$$

The peaking factor $W^{-1}$ is a measure of the ratio of the molecule intensity on the centerline in comparison to all effusive particles. The longer the channel $\mathrm{L}$, the smaller is $\mathrm{W}$ and thus the less the background pressure, which lessens the pumping requirements.

If an effusive beam is operated in opaque mode, which means that $\mathrm{R}<$ $\Lambda<\mathrm{L}, \mathrm{W}$ becomes a more complicated function and the directional peaking is reduced with increasing stagnation pressure.

The mean beam velocity is given by [14]

$$
\bar{v}_{\text {beam }}=\sqrt{8 k T / M \pi} .
$$


When using 1 mbar tert-butyl nitrite, a density of $10^{-3}$ mbar $\left(10^{14}\right.$ particles per $\mathrm{cm}^{3}$ ) was measured at a distance of $5-10 \mathrm{~mm}$ away from the multi channel plate surface. To achieve this kind of density with a pulsed $\mathrm{H}_{2} \mathrm{O}_{2}$ source, the backing pressure of $\mathrm{H}_{2} \mathrm{O}_{2}$ must be 1 bar, which simply cannot be achieved. Now the $\mathrm{H}_{2} \mathrm{O}_{2}$ vapour of 3 mbar at room temperature could be directly used without seeding in He and additional heating.

In the experiments with tert-butyl nitrite, a background pressure of $10^{-5}$ mbar resulted in a chamber which was pumped on with $60001 / \mathrm{sec}$. So we could assume that the background pressure would be uncritical in this experiment too. Even light heating of the valve can be considered. With warming the solution up to $50 \mathrm{deg}, 12 \mathrm{mbar}$ are achieved, 4 times more than the $3 \mathrm{mbar}$ at room temperature. Because in the opaque mode of the effusive beam, the density grows proportionally to the square root of the backing pressure, the amount of $\mathrm{OH}$ can be improved by a factor of 2 .

Thus the density of $\mathrm{H}_{2} \mathrm{O}_{2}$ in the effusive beam is higher then the expected density of $\mathrm{H}_{2} \mathrm{O}_{2}$ in the pulsed valve. Because of the low temperature, also the problem of the unstable solution at high temperatures is solved.

Because the dissociation takes now place at room temperature, the rotational distribution of the $\mathrm{OH}$ radicals is slightly broader, $\Delta j=6$ instead of $\Delta j=4$. Therefore the collision energy is slightly less defined $\left(\mathrm{E}_{c o l}=0.3686 \pm\right.$ $0.033 \mathrm{eV}$ instead of $\mathrm{E}_{c o l}=0.3686 \pm 0.02 \mathrm{eV}$ ).

Because the overall density is still small (no seed gas required), secondary collisions are rare and the effusive beam may be moved closer to the reactive volume.

Important is that the $\mathrm{H}_{2} \mathrm{O}_{2}$ density is not too high to make secondary collisions important. At a distance of $30 \mathrm{~mm}$ from the $\mathrm{D}_{2}$ beam, the density is below $10^{13}$ particles $/ \mathrm{cm}^{3}$. At a cross section of $10^{-15} \mathrm{~cm}^{2}$ the mean free path is still around $1 \mathrm{~m}$. This is sufficient compared to the pathway of $\mathrm{D}$ atoms to the detector of $30 \mathrm{~cm}$.

The actual LIF spectrum (fig. 7.4) shows that really the peaks are more pronounced than in the LIF spectrum from the pulsed nozzle. Rotational quantum numbers $\mathrm{j}=6-13$ can be made out. On the whole it is an improvement compared to the results from the pulsed nozzle, but still more $\mathrm{OH}$ was expected. In comparison to the results achieved with $\mathrm{HNO}_{3}$ (see the following section), the numbers are too low by a factor of approximately 20 , since the amount of $\mathrm{OH}$ was calculated to be higher by a factor of 10 , but is approximately only half of what is achieved in $\mathrm{HNO}_{3}$ dissociation.

Here, because it is a continuous source, the gas in the source is always replaced by fresh one, thus decay within the nozzle and the lines is probably not the biggest problem. But although it was attempted to passivate the channels within the microchannel plate of the source with teflon spray, most 


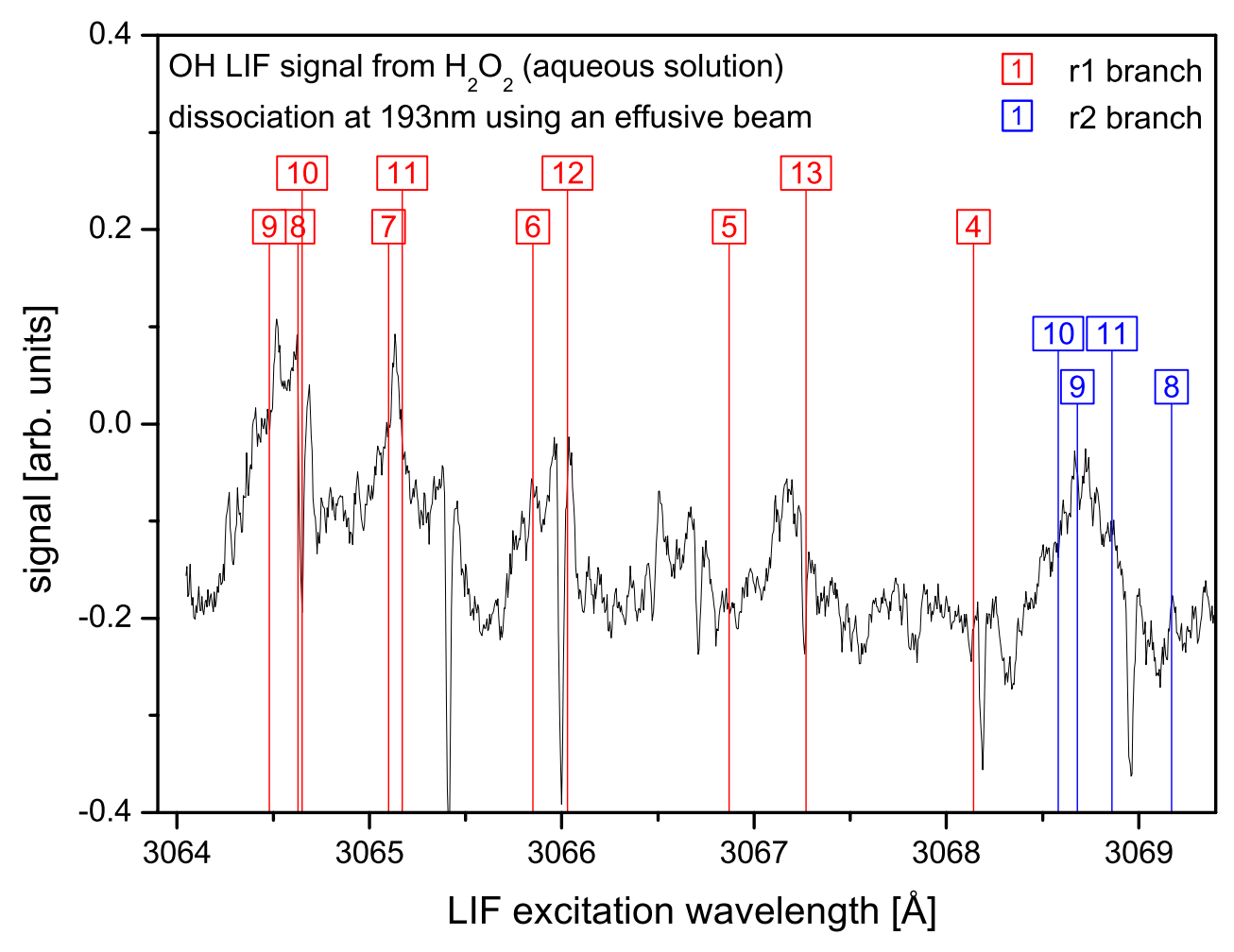

Figure 7.4: $\mathrm{OH}$ LIF spectrum from $\mathrm{H}_{2} \mathrm{O}_{2}$ in aqueous solution in an effusive beam at $193 \mathrm{~nm}$ dissociation wavelength. The $\mathrm{OH}$ peaks at around $j=10$ are a little more pronounced than in the pulsed beam case, but still the amount of $\mathrm{OH}$ is not sufficiently high.

decay probably happens in the channels.

\subsection{Precursor $\mathrm{HNO}_{3}$}

$\mathrm{HNO}_{3}$ is a precursor which has the advantage of giving a high amount of $\mathrm{OH}$ radicals in the fragmentation process, but only a small percentage of these radicals possess a high kinetic energy to overcome the barrier to the reaction.

At a temperature of $70^{\circ} \mathrm{C}, \mathrm{HNO}_{3}$ already has a vapour pressure of 1 bar. With an absorption coefficient of $\sigma=2 \cdot 10^{-17} \mathrm{~cm}^{2}$ this results in 10-20 times more $\mathrm{OH}$ if an decay rate of maximal $50 \%$ is assumed for $\mathrm{H}_{2} \mathrm{O}_{2}$. But because the velocity distribution ranges between 0 and $5000 \mathrm{~m} / \mathrm{s}$ with a maximum intensity at $2000 \mathrm{~m} / \mathrm{s}(3500 \mathrm{~m} / \mathrm{s}$ is minimum velocity to effect the reaction), thus the effectively usable amount of $\mathrm{OH}$ radicals is much smaller.

Felder et al. [16] measured the velocity distribution of $\mathrm{OH}$ radicals from 


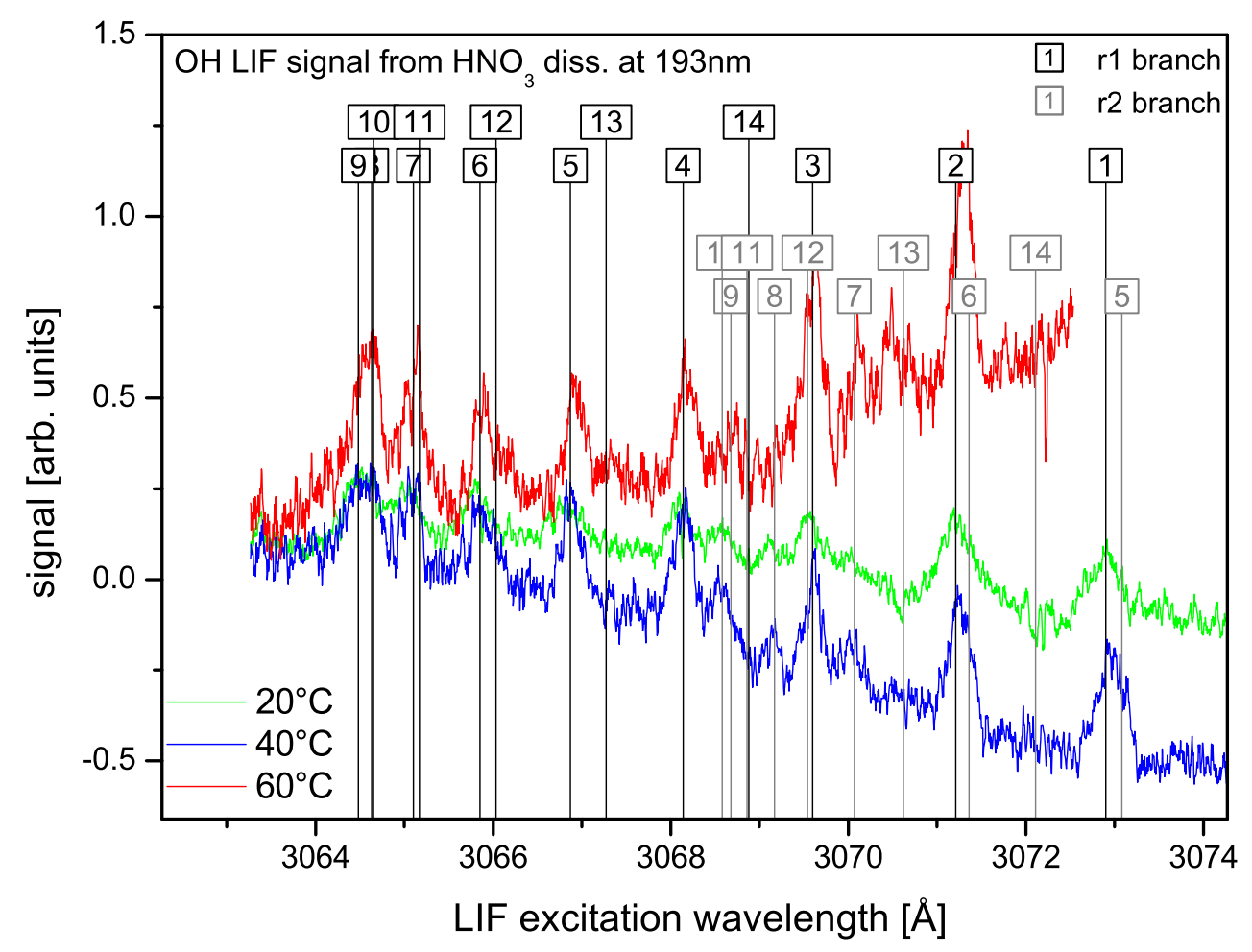

Figure 7.5: $\mathrm{HNO}_{3}$ dissociation at $193 \mathrm{~nm}$ at different temperatures. Quite clearly the LIF signal rises with increased temperature and thus pressure, proving that the molecule is stable with increased temperature.

the $\mathrm{HNO}_{3}$ dissociation at $193 \mathrm{~nm}$. If we consider that only $\mathrm{OH}$ radicals which are in the volume made up by the crossing of the $\mathrm{OH}$ radical beam, the $\mathrm{D}_{2}$ beam and the probe laser can contribute to the reaction at all, we obtain a velocity window of $750 \mathrm{~m} / \mathrm{s}$ at $4500 \mathrm{~m} / \mathrm{s}$ mean velocity. This window exist because $\mathrm{OH}$ from the lowest part of the dissociation volume can collide with $\mathrm{D}_{2}$ in the upper part of the reaction volume, which means they have the longest path and thus must have a higher velocity than the average. If it is the other way round, lower velocities are needed. With a fit to the velocity distribution from Felder et al. [16], the amount of $\mathrm{OH}$ within the window was determined to be $1 / 40$ of the total amount of $\mathrm{OH}$ fragments. Thus the usable number of $\mathrm{OH}$ fragments compared with the $\mathrm{OH}$ fragments from the $\mathrm{H}_{2} \mathrm{O}_{2}$ should be lower by a factor of $0.25-0.5$. After the experiments with $\mathrm{H}_{2} \mathrm{O}_{2}$ showed that the decay rate is much higher and only $1-10 \%$ of the $\mathrm{H}_{2} \mathrm{O}_{2}$ survive in the beam, the $\mathrm{OH}$ available for the reaction from $\mathrm{HNO}_{3}$ can be up to 10 times more than from $\mathrm{H}_{2} \mathrm{O}_{2}$. A small advantage is also that the angular $\mathrm{OH}$ distribution is slightly more peaked $\left(\beta=0.6 \pm 0.1\left(\mathrm{HNO}_{3}\right)[16]\right.$ 
instead of $\left.0.35\left(\mathrm{H}_{2} \mathrm{O}_{2}\right)[8]\right)$, which allows to direct the peaked portion into the $\mathrm{D}_{2}$ beam by turning the polarization of the dissociation laser accordingly.

A disadvantage is that because of the broad energy distribution, a worse energy resolution of the reaction products is to be expected. Because of the geometry of the experiment, it is possible that $\mathrm{OH}$ radicals with a velocity spread within $4125-4875 \mathrm{~m} / \mathrm{s}$ would be involved in the reaction, which gives a collision energy as broad as $0.3061-0.4121 \mathrm{eV}$. This was the reason why much time was spent to make the $\mathrm{H}_{2} \mathrm{O}_{2}$ dissociation feasible. The great differences in the reacting $\mathrm{OH}$ come from the different nascency places and that because of the great spread the used velocities are not peaked at one value but are evenly distributed within the usable window, contrary to the $\mathrm{H}_{2} \mathrm{O}_{2}$ case where the velocity distribution is peaked.

Of course, from the LIF spectrum (fig. 7.5) the velocities cannot be taken, since the excitations of the partner fragment also determines the total kinetic energy release. But from the LIF spectra we learn that the rotational quantum numbers $\mathrm{j}=1-13$ are populated. In the figure $7.5, \mathrm{OH}$ LIF spectra for $\mathrm{HNO}_{3}$ are shown at three different temperatures. It shows an increase in OH LIF signal with increasing temperature, but no significant change in the population of the rotational states is observed.

\section{Bibliography}

[1] K.H. Gericke, S. Klee, F.J. Comes, and R.N. Dixon. Jour. Chem. Phys., 85:4463, 1986.

[2] G. Ondrey, N. van Veen, and R. Bersohn. Jour. Chem. Phys., 78:3732, 1983.

[3] M.P. Docker, A.Hodgson, and J.P. Simons. Farad. Discuss. Chem. Soc., $82: 25,1986$.

[4] K.H. Gericke. Phys. Rev. Lett., 60:561, 1988.

[5] A.Jacobs, M-Wahl, R.Weller, and J. Wolfrum. Appl. Phys. B, 42:173, 1987.

[6] G.L. Vaghjiani, A.A. Turnipseed, R.F. Warren, and A.R. Ravishankara. Jour. Chem. Phys., 96:5878, 1992.

[7] D. W. Schwenke. Jour. Chem. Phys., 89:2076, 1988.

[8] A.U. Grunewald. Dynamik der Photofrgamenitierung von $\mathrm{H}_{2} \mathrm{O}$ und $\mathrm{H}_{2} \mathrm{O}_{2}$. Dissertation, Johann Wolfgang Goethe Universität zu Frankfurt am Main, Fachbereich Chemie, 1989. 
[9] H. Okabe. Photochemistry of small molecules. John Wiley and Sons, New York, 1978.

[10] A.R. Ravishankara, J.N. Nicovitch, R.L. Thompson, and F.P. Tully. Jour. Phys. Chem., 85:2498, 1981.

[11] K.H. Gericke. Private communication.

[12] Ullmann's Encyclopedia of Industrial Chemistry, Electronic Release. Wiley-Vch., 1998.

[13] M. Petterson, S. Tuominen, and M. Räsänen. Jour. Phys. Chem., 101:1166, 1997.

[14] H. Pauly. Other low energy beams. In G. Scoles, editor, Atomic and Molecular Beam Methods, Vol. 1, chapter 4. Oxford University Press, Oxford New York Toronto, 1988.

[15] W. Steckelmacher, R. Strong, and M.W. Lucas. Jour. Phys. D - Appl. Phys., 11:1553, 1978.

[16] P. Felder, X. Yang, and J.R. Huber. Chem. Phys. Lett., 215:221, 1993. 


\section{Chapter 8}

\section{Summary and Outlook}

The results obtained in the LIF spectra are mostly of a relative nature. Their OH LIF signals can be approximately compared with each other since the same lens set-up was used and the LIF laser intensity was stable and quite well reproducible. Minor problems were caused by the doubling crystal, but these could be solved online, i.e. during the measurements without effecting them considerably. But in case of the OH LIF measurements from $\mathrm{H}_{2} \mathrm{O}_{2}$ the signal to noise ratio was so bad that it was very difficult to distinguish between real peaks and small variations in the laser intensities and thus a quantitative analysis not feasible. Also fluctuations in the nozzle performance prevented an exact quantitative comparison. Thus the comparison was kept to determine the order of magnitudes between the signals, for which the reproducibility of the conditions was more than sufficient.

But the relative nature refers more to the fact that it was not possible to gauge the amount of $\mathrm{OH}$ against an known signal in this lens set-up, because the density of particles in the beam can only be estimated but not absolutely determined.

The measurement with $\mathrm{HNO}_{3}$ can kind of serve as a calibration measurement in comparison to the other LIF measurements because it is stable and the pressure can be reproducibly controlled with the temperature. Thus in comparison to the density of $\mathrm{HI}$ in the precursor beam a quite accurate estimate can be made.

The density of HI can be determined as described in chapter 6 by comparison of the calculated total amount of $\mathrm{D}$ atoms in the hydrogen exchange reaction with the really detected ones. From this number, the effective nozzle opening can be taken. Thus the density $n_{\text {prec }}\left(\mathrm{HNO}_{3}\right)$ given in table 6.1 is probably quite accurate within the frame of this approach to estimate it. Since the amount of $\mathrm{OH}$ was supposed to be on threshold of realizing the $\mathrm{OH}+\mathrm{D}_{2}$ reaction, the approximate comparisons to it as done for the effusive 
beam in the chapter 7 also give an idea of the factor by which the amount of $\mathrm{OH}$ is too low to effect the reaction. It is about a factor 2 less than from $\mathrm{HNO}_{3}$, but should be about a factor of 10 higher.

But the estimate that the amount of $\mathrm{OH}$ from $\mathrm{HNO}_{3}$ was on the verge of effecting the reaction is still only an estimate, and thus the relative nature of the results from the LIF spectra remains and could only be resolved by simply trying to realize the reaction with the tested beams. To use $\mathrm{H}_{2} \mathrm{O}_{2}$ in He from a pulsed nozzle was excluded because clearly the yield of $\mathrm{OH}$ was too poor. But both test with either the $\mathrm{H}_{2} \mathrm{O}_{2}$ effusive beam and with using $\mathrm{HNO}_{3}$ as a precursor did not give any D atom spectra which could be clearly assigned to the reaction.

Hence the assumption that the $\mathrm{OH}$ density in the reaction volume would not be high enough could be solidified. But I believe due to the complexity of the experiment, other problems may have also influenced the outcome. Although all care was taken that all other components were in good working order, some of them could not be easily tested independently, as there are the $\mathrm{D}_{2}$ beam or the gain of the detector, and may have worsened after the initial check. Especially the detector which is an secondary electron multiplier could have deteriorate quickly under the influence of the now present water molecules. These problem may not have been decisive in themselves, but in combination with the low $\mathrm{OH}$ yield a poorer sensibility may have been fatal.

In the quest for state resolved differential cross section Floyd Davis et al. of Cornell University, Ithaka later used a different approach [1] which was more successful. He used $\mathrm{HNO}_{3}$ in a novel photodissociation outline to produce $\mathrm{OH}$ a) in a sufficiently high amount, b) with enough kinetic energy to overcome the barrier to the reaction efficiently and c) a much narrower velocity distribution than normal dissociation of the $\mathrm{HNO}_{3}$. The achieved densities are also included in the signal estimates in chapter 6 . The main point of the approach is to dissociate the precursor within the expansion region of the molecular beam (throat of nozzle - dissociation). Davis used 40 mbar of $\mathrm{HNO}_{3}$ seeded in 2 bar $\mathrm{H}_{2}$. Because of the dissociation within the expansion region, the $\mathrm{OH}$ is kept within the beam by collisions with the $\mathrm{H}_{2}$. Because now all fragments form part of the expanding beam, the kinetic energy obtained in the expansion by cooling the internal degrees of freedom and the energy generated in the dissociation process $\left(\mathrm{E}_{\text {excess }}=\right.$ $4.3 \mathrm{eV}$ ) are evenly distributed or levelled out by the collisions so that roughly all molecules in the beam have the same velocity. This is basically the same as a heavier molecule seeded in a lighter gas when the heavier molecule is already present in the gas stagnation volume. He gives the achieved density 
as $n_{O H}=7.5 \cdot 10^{11}$ at a distance of $7.6 \mathrm{~cm}$ from the nozzle, assuming all $\mathrm{OH}$ stays in the beam, and a mean velocity of $3340 \mathrm{~m} / \mathrm{s}$.

The main advantage is that a relatively high amount of $\mathrm{OH}$ is produced from a stable precursor and brought to a uniform and high enough velocity, so all $\mathrm{OH}$ can be used. But because this is still rather low to overcome the barrier, the $\mathrm{D}_{2}$ beam is not a pure $\mathrm{D}_{2}$ beam but also seeded in $\mathrm{H}_{2}$. The collision energy than reaches $0.286 \mathrm{eV}(6.6 \mathrm{kcal} / \mathrm{mol})$ which is just enough to overcome the barrier of $0.230 \mathrm{eV}(5.3 \mathrm{kcal} / \mathrm{mol})$. The seeding of the $\mathrm{D}_{2}$ in $\mathrm{H}_{2}$ is of course is a disadvantage compared to the experimental idea in Bielefeld. He uses 1 bar of $\mathrm{D}_{2}$ in 4 bar of $\mathrm{H}_{2}$. In the experiments here, 9 bar o- $\mathrm{D}_{2}$ at liquid nitrogen temperature was used. Firstly, this cools the rotational distribution so effectively that $97 \%$ of the $D_{2}$ is in $j=0$, which allows a high energy resolution and also a clear assignment of the obtained differential cross section. This is helpful when comparing with theoretical results, because they are normally calculated with a total angular momentum $\mathrm{J}=0$ of the reactants. Secondly, apart from these qualitative arguments, it simply gives a very high density of $\mathrm{D}_{2}$ molecules. A pressure of 9 bar at liquid nitrogen temperature is approximately equivalent to 30 bar $\mathrm{D}_{2}$ at room temperature, and hence a 30 times higher density of $\mathrm{D}_{2}$ molecules can be achieved.

Because of this difference in the $\mathrm{OH}$ beam production, the $\mathrm{OH}$ beam is not perpendicular to the precursor beam as it had been the case in Bielefeld, but coincides with it. Thus the the approach of Davis requires that the two initial beams are set at an angle to each other. A perpendicular geometry was chosen.

Their results showed vibrational resolution. The distribution found confirmed the findings by Casavecchia, which were not vibrationally resolved but nevertheless indicated that a large amount $(\approx 66 \%)$ of the available energy, which is composed of the initial collision energy $(0.274 \mathrm{eV})$ and of the high exoergicity $(0.656 \mathrm{eV})$ of the reaction is deposited into HOD internal excitation and that the HOD products are strongly backwards scattered.

The findings by Davis et al. showed that mainly one and two quanta of the OD stretching mode are excited $((0,1)$ and $(0,2))$. Contribution of the $(1,1)$ mode was also present, and a very small contribution with no excitation at all $(0,0)$. As the authors point out, the OD stretching mode $(0,1)$ is nearly the double bending frequency $(2,0)$, thus in an experiment, $(0,1)$ and $(2,0)$, $(1,1)$ and $(3,0)$, and $(2,1)$ and $(4,0)$ are energetically not distinguishable . Here only theoretical calculations can help to clarify. They predict that only the above mentioned four vibrational excitation combinations $((0,0)$, $(1,1),(0,1)$ and $(0,2))$ are significantly populated. Concerning the potential energy surface, they found that their result agreed extraordinarily well with calculation on the OC surface, in contrast to calculations on the Schatz- 
Elgersma (SE-PES) [2] and the Kliesch-Werner-Clary (KWC-PES) [3] surface. The KWC-PES at least gave rather highly excited vibrational states, even with too high a number of involved states, whereas the SE-PES tends more to populate low vibrational states.

At the moment, still theoretical calculations on $\mathrm{OH}+\mathrm{H}_{2} \rightleftharpoons \mathrm{H}_{2} \mathrm{O}+\mathrm{H}$ and its isotopic variants are under way (see references in chapter 4 ), but since Davis published his results no new experimental approaches on the reaction $\mathrm{OH}+\mathrm{H}_{2} \longrightarrow \mathrm{H}_{2} \mathrm{O}+\mathrm{H}$ or its isotopic variant have been performed.

\section{Bibliography}

[1] B.R. Strazivar, C. Lin, and H.F. Davis. Science, 290:389, 2000.

[2] G.C. Schatz and H. Elgersma. Chem. Phys. Lett., 73:21, 1980.

[3] A. Kliesch, H.-J. Werner, and D.C. Clary. unpublished. 


\section{Part II}

\section{$\mathbf{I}_{2}$ and $\mathbf{I}_{2}^{+}$Dissociation Dynamics}




\section{Chapter 9}

\section{Aims of this Work}

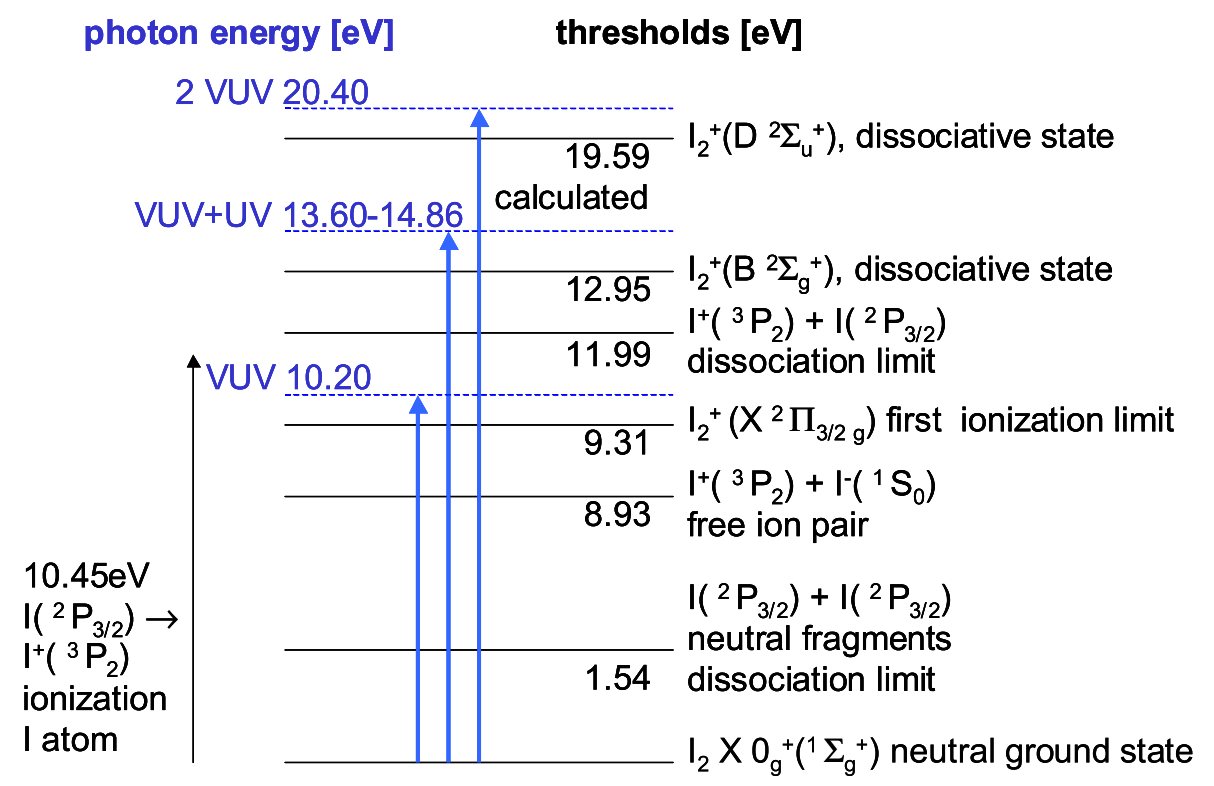

Figure 9.1: Some thresholds of the iodine molecule in comparison with the total photon energies employed in this work. The examples are chosen to illustrate the most important decay / dissociation processes addressed in this work. Energies are not to scale. Note that the minimum photon energy required within the Frank-Condon window are usually higher than the given dissociation limits.

This part of this work deals with dissociation, ionization and decay processes of the iodine molecule above the first ionization threshold, yielding I atoms, $\mathrm{I}_{2}^{+}, \mathrm{I}^{+}$and $\mathrm{I}^{-}$. Because of the range of UV lasers and the VUV beam which are available in this laboratory, excitation energies of several $\mathrm{eV}$ above 
the first ionization threshold of iodine can be realized. Within this range, only a very limited number of experiments have been performed. Especially the energy range between 13.60 and $14.86 \mathrm{eV}$ excitation energy has so far not been explored in detail.

To illustrate the energy range in which this work operates, some of the ionization and dissociation thresholds are listed in the figure 9.1 in comparison with the total photon energies employed, which are shown as blue arrows. The different transition energies are given by the horizontal lines. Included are the dissociation limits to neutral fragments, ion pair states and the first dissociation limit of the iodine ion $\mathrm{I}_{2}^{+}$. The first ionization limit as well as two transition energies to dissociative ionic states are given. The ionization energy of the iodine atom is also shown in comparison. The processes include one and two photon absorption, with the energy of one VUV photon lying above the threshold of ionization, neutral dissociation or dissociation to free ion pair states. With the absorption of two photons, either two VUV photons or a combination of VUV and UV photons, the energy is already higher than the transition energy to dissociative ionic states.

The molecular orbitals of iodine molecule are formed by the five $5 \mathrm{p}$ electrons of the atom, which give the $\sigma_{g}^{2} \pi_{u}^{4} \pi_{g}^{* 4} \sigma_{u}^{* 0}$ configuration of the molecular ground state. Although the $\mathrm{A}^{2} \Pi_{u}$ and $\mathrm{B}^{2} \Sigma_{g}^{+}$states of iodine cation have admixtures of configurations with one electron removed and one excited, the main configuration is one where one electron can be removed in a one photon transition.

The chapters of this work are organized as follows.

- Chapter 10 gives an overview of the literature so far concerning potentials of $\mathrm{I}_{2}$ and $\mathrm{I}_{2}^{+}$, both experimentally and theoretically.

- Chapter 11 introduces the experimental method, velocity map imaging. The following chapter 12 describes the alteration to the initial set-up necessary to implement this method.

- In chapter 13 , the photoelectron images are inspected in order to know which ionic (bound) states are present and may contribute to a subsequent dissociation.

- In chapter 14 the $\mathrm{I}^{+}$images at VUV wavelength were at first carefully analyzed in order to assign the processes to the various total excitation energies, which lie between $10.20 \mathrm{eV}$ (one VUV photon) and $20.40 \mathrm{eV}$ (two VUV photons). Then, in comparison with other experimental 
works and theoretical predictions, the analysis is carried over to discussion of the mechanism and involved potentials which may lead to these results.

- In chapter 15 , results obtained with a combination of one VUV and one UV photon are discussed. The total excitation energy achieved with this combination is between 13.60 and $14.86 \mathrm{eV}$. A unique process was found which so far has not been observed in the iodine cation at this wavelength. Here also the mechanisms are discussed and amendments to the existing potential curves are proposed.

- In chapter 16, the observed free ion pair channel is discussed.

- The last chapter 17 closes this work with a summary and an outlook. Additional experiments to further detail the iodine potentials are suggested. Recent advancements of the velocity map imaging techniques are described. 


\section{Chapter 10}

\section{Introduction to Iodine Potentials}

Mulliken published his review article 'Iodine revisited' in 1971 which summed up the research up to then and is one of the milestones in research on molecular iodine. His article includes detailed discussions of the results so far on the predissociating $\mathrm{B}^{3} \Pi\left(0_{u}^{+}\right)$state and other states deriving from the 2431 configuration, other valence states, emission spectra of the iodine vapour, the 1441 configuration which gives rise to absorption in the UV (Cordes band, $180-200 \mathrm{~nm}$ ) and the ion pair states. The configuration notations are given as $k l m n$, which indicates the populated molecular orbitals $\sigma_{g}^{k} \pi_{u}^{l} \pi_{g}^{* m} \sigma_{u}^{* n}$. He summerizes the transition energies, dissociation products and transition rules of the valence and ion pair states.

Venkateswarlu [1] published the first comprehensive study of the vacuum ultraviolet absorption spectrum, the assignments of which stood unchallenged for 30 years. Only in 2002 Ridley et al. published a reanalysis of the VUV absorption spectrum augmented by new measurements [2].

After Mulliken published his review, the Dalby [3, 4] and Goodman [5] systems were discovered which both belong to a system of Rydberg states. They were found at the multi photon ionization (MPI) wavelengths 363$378 \mathrm{~nm}$, band origin at $53562 \mathrm{~cm}^{-1}(6.642 \mathrm{eV})$ and $360-600 \mathrm{~nm}$, band origin at $48426 \mathrm{~cm}^{-1}(6.004 \mathrm{eV})$ respectively.

Because iodine has been the subject of these many spectroscopical studies throughout the last century, most of its basic characteristics are fairly well known. This makes it also a popular molecule to demonstrate the virtue of new techniques, either showing in general that the new technique works, or even leading to improved resolution and thus to more details of the spectroscopic quantities. A typical example for this are the extensive studies of 
Lawley, Donovan and Cockett on the iodine molecular Rydberg states and the spectroscopic data of the electronically and vibrationally excited states of $\mathrm{I}_{2}^{+}$with zero-kinetic energy pulsed field ionization $[6,7]$.

The Rydberg states have been a fertile ground of exploration anyway. The interaction of Rydberg states converging to the first ionization limit with ion pair states inspired a lot of research $[8,9,10,11]$, also the autoionizing Rydberg states based on excited ionic molecular states [12].

In recent years, the study of ion pair states of iodine [13] also drew more and more attention $[14,15,16,17,18,19,10,8,20,21,13,22,11,23,2]$. Although only one state, the $\mathrm{D}\left(0_{u}^{+}\right)$state, is directly accessible from the molecular ground state $\mathrm{X}^{1} \Sigma\left(0_{g}^{+}\right)$, most others can be reached with the $\mathrm{B}^{3} \Pi\left(0_{u}^{+}\right)$ state as an intermediate, so molecular constants are now known for most of them. Interesting enough, although the ion pair state channel is available to all molecules which are able to form a stable anion, only 50 species are known, where photodissociative free ion pair formation occurs [24].

But even with all this research undertaken, iodine still poses a lot of question, especially little is known of the ionized molecule. Photoelectron spectra to obtain ionization potentials were quite frequently examined, [25, $6,7,26,27,28,29,30,31,32]$, Mason et al. studied the $\mathrm{A}^{2} \Pi_{u}-\mathrm{X}^{2} \Pi_{g}$ emission spectrum [33], which was expected to be there since most $\mathrm{A}^{2} \Pi_{u}$ vibrational states are still below the $\mathrm{I}+\mathrm{I}^{+}$limit, but could not be observed until then. The $\mathrm{B}^{2} \Sigma_{g}^{+}$state also received much attention to determine its exact character as dissociating or predissociating state [34]. The bound states $\mathrm{X}^{2} \Pi_{g}$ and $\mathrm{A}^{2} \Pi_{u}$ are thus quite well characterized, but apart from the $\mathrm{B}^{2} \Sigma_{g}^{+}$state, little can be found about the higher dissociative states in literature, although many more are predicted.

Also few theoretical works exist which treat the potentials of $\mathrm{I}_{2}^{+}[35,36$, $37,38]$. Others species of the halogens had earned more interest, especially on the theoretical side, since iodine is with the highest mass the halogen which is most likely to experience extensive coupling of the higher states, which makes it the most difficult to calculate.

Except for predissociation of the $\mathrm{B}^{2} \Sigma_{g}^{+}$state which is quite well studied, only few experimental works exist which explore dissociation processes of the iodine cation, e.g. McLoughlin et al. studied the dissociation of the $\mathrm{A}^{2} \Pi_{u}$ states produced in electron bombardment [39], Tuckett et al. found experimentally that for the NeI $(16.85-16.67 \mathrm{eV})$ and $\mathrm{HeI}(21.22 \mathrm{eV})$ radiation, dissociative ionization occurs $[40,41]$. Similar results were found in a multiphoton absorption experiment [42]. 


\section{Bibliography}

[1] P. Venkateswarlu. Can. Jour. Phys., 48:1055, 1970.

[2] T. Ridley, D.A. Beattie, M.C.R. Cockett, K.P. Lawley, and R.J. Donovan. Phys. Cem. - Chem. Physics, 4:1398, 2002.

[3] G. Petty, C. Tai, and F.W. Dalby. Phys. Rev. Lett., 34:1207, 1975.

[4] F.W. Dalby, G. Petty-Sil, M.H.L. Pryce, and C. Tai. Can. Jour. Phys., 55:1033, 1977.

[5] K.K. Lehmann, J. Solarek, and L. Goodman. Jour. Chem. Phys., 69:1569, 1978.

[6] M.C.R Cockett, J.G. Goode, K.P. Lawley, and R.J. Donovan. Jour. Chem. Phys., 102:5226, 1995.

[7] M.C.R Cockett, R.J. Donovan, and K.P. Lawley. Jour. Chem. Phys., 105:3347, 1996.

[8] K.P. Lawley, T. Ridley, Z. Min, P.J. Wilson, M.S.N. Al-Kahali, and R.J. Donovan. Chem. Physics, 197:37, 1995.

[9] A. Kvaran, H. Wang, G.H. Johannesson, and A.J. Yencha. Chem. Phys. Lett., 222:436, 1994.

[10] A. Kvaran, A.J. Yencha, D.K. Kela, R.J. Donovan, and A. Hopkirk. Chem. Phys. Lett., 179:263, 1991.

[11] K.P. Lawley, A.C. Flexen, R.J. Maier, A. Manck, T. Ridley, and R.J. Donovan. Phys. Cem. - Chem. Physics, 4:1412, 2002.

[12] M.C.R Cockett, D.A. Beattie, N.A. Macleod, K.P. Lawley, T. Ridley, and R.J. Donovan. Phys. Cem. - Chem. Physics, 4:1419, 2002.

[13] S. Motohiro, A. Umakoshi, and T. Ishiwata. Jour. Molec. Spec., 208:213, 2001.

[14] H.P. Grieneisen and R.E. Francke. Chem. Phys. Lett., 88:585, 1982.

[15] C.J. Fecko, M.A. Freedman, and T.A. Stephenson. Jour. Chem. Phys., 116:1361, 2002.

[16] J.P. Perrot, M. Broyer, J. Chevaleyre, and B. Femelat. Jour. Molec. Spec., 98:161, 1983. 
[17] M.D. Wheeler, I.R. Lambert, and M.N.R. Ashfold. Chem. Phys. Lett., 229:285, 1994.

[18] U. Heeman, H. Knöckel, and E. Tiemann. Chem. Phys. Lett., 90:17, 1982 .

[19] J.C.D. Brand and A.R. Hoy. Can. J. Phys., 60:1209, 1982.

[20] P.J. Wilson, T. Ridley, K.P. Lawley, and R.J. Donovan. Chem. Physics, 182:325, 1994.

[21] P.J. Jewsbury, T. Ridley, K.P. Lawley, and R.J. Donovan. Jour. Molec. Spec., 157:33, 1993.

[22] D. Inard, D. Cerny, M. Nota, R. Bacis, S. Churassy, and V. Skorokhodov. Chem. Physics, 243:305, 1999.

[23] A. Hiraya, K. Shobataka, R.J. Donovan, and A. Hopkirk. Jour. Chem. Phys., 88:52, 1988.

[24] J. Berkowitz. In U. Becker and D.A. Shirley, editors, VUV and Soft X-ray Photoionisation. Plenum, New York, 1996.

[25] H. van Lonkhuyzen and C.A. Lange. Chem. Physics, 89:313, 1984.

[26] S. Evans and A.F. Orchard. Inorganica Chimica Acta, 5:81, 1971.

[27] A.J. Yencha, M.C.R Cockett, J.G. Goode, R.J. Donovan, A. Hopkirk, and K.P. Lawley. Chem. Phys. Lett., 229:347, 1994.

[28] B.R. Higginson, D.R. Lloyd, and P.J. Roberts. Chem. Phys. Lett., 19:480, 1973.

[29] D.C. Frost, C.A. Dowell, and D.A. Vroom. Jour. Chem. Phys., 46:4255, 1967.

[30] A.W. Potts and W.C. Price. Trans. Faraday Soc., 67:1242, 1971.

[31] A.B. Cornford, D.C. Frost, A. McDowell, J.L. Ragle, and I.A. Stenhouse. Jour. Chem. Phys., 54:2651, 1971.

[32] Y.-Y. Gu, A.M. Chojnacki, C.J. Zietkiewicz, A.A. Senin, and J.G. Eden. Jour. Chem. Phys., 119:12342, 2003.

[33] S.M. Mason and R.P. Tuckett. Chem. Phys. Lett., 160:575, 1989. 
[34] J.H.D. Eland. Jour. Chem. Phys., 70:2926, 1979.

[35] Junqing Li and K. Balasubramanian. Jour. Molec. Spec., 138:162, 1989.

[36] A.D. McLean, O. Gropen, and S. Huzinga. Jour. Chem. Phys., 73:396, 1980.

[37] W.A. de Jong, L. Visscher, and W.C. Nieuwpoort. Jour. Chem. Phys., 107:9046, 1997.

[38] J.M. Dyke, G.D. Josland, J.G. Snijders, and P.M. Boerrigter. Chem. Physics, 91:419, 1984.

[39] R.G. McLoughlin, J.D. Morrison, and D.L. Smith. Inter. Jour. Mass Spec. and Ion Proc., 58:201, 1984.

[40] R.P. Tuckett, E. Castellucci, M. Bonneau, G. Dujardin, and S. Leach. Chem. Physics, 92:43, 1985.

[41] S. Leach. Jour. Phys. Chem., 92:5373, 1988.

[42] S. Unny, Y. Du, L. Zhu, R.J. Gordon, A. Sugita, M. Kawasaki, Y. Matsumi, and Tamar Seidelman. Phys. Rev. Lett., 86:2245, 2001. 


\section{Chapter 11}

\section{Velocity Map Imaging}

In Bielefeld, we implemented Velocity Map Imaging (VMI) into the existing vacuum apparatus as a new technique because it allowed the simultaneous measurements of both speed and angle with a 2-dimensional detector, contrary to the Rydberg tagging TOF method or 1-dimensional REMPI measurements. The resulting advantages are higher signal and shorter measurement times, which not only increases the signal-to-noise ratio but also makes it easier to keep the laser and the molecular beam stable during one measurement. So far, only attempts on conventional ion imaging had been made in this group, which does not give the same detail and resolution as VMI.

\subsection{Principle of velocity map imaging}

Velocity map imaging has become during the last couple of years the stateof-the-art technique for the measurements in the field of photodissociation and is increasingly used in molecular reaction dynamics measurements.

At its basis is the general principle of ion imaging. In ion imaging measurements, contrary to one-dimensional methods as time-of-flight measurements and Doppler spectroscopy, the kinetic energy release information is not given in a temporal or wavelength profile, but as spatial information in a 2-dimensional image.

The aim of ion imaging is to produce an image or projection of the 3dimensional Newton sphere which allows its later reconstruction. Usually the source volume of the charged particles we wish to image lies within an electrostatic field which accelerates the cloud of particles in direction of the detector. After the electrostatic field is passed, a field free or low field drift path follows. On this drift path, the Newton sphere expands and then collapses onto the detector and appears as a $2 \mathrm{D}$ projection of the initial $3 \mathrm{D}$ 


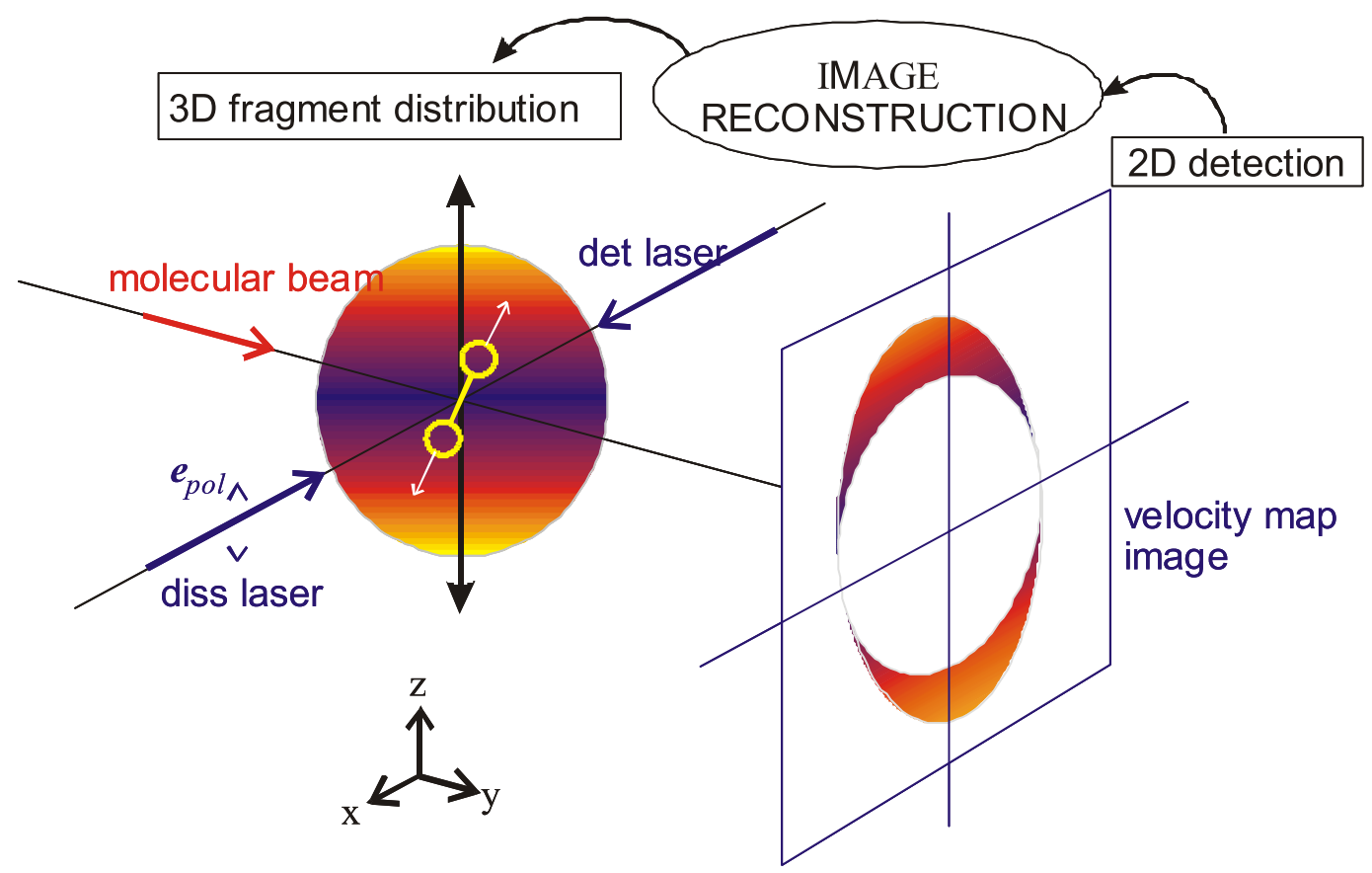

Figure 11.1: Principle of velocity map imaging. The molecular beam is crossed with a dissociation and if necessary, a probe laser. The polarization vector of the dissociation laser is in plane with the detector. The fragments are projected onto the 2-dimensional detector. The 3D-distribution can be reconstructed using the inverse Abel transform. Figure courtesy of A. Eppink.

distribution. The velocity of the fragments obtained from the dissociation process is mirrored in their distance from the centre of the image. Apart from the speed distribution (speed stands for the scalar, velocity for the vector quantity), which can be converted to kinetic energy release, also the angular distribution of the fragments is preserved in the image.

In conventional ion imaging experiments, as they were introduced by Chandler et al. $[1,2]$ grids were used to produce a homogeneous electrostatic field to preserve the initial 3-dimensional distribution of the photodissociation products without any disturbance and translate it onto the detector. But the grid electrodes always limited the quality of the images. They reduce the transmission, lead to trajectory deflections and cause blurring because of their non-point source geometry, since every grid-opening acts as a point source in itself.

The ion imaging method was improved by A. Eppink and D. Parker at the University of Nijmegen [3, 4]. Instead of using grids, they replaced the 
grids with an open electrode set-up which consists of three plates which form an electrostatic lens. The expression lens may be a bit misleading, since this 'lens' does not focus all the ions into one spot, which could be expected in analogy to an optical lens, but has ideally the following properties.

- The initial Newton sphere or nested spheres are compressed in the direction of the flight axis. This effect is called 'pancaking'. In this direction the velocity from the dissociation process is superimposed on the velocity in direction of the detector. This velocity is dominated by the acceleration by the electric field. Since this velocity is mass dependent, the compression of the 3D distribution in this direction allows a neat mass separation.

- The radial distance from the centre of the image is directly proportional to the speed obtained in the dissociation process by the fragments.

- All particles with the same velocity (speed and angle) are mapped on the same point independently of their origin within a certain source volume. This deblurring is actually a function of the voltage ratio applied to the electrostatic lens electrodes.

- Particles with a different mass, but the same kinetic energy release end up in the same radial distance from the centre. This was used to calibrate images with an unknown kinetic energy release.

The deblurring feature of the lens can very easily be influenced by the ratio of the voltage. Eppink et al. [4] found that the deblurring was best for a ratio of 0.71 of the extractor to repeller voltage. In this experiment, the ideal ratio was 0.72 . The principle of the deblurring is shown in figure 11.2, taken from reference [4].

Since all trajectories with the same velocity vector are mapped onto the same point, the expression velocity map imaging (VMI) was coined for this method.

The principle set up is shown in fig. 11.3 below. The figure shows the original setup as it is still in use in Prof. Parker's group in Nijmegen, figure courtesy of A. Eppink, but altered to include the appropriate wavelengths and molecular beams used in this experiment.

To the left the electrostatic lens made up of three electrodes is shown. The utmost left is called the repeller, the middle one the extractor plate. The last plate is grounded. The valve is here mounted on axis and the laser beams come in parallel to the electrodes in between the repeller and extractor plate. Eppink et al. mention that the resolution is best when the source volume is 


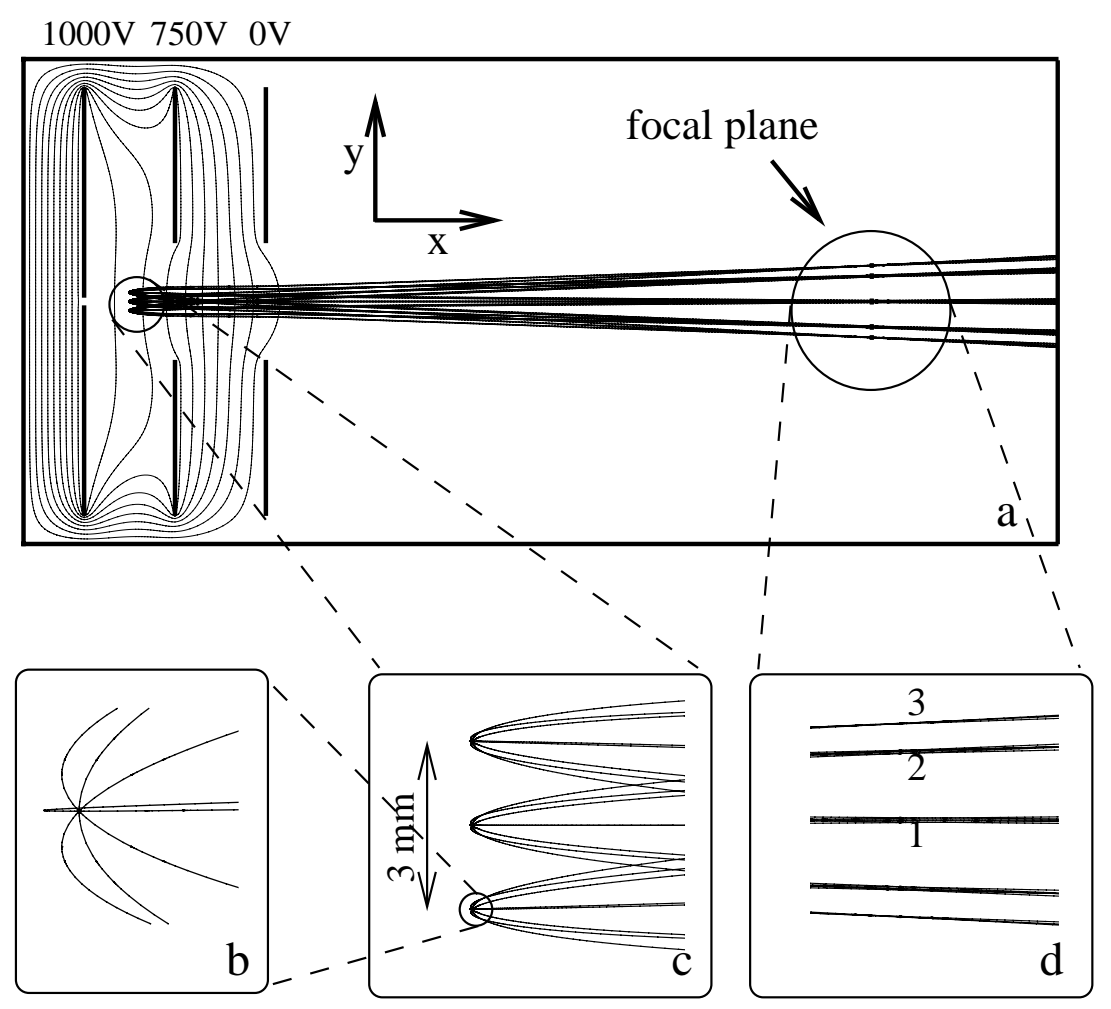

Figure 11.2: The deblurring property of the electrostatic lens. Simulated ion trajectories and equipotential lines are shown. Panel (a) gives an overview, whereas panel (b)-(d) illustrate the details. The line source of $3 \mathrm{~mm}$ in panel (c) is caused by a laser propagating along the $y$ axis. In each point along the line source, ions originate as shown in panel (b), with eight ion trajectories at angle spacing $45^{\circ}$ as examples. In panel (d) the focal plane is shown where trajectories with the same ejection angle but from different positions in the line source come together. Figure taken from reference [4].

equidistant from both plates. The ion cloud is then imaged onto the detector, which consists of a stack of micro channel plates and a phosphor screen, and subsequently, a CCD-camera.

\subsection{Event counting}

Event counting was also introduced by A. Eppink. It enhances the resolution tremendously and elegantly eliminates two experimental problems along the way. One problem is the noise of the camera, the other that most MCPs do not have a uniform amplification over the whole area. Of course this 


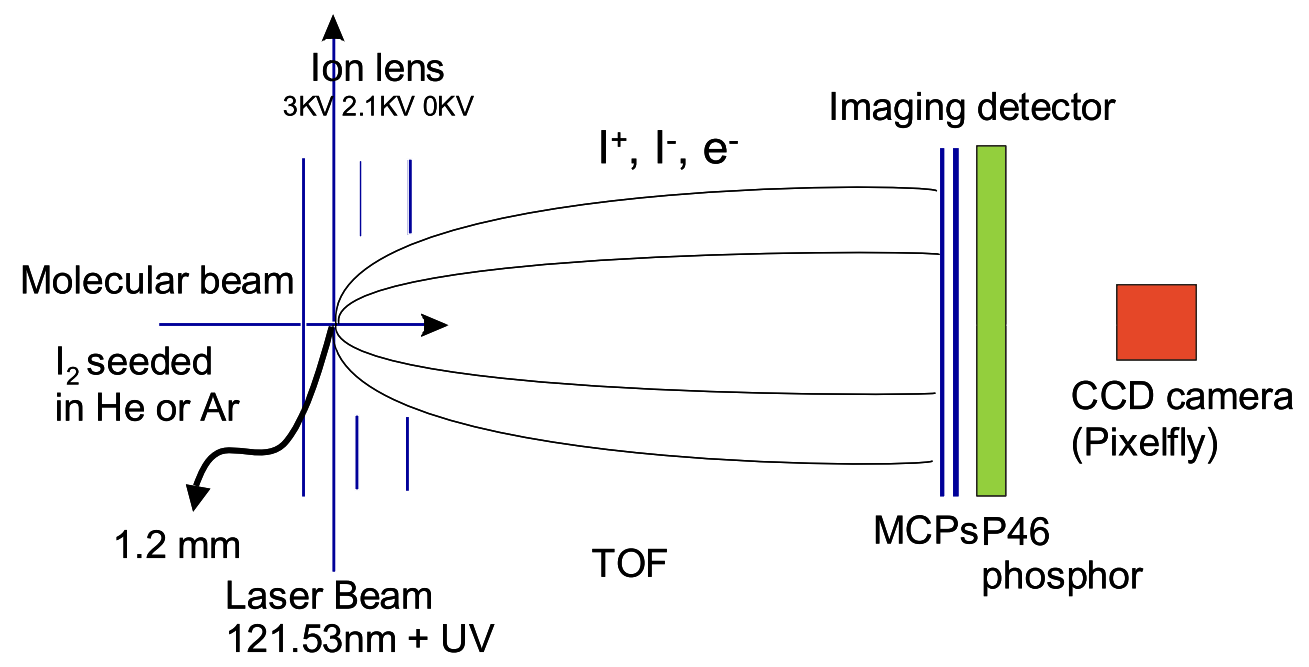

Figure 11.3: Velocity map imaging set-up.

problem can be partly solved by more sophisticated and in general, more expensive cameras, as well as more expensive MCPs which guarantee uniform amplification.

In the experiments the ion images of 8,000-20,000 laser shots were added up. Each shot gives 10-100 ions. Each ion produces a fluorescing spot on the phosphor screen which approximately comprised 4-9 pixels on the CCD chip and varied in intensity. If now these spots are added up, an image of the distribution is formed. But the resolution is than limited by the size of the dots, as well that in places where the gain of the MCP is bigger a higher intensity is created, which falsely makes the impression of a higher amount of ions in this region. As well the noise of the camera and of stray ions may add up to a significant amount.

The idea is that not the intensity of the light spots on the phosphor screen is added up by the counting routine, but that only light flashes above a certain threshold value are accepted as an event. From these flashes, which usually cover some pixels of the CCD camera, the centre is determined (centroiding) and stored as the location of one event caused by one ion. One event is characterized by a normalized intensity of one. If the threshold is carefully chosen, it eliminates not only the effects of any inequalities of the MCPs, which may have a better gain in places which would give much more weight to events there if the intensities were added up. As well it cancels out any background noise of the camera which is usually below the threshold. And most stray ions will not cause events above the threshold because of their lack of high kinetic energy which is needed to give rise to sufficiently intensive 
electron cloud in the MCPs. Still, some stray events outside the clear ring structure are observed. The eventcounting routine to be implemented into the DaVis software was kindly made available by A. Eppink.

The event counting routine is well suited for experiments with a low ion yield where every ion makes a singular spot clearly marked off from any other spot. If this is not given, the overall ion signal must be reduced which may influence the results. In this work, only occasionally the VUV power or the intensity of the molecular beam had to be adjusted to achieve this condition for the ion images. This means that the laser power was reduced by the order of $10-20 \%$. If the molecular beam was within the limit of the allowed gas load, the final adjustment could be of the order of $50 \%$. Only when the photoelectron images were taken the laser power had to be reduced greatly to obtain distinguishable spots on the phosphorscreen. The reduction was usually done with a stack of fused silica plates placed in the way of the UV laser, with a nominal transmission of $92 \%$ each. Thus a full stack of 10 gives an overall transmission of $44 \%$. Since some of the plates were old and absorbed more, the lowest transmission which could be achieved was probably much less than $44 \%$. The VUV light was then so low in intensity that it was not detectable by the NO-cell anymore (see section 3.2.1), thus only this estimate from the plates can be given.

\subsection{Abel inversion and BASEX expansion}

If a 3-dimensional distribution has a cylindrical symmetry, it is in principle possible to derive the 3D-distribution from its $2 \mathrm{D}$-projection. The $2 \mathrm{D}$ projection of a 3D-distribution parallel to its symmetry axis is called an Abel transformation, and the corresponding back-projection is than known as an inverse Abel transformation or Abel inversion.

To ensure the Abel inversion can be applied to the 2D projection of the initial 3D distribution, the axis of the symmetry must be in a plane parallel to the detector plane. Because the cylindrical symmetry of the angular distribution in photodissociation experiments is determined by the polarization axis of the incident laser beam, it follows that the polarization of the laser must lie in a plane parallel to the detector surface. Usually, the obtained 3D distribution is shown and represented by a vertical slice along the central axis through the 3D distribution, depicting the ion density in this plane. In general, this slice already contains all information. The final speed distribution can then be obtained by integrating over all angles within the 3D-distribution.

To understand how an Abel inversion is performed, we start with a 3D 
distribution $I(x, y, z)$ which has a cylindrical symmetry around the $z$ axis, which is identical with the polarization axis of the laser. The $2 \mathrm{D}$ projection is given by $\mathrm{I}(x, z)$. Now the problem can be resolved into group of a 1 dimensional (1D) intensity profile along one of the axes, i.e. the $x$ axis, where one 1D profile exists for each $z$ value, and each intensity profile can be handled separately. A line by line analysis of the image then gives $I_{z}(x, y)$ for each 1D profile $\mathrm{I}_{z}(x)$.

The inverse Abel transformation is now the reconstruction of the actually 2-dimensional, axially symmetric function $f(x, y)=f(r)$ from its projection $[5]$.

The projection onto the $\mathrm{x}$ axis can be described as follows:

$$
f(x)=\int_{-\infty}^{\infty} f(x, y) d y=2 \cdot \int_{0}^{\infty} f(r) d y
$$

with $r=\sqrt{x^{2}+y^{2}}$, it follows $r d r=x d x$ and the equation reads:

$$
f(x)=2 \cdot \int_{x}^{\infty} \frac{f(r) r d r}{\sqrt{r^{2}-x^{2}}}
$$

This equation is the Abel transform of $f(r)$. Since we are interested to obtain $f(r)$ from the projection, we have to use the inverse Abel transform $[2]$, which is:

$$
f(r)=-\frac{1}{\pi} \frac{\delta}{\delta r} \int_{r}^{\infty} \frac{r f(x) d x}{x \sqrt{x^{2}-r^{2}}}
$$

or $[6]$

$$
f(r)=-\frac{1}{\pi} \int_{r}^{\infty} \frac{(d f(x) / d x)}{\sqrt{x^{2}-r^{2}}} d x .
$$

But when using the Abel inversion, some problems arise. Firstly it is difficult to calculate due to its singularity at the lower limit. Secondly, because the derivative of the projection data is used, noise can cause an unproportional large error propagation. The first working idea was to reformulate the original Abel inversion as an inverse Hankel transform of the Fourier transform of the projected data, which has the advantage that it is easily implemented as well that filtering in the frequency domain can be applied after the Fourier transform. 
But the problem of retransforming the projected data to the original distribution has been addressed by numerous groups and scientists around the world since velocity map imaging started to evolve as the standard method. Matsumi et al. [7] introduced a back-projection method which included filtering in the frequency domain to reduce experimental noise, but at the cost of smoothing the data and thus losing information. Helm et al. also developed a back-projecting method [8, 9]. Loock and Manzhos [10] published an application based on the onion peeling algorithm. Vrakking presented an iterative approach to reconstruct the images [11]. A comparison of these different methods was made by A. Eppink et al.[12], which discusses the advantages and disadvantages of the various approaches in respect to different experimental methods and image qualities. Lately an attempt was made to directly measure the slice of the 3D distribution, which foregoes the laborious and error-prone transformation process. This will be discussed in chapter 17.3 .

Concerning inversion, the approach by Dribinski et al. [13], called Basis Function Expansion (BASEX), proved to be the most promising and was used in this work. The formulation is here as follows.

$z$ is again the axis of symmetry, $x$ is the axis perpendicular to $z$ and both axis form the detector plane. $r$ denotes the radial distance from the $z$-axis in 3-dimensional space. In the detector plane, $r$ and $x$ coincide. Because of the cylindrical symmetry, also the 3D image is actually a function of only two coordinates, $r$ and $z$, which gives $I(r, z)$. The projection onto the the detector plane formed by $z$ and $x$ is denoted $P(x, z)$.

Now it is assumed that a basis set of functions $\left\{f_{n}(r, z)\right\}$ exist which give

$$
I(r, z)=\sum_{n=0}^{N_{\max }} c_{n} f_{n}(r, z) .
$$

Because projecting is a linear operation, it can be concluded that

$$
P(x, z)=\sum_{n=0}^{N_{\max }} c_{n} g_{n}(x, z),
$$

with $g_{n}(x, z)$ the projection of $f_{n}(r, z)$.

Now it is possible to reconstruct the function $I(r, z)$ from the projection $P(x, z)$. 
1. The basis set $\left\{f_{n}(r, z)\right\}$ must be chosen so that expansion 11.5 exists. Therefore the set must be complete in the space of $I(r, z)$ functions.

2. The basis set of projections $\left\{g_{n}(r, z)\right\}$ is then generated. With cylindrical symmetry present, the projections are generated with the Abel transformation as above:

$$
g(x, z)=\int_{\|x\|}^{\infty} \frac{f_{n}(r, z) r d r}{\sqrt{r^{2}-x^{2}}} .
$$

This can be done for each $f_{n}(r, z)$ either numerically or preferably analytically.

3. To find the set of coefficients $\left\{c_{n}\right\}$, the experimentally measured projection $P(x, z)$ must be expanded in a series as equation 11.6.

4. With equation 11.5 and the set of coefficients $\left\{c_{n}\right\}$, the original 3Ddistribution $I(r, z)$ can be reconstructed.

In comparison with other methods, the BASEX method showed the very good results with noisy images and images with high dynamics. Also did it not produce any artefacts, which are common in the initial Hankel-Fourier transform. Another advantage is the computational requirements. The basis functions are Gaussians which do not need to be calculated especially for different images, thus they can be calculated once and for all and prior to the images. Then the inversion process actually only requires matrix operations to obtain the coefficients. The basis set and the matrix were combined by the authors in a small program and made available to other researchers. It was used throughout this work. The program makes available two sets of basis functions. Here the set with broader basis functions was used. The broader functions cover around 2 pixel instead of 1 . This is helpful especially in images with event counting, since because of the characteristics of the event counting routine, high pixel-to-pixel fluctuation can occur, because of the necessity to work at low signal and the correlation of one event to one normalized count in one pixel position, instead of an image, where large spots with smoothed out intensities are added up.

The only problem which could not be eliminated with the BASEX method is the centerline noise, although it is much less than in an Abel inverted image. To understand where the noise comes from, it is helpful to realize that each point of the original $3 \mathrm{D}$ distribution located at $r_{0}$ from the symmetry axis only contributes to points in the $2 \mathrm{D}$ projection which lie at $x \leq r_{0}$, which can be seen in equation 11.2. The result is that the image contains less information for regions nearer to the axis compared to regions farther 
out. Especially, only the points on the centre line of the projection contain information about the centre line of the original 3D-distribution, whereas information about the points with $r_{0}=r_{\max }$ are stored in every point of the 2D-distribution. Considering that the noise is in most cases evenly distributed over the images, it follows that the signal-to-noise ratio decreases when going towards the centre line and causes uncertainties along the central axis.

This problem was treated more successfully by an iterative method by Marc Vrakking [11], but this method has the disadvantage of being rather slow.

\subsection{Calibration of the images}

To obtain an energy / speed calibration of the images, the $\mathrm{I}^{+}$images were compared to an image of the dissociation of HI, where the dissociation channels are well known. The two images used for the initial calibration are shown in fig. 11.4 .

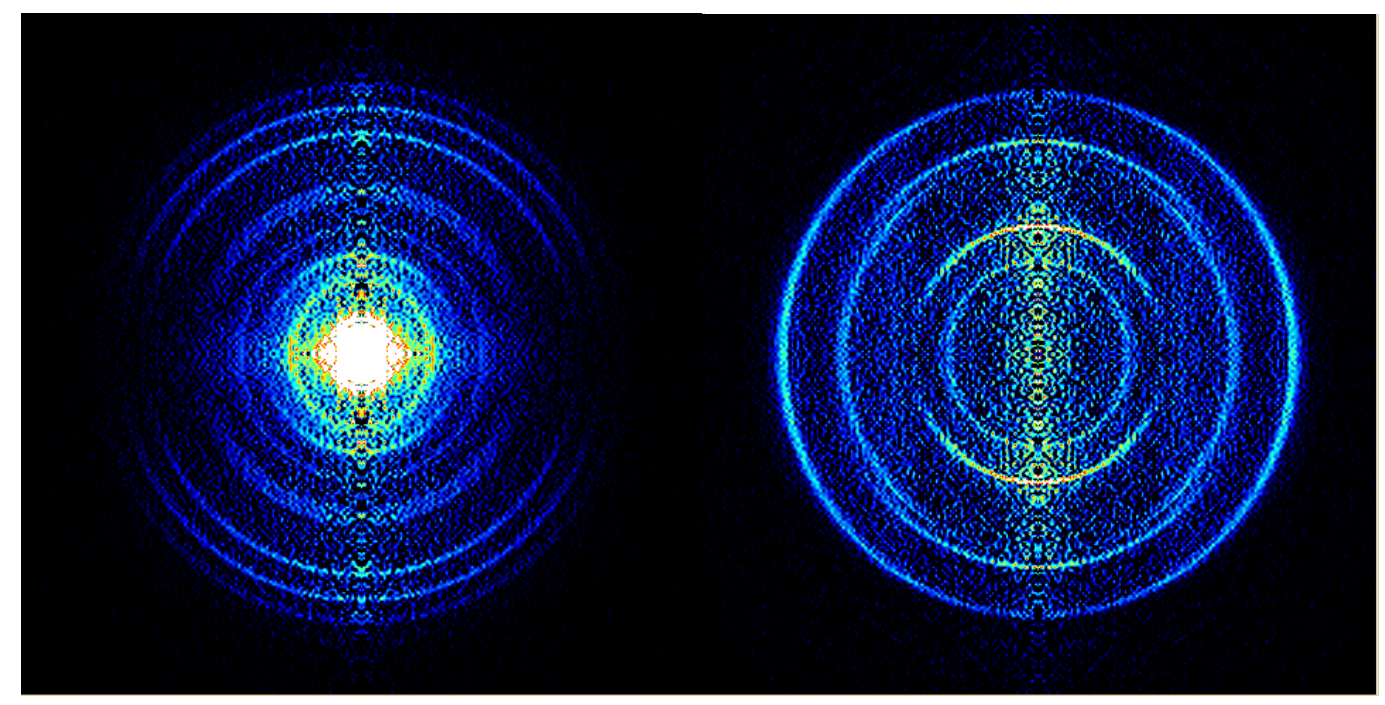

Figure 11.4: Inverted images of $\mathrm{H}^{+}$from $\mathrm{HI}$ dissociation and $\mathrm{I}^{+}$from $\mathrm{I}_{2}$ dissociation processes which were used to calibrate the energy scale of the velocity map imaging. On the left is the $\mathrm{I}^{+}$image, on the right the $\mathrm{H}^{+}$image.

The relation between the speed in pixel and the energy in $\mathrm{eV}$ is given by $\mathrm{R}^{2} / \mathrm{CC}=\mathrm{T}$ with $\mathrm{R}$ the radial distance in pixel, $\mathrm{T}$ the kinetic energy of the particle in $\mathrm{eV}$ and $\mathrm{CC}$ the calibration constant. This relation holds true for 
all masses as long as the voltage settings of the ion lens and the detector stay the same. From the kinetic energy release $(\mathrm{KER}=\mathrm{T})$ of the charged particle, the total kinetic energy release (TKER) can be obtained very easily, since the partner fragments are known. The radial distance is proportional to the radial speed the fragments obtain in the dissociation process.
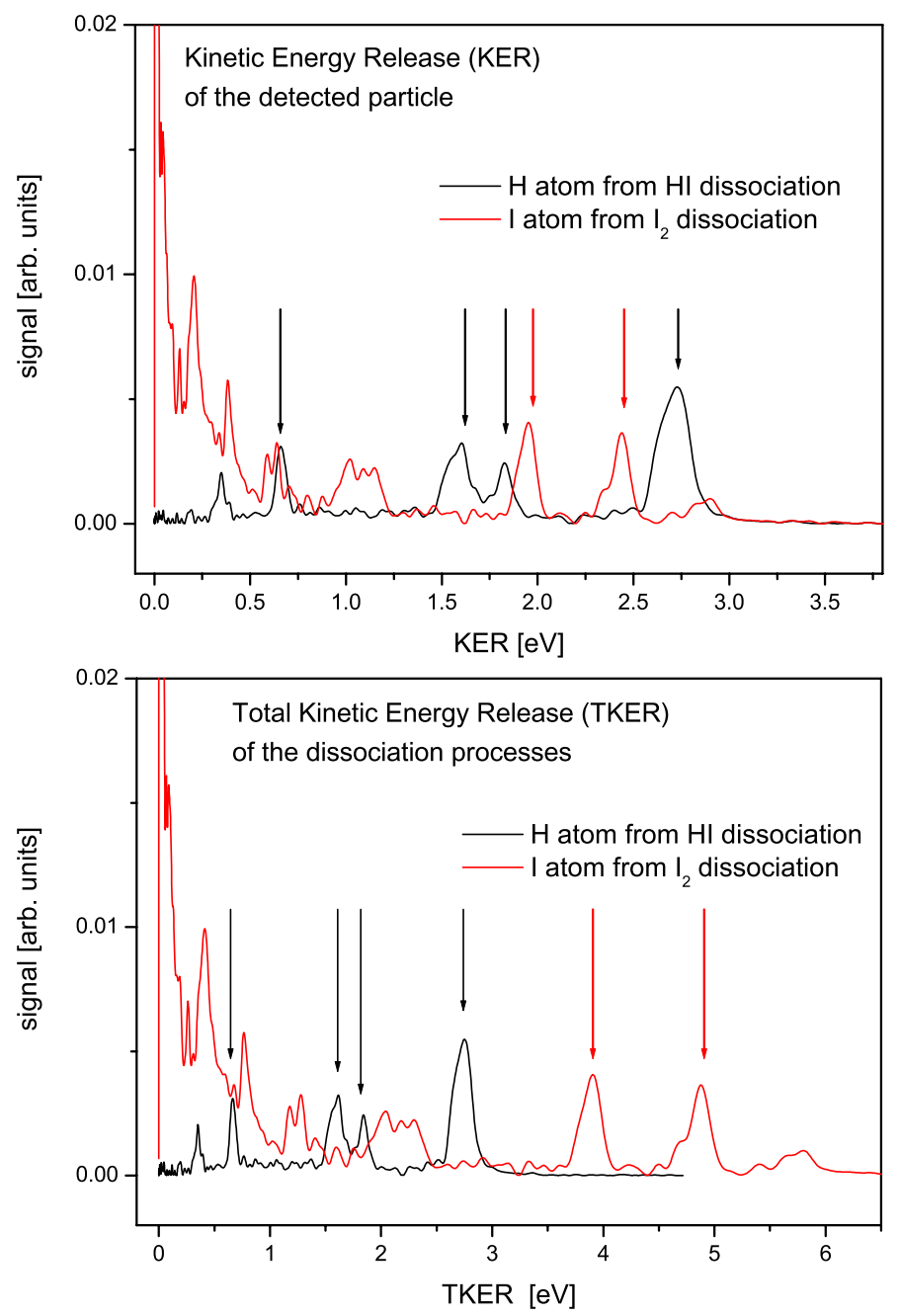

Figure 11.5: The $\mathrm{H}^{+}$and $\mathrm{I}^{+}$distribution depending on the KER of the particle (top figure) and on the TKER of the dissociation process (bottom figure). The peaks used for calibration are indicated with black (HI) and red $\left(I_{2}\right)$ arrows. The small peak at approx $0.36 \mathrm{eV}$ belongs to the dissociation of $\mathrm{HI}$ at $121.53 \mathrm{~nm}$ to $H+I^{*}\left(\left({ }^{3} P_{2}\right) 6 \mathrm{~s}[2]_{5 / 2}, E_{\text {level }}=6.774 \mathrm{eV}\right)$.

Because $\mathrm{HI}$ tends to decay rapidly and form $\mathrm{H}_{2}$ and $\mathrm{I}_{2}$, especially under the influence of daylight, always some $\mathrm{I}_{2}$ is present in an $\mathrm{HI}$ sample. Thus 
the two different species, $\mathrm{H}$ atoms from the $\mathrm{HI}$ dissociation and iodine atoms from the $\mathrm{I}_{2}^{+}$dissociation could be examined by simply picking different masses under exactly the same conditions when using a molecular beam of HI seeded in He. The different masses can be picked by using a gate on the detector because when using velocity map imaging, the different masses are separated along the flight axis by different times of flight (see section 11.1).

Iodine atoms from the HI dissociation were not examined, firstly, because no fitting wavelength was present to ionize the ground state iodine fragment ${ }^{2} \mathrm{P}_{3 / 2}$, secondly, the spin orbit excited fragment ${ }^{2} \mathrm{P}_{1 / 2}$, which may be ionized with $121.53 \mathrm{~nm}$, will have nearly no kinetic energy because the $\mathrm{H}$ atom takes away most of it due to its much smaller mass, and is thus useless for an energy calibration.

The calibration was then done as follows.

- Four different channels were found in the $\mathrm{H}$ images from HI dissociation. With $\mathrm{KER}(\mathrm{H})$ the nominal kinetic energy of the $\mathrm{H}$ atoms and $D_{0}^{0}(\mathrm{HI})=3.054 \mathrm{eV}$, these are

$$
\begin{aligned}
H I+h \nu(212.55 \mathrm{~nm}) & \longrightarrow H+I\left({ }^{2} \mathrm{P}_{3 / 2}\right) \text { with } \operatorname{KER}(\mathrm{H})=2.76 \mathrm{eV} \\
& \longrightarrow H+I^{*}\left({ }^{2} \mathrm{P}_{1 / 2}\right) \text { with } \operatorname{KER}(\mathrm{H})=1.82 \mathrm{eV} \\
H I+h \nu(266 \mathrm{~nm}) & \longrightarrow H+I\left({ }^{2} \mathrm{P}_{3 / 2}\right) \text { with } \operatorname{KER}(\mathrm{H})=1.59 \mathrm{eV} \\
& \longrightarrow H+I^{*}\left({ }^{2} \mathrm{P}_{1 / 2}\right) \text { with } \operatorname{KER}(\mathrm{H})=0.66 \mathrm{eV} .
\end{aligned}
$$

- For all four channels the calibration constant CC was determined. From the four CC values, an average was determined. Ideally, if the imaging operation of the lens is uniform, that constant should not change throughout one image. Here, the fluctuation was less than $1 \%$. The radius in pixel could be determined in minimum steps of $\Delta R=0.25$. Assuming that this is also the maximum error of $\mathrm{R}$, this introduces the maximum error of $\mathrm{CC}$ as a function of $\mathrm{R}$ with

$$
\begin{aligned}
\frac{\Delta C C}{C C} & =\frac{\delta C C(R)}{\delta R} \cdot \frac{\Delta R}{C C} \\
& =\frac{\delta}{\delta R}\left(\frac{R^{2}}{T}\right) \cdot \frac{T}{R^{2}} \cdot \Delta R \\
& =2 \cdot \frac{\Delta R}{R}
\end{aligned}
$$

to be $\Delta \mathrm{CC} / \mathrm{CC}=0.0027 \equiv 0.27 \%$. It follows that the error introduced by the aberrations of the electrostatic lens must be around $0.75 \%$. 
- To calibrate the later $\mathrm{I}^{+}$images now, an $\mathrm{I}^{+}$image which was produced with the same setting as the HI dissociation images. It was evaluated, in the sense that the peak positions in pixel were obtained and then transformed into KER values using the average $\mathrm{CC}$ obtained from the HI images.

- The peak position values with the third and second highest TKER, 3.91 and $4.89 \mathrm{eV}$, were kept and used to calibrate all the other images with different settings and where a $\mathrm{I}_{2} /$ He mixture was used.

These peaks were chosen because firstly, for higher values, the error is usually less, secondly, these rings were among the ones with the smallest width in pixel. The one with the highest TKER, $5.76 \mathrm{eV}$, was not chosen because it has only half the intensity of the those other two and is slightly broader, thus it seemed more error prone than the choice actually made.

- Now from every picture taken under varying conditions, the peak position of these two rings were determined in pixel. These numbers together with the KER from the calibration measurements were then used to calculate a separate $\mathrm{CC}$ for each of the two rings for each image with the relation $\mathrm{R}^{2} / \mathrm{T}=\mathrm{CC}$. Normally, two slightly different $\mathrm{CC}$ values were determined for the two values within one image. Again, the average of these two values was used to determine the final calibration constant for each image.

- Note that although the kinetic energy of the particle $\mathrm{T}=\operatorname{KER}\left(\mathrm{H}^{+}\right)=\operatorname{KER}\left(\mathrm{I}^{+}\right)$is the kinetic energy release of both $\mathrm{H}^{+}$and $\mathrm{I}^{+}$, the total kinetic energy release (TKER) is different. In case of the HI dissociation, the total kinetic energy release is $\operatorname{TKER}(\mathrm{HI})=\frac{\text { mass }_{H} I}{\text { mass }_{I}} \cdot \operatorname{KER}\left(\mathrm{H}^{+}\right)=\frac{128}{127} \cdot \operatorname{KER}\left(\mathrm{H}^{+}\right)$, whereas in case of the $\mathrm{I}_{2}$ dissociation $\operatorname{TKER}\left(\mathrm{I}_{2}\right)=\frac{\text { mass }_{I 2}}{\text { mass }_{I}} \cdot \operatorname{KER}\left(\mathrm{I}^{+}\right)=2 \cdot \operatorname{KER}\left(\mathrm{I}^{+}\right)$is valid. Because in the following chapters where the results of the $\mathrm{I}^{+}$images are discussed always the TKER values are given, here also the TKER values are stated for the $\mathrm{I}_{2}$ dissociation processes. The different scales are illustrated in fig. 11.5.

That the relation $\mathrm{R}^{2} / \mathrm{CC}=\mathrm{T}$ holds true for all masses seems surprising at the beginning. Because they have the same radial distance $\mathrm{R}$ from the centre, we get the impression that both the lighter and heavier mass have the same speed, since the speed obtained in the dissociation process is linearly proportional to the radial distance. Thus it seems that the lighter mass must have a smaller kinetic energy. But because the heavier masses has a 
longer time of flight, the Newton sphere has more time to expand on the way to the detector, which means that two different masses ending up at the same radial distance do not have the same speed, i.e. the factor which relates the speed to the radial distance is mass depending. The time of flight behaves as $t_{T O F} \propto \sqrt{m /(q \cdot U)}$. The expansion velocity obtained from the dissociation is $v_{\text {diss }}=\sqrt{(2 \cdot K E R) / m}$. Because the radial distance behaves as $R \propto v_{\text {diss }} \cdot t_{T O F}=\sqrt{(2 \cdot K E R) / m} \cdot t_{T O F}=\sqrt{(2 \cdot K E R) /(q \cdot U)}$ it follows that the same radial distance on the detector does not correspond to the same speed for different masses, but to the same KER, since the mass is cancelled out as a variable in the radial distance - kinetic energy relation.

\subsection{Determination of the $\beta$-parameter}

To determine the $\beta$-parameter from the 3D-images, one has to keep in mind that the 3D image carries over some of the pixel structure of the 2D-projection. Thus some way of smoothing would be of advantage. To do so, the following procedure was applied.

For each radius $r$, a segment of the width $\Delta r$ is evaluated. This segment is than evaluated for each angle $\Theta$ within a $\Delta \Theta$ by subdividing the segment into smaller units, usually 0.25 pix in radius and $0.25 \mathrm{deg}$ in angle. These units are used to determine an average which is than taken as an intensity value for $r$ and $\Theta$. By choosing $\Delta \Theta$ larger than the steps $\left(\Theta_{\text {step }}\right)$ between the angles $\Theta$, oversampling on overlapping sample areas is achieved and thus an effective smoothing. $\Theta_{\text {step }}$ was chosen as 1 degree, $\Delta \Theta$ as 5 degree and $\Delta r$ as 2 pixel, which is smaller than the FWHM of the narrowest peaks which was at least $\geq 5$ pixel.

This routine is also part of the evaluation routines by André Eppink which can be implemented into the Davis software.

\section{Bibliography}

[1] J.W. Thoman Jr., D.W. Chandler, D.H. Parker, and P.L. Houston. Laser Chem., 9:27, 1988.

[2] A.J.R. Heck and D.W. Chandler. Annu. Rev. Phys. Chem., 46:335, 1995.

[3] D.H. Parker and A.T.J.B. Eppink. Jour. Chem. Phys., 107:2357, 1997.

[4] A.T.J.B. Eppink and D.H. Parker. Review of Scientific Instruments, 68:347, 1997. 
[5] R.N. Bracewell. The Fourier Transform and its Applications. McGrawHill, New York, 1986.

[6] L.M. Smith and D.R. Keefer. J. Quat. Spectrosc. Radiat. Transfer, 39:367, 1988.

[7] Y. Sato, Y. Matsumi, M. Kawasaki, K. Tsukiyama, and R. Bersohn. Jour. Phys. Chem., 99:16307, 1995.

[8] C. Bordas, F. Pauling, h. Helm, and D.L. Huestis. Review of Scientific Instruments, 67:2257, 1996.

[9] J. Winterhalter, D. Maier, J. Honerkamp, V. Schyja, and H. Helm. Jour. Chem. Phys., 110:11187, 1999.

[10] H.P. Loock and S. Manzhos. Computer Physics Communications, 154:76, 2003.

[11] M.J.J. Vrakking. Review of Scientific Instruments, 72:4084, 2001.

[12] A. Eppink, S.M. Wu, and J.B.C. Whitaker. Reconstruction methods. In J.B.C. Whitaker, editor, Imaging in Molecular Dynamics: Technology and Applications, chapter 3, page 65. Cambridge University Press, 2003.

[13] V. Dribinski, A. Ossadtchi, V.A. Mandelshtam, and H. Reisler. Review of Scientific Instruments, 73:2634, 2002. 


\section{Chapter 12}

\section{Experimental Setup}

\subsection{Detector and ion lens}

For the VMI experiments, the original vacuum chamber as described in chapter 3 was fitted with an ion lens, which was mounted directly onto the plate holding the skimmer for the molecular beam.

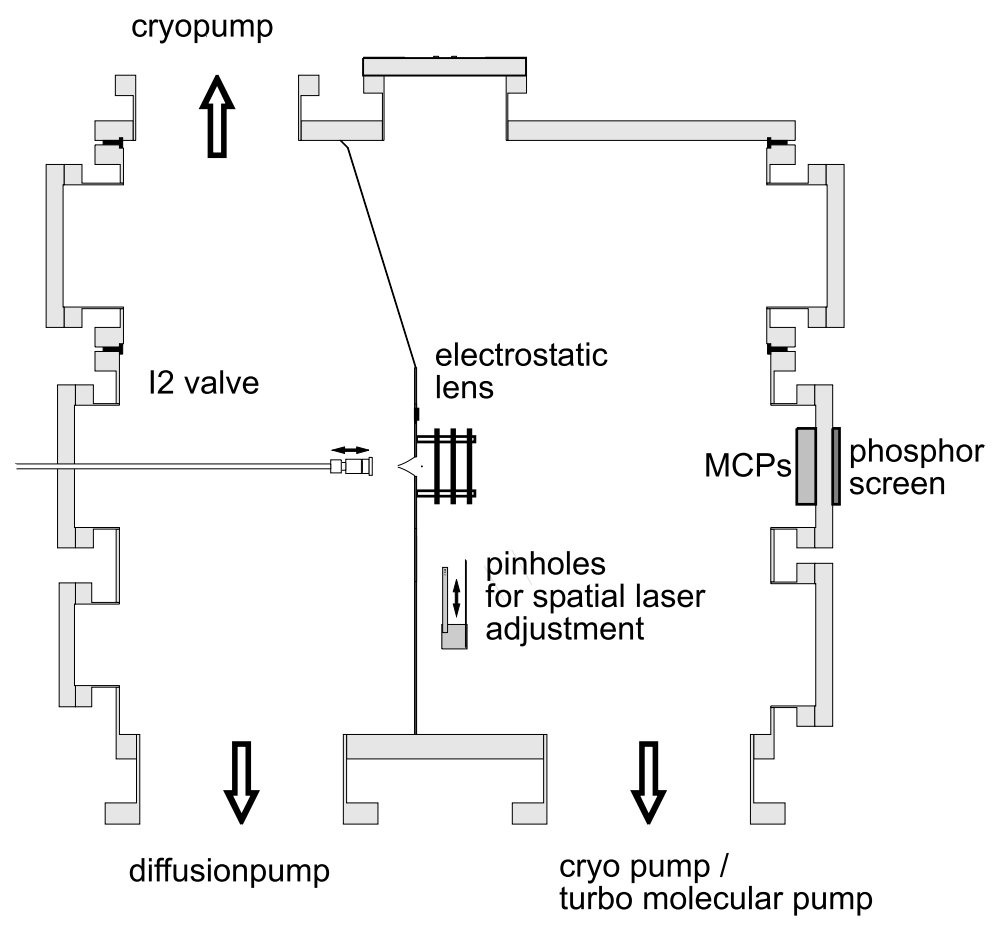

Figure 12.1: Vertical cut through the vacuum chamber with the velocity map imaging set-up. 
The voltages on the ion lens were normally $+3 \mathrm{KV}$ on the repeller and around $+2.1-+2.2 \mathrm{KV}$ on the extractor plate. When measuring negative particles, the voltages on the lens were simply set as negative voltages of the same value.

The drift path to the detector was not particularly shielded, the walls of the chamber were as far away as $40 \mathrm{~cm}$.

The detector consisted of two micro channel plates (MCP) (manu: Proxitronic, $75 \mathrm{~mm}$ active diameter, $1.5 \mathrm{~mm}$ thick, $25 \mu \mathrm{m}$ pores, $\mathrm{L} / \mathrm{D}=60 / 1$, medium rate) and a $\mathrm{P} 43$ phosphorscreen (manu: Proxitronic). The MCP were stacked with a ring of $0.2 \mathrm{~mm}$ in between which allowed to operate the front $\mathrm{MCP}$ separately. Because in VMI different masses are effectively separated by the time of flight, the detector was only set active for the appropriate mass. Typical voltage values were $4.5 \mathrm{KV}$ on the phosphorscreen, $1.2 \mathrm{KV}$ on the exit side of the back MCP, $300 \mathrm{~V}$ on the electrode ring connecting them, and $-600 \mathrm{~V}$ on the entry side of the front plate when the detector was active and $0 \mathrm{~V}$ when it was inactive. This has the advantage that the voltage has not to build up over the whole stack of MCPs, but only the voltage on the entry side of th front plate has to be changed. The setting was as such that the front plate was set to a voltage just not sufficient to achieve amplification when inactive, which allows the back plate to be still active.

For changing the voltage on the entry side a high voltage switch (GHTS 100, manu: Behlke) was used. The high voltage switch, camera and computer were synchronised with a digital delay generator (DG535, manu: Stanford Research Systems, Inc.) which also controlled the length of the gate. The gate was usually set to nominally $300 \mathrm{~ns}$, but with the rise time of the switch typically in between 10 and $100 \mathrm{~ns}$, depending on the capacity of the object, the real active time is notedly smaller.

With the above setting, the iodine mass started at $7.8 \mu \mathrm{s}$ time of flight.

The voltages on the detector for negative particles (electrons, $\mathrm{I}^{-}$) were $1.8,0.9$ and $0 \mathrm{KV}$, to set the detector inactive, the $0 \mathrm{KV}$ voltage on the entry side was changed to $300 \mathrm{~V}$.

Because no servo motors were used as in the Nijmegen set-up, which allow to align the lens during the experiment, the whole skimmer plate had to be slightly tilted in several steps with the chamber brought up to air to ensure that the image was centred on the MCP. The final set-up after this trial-anderror procedure was kept throughout the experiments, small deviations due to thermal expansions did not cause grave problems because of the relatively large diameter of the plates. The images were usually $40-50 \mathrm{~mm}$ in diameter, whereas the MCP had a diameter of $75 \mathrm{~mm}$.

The camera used was a PixelFly (manu: PCO, with a VGA CCD sensor, $640 \times 480$ pixels), for the camera control and image recording, DaVis software 
(manu: LaVision) was used, in which the event counting routine could be implemented.

\subsection{The $\mathrm{I}_{2}$ molecular beam}

The seeded molecular beam of $\mathrm{I}_{2}$ in He is produced by expanding the gas into the vacuum via a pulsed valve and then skimming the resulting beam (valve: General Valves, series no. 9-500-900, orifice diameter 0.5 mm; skimmer: Beam Dynamics, model 2, skimmer orifice $2.1 \mathrm{~mm}$, height $26.65 \mathrm{~mm}$. The valve was on axis with the central axis of the detector. The background pressure in the nozzle chamber with the nozzle on was $\approx 2 \cdot 10^{-5}$ mbar.

The iodine was used at room temperature (vapour pressure $\approx 0.5 \mathrm{mbar}$ ), with helium as a seed gas with a total stagnation pressure of 1-2 bar. Iodine with $99.8 \%$ purity (manu: Riedel de Haen) was used. Using a seed gas gives a better expansion and thus a more effective cooling of the internal degrees of freedom of the iodine.

The iodine was used without further purification, after loading the gas system, the system was shortly pumped down and then filled with the seed gas. The sample was then shortly warmed up to a higher pressure, to achieve quick covering of the surfaces with $\mathrm{I}_{2}$ and thus a uniform vapour pressure in the relatively long supply line and the nozzle.

Miller [1], who determined the vibrational levels in the initial beam via multiphoton ionization, found $v \longleftarrow 0$ and $v \longleftarrow 1$ bands in his spectra. He used similar expansion conditions as in this experiment (iodine vapour of $\approx 0.7$ mbar and a total backing pressure of $1-5$ bar, seeded in Helium or Argon). He compared the vibrational distribution with one taken from a cell experiment and found a considerably colder distribution, which is fairly well described with $\mathrm{T}_{v i b} \approx 150 \mathrm{~K}$. Additionally, Miller and Compton [2, 3] and McClelland et al. [4] reported a rotational temperature of $\mathrm{T}_{\text {rot }} \approx 10 \mathrm{~K}$ for similar conditions.

To pinpoint any results due to I-rare gas clusters, some experiments were performed with a different seed gas, i.e. argon. Although $\mathrm{I}_{2}$-Ar clusters were present in the mass spectrum, no different results were obtained in the dissociation experiments, apart from a slightly slower velocity obtained in the expansion. Thus it can be concluded that clustering did not contribute to the results of the dissociation experiments.

The temporal and spatial profile of the molecular beam pulse were measured by a fast ionization gauge (manu: Beam Dynamics, type: FIG-1, FH-1 gauge head) which is attached to a movable mount within the chamber and therefore can be moved into the beams. Distance between gauge head and 
probe laser axis was then $217 \mathrm{~mm}$. The gauge was controlled by the driver unit (manu: Beam Dynamics, type FIG-1, FC-1 control unit). The response risetime of the gauge is $3 \mu \mathrm{s}$, together with its small detection volume,the pulses could be temporally and spatially resolved, typical pulse lengths were $1 \mathrm{~ms}$.

The extreme broadness of the pulse is probably enhanced because the pulse is disturbed downstream of the skimmer by the repeller plate. It has only an opening of $2 \mathrm{~mm}$ in diameter, whereas the molecular beam has a typical spatial FWHM (full width at half maximum) of $5 \mathrm{~mm}$. This may disrupt the flow, lengthen the pulse and dampen its intensity. Because the gauge was not calibrated in a $\mathrm{He} / \mathrm{I}_{2}$ atmosphere, no absolute figures for the particle density of the pulses can be given.

\section{Bibliography}

[1] J.C. Miller. Jour. Phys. Chem., 91:2589, 1987.

[2] J.C. Miller and R.N. Compton. Jour. Chem. Phys., 75:22, 1981.

[3] J.C. Miller and R.N. Compton. Jour. Phys. Chem., 75:2020, 1981.

[4] G.M. McClelland, K.L. Saenger, J.J. Valentini, and D.R. Herschbach. Jour. Phys. Chem., 83:947, 1979. 


\section{Chapter 13}

\section{Mass Spectrum and Photoelectrons}

Since this work deals with the photofragmentation of $\mathrm{I}_{2}$ mainly at photon energies above the first ionization threshold, photoionization is of course a major process. Thus a photoelectron image was taken to determine the $\mathrm{I}_{2}^{+}$ states achieved with this photon energies, and mass spectra to obtain the ratio between $\mathrm{I}^{+}$and $\mathrm{I}_{2}^{+}$.

\subsection{Mass spectrum}

The mass spectra were taken in two different ways. In both cases laser power and intensity of the molecular beam were comparable to the conditions for the $\mathrm{I}^{+}$images.

For an overall mass spectrum, the detector was set permanently active, i.e. there was no gate in which the MCP detector was only active to select the appropriate mass. The phosphor screen was then used as a detector of the total ion signal and the ion signal was separated from the high voltage (HV) applied to the phosphor screen via a capacitor. Since the different masses got different flight times, the time resolved mass spectrum could subsequently be displayed and recorded with a digital oscilloscope. The obtained TOF spectrum was then converted to a mass scale. This classical mass spectrum was useful to determine the ratio of $\mathrm{I}^{+}$to $\mathrm{I}_{2}^{+}$and to detect signals from impurities with different masses with a significant intensity.

To detect masses with a low intensity, which would not show clearly in a classical mass spectrum like above, a manual scan was performed. The MCP gate of $300 \mathrm{~ns}$ (see chapter 11) was shifted in steps of $100 \mathrm{~ns}$ from the first signal $\left(\mathrm{t}_{\mathrm{TOF}}=0 \mu \mathrm{s}\right)$ induced by scattered UV and VUV light until no 
further signal $\left(\mathrm{t}_{\mathrm{TOF}}>16.1 \mu \mathrm{s}\right)$ appeared. Because here the imaging detector was used, not only masses which only gave sporadic ions could be detected, but also it could be discerned if the ions showed a spatial distribution or were only an unstructured 'blob' in the middle of the image.

In the former case, the ions were produced in a dissociative process which would give structured rings, in the latter case the ions were produced by ionization of the initial atom or molecule, where the kinetic energy is taken away by the electron, and thus the ions will stay on the middle axis and form a 'blob' in the middle of the image.

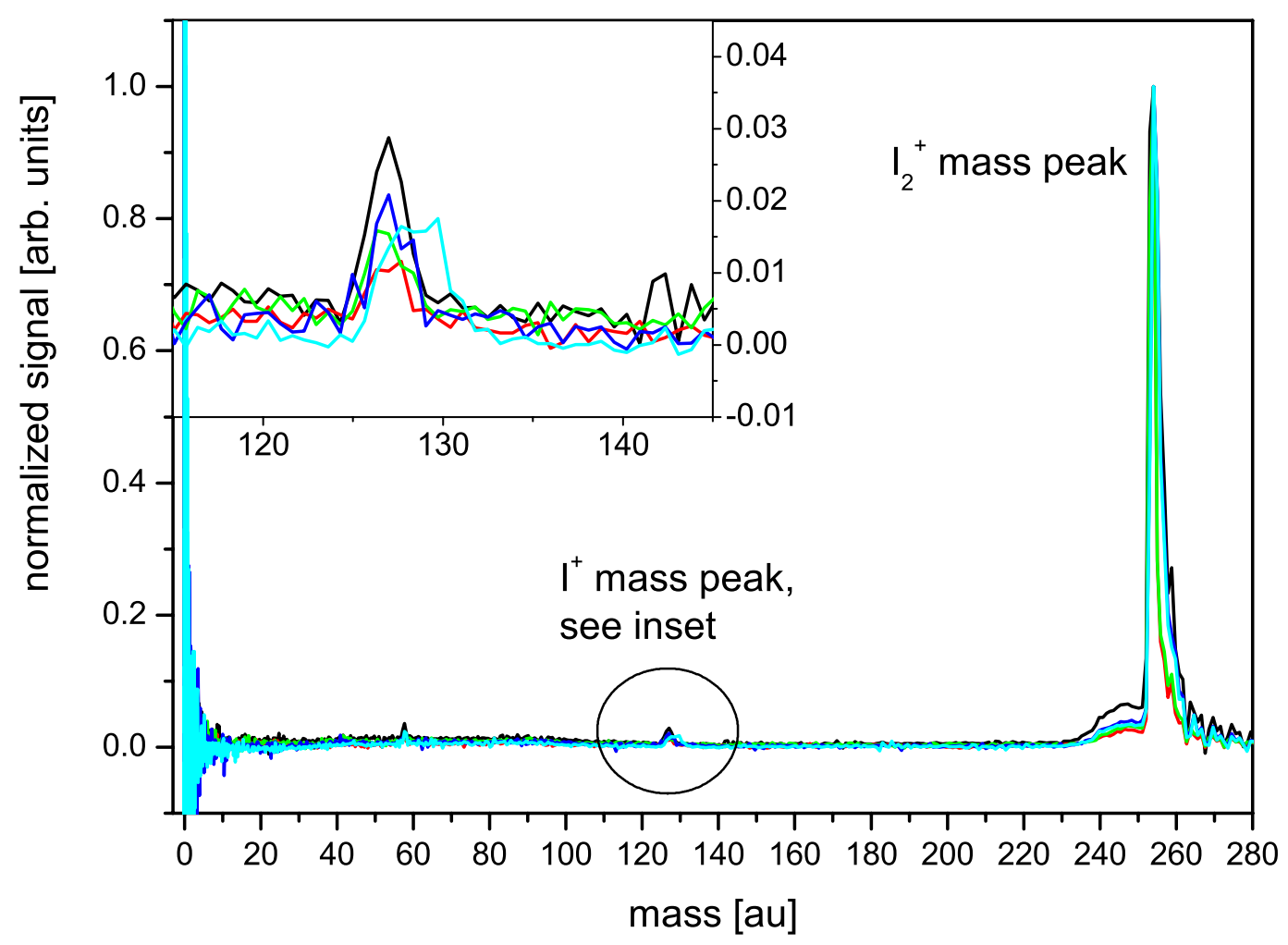

Figure 13.1: Mass spectrum. The I mass peak at 127 au is shown in the inset.

The overall mass spectrum is shown in fig. 13.1. The spectrum reveals that about 70 times more $\mathrm{I}_{2}^{+}$is produced compared to $\mathrm{I}^{+}$, therefore most of the electrons observed here belong to $\mathrm{I}_{2}^{+}$production.

No evidence of clustering was found, only $\mathrm{I}$ and $\mathrm{I}_{2}$ mass peaks were present. As well no significant $\mathrm{Br}$ or $\mathrm{Br}_{2}$ mass peaks were found, which was especially checked, since $\mathrm{I}_{2}$ frequently contains traces of $\mathrm{Br}_{2}$ and with that of $\mathrm{IBr}$, which could interfere with the results by contributing $\mathrm{I}_{\text {or }} \mathrm{I}^{+}$ from a dissociative process. 
The only other mass found in a concentration similar to iodine is mass 58 , which is a typical indication for pump oil contamination, possibly diffusion pump oil (Santovac 5). But because of its different mass, it does not influence the $\mathrm{I}^{+}$images. Electrons from the ionization of the pump oil will of course be present, but since it is originally a five ring polyphenyl ether, it will possibly not result in a peaked contribution to the photoelectron images, but could account for the amount of underlying electrons in the image, due to its high number of electrons and degrees of freedom.

One other aspect of the mass spectrum is that no significant difference for the different wavelengths combinations (VUV, VUV+365 nm or $\mathrm{VUV}+266 \mathrm{~nm}$ ) occurred, in the sense that the $\mathrm{I}_{2}^{+} / \mathrm{I}^{+}$ratio changed dramatically or that different masses showed up in the spectrum.

The manual scan of the masses as described above, showed faint unstructured blobs in the middle of the image at $\mathrm{Br}$ and $\mathrm{Br}_{2}$ mass, which means that both are produced from direct ionization, but not in a dissociative process, where the $\mathrm{Br}$ or $\mathrm{Br}^{+}$should take away kinetic energy. Apart from those already mentioned, other masses (e.g. typical masses as 44 from $\mathrm{CO}_{2}$ ) were only present as faint dots or blobs in the image centre or as sporadic ions.

\subsection{Photoelectron images}

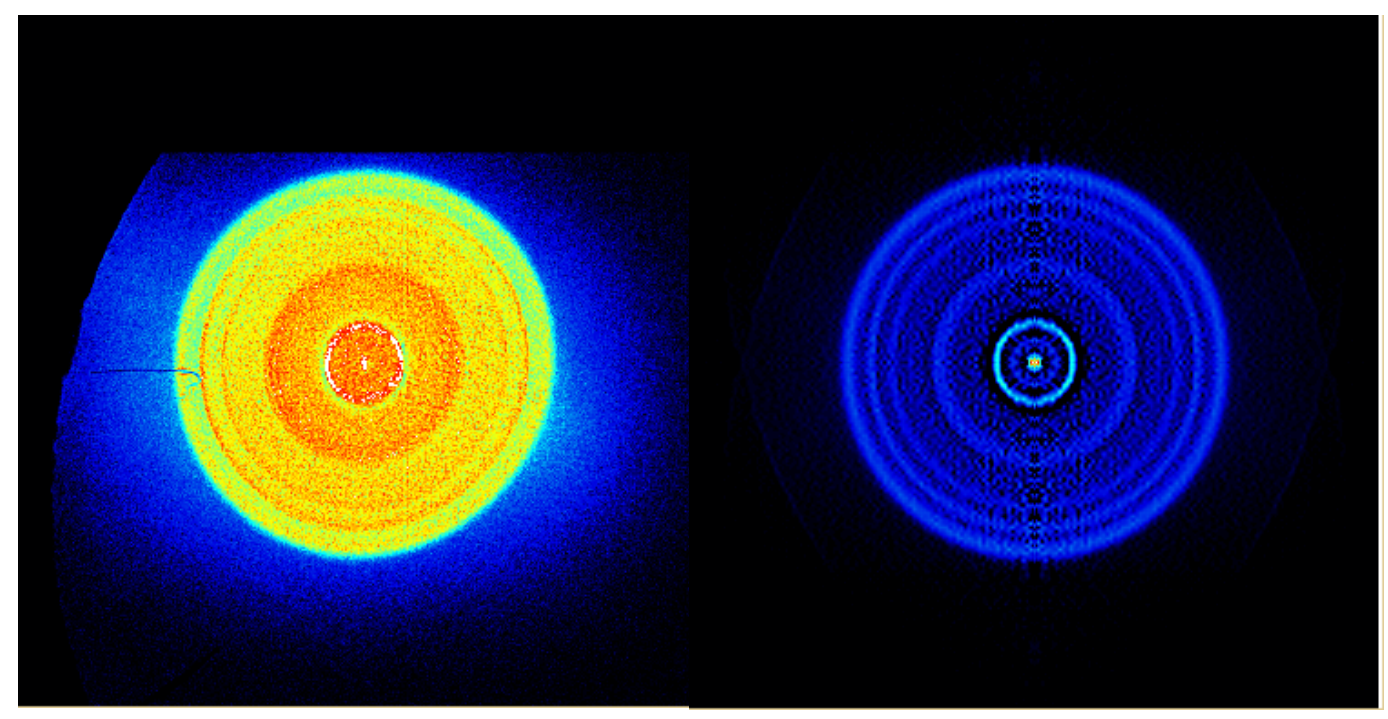

Figure 13.2: Raw and inverted image of photoelectrons with VUV

The raw and converted images are shown in fig. 13.2. The images were taken with a $300 \mathrm{~ns}$ gate starting at $200 \mathrm{~ns}$ after the VUV laser pulse. Five 
rings can be clearly made out. The second (counted from inside to outside) one and the outmost one are rather broad, the innermost one is the sharpest. In the raw image it is still visible that a big unstructured cloud of electrons underlies the structured signal.

Because the electrons are easily deflected by stray fields, external permanent magnets were attached to the chamber to compensate. Still, it was not possible to centre the image exactly onto the detector, which can be made out in the raw image, where the outline of the detector is visible to the left. Due to the fields, the 'rings' are no perfect circles but slightly deformed. The deconvolution algorithm symmetrizes the pictures by mirroring them along the vertical and horizontal axis, thus the rings in the converted image may appear slightly broader than they really are.

Additionally, the image is unfortunately not focused in all places. A radial axis through the images exists which exhibits a splitting of the third ring into actually three vibrational states, but because of the mirroring by the algorithm, this disappears in the inverted image and has to be taken into account separately.

Since for negative charged particles to be measured the detector as well as the ion lens had to be operated differently. For the ion lens, the voltages used for positive ions were only set to negative voltages of the same value, but for the detector, things are more complicated. Here, the front plate could not be put on a negative voltage any more, but still the overall potential gradient along the MCP assembly should stay the same. Therefore the MCP voltages were slightly altered, as described in chapter 12.1. Because especially the setting of the front MCP was altered, this causes unfortunately that the scaling of the rings changes slightly.

To obtain the a scaling for the photoelectron images, I used results of a photoelectron spectrum measured under similar conditions. Higginson et al. [1] measured a high resolution photoelectron spectrum of the $\mathrm{X}^{2} \Pi_{g}$ states of $\mathrm{I}_{2}^{+}$. They used NeI radiation (16.67-16.85 eV) to ionize the iodine molecule. Although they used a a different wavelength with a higher photon energy, the results should be comparable. Firstly, they used a one photon transition, which also is effective here for the $\mathrm{X}^{2} \Pi_{g}$ states, and secondly, because the difference in bond length of the neutral $\mathrm{X}^{1} \Sigma\left(0_{g}^{+}\right)$and the ionic $\mathrm{X}^{2} \Pi_{g}$ ground state of the iodine molecule is very small, the higher photon energy will probably not cause an extremely high vibrational excitation. Actually, the authors only found low vibrational states, peaking at $\mathrm{v}^{\prime}=1$ for both spin orbit components, with mainly four vibrational quanta populated. Miller [2] performed multi photon ionization in a molecular beam, and although he 
found up to six vibrational quanta this way, he also found $\mathrm{v}^{\prime}=0$ and $\mathrm{v}^{\prime}=1$ to be the most populated vibrational states.

In the TKER distribution of the photoelectrons measured here (fig. 13.3), three vibrational quanta were clearly visible for the $\mathrm{X}^{2} \Pi_{1 / 2, g}$ state, especially in the raw image, where the first and the second state were approximately equally populated. In the spectrum by Higginson et al. the v'=1 peak has the highest intensity, with decreasing intensity of the higher vibrational quanta, thus it is conceivable to assign the first peak found here to v'=1. But because their $\mathrm{v}^{\prime}=0$ peak is still slightly higher than the $\mathrm{v}^{\prime}=2$ peak and the $\mathrm{v}^{\prime}=3$ peak shows a considerably smaller population, I assign the two peaks of equal intensity found here to $v^{\prime}=0$ and $v^{\prime}=1$ as indicated in TKER distribution shown in fig. 13.3. The nominal TKER values of these two peaks $(9.952 \mathrm{eV}$ and $9.980 \mathrm{eV})$ were then used for the energy scaling of the entire kinetic energy distribution.

For ionization in a field, lowering of the ionization energy by the subtraction field has to be taken into account. The modification of the transition energy is given by $\Delta \mathrm{E} \approx 4 \times \sqrt{\mathrm{V} \text { per } \mathrm{cm}}$. This gives for the field of the imaging lens of $800 \mathrm{~V}$ over a distance of $3 \mathrm{~cm}$ a lowering of roughly $0.008 \mathrm{eV}$ [3]. All resulting transition energies given are corrected for this value.

When measuring the photoelectrons, I had to reduce the VUV power immensely (full set of plates) to obtain distinguishable events so that the event counting algorithm could be used. Since the mass spectrum reveals (see fig.13.1) that about 70 times more $\mathrm{I}_{2}^{+}$is produced compared to $\mathrm{I}^{+}$, most of the electrons observed here belong to $\mathrm{I}_{2}^{+}$production. Any electrons originating from $\mathrm{I}^{+}$production other than $\mathrm{I}_{2}^{+}$dissociation will quite possibly go unnoticed, since they are to few to form rings and so be recognisable as a separate process. A ring in the photoelectron spectrum from $\mathrm{I}_{2}^{+}$production has an average signal of 150 counts. This would mean that a ring from a process other than that would have an average of 2 counts. Rings like that can be faintly made out in an area where no other rings lie (see traces of VUV rings in VUV+266 nm images), but will probably be indistinguishable from the intensity fluctuations of the photoelectron image.

Also some thought should be given to the undefined blob of electrons underlying the structured image. Because of the gate set to the detector, anything else than electrons can be excluded as possible causes of the signal. As explained above, electrons from any other state, for example dissociative ionic states which will give a rather broad distribution of electrons, can be also excluded because the corresponding $\mathrm{I}^{+}$were only observed in much lower 
number in the $\mathrm{I}^{+}$images. Since this blob is not round which it should be if it was produced in the dissociation volume within the electrostatic lens or close nearby, but is still located near the proper electron image, I assume that these stray electrons are produced by the VUV laser from gas traces within the whole volume of the electrostatic lens. Since oil pump was found in the mass spectrum it may be also partly responsible for these stray electrons.

\subsection{1 $\quad \mathrm{X}^{2} \Pi_{g}$ states}

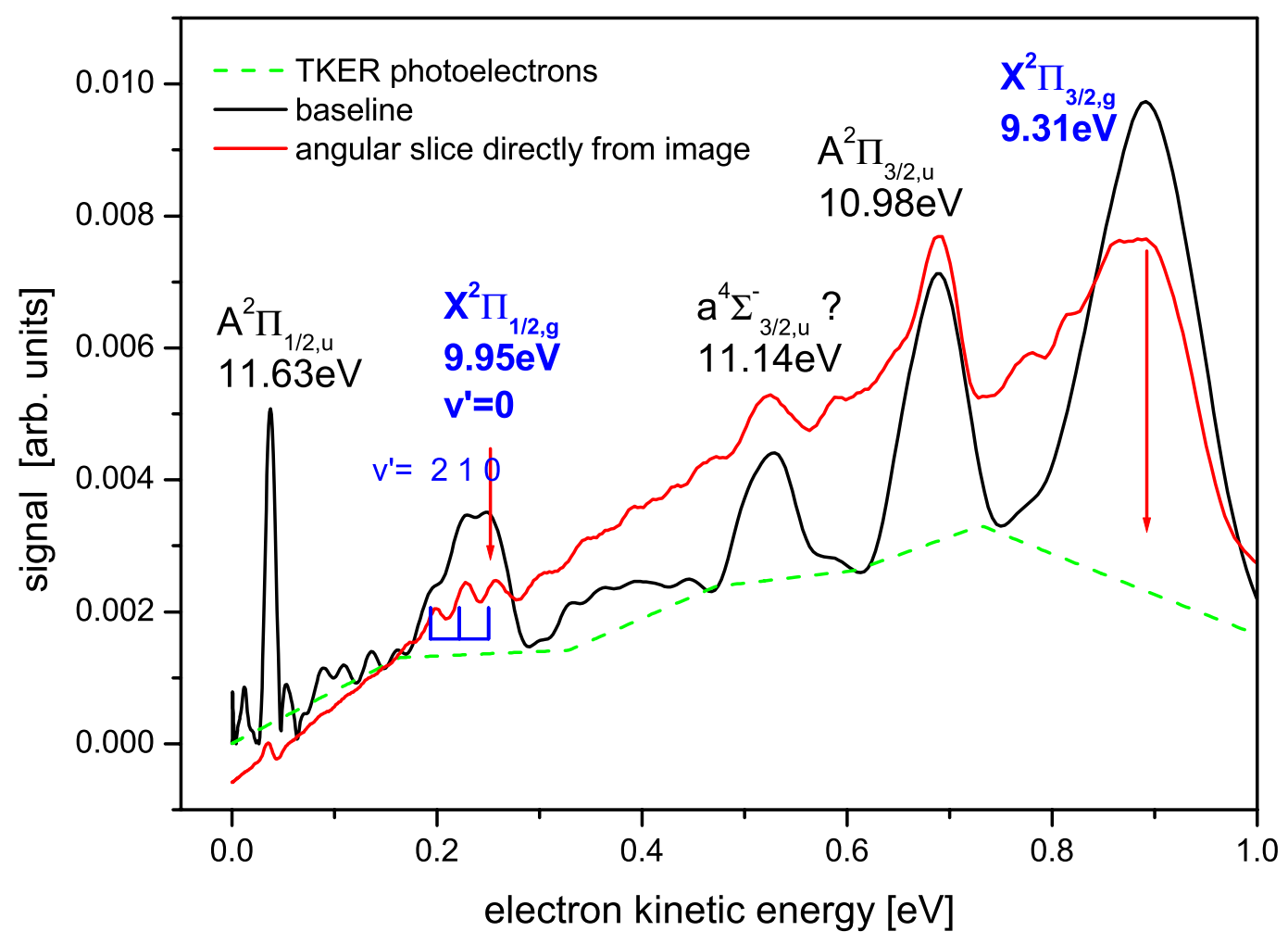

Figure 13.3: Kinetic energy distribution of the photoelectrons from the $I_{2}^{+}$ production. The black line is from a converted image, see text. The energy scale is the measured TKER corrected for the applied electric field. Blue numbers correspond to ionization by one VUV photon $(10.20 \mathrm{eV})$ and the black numbers to processes with two $212.55 \mathrm{~nm}$ photons $(11.67 \mathrm{eV})$. The red arrows indicate the vertical transition energy of the $X^{2} \Pi_{g}$ states. The red line is an angular slice taken directly from the raw image. Only the $\mathrm{x}$ axis was converted from pixels to kinetic energy. The green line represents the base line, above of which the intensity was determined, see text. 
The kinetic energy distribution is shown in figure 13.3. The black line is taken from the converted image which corresponds to the cut through the $3 \mathrm{D}$ distribution. The red line is an angular slice through the raw image (2Dprojection) at the angle where the focus is best. The peaks are slightly altered in shape with respect to the converted picture, which is to be expected. But since the vibrational levels stretch only over a small energy range, the different shape does not make much of a difference and the determined energy gaps should represent real energy gaps.

The experimental data from this work in comparison with older experimental data are summarised in table 13.1. Here, relative intensities of the peaks were also given. These were determined for the peaks above the baseline (dashed green line in figure 13.2). These are therefore only a rough estimate, but the baseline takes into account the undefined blob of electrons which shows on the side of the real projection in the raw image of the photoelectrons.

Note that only comparisons of intensities of processes with the same excitation energy are meaningful.

\begin{tabular}{|l||c|c|c|c|c|}
\hline $\mathrm{I}_{2}^{+}$ & $\mathrm{X}\left({ }^{2} \Pi_{3 / 2, g}\right)$ & $\mathrm{X}\left({ }^{2} \Pi_{1 / 2, g}\right)$ & $\mathrm{A}^{2} \Pi_{3 / 2, u}$ & $\mathrm{~A}^{2} \Pi_{1 / 2, u}$ & $\mathrm{a}^{4} \Sigma_{3 / 2, u}^{-} ?$ \\
\hline \hline exp. transition energy, & 9.31 & 9.95 & 10.98 & 11.63 & 11.14 \\
this work [eV] & & & & & \\
\hline normalised intensity [a.u.] & 0.61 & 0.10 & 0.17 & 0.08 & 0.03 \\
\hline excitation energy [eV] & 10.20 & 10.20 & 11.67 & 11.67 & 11.67 \\
no. photons & 1 & 1 & 2 & 2 & 2 \\
\hline$\beta$-parameter & 0.0 & -0.1 & -0.07 & -0.22 & -0.08 \\
$\pm \Delta \beta \approx$ & 0.05 & 0.08 & 0.05 & 0.1 & 0.07 \\
\hline exp. vertical transition & 9.307 & 9.952 & 10.71 & 11.54 & 10.66 \\
energy, literature $[\mathrm{eV}]$ & & & -11.03 & -11.82 & \\
\hline
\end{tabular}

Table 13.1: Experimental results of the photoelectron image. Results of this work are shown, together with their relative intensity, their excitation energy and their $\beta$-parameter. Experimental transition energy of this work are given at the highest intensity. Note that only a comparison of intensity of processes with the same excitation energy are meaningful. Experimental results found in literature are given in the last line.

The ionization channel which gives the highest TKER in the measured image was identified as a transition to $\mathrm{I}_{2}^{+}\left(\mathrm{X}^{2} \Pi_{3 / 2, g}\right)$ ground state, peaked at a transition energy of $9.31 \mathrm{eV}$. This agrees fairly well with latest vertical ionization energy of this state, determined by Cockett et al [4], which 
is $9.307 \mathrm{eV}$. The outmost ring corresponding to this state does only show a broadening, which hints at a vibrational distribution, but the resolution is not good enough to give separate rings. From the slice through the 2D raw image, one can suspect that four vibrational quanta are present, with $\mathrm{v}^{\prime}=0$ and $\mathrm{v}^{\prime}=1$ equally populated.

The state to which the vibrational distribution around $0.2 \mathrm{eV}$ belongs to is the spin orbit excited component of the ground state $\mathrm{I}_{2}^{+}\left(\mathrm{X}^{2} \Pi_{1 / 2, g}\right)$, the value obtained here for the vibrational constant is $\omega=0.029 \mathrm{eV}$, which agrees well with the spectroscopically determined value of $0.0284 \mathrm{eV}$ for this state [4]. Three vibrational levels can be clearly made out, with decreasing intensity for smaller energy, which indicates that these are vibrational levels of the product, not the initial beam. If the vibrational spacing would come from initial excitation, it is to be expected that the population of the vibrational levels decreases with growing energy, i.e. the higher the vibrational level in the initial beam, the smaller the population. As a cautionary remark, one should keep in mind that the vibrational spacing of the neutral iodine ground state is only slightly smaller with $\omega=0.0265 \mathrm{eV}[5,6]$. Therefore it is impossible to judge only from the $\omega$-value if they belong to neutral or the ion. But as was mentioned in section 12.2 Miller [2] could only find $v \longleftarrow 0$ and $v \longleftarrow 1$ bands in his spectra in his multiphoton spectra of $\mathrm{I}_{2}^{+}$from a molecular beam under similar conditions. Because here higher vibrational quanta were found, it supports the assumption that the vibrational distribution belongs to the ion $\mathrm{I}_{2}^{+}$and not to the neutral molecule $\mathrm{I}_{2}$.

Still the assignment of the peaks is a tentative one. As mentioned, in fig. 13.3, the first clear peak is assigned to $\mathrm{v}^{\prime}=0$ with a ionization energy of $9.952 \mathrm{eV}$ [4], based on the results of Miller [2] and Higginson et al. [1]. But their results would also allow to assign the first clear peak on the high TKER side to $\mathrm{v}^{\prime}=1$ instead of $\mathrm{v}^{\prime}=0$. Thus this uncertainty remains.

The vibrational excitation is quite low because in the ionization to $\mathrm{X}^{2} \Pi_{g}$ state, a non-bonding $\pi$-electron is removed and the bond length changes only slightly from $\mathrm{r}_{e}=2.66 \AA$ of the molecular ground state of the neutral $\mathrm{I}_{2}\left(\mathrm{X}^{1} \Sigma\left(0_{g}^{+}\right)\right)(2440)$ to $\mathrm{r}_{e}=2.58 \AA$ for the ionic ground state $\mathrm{X}^{2} \Pi_{g}(2430)$. The orbital configuration of the valence electrons of the molecular iodine states are given in the notation by Mulliken [7] in klmn meaning $\sigma_{g}^{k} \pi_{u}^{l} \pi_{g}^{* m} \sigma_{u}^{* n}$.

\subsection{2 $\quad \mathrm{A}^{2} \Pi_{u}$ states}

Because only the ground state is accessible with one VUV photon, all other channels must belong to multiphoton processes, most likely to two UV photons, which gives a total energy of $\mathrm{E}_{h \nu}=11.67 \mathrm{eV}$. The ionization to the first 
excited state, $\mathrm{A}^{2} \Pi_{3 / 2, u}$ and $\mathrm{A}^{2} \Pi_{1 / 2, u}$ can then be identified as the innermost and the fourth peak from inside out.

The ionization values in literature for the $\mathrm{A}^{2} \Pi_{u}$ (2340) states differ more than the ones for the $\mathrm{X}^{2} \Pi_{g}$ state. This is probably due to the fact that the bond length changes more for transitions from the molecular ground state $\mathrm{X}^{1} \Sigma\left(0_{g}^{+}\right)\left(\mathrm{r}_{e}=2.66 \AA\right)$ to the $\mathrm{A}^{2} \Pi_{u}\left(\mathrm{r}_{e}=2.99 \AA\right)$ states states than to the $\mathrm{X}^{2} \Pi_{g}\left(\mathrm{r}_{e}=2.58 \AA\right)$ states, because of the removal of a bonding $\pi$-electron. This may introduce a greater uncertainty depending on the experimental method. The most recent ionization energy values in literature values are by Cockett et al. [4, 8] and by Yencha et al. [9], which were obtained by threshold photoelectron spectroscopy. Most older data were obtained from electron collision ionization or conventional photoelectron spectroscopy measuring excess kinetic energy, they either give vertical transition and/or adiabatic energy values.

The transition energy to the first electronically excited states range in between $10.708 \mathrm{eV}$ and $11.03 \mathrm{eV}$ for the lower component and in between 11.54 and 11.82 for the higher component. Here, the transition energies were determined as 10.98 and $11.63 \mathrm{eV}$, which is in good agreement with known experimental results. Yencha et al. [9] achieved vibrational resolution in their spectra, but could not clearly identify a band head, thus their assignments give only an approximate values. The results here for maximum intensity correspond to their peak no. 20 for $\mathrm{A}^{2} \Pi_{3 / 2, u}$ and peak no. 2 for $\mathrm{A}^{2} \Pi_{1 / 2, u}$ in TKER. Comparing with Cockett et al. [4], who identified vibrational levels for the $\mathrm{j}=3 / 2$ case, the vibrational level is $\mathrm{v}^{\prime}=17$. Because of the uncertainty in the scaling of one vibrational quantum of the $\mathrm{X}^{2} \Pi_{1 / 2, g}$ state, here a uncertainty of two vibrational quanta remains, since the vibrational constant $\omega$ is for the $\mathrm{A}^{2} \Pi_{u}$ states $\left(\omega\left(\mathrm{A}^{2} \Pi_{3 / 2, u}\right)=0.0171 \mathrm{eV}[4], \omega\left(\mathrm{A}^{2} \Pi_{1 / 2, u}\right)=0.0148 \mathrm{eV}[9]\right)$ approximately half of the value for the $\mathrm{X}^{2} \Pi_{g}$ states $\left(\omega\left(\mathrm{X}^{2} \Pi_{3 / 2, g}\right)=0.0298 \mathrm{eV}\right.$ and $\left.\omega\left(\mathrm{X}^{2} \Pi_{1 / 2, g}\right)=0.0284 \mathrm{eV}[4]\right)$. Although only an approximate assignment can be made for the $\mathrm{A}^{2} \Pi_{1 / 2, u}$ state, it obvious that the $\mathrm{A}^{2} \Pi_{1 / 2, u}$ state is peaked at a rather low vibrational state. This is due to the simple fact that the excitation energy here does only allow two vibrational quanta more $(\omega=0.0148 \mathrm{eV}[9])$.

\subsubsection{Unknown state}

An ionization process is apparent in the image with a TKER of $0.48 \mathrm{eV}$. By comparison with measurements from different days it shows that it has a fixed intensity ratio with the $\mathrm{A}^{2} \Pi_{u}$ states, but not with the $\mathrm{X}^{2} \Pi_{g}$ states, thus it must be initiated with the same photon combination as the $\mathrm{A}^{2} \Pi_{u}$ states, which is two UV photons at $212.55 \mathrm{~nm}$ wavelength. This gives a transition 
energy of $11.14 \mathrm{eV}$. So far, this state never showed up in the conventional photoelectron experiments in literature. The next well know state is the predissociating $\mathrm{B}^{2} \Sigma_{g}^{+}$state which is with a transition energy of $12.95 \mathrm{eV}$ out of reach.

Cockett et al. [4] identified a hitherto unknown state, $a^{4} \Sigma_{3 / 2, u}^{-}(2421)$ with a transition energy of $10.66 \mathrm{eV}, 0.54 \mathrm{eV}$ below the state measured in this work. Probably because the absorption of two photons is normally necessary to achieve the configuration change which includes two orbitals, this state was not observed yet. Most older experiments used either electron collisions or high energy He(II) lines or synchrotron radiation where only one photon was responsible for the ionization process.

They base their assignment of the new state on calculation of $\mathrm{Li}$ and Balasubramanian [10], which were at that time the only available calculations of the $\mathrm{I}_{2}^{+}$potentials which were sufficiently precise. Li and Balasubramanian predicted a state with ${ }^{4} \Sigma_{u}^{-}$symmetry in this region. Because of the closer proximity of the $\mathrm{j}=3 / 2$ spin orbit component of this quartet state to the $\mathrm{A}^{2} \Pi_{3 / 2, u}$ state, than of the $\mathrm{j}=1 / 2$ component of the quartet state to the $\mathrm{A}^{2} \Pi_{1 / 2, u}$, they believed that intensity borrowing would be more likely for the $\mathrm{j}=3 / 2$ component. This interaction of states is needed to allow for the transition from the doublet to the quartet state. Thus they assigned the found state to the $a^{4} \Sigma_{3 / 2, u}^{-}$. Nevertheless, since they found unassigned structure in their spectra, they do not exclude the possibility that the $\mathrm{a}^{4} \Sigma_{1 / 2, u}^{-}$state is excited too. The latter state was predicted by Li and Balasubramanian to lie at $1.02 \mathrm{eV}$ above the ground state, resulting in $10.33 \mathrm{eV}$.

Another possibility of the state found in this work would be higher lying states ${ }^{2} \Delta_{u}(2421)$ and ${ }^{2} \Sigma_{u}^{+}(2421)$, which are predicted to be at $1.51 \mathrm{eV}$ (total $10.82 \mathrm{eV}$ ) and $1.79 \mathrm{eV}$ (total $11.10 \mathrm{eV}$ ) respectively above the ground state. But these states have a considerably longer bondlength $\left(\mathrm{r}_{e} \approx 3.5 \AA\right)$ than the neutral ground state with $2.66 \AA$ and are unlikely to be reached from there with a direct ionization process, at least they should exhibit considerable vibrational excitation, thus the lower lying state is more likely.

However, new relativistic calculations were performed by de Jong et al [11]. And in the light of their calculations they came to a somewhat different conclusion concerning the assignment of the newly found state. From the relative position of the potential curves and agreement of the calculated spectroscopic data with measured properties, they assigned the newly found state to $\mathrm{a}^{4} \Sigma_{1 / 2, u}^{-}$, which is in their calculation only $1.64 \mathrm{eV}(10.947 \mathrm{eV})$ above the ground state, whereas the ${ }^{4} \Sigma_{3 / 2, u}^{-}$state lies at $2.09 \mathrm{eV}(11.397 \mathrm{eV})$. As well the above mentioned ${ }^{2} \Delta_{u}$ state changes to $2.57 \mathrm{eV}(11.877 \mathrm{eV})$ above ground state. 
Another possibility is that actually the state with $11.14 \mathrm{eV}$ transition energy is the $\mathrm{A}^{2} \Pi_{3 / 2, u}$ state, and that the one at $10.98 \mathrm{eV}$ belongs to a different state, although it is more likely that the state with a higher intensity (compare fig. 13.3) is the $\mathrm{A}^{2} \Pi_{3 / 2, u}$ state.

Since the measured state in this work is somewhere in between these numbers, no clear assignment can be made.

The orbital configuration of the valence electrons of the molecular iodine states in table 13.2 are given in the notation by Mulliken [7] in klmn meaning $\sigma_{g}^{k} \pi_{u}^{l} \pi_{g}^{* m} \sigma_{u}^{* n}$ and in potential energy relative to the ground state. Obviously, the potential values have changed considerably in the newer calculations, sometimes by $1 \mathrm{eV}$ relative to the first ionization limit. Because of the high amount of coupling in the heavy iodine molecule, which cannot yet be fully incorporated in calculations, it is to be expected that these results will change further in the future.

\subsubsection{Angular distribution of the photoelectrons}

One thing about the photoelectron images strikes as odd, which is the lack of any peaked angular distribution. Exact figures for the $\beta$-parameter can be taken from table 13.1. All of them are close to zero.

These results are in a way rather surprising. Although an isotropic distribution is not impossible, it is doubtful that this represents the real distribution. Eland discusses some main principles of angular distribution in photoelectron spectroscopy in his book [12], which can be applied to the problem here. Firstly, the angular distribution depends mostly on the orbital which loses the electron, therefore states resulting from the same electronic configuration should have similar $\beta$-parameters. Since all $\beta$-parameters are close to zero, this is at least the case. But, secondly, the nature of the electronic orbital must be taken into account. A ionization from an atomic s orbital gives a $\beta$-parameter of 2 . For molecules, similar rules hold. A ionization from an $\sigma$-orbital should give relatively high $\beta$-values. As an example, the $1 \mathrm{~s} \sigma_{g}$ ionization of $\mathrm{H}_{2}$ give $\beta=1.8$. The higher the 1 number of the corresponding orbital, the lower the $\beta$-value should be. This is because the outgoing wave of the electron can be understood as something similar to the wave functions of the electron in the hydrogen atom. Going from a $\mathrm{s}$ to a $\mathrm{p}$ state gives the wavefunction a $\mathrm{p}$ character which is proportional to a $\cos ^{2}$. A transition from a $\mathrm{p}$ state would result in a superposition of a $\mathrm{s}$ and a $\mathrm{d}$ wave, with the s wave being isotropic and the $\mathrm{d}$ wave peaked along the electric vector of the laser light, but not as much as the $\mathrm{p}$ function. This can in principle be continued to higher 1 states. Since both transition from the ground state of the molecule to the $\mathrm{X}^{2} \Pi_{g}$ and $\mathrm{A}^{2} \Pi_{u}$ state of the cation includes the ionization 


\begin{tabular}{|l||l|c|l|c|}
\hline $\begin{array}{l}\text { assigned } \\
\text { state }\end{array}$ & $\begin{array}{l}k l m n \\
{[11]}\end{array}$ & $\begin{array}{c}\text { energy } \\
{[11]}\end{array}$ & $\begin{array}{l}k l m n \\
{[10]}\end{array}$ & $\begin{array}{c}\text { energy } \\
{[10]}\end{array}$ \\
\hline \hline $\mathrm{X}^{2} \Pi_{3 / 2, g}$ & $2430^{2} \Pi_{g}$ & 0 & $2430^{2} \Pi_{g}$ & 0 \\
& & & $0432^{2} \Pi_{g}$ & \\
\hline $\mathrm{X}^{2} \Pi_{1 / 2, g}$ & $2430{ }^{2} \Pi_{g}$ & 0.65 & $2430^{2} \Pi_{g}$ & 0.74 \\
& & & $0432^{2} \Pi_{g}$ & \\
\hline $\mathrm{A}^{2} \Pi_{3 / 2, u}$ & $2340^{2} \Pi_{u}$ & 1.71 & $2340^{2} \Pi_{u}$ & 1.26 \\
& $2421^{4} \Sigma_{u}^{-}$ & & $1431^{2} \Pi_{u}$ & \\
\hline $\mathrm{A}^{2} \Pi_{1 / 2, u}$ & $2340^{2} \Pi_{u}$ & 2.55 & $2340^{2} \Pi_{u}$ & 1.86 \\
& $2421^{4} \Sigma_{u}^{-}$ & & $1431^{2} \Pi_{u}$ & \\
& & & $2421^{4} \Sigma_{u}^{-}$ & \\
\hline $\mathrm{a}^{4} \Sigma_{1 / 2, u}^{-}$ & $2421^{4} \Sigma_{u}^{-}$ & 1.64 & $2421^{4} \Sigma_{u}^{-}$ & 0.99 \\
& & & $2241^{4} \Sigma_{u}^{-}$ & \\
& & & $2421^{2} \Sigma_{u}^{+}$ & \\
\hline $\mathrm{a}^{4} \Sigma_{3 / 2, u}^{-}$ & 2421 & 2.09 & $2421^{4} \Sigma_{u}^{-}$ & 1.02 \\
& $2340{ }^{2} \Pi_{u}$ & & $2241^{4} \Sigma_{u}^{-}$ & \\
\hline $\mathrm{B}^{2} \Sigma_{g}^{+}$ & $1440{ }^{2} \Sigma_{g}^{+}$ & 3.97 & $1440^{2} \Sigma_{g}^{+}$ & 2.4 \\
& 2331 & & $2331^{2} \Sigma_{g}^{+}$ & \\
\hline${ }^{2} \Sigma_{u}^{+}$ & & & $2421^{2} \Sigma_{u}^{+}$ & 1.79 \\
& & & $2241^{2} \Sigma_{u}^{+}$ & \\
\hline${ }^{2} \Delta_{u}$ & $2421^{2} \Delta_{u}$ & 2.57 & $2421^{2} \Delta_{u}$ & 1.51 \\
& & & $2241^{2} \Delta_{u}$ & \\
\hline
\end{tabular}

Table 13.2: Calculated states of $I_{2}^{+}$and their contributing electron configurations. The population of the molecular orbits are given in the klmn notation by Mulliken [7], meaning $\sigma_{g}^{k} \pi_{u}^{l} \pi_{g}^{* m} \sigma_{u}^{* n}$. Calculated term values in [eV] above the first ionization limit.

of a bonding and antibonding $\mathrm{p}$ orbital respectively, the case with an outgoing $\mathrm{s}$ and $\mathrm{d}$ wave would apply here. If the $\beta$ parameters really represents this effect, mainly s waves would be included in the transitions. Thirdly, in the range of UV photoelectron spectroscopy, the $\beta$-parameter changes rather quickly and only reaches constancy with kinetic energy release of $50 \mathrm{eV}$ and more. Eland states that especially for KER of less than $5 \mathrm{eV}$ this effect is most pronounced, therefore it is rather surprising that electrons with a KER in the range from nearly $0 \mathrm{eV}$ to around $1 \mathrm{eV}$ and resulting from two different orbits should have very similar angular distributions. Actually, it is less the fact, that they are very similar that strikes as odd. If they had all shown a asymmetric behaviour, it would be merely interesting and had inspired more 
research, but that they should all exhibit an isotropic behaviour seems unlikely. This points more in the direction that by some mechanism, the initial angular distribution was destroyed.

But if the angular distribution is destroyed by some external effect, possibly a magnetic or electric field in the chamber, a destruction of the entire image would be expected.

If by the incident light a long lived autoionizing state is excited, the angular distribution will be lost and after some rotational periods, the measured $\beta$-parameter will be effectively zero. It is already known that electronic autoionization of $\mathrm{A}^{2} \Pi_{u}$ state based Rydberg states contributes significantly to the ionization of $\mathrm{I}_{2}$ [13]. This is in line with the excitation to several vibrational quanta of the $\mathrm{X}^{2} \Pi_{g}$ state and the fact that the ionization energy is not at the threshold but considerably higher. The $\mathrm{A}^{2} \Pi_{1 / 2, u}$ state, which has the smallest TKER and is therefore nearest to its ionization threshold, should be most susceptible to direct ionization. Indeed, it has only a FWHM of $0.016 \mathrm{eV}$, which is approximately its $\omega$ value $(0.0148 \mathrm{eV})$ and exhibits the highest $\beta$-value with 0.22 .

Nevertheless, although there are higher lying Rydberg states belonging to excited ionic states which can couple onto the lower lying ionic states, it is even with the existing high density of these Rydberg states surprising that for all five observed ionization processes corresponding Rydberg states are met by chance.

\section{Bibliography}

[1] B.R. Higginson, D.R. Lloyd, and P.J. Roberts. Chem. Phys. Lett., 19:480, 1973.

[2] J.C. Miller. Jour. Phys. Chem., 91:2589, 1987.

[3] K.P. Lawley, A.C. Flexen, R.J. Maier, A. Manck, T. Ridley, and R.J. Donovan. Phys. Cem. - Chem. Physics, 4:1412, 2002.

[4] M.C.R Cockett, R.J. Donovan, and K.P. Lawley. Jour. Chem. Phys., 105:3347, 1996.

[5] K.P. Huber and G. Herzberg. Molecular spectra and molecular structure; IV. Constants of diatomic molecules. Van Nostrand Reinhold, 1979.

[6] G. Herzberg. Molecular spectra and molecular structure; I. Spectra of diatomic molecules. Van Nostrand Reinhold, 1950.

[7] R.S. Mulliken. Jour. Chem. Phys., 55:288, 1971. 
[8] M.C.R Cockett, J.G. Goode, K.P. Lawley, and R.J. Donovan. Jour. Chem. Phys., 102:5226, 1995.

[9] A.J. Yencha, M.C.R Cockett, J.G. Goode, R.J. Donovan, A. Hopkirk, and K.P. Lawley. Chem. Phys. Lett., 229:347, 1994.

[10] Junqing Li and K. Balasubramanian. Jour. Molec. Spec., 138:162, 1989.

[11] W.A. de Jong, L. Visscher, and W.C. Nieuwpoort. Jour. Chem. Phys., 107:9046, 1997.

[12] J. H. D. Eland. Photoelectron spectroscopy. Butterworths, 1984.

[13] Y.-Y. Gu, A.M. Chojnacki, C.J. Zietkiewicz, A.A. Senin, and J.G. Eden. Jour. Chem. Phys., 119:12342, 2003. 


\section{Chapter 14}

\section{Results at VUV wavelength}

\subsection{Experimental results}

The images of iodine atomic cations in this section were recorded with Lyman- $\alpha(D)(\lambda=121.53 \mathrm{~nm}[1]$ or $10.20 \mathrm{eV})$ and residue $212.55 \mathrm{~nm}(5.83 \mathrm{eV})$ wavelength laser light present.

Typical raw and converted images from different days are shown in the images below, the raw images are on the left hand side, the converted images are on the right hand side. It is obvious that under varied conditions, different features of the images become more pronounced. The main issue which will be addressed in the following sections is by which mechanism the different features are created.

The experimental results are summarized in table 14.1 below.

The TKER distributions are shown in figure 14.2. Here all nine TKER distributions are included which were used to determine the experimental results. In contrast to the UV+VUV TKER distribution shown in chapter 15 , the distributions are here normalized so that the peak at $0.41 \mathrm{eV}$ TKER has a height of unity. Because a number of processes are involved which depend on different photon combinations, a normalization to the same area is meaningless. With the normalization to one peak a qualitative comparison by visual inspection is easier. It is obvious to the naked eye that the intensity of some peaks changes dramatically relative to the intensity of the $0.41 \mathrm{eV}$ peak. 


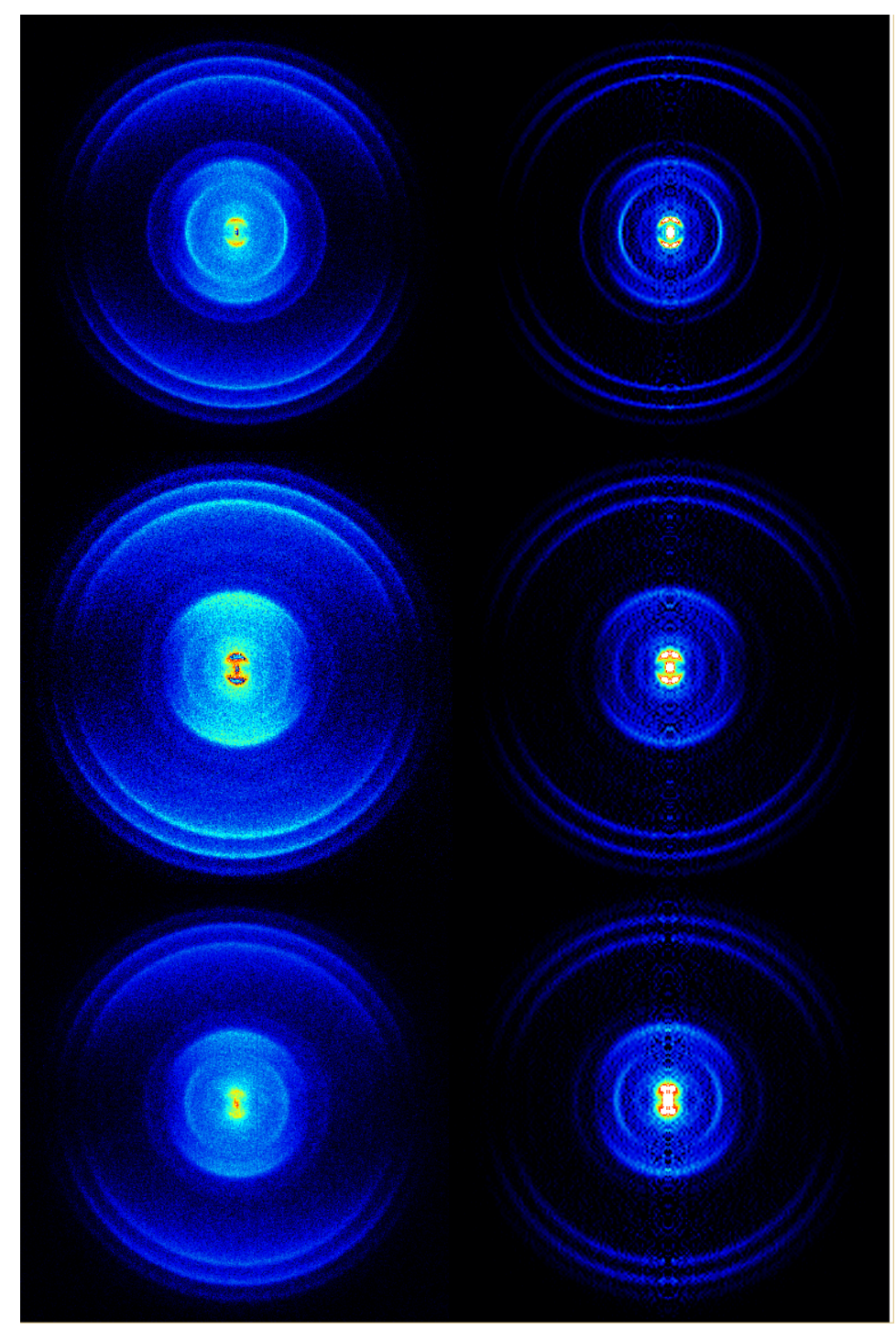

Figure 14.1: Raw and converted images at $121.53 \mathrm{~nm}$ wavelength with $212.55 \mathrm{~nm}$ residue wavelength. Raw images are on the left, converted images are on the right hand side. A selection of images is shown which illustrate the varying features under different conditions of the laser power. As an example, note the isotropic ring and the ring showing a mainly perpendicular angular distribution, both can be found in the cluster of rings in the centre of the image. In the uppermost image, these rings are quite pronounced, in the middle image they nearly disappear and the lowest picture, their intensity is intermediate. The conditions leading to different behaviour are discussed in detail in section 14.2.3 and 14.2.4. 


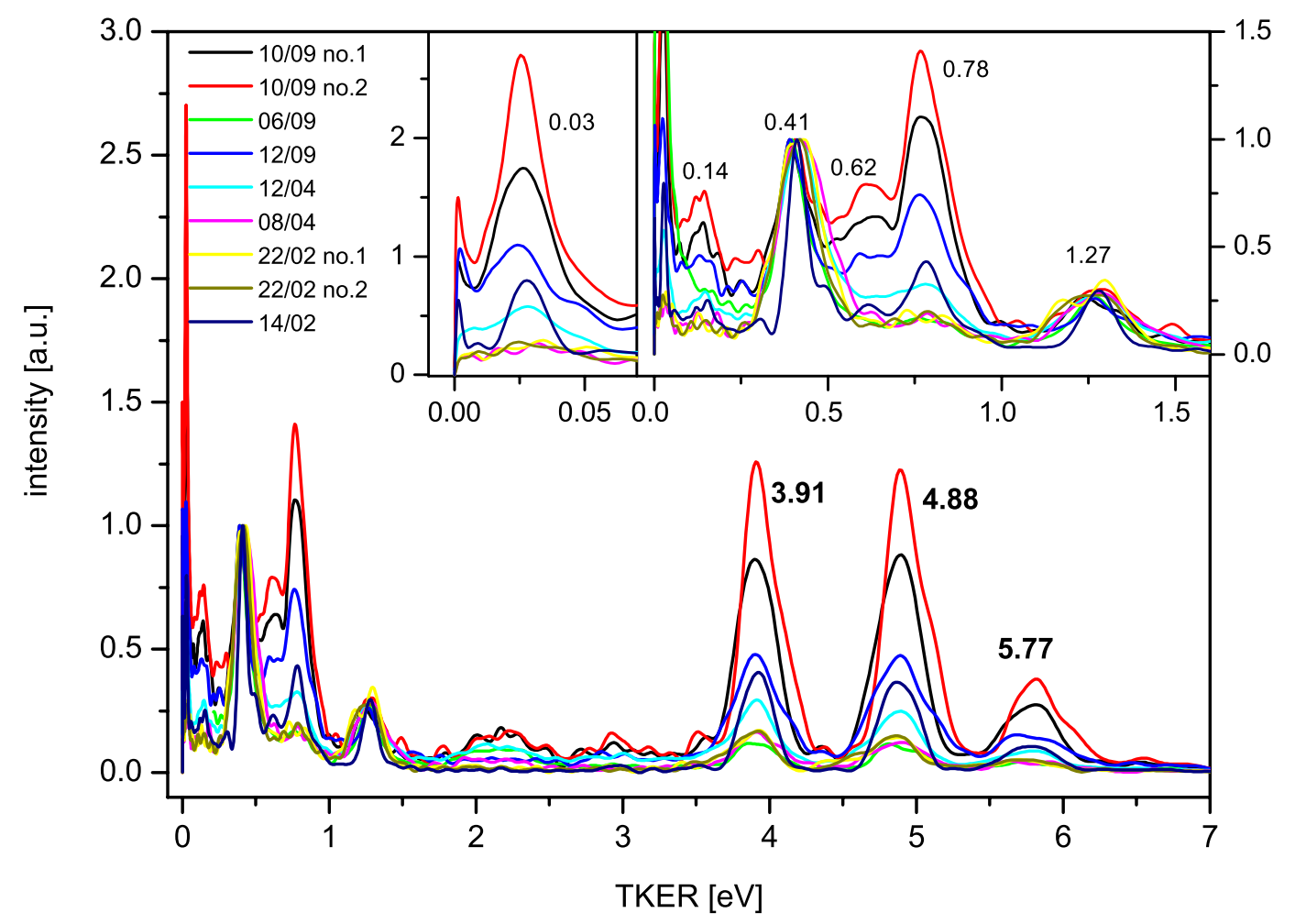

Figure 14.2: TKER distributions at $121.53 \mathrm{~nm}$ wavelength with $212.55 \mathrm{~nm}$ residue wavelength. All distributions are shown which were used to determine the experimental results. The TKER distribution labelled 06/09 is only shown from $0.3 \mathrm{eV}$ onwards, because below that value it is dominated by a molecular ion signal which was insufficiently suppressed in this measurement. Distributions are normalized so that the peak at $0.41 \mathrm{eV}$ equals one.

\subsection{Additional experiments}

One channel can be established unambiguously with a single additional experiment. Instead of the $\mathrm{I}^{+}$ions, the ion lens and the detector was set to detect $\mathrm{I}^{-}$ions. Two rings exhibited analogous $\mathrm{I}^{-}$channels, these are the rings at 0.41 and $1.27 \mathrm{eV}$ TKER. Hence they can be clearly assigned to dissociation of molecular iodine to free ion pair states. This issue is discussed in a separate chapter 16 .

The other rings were more difficult to place. Most of the rings exhibited a similar $\beta$-parameter indicating a mostly parallel transition as well as a similar width. The $\beta$-parameters are shown in figure 14.3. A closer inspection in fig. 14.3 reveals that mostly all $\beta$-parameters from one measurement are high or low compared to the others, i.e if it is comparatively low for one peak 


\begin{tabular}{|c|c||c|c|c|c|c|c|}
\hline $\begin{array}{c}\text { peak } \\
\text { no. }\end{array}$ & $\begin{array}{c}\text { experimental } \\
\text { value }[\mathrm{eV}]\end{array}$ & $\begin{array}{c}\text { width } \\
{[\mathrm{eV}]}\end{array}$ & $\begin{array}{c}\text { width } \\
\text { pixel }\end{array}$ & $\begin{array}{c}\text { range } \\
{[\mathrm{eV}]}\end{array}$ & $\beta$ & $\begin{array}{c}\text { range } \\
\beta\end{array}$ & photons \\
\hline 1 & 0.03 & 0.04 & 6.8 & \pm 0.005 & 1.17 & 0.58 & $2 \mathrm{VUV}$ \\
2 & 0.14 & 0.12 & 9.6 & \pm 0.01 & 0.26 & 0.49 & $\mathrm{VUV}+\mathrm{UV} ?$ \\
3 & 0.41 & 0.14 & 7.1 & \pm 0.02 & -0.38 & 0.49 & $1 \mathrm{VUV}$ \\
4 & 0.62 & 0.13 & 5.7 & \pm 0.02 & 0.87 & 0.68 & $2 \mathrm{VUV}$ \\
5 & 0.78 & 0.21 & 7.8 & \pm 0.02 & 1.34 & 0.95 & $2 \mathrm{VUV}$ \\
6 & 1.27 & 0.22 & 6.7 & \pm 0.02 & 0.09 & 0.57 & $1 \mathrm{VUV}$ \\
7 & 2.16 & 0.61 & 13.8 & \pm 0.07 & 1.20 & 0.52 & VUV $+\mathrm{UV}$ \\
8 & 3.91 & 0.34 & 5.9 & \pm 0.02 & 1.93 & 0.69 & $2 \mathrm{VUV}$ \\
9 & 4.88 & 0.40 & 6.3 & \pm 0.05 & 1.92 & 0.75 & $2 \mathrm{VUV}$ \\
10 & 5.77 & 0.55 & 7.9 & \pm 0.09 & 1.59 & 0.86 & $2 \mathrm{VUV}$ \\
\hline
\end{tabular}

Table 14.1: The experimental results in this work, their corresponding $\beta$ parameters and the production mechanism they are assigned to. Range [eV] means the maximum value by which the peak position deviated from the average value all used images considered. Range $\beta$ describes the difference between the maximum and minimum value determined in different measurements for one peak.

compared to measurements on different days, it is usually also low for all other peaks. The average value is indicated by a square with the peak number, drop lines are included to guide the eye. The range of $\beta$-parameter given in table 14.1 describes the difference between the maximum and minimum value determined in different measurements for one peak. Peak no. 3 and no. 6 are the free ion pair states at at 0.41 and $1.27 \mathrm{eV}$ TKER which exhibit nearly isotropic $\beta$-parameters. Apart from them, only the ring no. 2 at $0.14 \mathrm{eV}$ gives a similarly isotropic angular distribution.

Because the average width was determined in kinetic energy which depends on the square of the speed distribution which is actually measured in the experiment, the value rises in a first approximation quadratically. The real widths of the speed distribution which has the real unit 'pixel' varies with different settings of the electrostatic lens, see chapter 11 . Thus the average width in pixel was not calculated from all different widths taken from the original speed distribution but was retransformed from the average width in kinetic energy to a width in pixel with a normed transformation factor. This provides a set of widths which are directly comparable to each other, see table 14.1. Actually, these values represent the real values quite well, which were spot checked in some of the images. The most remarkable deviation is the width of the peak at $2.16 \mathrm{eV}$. The one at $0.14 \mathrm{eV}$ is as well to be slightly out of line. Nevertheless, with smaller translational energy, the image gets 
rather crowded and it is difficult to assign an unambiguous width and area to an individual peak.

Similar values in those quantities width and $\beta$-parameter can be an indication of a similar origin.

Further experiments were made to establish dependencies of the results on the two available wavelength and on the number of the absorbed photons.

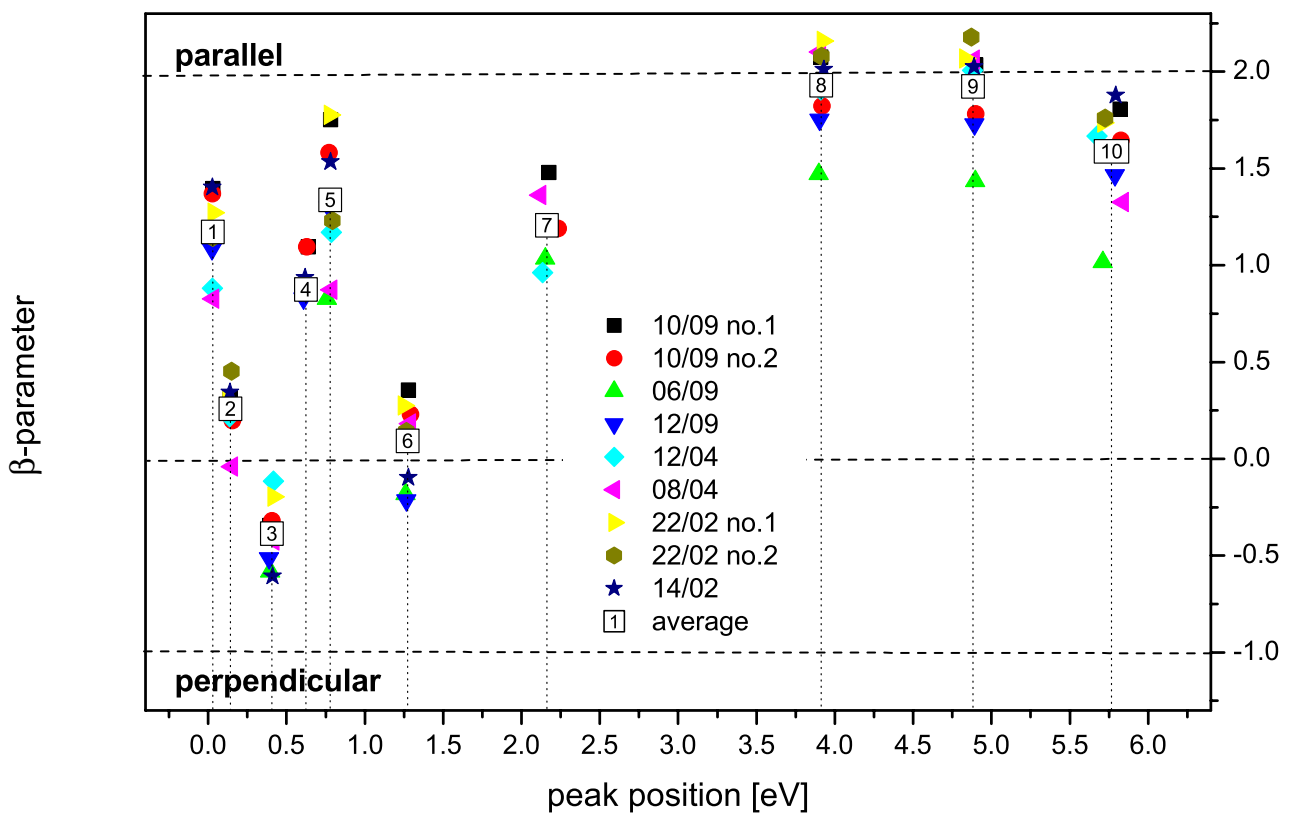

Figure 14.3: $\beta$-parameter at $121.53 \mathrm{~nm}$. Vertical drop lines from the average value are shown to guide the eye.

\subsubsection{UV residue}

Because the VUV production has only a maximum efficiency of 5\% [2], 0.5$1 \mathrm{~mJ}$ per pulse UV light is still present in the VUV beam. The focal length of the lens which admits the VUV and also the UV beam from the VUV cell into the chamber does not differ that much for the UV and VUV, which means that the UV laser light is sufficiently focussed in the dissociation region.

The spatial overlap of the two laser beams is only given in the VUV cell, but then depends rather arbitrarily on where exactly and under which angle both laser beams hit the lens which admits the VUV and UV light into the vacuum chamber. Thus the exact spatial overlap in the dissociation region, i.e. within the electrostatic lens, is a matter of coincidence and it must be 
distinguished between processes depending solely on UV photons and those depending on a combination of a UV and a VUV photon.

The ring at $2.163 \mathrm{eV}$ TKER does only show up on some days which may hint at an accidental overlap of the two beams and is thus a good candidate for a process of a combined VUV and UV photon. Unfortunately, the overlap could not be checked with the movable mask, because the mask could only be used in the electrostatic lens for an approximate adjustment. To receive a reasonably high enough signal and, even more important, a good quality of the image, the laser beam was then adjusted to an optimal position which was slightly removed from the pinhole, using the single shot images taken by the CCD-camera as a reference.

To differentiate further between dissociation processes initiated by VUV and UV, one has to take into account that the VUV power does not solely depend on the UV power but as well on the IR power and exact wavelength, spatial and focal overlap of the two beams, correct meeting of the krypton resonance condition, etc. Therefore no simple fixed power relation between the UV and VUV exists, and for different conditions on different days, the intensity ratio between processes depending only on VUV light and only on UV light or a combination of both should vary.

\subsubsection{IR residue in the VUV beam}

When producing VUV at Lyman- $\alpha$ wavelength by four frequency mixing, UV light at $212.55 \mathrm{~nm}$ wavelength is needed to satisfy the resonant transition in krypton. The resulting VUV wavelength is determined by the subtracted photon, which is at $\approx 844 \mathrm{~nm}$ wavelength, see 3 . Thus also the residue IR radiation should be given some thought.

Firstly, the IR beam is usually rather weak. Only the oscillator is used in the according dye laser, giving around $200 \mu \mathrm{J}$ per pulse, which is sufficient for the VUV production. Additionally, the lens which introduces the VUV beam into the chamber has a focal length which is optimally adapted to focus the VUV laser light onto the dissociation point to produce a high photon density and a small source volume. Since the IR has a wavelength far from the VUV, the focal length is notedly different and the highest density of IR photons does not coincide with the source volume within the electrostatic lens.

For the dissociation process this means that the IR beam can be discounted as a possible origin for any process. The energy of one IR photon with $1.47 \mathrm{eV}$ is too low to achieve e.g. dissociation of the neutral molecule $\left(\mathrm{E}_{\text {diss }}=1.54 \mathrm{eV}\right)$ or the cationic molecule $\left(2.688 \mathrm{eV}\right.$ for $\mathrm{X}^{2} \Pi_{3 / 2, g}, 2.044 \mathrm{eV}$ for $\left.\mathrm{X}^{2} \Pi_{1 / 2, g}\right)$, which can be generated with one VUV photon, and because of the low intensity multiphoton absorption is extremely improbable. 


\subsubsection{Polarization}

A way to check which wavelengths are involved in the dissociative step is the polarization. It is inherent in velocity map imaging that the polarization of the involved laser beams has to be in the plane of detection or the angular information is lost. If the polarization is perpendicular to the plane of detection, the effect is quite noticeable. Either the ring seem to disappear entirely, especially for rings with low intensity and a preferable parallel transition moment, or, for high intensity and a preferable perpendicular transition moment, the ring is still visible, but the angular distribution information is lost and the ring appears to belong to an isotropic distribution. Now it is possible to change the polarization of the VUV independently of the UV by turning the polarization of the IR laser. This was done and the change observed.

The rings at the two lowest TKER $($ TKER $\leq 0.14 \mathrm{eV}$ ) were overshadowed by the molecular ion signal, thus they are excluded from the following reasoning. The $0.62 \mathrm{eV}$ ring was not visible during these measurements.

From the visible rings, only three remained after changing the polarization, two of them the ones belonging to ion pair dissociation, which lose their angular information. Only one stayed completely unchanged, which is the one at $2.16 \mathrm{eV}$ TKER. This means that the symmetry of the transition is dominated by a $212.55 \mathrm{~nm}$ photon. Together with the consideration from the above subsection, it is attributed to a process initiated by a combination of one UV and one VUV photon, giving a total excitation energy of $16.03 \mathrm{eV}$. Because of the similarity to the processes with VUV+365 nm and VUV+266 nm wavelength, it is discussed in the appropriate chapter 15 together with the latter processes. The dissociative step of all other processes seem to be caused by a VUV photon.

\subsubsection{Change in VUV intensity}

With these dependencies on the polarization of the VUV established, the change of peak intensity with the change in laser intensity is checked. Here, the VUV light is tuned to a difference wavelength (Lyman- $\alpha(H))$. Since the VUV production is optimised for Lyman- $\alpha(\mathrm{D})$ by the argon-krypton ratio, the VUV intensity can be changed by tuning the IR wavelength and with it the VUV wavelength. The VUV intensity change goes without changing the profiles nor the intensities of the residue laser. Both images were taken with the same amount $(16,000)$ of laser shots per image. A comparison of the 
TKER distribution of both cases is shown in figure 14.4. It is observed that the intensity of some peaks change directly proportional to the VUV power, these are the ones which belong to the free ion pair channels at 0.41 and $1.27 \mathrm{eV}$ TKER. The peaks at 3.91, 4.88 and $5.77 \mathrm{eV}$ TKER clearly depend with a squared proportion on the VUV laser power. This indicates that the former are initiated by one VUV photon and the latter by two respectively. The factor by which the integrated signal of the peaks Lyman- $\alpha(\mathrm{H})$ dropped compared to the signal at Lyman- $\alpha(\mathrm{D})$ is given in table 14.2 below. As well we see that the dependency on the VUV transitions is obviously not resonant.

Unfortunately, for the very weak peaks with low TKER it is not possible to establish a unimpeachable correspondency. The peaks at 0.03 and $0.78 \mathrm{eV}$ TKER are likely to belong to a two VUV photon absorption, whereas the

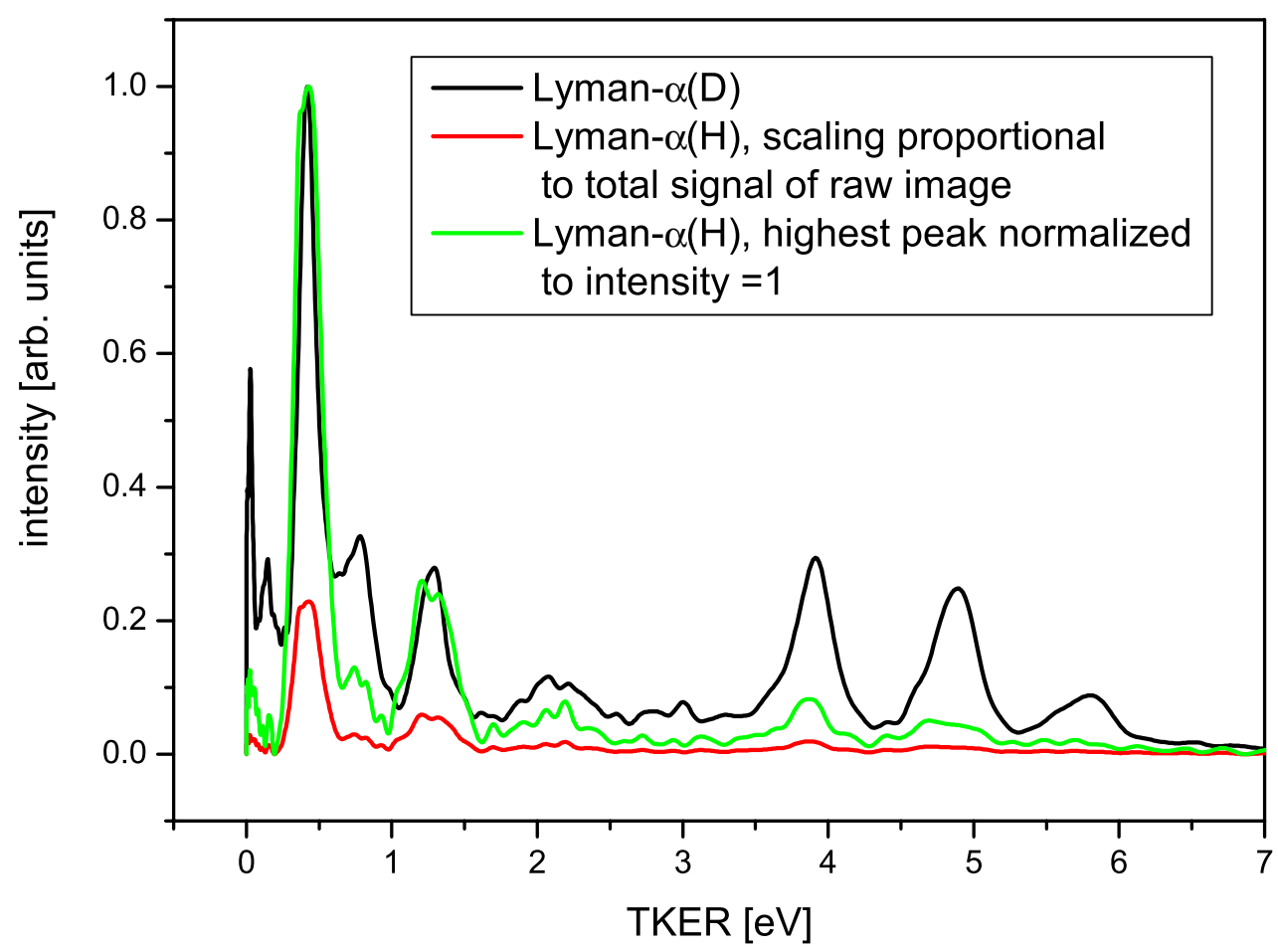

Figure 14.4: Comparison of the TKER distribution at Lyman- $\alpha(D)$ $121.53 \mathrm{~nm}$ and Lyman- $\alpha(H) 121.57 \mathrm{~nm}$. Both images were taken with 16,000 shots. The distribution for Lyman- $\alpha(H)$ is shown twice. Once the total signal is scaled in comparison to Lyman- $\alpha(D)$ according to the ratio of the total signal of the respective raw images. For the second representation of the distribution at Lyman- $\alpha(H)$, the intensity of highest peak at $0.41 \mathrm{eV}$ was set as one, identically to the Lyman- $\alpha(D)$ distribution shown here and above 14.2. 


\begin{tabular}{|c||c|c|c|c|c|c|c|c|c|}
\hline peak & 0.03 & 0.14 & 0.41 & 0.78 & 1.27 & 2.16 & 3.91 & 4.88 & 5.77 \\
\hline factor & 17.9 & 2.1 & 3.6 & 11.5 & 4.3 & 8.5 & 15.6 & 17.4 & 15.3 \\
\hline
\end{tabular}

Table 14.2: Scaling factors of the integrated signal when VUV wavelength is tuned from Lyman- $\alpha(D)$ to Lyman- $\alpha(H)$.

one at $0.14 \mathrm{eV}$ seems more to be caused by one VUV photon. That the factor is actually even much lower than $\approx 4$ is probably caused by the overall low signal of the peak and the insufficient resolution from the next peak.

If in doubt, various possibilities will be shortly discussed when appropriate.

A third possibility to check which peak depends on which photon combination, is to compare the ratios of the peak. Where peaks exhibit fixed ratios between their respective integrated signal or area, they depend on the same photon combination, because then these ratios are independent of the daily varying conditions. In figure 14.5 the integrated signal of the peaks are given. Always the area of the peak given in bold letters in the panels is normalized to one. If the area of other peaks stands in a fixed relation to it, the entries from different days will be very close to each other for these peaks. The peak normalized to one and the peaks which definitely have a fixed ratio to it are indicated with a dashed vertical line in one panel, peaks which may have a fixed ratio to these are shown with dotted vertical line. These comparisons mainly corroborate the conclusions from above, but additionally a possible relationship between the $0.14 \mathrm{eV}$ and the $2.16 \mathrm{eV}$ peak can be made out. It is likely the $0.14 \mathrm{eV}$ peak depends also on a VUV+212.55 nm combination. The peak at $0.62 \mathrm{eV}$ was now attributed to two VUV photons.

Now with these correspondencies established, we can proceed to an exact analysis of the mechanism.

\subsection{Results and discussion}

Apart from the free ion pair formation, which is actually a rather exotic way to produce ions and was only observed in some diatoms, more obvious ways of obtaining iodine ions in a photochemistry experiment come to mind. One possibility is the dissociation of the neutral molecule into neutral fragments. Any atomic iodine level except of the ground state can be subsequently ionized by another one of the available photons. A second possibility is that the neutral molecule is first ionized to a stable $\mathrm{I}_{2}^{+}$state (first ionization potential $9.307 \mathrm{eV}$ ) with one VUV photon or two UV photons, and then dissociated by another photon. A third option which will be considered is that the neutral 


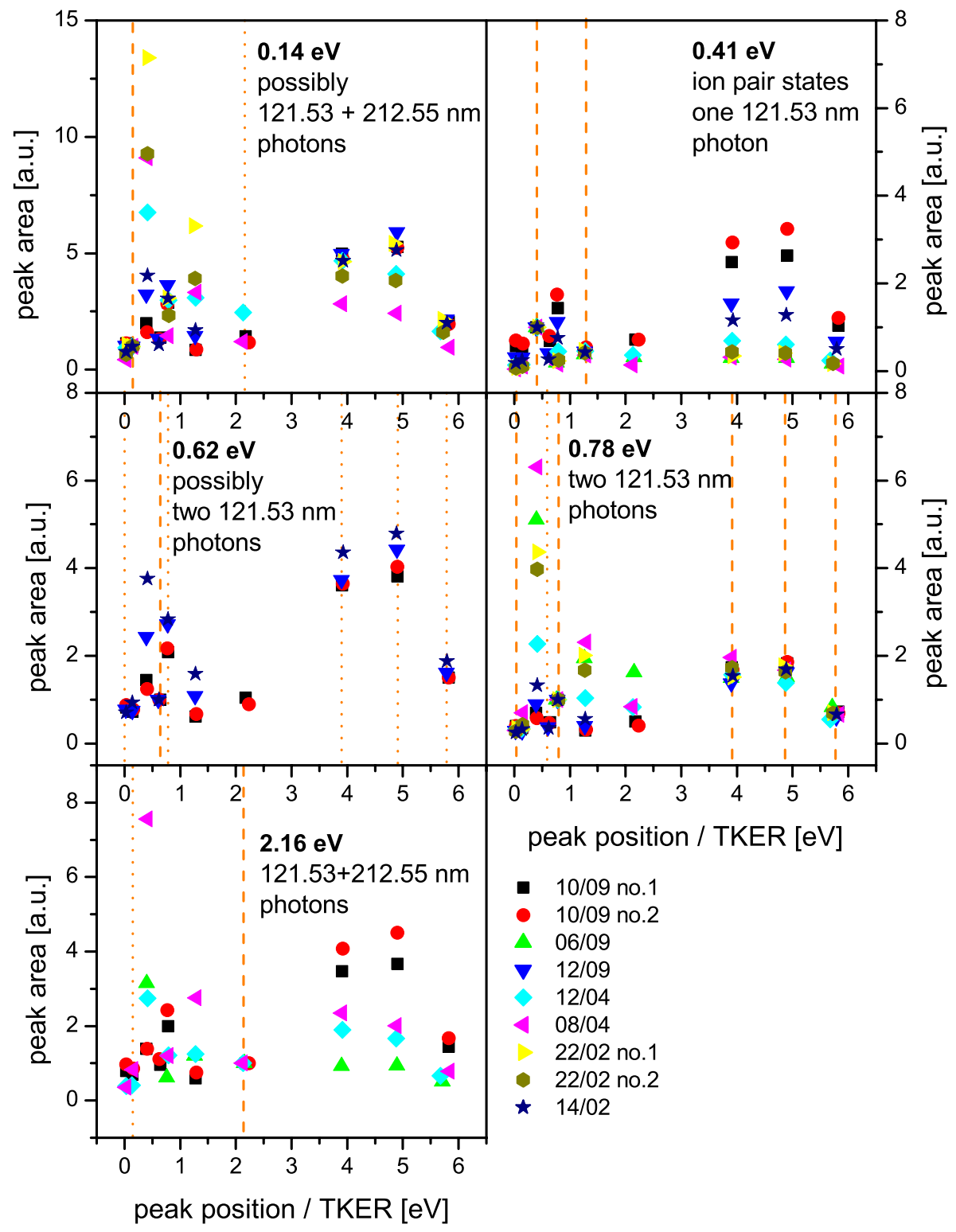

Figure 14.5: Comparison of the integrated signal of the peaks. In each panel the area of the peak given is normalized to one. Then this one and all others displaying a fixed intensity ratio to it are indicated with a vertical dashed line. The dotted line indicates peaks where the area seems to be in a fixed ratio to those, but cannot be said for sure because of low signal or few data points.

molecule undergoes transition to an dissociative ionic state by the absorption of two photons. 
These considerations together with the results of those further experiments described above will be used to clarify the fragmentation mechanism.

\subsubsection{Peaks at low TKER}

These are the rings at $0.03,0.14,0.62$ and $0.78 \mathrm{eV}$. The division of the observed rings into processes with a low and high TKER is of course an arbitrary choice, since there is no absolute definition of high or low TKER. For example, the highest observed TKER of $5.77 \mathrm{eV}$ is only roughly a quarter of its total excitation energy of $20.40 \mathrm{eV}$. But the 'low' TKER rings share the problem that because of the high density of rings in this part of the image and their low intensity, no unimpeachable dependencies on the different wavelengths can be made out. As well, as it later comes apparent, some of them quite certainly derive from the same ionic molecular state.

One noticeable point is that the peak at $0.14 \mathrm{eV}$ has a nearly isotropic distribution, whereas all other peaks except for the free ion pair states are more or less parallel. As well is this peak slightly broader than the other ones, similar to the one at $2.16 \mathrm{eV}$ which is caused by VUV $+212.55 \mathrm{~nm}$. Hence it is the most likely candidate for a fragmentation mechanism apart from the others.

\section{Dissociation of the neutral $I_{2}$ and subsequent ionization by UV}

The main possibilities considered above will be put to test now for these peaks. Assumed dissociation of the neutral iodine molecule with VUV $(121.53 \mathrm{~nm})$ gives the possible designations of dissociation products which are shown in the table 14.3. All of the values agree reasonably well, but the results do not follow any obvious pattern but consist of a rather erratic choice of states.

Although the polarization check revealed that all of these peaks depend in the dissociative step on the VUV, dissociation of the neutral molecule by $212.55 \mathrm{~nm}$ was shortly considered. But even without the polarization check, this possibility can be discounted since it can only give TKER values in between 2.4 and $4.3 \mathrm{eV}$, which are far from the values here. 


\begin{tabular}{|c|c||c|c|}
\hline neutr. diss & & atomic levels & {$[\mathrm{eV}]$} \\
\hline \hline $\begin{array}{c}\text { exp. } \\
\text { value }[\mathrm{eV}]\end{array}$ & atomic levels & ${ }^{2} \mathrm{P}_{3 / 2}$ & ${ }^{2} \mathrm{P}_{1 / 2}$ \\
\hline 0.03 & $\left({ }^{3} \mathrm{P}_{1}\right) 6 \mathrm{~s}[1]_{3 / 2} 7.665$ & 0 & 0.943 \\
\hline 0.14 & $\left({ }^{3} \mathrm{P}_{2}\right) 5 \mathrm{~d}[4]_{7 / 2} 8.500$ & 0.159 & \\
& $\left({ }^{3} \mathrm{P}_{2}\right) 5 \mathrm{~d}[0]_{1 / 2} 8.507$ & 0.152 & \\
& $\left({ }^{1} \mathrm{D}_{2}\right) 6 \mathrm{~s}[2]_{5 / 2} 8.505$ & 0.155 & \\
& $\left({ }^{1} \mathrm{D}_{2}\right) 6 \mathrm{~s}[2]_{3 / 2} 8.499$ & 0.160 & \\
\hline 0.62 & $\left({ }^{3} \mathrm{P}_{2}\right) 6 \mathrm{p}[2]_{5 / 2} 8.047$ & 0.612 & \\
& $\left({ }^{3} \mathrm{P}_{2}\right) 6 \mathrm{p}[2]_{3 / 2} 8.058$ & 0.601 & \\
\hline 0.78 & $\left({ }^{3} \mathrm{P}_{2}\right) 6 \mathrm{~s}[2]_{3 / 2} 6.955$ & & 0.762 \\
& $\left({ }^{3} \mathrm{P}_{1}\right) 6 \mathrm{~s}[1]_{1 / 2} 7.834$ & 0.825 & \\
\hline
\end{tabular}

Table 14.3: Dissociation products from the neutral iodine molecule with $121.53 \mathrm{~nm}$. Combination of fragments are listed which give TKER near to the experimentally observed ones.

\section{Dissociation of the ionic molecule $\mathbf{I}_{2}^{+}$}

Possible designation from the dissociation of the ionized molecule are given in the tables 14.4, 14.5, 14.6 and 14.7. Since we know from the photoelectron image that both $\mathrm{X}^{2} \Pi_{g}$ and $\mathrm{A}^{2} \Pi_{u}$ states are produced, all of them are considered. But one has to keep in mind that the $\mathrm{A}^{2} \Pi_{u}$ states are produced by the absorption of two UV photons, see chapter 13, and that for the dissociation a third photon must be absorbed. This does not match the most probable combinations of absorbed photons as it was established in the section 14.2, thus this possibility is not very likely.

Inspection of the results shows that here the same problem as for the dissociation of the neutral molecule arises. A variety of states exist which match the measured TKER, but no pattern or symmetry comes apparent.

Dissociation of the ionic molecule by $212.55 \mathrm{~nm}$ can a be discounted for similar reasons as for the dissociation of the neutral by $212.55 \mathrm{~nm}$. Except for the $0.14 \mathrm{eV}$ peak, the dissociation by $212.55 \mathrm{~nm}$ does not yield any TKER which would match the results here.

\section{Ionization to a dissociative state}

The third possibility is the direct ionization to a dissociative ionic state. Here it is useful to have a look at experimental results from other groups. Tuckett et al. [3] performed a coincidence study of fluorescence and dissociation processes of electronic states of various halogens and interhalogens, among them 


\begin{tabular}{|c|c|c|c|}
\hline $\mathrm{I}_{2}^{+} \mathrm{X}^{2} \Pi_{3 / 2, g}$ & & $\mathrm{I}^{+}$states & {$[\mathrm{eV}]$} \\
\hline $\begin{array}{c}\text { exp. value } \\
{[\mathrm{eV}]}\end{array}$ & $\begin{array}{l}\text { atomic levels } \\
{[\mathrm{eV}]}\end{array}$ & $\begin{array}{c}{ }^{3} \mathrm{P}_{2} \\
0\end{array}$ & $\begin{array}{c}{ }^{3} \mathrm{P}_{0} \\
0.799\end{array}$ \\
\hline 0.62 & $\left({ }^{3} \mathrm{P}_{2}\right) 6 \mathrm{~s}[2]_{3 / 2} 6.955$ & $0.559(0.589)$ & \\
\hline 0.78 & $\left({ }^{3} \mathrm{P}_{2}\right) 6 \mathrm{~s}[2]_{5 / 2} \quad 6.774$ & $0.740(0.770)$ & \\
\hline & 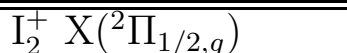 & $\overline{0}$ & $\overline{0.799}$ \\
\hline 0.03 & $\begin{array}{l}\left({ }^{3} \mathrm{P}_{2}\right) 6 \mathrm{p}[3]_{7 / 2} \\
\left({ }^{3} \mathrm{P}_{2}\right) 6 \mathrm{p}[3]_{5 / 2} \\
8.139\end{array}$ & $\begin{array}{l}0.016(0.044) \\
0.019(0.047)\end{array}$ & \\
\hline 0.14 & $\begin{array}{l}\left({ }^{3} \mathrm{P}_{2}\right) 6 \mathrm{p}[2]_{5 / 2} \\
\left({ }^{3} \mathrm{P}_{2}\right) 6 \mathrm{p}[2]_{3 / 2} 8.057\end{array}$ & $\begin{array}{c}0.110(0.139) \\
0.100(0.129))\end{array}$ & \\
\hline 0.62 & 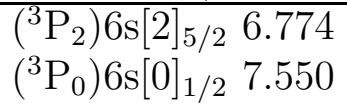 & $0.608(0.636)$ & $0.585(0.613)$ \\
\hline
\end{tabular}

Table 14.4: Dissociation products from the ground state $X\left({ }^{2} \Pi_{g}\right)$ ionic iodine molecule with $121.53 \mathrm{~nm}$ together with the corresponding TKER. The first TKER values give the nominal values for $I_{2}^{+}\left(v^{\prime}=0\right)$, the numbers in brackets denote the nominal TKER values for $I_{2}^{+}\left(v^{\prime}=1\right)$.

$\mathrm{I}_{2}^{+}$. The techniques they used are mass selected photoion-fluorescence photon coincidences counting (PIFCO) and TOF-MS by photoelectron-photoion coincidences (PEPICO). As the exciting wavelengths they used the emission from a rare-gas lamp, HeI $(584 \AA, 21.22 \mathrm{eV})$ and NeI (736-743 $\AA$ doublet, $16.85-16.67 \mathrm{eV})$.

They analyzed the mass peak profile of the PEPICO and found that the $\mathrm{I}^{+}$was produced in three different processes with the following total kinetic energy releases $0.14,0.97$ and $3.9 \mathrm{eV}$. The kinetic energy release was apparently the same for both excitation wavelengths, although a fourth process must exist since they found radiation from excited I atoms only at HeI excitation energy in the PIFCO experiment.

Since the processes were independent of the excitation radiation, it was postulated by the authors that direct ionization to dissociative states was the key mechanism. The two processes with low KER were assigned to the $\mathrm{B}^{2} \Sigma_{g}^{+}$state, dissociating to $\mathrm{I}\left({ }^{2} \mathrm{P}_{3 / 2}\right)+\mathrm{I}^{+}\left({ }^{3} \mathrm{P}_{2}\right)$ and $\mathrm{I}\left({ }^{2} \mathrm{P}_{3 / 2}\right)+\mathrm{I}^{+}\left({ }^{3} \mathrm{P}_{0,1}\right)$. This occurs in contrast to the findings of Eland [4], who claimed that the $\mathrm{B}^{2} \Sigma_{g}^{+}$ state only dissociates to ground state products.

The potential energy curves are illustrated in figure 14.6 , the $\mathrm{B}^{2} \Sigma_{g}^{+}$state is given by the upper red dashed line. The two black dashed lines represent two possible positions of dissociative Z state postulated by Tuckett et al. because of their experimental findings. This may well be the one perturbing the $\mathrm{B}^{2} \Sigma_{g}^{+}$state and causing it to dissociate to ground state products. However, 


\begin{tabular}{|c|c|c|c|c|c|}
\hline $\mathrm{I}_{2}^{+} \mathrm{A}^{2} \Pi_{3 / 2, u}$ & & $\mathrm{I}^{+}$states & {$[\mathrm{eV}]$} & & \\
\hline $\begin{array}{c}\text { exp. value } \\
{[\mathrm{eV}]}\end{array}$ & $\begin{array}{r}\text { atomic levels } \\
{[\mathrm{eV}]}\end{array}$ & $\begin{array}{c}{ }^{3} \mathrm{P}_{2} \\
0\end{array}$ & $\begin{array}{c}{ }^{3} \mathrm{P}_{0} \\
0.799\end{array}$ & $\begin{array}{c}{ }^{3} \mathrm{P}_{1} \\
0.879\end{array}$ & $\begin{array}{l}{ }^{1} \mathrm{D}_{2} \\
1.702\end{array}$ \\
\hline 0.03 & 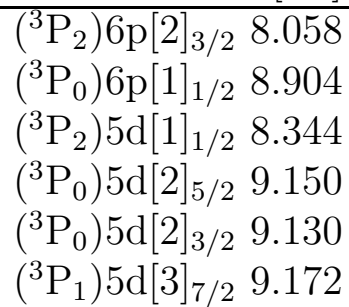 & $\begin{array}{l}(0.011) \\
0.035 \\
0.054 \\
0.012\end{array}$ & $\begin{array}{c}(0.057) \\
0.041\end{array}$ & & \\
\hline 0.14 & 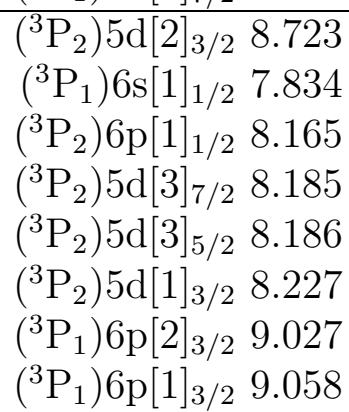 & $\begin{array}{l}0.158 \\
0.127\end{array}$ & $\begin{array}{l}0.200 \\
0.200 \\
0.158\end{array}$ & $\begin{array}{c}(0.202) \\
0.141 \\
0.121 \\
0.120\end{array}$ & \\
\hline 0.62 & $\begin{array}{cc}\left({ }^{3} \mathrm{P}_{2}\right) 6 \mathrm{p}[1]_{3 / 2} & 8.315 \\
\left({ }^{3} \mathrm{P}_{2}\right) 5 \mathrm{~d}[1]_{3 / 2} & 8.227 \\
\left({ }^{3} \mathrm{P}_{2}\right) 5 \mathrm{~d}[1]_{1 / 2} & 8.344 \\
\left({ }^{3} \mathrm{P}_{2}\right) 5 \mathrm{~d}[0]_{1 / 2} & 8.507 \\
\left({ }^{3} \mathrm{P}_{1}\right) 6 \mathrm{~s}[1]_{3 / 2} & 7.665 \\
\left({ }^{1} \mathrm{D}_{2}\right) 6 \mathrm{~s}[2]_{5 / 2} & 8.504\end{array}$ & $\begin{array}{c}(0.600) \\
(0.688) \\
(0.571) \\
0.677 \\
0.681\end{array}$ & 0.720 & 0.641 & \\
\hline 0.78 & $\begin{array}{cc}\left({ }^{3} \mathrm{P}_{2}\right) 6 \mathrm{p}[3]_{5 / 2} & 8.138 \\
\left({ }^{3} \mathrm{P}_{2}\right) 6 \mathrm{p}[1]_{1 / 2} & 8.165 \\
\left({ }^{3} \mathrm{P}_{0}\right) 6 \mathrm{~s}[0]_{1 / 2} & 7.550 \\
\left({ }^{3} \mathrm{P}_{1}\right) 6 \mathrm{~s}[1]_{3 / 2} & 7.665 \\
\left({ }^{3} \mathrm{P}_{1}\right) 5 \mathrm{~d}[4]_{9 / 2} & 8.397\end{array}$ & $\begin{array}{l}(0.776) \\
(0.749) \\
0.7886\end{array}$ & $\begin{array}{l}0.835 \\
0.720\end{array}$ & $\begin{array}{l}0.756 \\
0.641\end{array}$ & \\
\hline
\end{tabular}

Table 14.5: Dissociation products from the ionic $A^{2} \Pi_{3 / 2, u}$ iodine molecule with $121.53 \mathrm{~nm}$. The TKER values given without brackets include the vibrational excitation of the $I_{2}^{+}$determined in the photoelectron images, see section 13.2, the numbers in brackets denote the TKER if $v^{\prime}=0$ is assumed. Because the vibrational state most populated is quite high $\left(E_{v i b}=0.27 \mathrm{eV}\right)$, the values do not only change slightly as in the $X^{2} \Pi_{g}$ state case, which has approximately equal population of the $v^{\prime}=0$ and $V^{\prime}=1$ state, see table 14.4, but the energetically most likely fragments change to different combinations entirely.

even if it is not identical with the perturbing state which was proposed by 


\begin{tabular}{|c|r||c|c|c|c|}
\hline $\mathrm{I}_{2}^{+} \mathrm{A}^{2} \Pi_{1 / 2, u}$ & & $\mathrm{I}^{+}$states & {$[\mathrm{eV}]$} & & \\
\hline \hline $\begin{array}{c}\text { exp. value } \\
{[\mathrm{eV}]}\end{array}$ & atomic levels & ${ }^{3} \mathrm{P}_{2}$ & ${ }^{3} \mathrm{P}_{0}$ & ${ }^{3} \mathrm{P}_{1}$ & ${ }^{1} \mathrm{D}_{2}$ \\
& {$[\mathrm{eV}]$} & 0 & 0.799 & 0.879 & 1.702 \\
\hline 0.03 & $\left({ }^{3} \mathrm{P}_{0}\right) 6 \mathrm{p}[1]_{3 / 2} 8.924$ & $(0.821)$ & $(0.021)$ & & \\
& $\left({ }^{3} \mathrm{P}_{0}\right) 6 \mathrm{p}[1]_{1 / 2} 8.904$ & $(0.841)$ & $(0.041)$ & & \\
& $\left({ }^{1} \mathrm{D}_{2}\right) 6 \mathrm{p}[1]_{3 / 2} 9.722$ & $(0.0229$ & & & \\
& $\left({ }^{3} \mathrm{P}_{2}\right) 7 \mathrm{~d}[4]_{9 / 2} 9.802$ & 0.033 & & & \\
& $\left({ }^{3} \mathrm{P}_{2}\right) 7 \mathrm{~d}[4]_{7 / 2} 9.813$ & 0.021 & & & \\
& $\left({ }^{3} \mathrm{P}_{2}\right) 7 \mathrm{~d}[3]_{7 / 2} 9.783$ & 0.052 & & & \\
& $\left({ }^{3} \mathrm{P}_{2}\right) 7 \mathrm{~d}[3]_{5 / 2} 9.788$ & 0.047 & & & \\
& $\left({ }^{3} \mathrm{P}_{2}\right) 7 \mathrm{~d}[1]_{3 / 2} 9.799$ & 0.036 & & & \\
& $\left({ }^{3} \mathrm{P}_{0}\right) 6 \mathrm{p}[1]_{3 / 2} 8.924$ & & 0.111 & 0.032 & \\
& $\left({ }^{3} \mathrm{P}_{0}\right) 6 \mathrm{p}[1]_{1 / 2} 8.904$ & & 0.131 & 0.052 & \\
& $\left({ }^{3} \mathrm{P}_{1}\right) 6 \mathrm{p}[2]_{5 / 2} 8.993$ & 0.842 & 0.043 & & \\
& $\left({ }^{1} \mathrm{D}_{2}\right) 6 \mathrm{p}[3]_{7 / 2} 9.795$ & 0.039 & & & \\
\hline & $\left({ }^{3} \mathrm{P}_{2}\right) 5 \mathrm{~d}[2]_{3 / 2} 8.723$ & & & $(0.143)$ & \\
& $\left({ }^{3} \mathrm{P}_{1}\right) 6 \mathrm{~s}[1]_{1 / 2} 7.834$ & & & & $(0.208)$ \\
& $\left({ }^{3} \mathrm{P}_{0}\right) 6 \mathrm{p}[1]_{3 / 2} 8.924$ & & 0.111 & 0.032 & \\
& $\left({ }^{3} \mathrm{P}_{0}\right) 6 \mathrm{p}[1]_{1 / 2} 8.904$ & & 0.131 & 0.052 & \\
& $\left({ }^{1} \mathrm{D}_{2}\right) 6 \mathrm{p}[1]_{3 / 2} 9.722$ & 0.112 & & & \\
\hline
\end{tabular}

Table 14.6: Dissociation products from the ionic $A^{2} \Pi_{1 / 2, u}$ iodine molecule with $121.53 \mathrm{~nm}$. The TKER values given without brackets include the vibrational excitation of the $\mathrm{I}_{2}^{+}$determined in the photoelectron images, see section 13.2, the numbers in brackets denote the TKER if $v^{\prime}=0$ is assumed. Here the vibrational state most populated is not that high $\left(E_{v i b}=0.09 \mathrm{eV}\right)$ in comparison with the $A^{2} \Pi_{3 / 2, u}$ state, but because of the high density of states, also the energetically most likely fragments change to different combinations entirely.

Leach [5], as suggested in the schematic drawing of the potential, at least it is useful to illustrate the proposed interaction, since the $\mathrm{B}^{2} \Sigma_{g}^{+}$state primitively dissociates to ${ }^{1} \mathrm{D}_{2}+{ }^{2} \mathrm{P}_{3 / 2}$, which is known from $\mathrm{F}_{2}^{+}$calculations.

Tuckett et al. suggest 'It is not unlikely that dissociative states in this region exist since a large number of excited $\mathrm{I}_{2}^{+}$states can be formed by the two states of dissociation products $\mathrm{I}^{+}\left({ }^{3} \mathrm{P}\right)+\mathrm{I}\left({ }^{2} \mathrm{P}\right)$ and $\mathrm{I}^{+}\left({ }^{1} \mathrm{D}\right)+\mathrm{I}\left({ }^{2} \mathrm{P}\right)$. But a state of this kind can only be formed by direct ionization by relaxation of the oneelectron transition rule, which is e.g. possible via configuration interaction which is known to occur frequently in the case of halogen molecular ions.' [3]. The authors bring up that the photoelectron spectrum of such a state would 


\begin{tabular}{|c|c|c|c|c|c|}
\hline $\mathrm{I}_{2}^{+} \mathrm{A}^{2} \Pi_{1 / 2, u}$ & & $\mathrm{I}^{+}$states & {$[\mathrm{eV}]$} & & \\
\hline $\begin{array}{c}\text { exp. value } \\
{[\mathrm{eV}]}\end{array}$ & $\begin{array}{l}\text { atomic levels } \\
{[\mathrm{eV}]}\end{array}$ & $\begin{array}{c}{ }^{3} \mathrm{P}_{2} \\
0\end{array}$ & $\begin{array}{c}{ }^{3} \mathrm{P}_{0} \\
0.799\end{array}$ & $\begin{array}{c}{ }^{3} \mathrm{P}_{1} \\
0.879\end{array}$ & $\begin{array}{l}{ }^{1} \mathrm{D}_{2} \\
1.702\end{array}$ \\
\hline 0.62 & 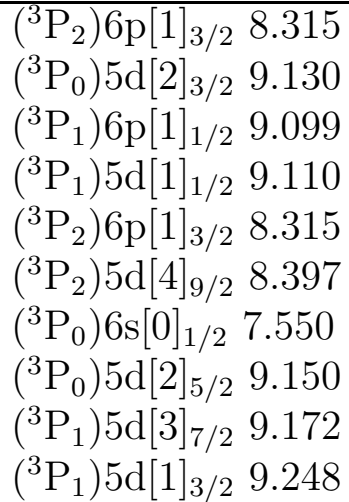 & $\begin{array}{l}(0.614) \\
(0.646) \\
(0.634) \\
\\
\\
0.685 \\
0.662 \\
0.587\end{array}$ & $\begin{array}{l}(0.630) \\
\\
0.720 \\
0.638\end{array}$ & 0.641 & 0.582 \\
\hline 0.78 & $\begin{array}{ll}\left({ }^{3} \mathrm{P}_{2}\right) 6 \mathrm{p}[2]_{3 / 2} & 8.058 \\
\left({ }^{3} \mathrm{P}_{2}\right) 6 \mathrm{p}[1]_{1 / 2} & 8.165 \\
\left({ }^{3} \mathrm{P}_{2}\right) 5 \mathrm{~d}[3]_{7 / 2} & 8.185 \\
\left({ }^{3} \mathrm{P}_{2}\right) 5 \mathrm{~d}[3]_{5 / 2} & 8.186 \\
5 \mathrm{~s} 5 \mathrm{p}^{6}{ }^{2} \mathrm{~S}_{1 / 2} & 9.065 \\
\left({ }^{3} \mathrm{P}_{2}\right) 6 \mathrm{p}[1]_{1 / 2} & 8.165 \\
\left({ }^{3} \mathrm{P}_{2}\right) 5 \mathrm{~d}[3]_{7 / 2} & 8.185 \\
\left({ }^{3} \mathrm{P}_{2}\right) 5 \mathrm{~d}[3]_{5 / 2} & 8.186 \\
\left({ }^{3} \mathrm{P}_{2}\right) 5 \mathrm{~d}[1]_{3 / 2} & 8.227 \\
\left({ }^{3} \mathrm{P}_{1}\right) 6 \mathrm{p}[2]_{3 / 2} & 9.027 \\
\left({ }^{3} \mathrm{P}_{1}\right) 6 \mathrm{p}[1]_{3 / 2} & 9.058\end{array}$ & $\begin{array}{l} \\
0.808 \\
0.777\end{array}$ & $\begin{array}{l}(0.780) \\
(0.760) \\
(0.760) \\
\\
0.870 \\
0.850 \\
0.850 \\
0.808\end{array}$ & $\begin{array}{l}(0.808) \\
(0.701) \\
(0.681) \\
(0.680) \\
\\
0.791 \\
0.771 \\
0.770 \\
0.729\end{array}$ & \\
\hline
\end{tabular}

Table 14.7: Continuation of table 14.6.

be very broad and therefore difficult to detect.

Because the authors already point out that their results in this region were definitely independent if either the $\mathrm{HeI}(21.22 \mathrm{eV})$ or NeI (16.85-16.67 eV) line was used and because in this experiment a similar total excitation energy of 20.40 or $16.03 \mathrm{eV}$ was used, it suggest itself that a relation to the results here exists. Now it is tempting to assume that the $0.14 \mathrm{eV}$ TKER peak here is the same as in the Tuckett experiment. But this I doubt because this particular peak is broader than the others, and it sports a low $\beta$-parameter of 0.19 , which is in contrast to all other rings.

These points hint more at the involvement of a metastable predissociative intermediate for the $0.14 \mathrm{eV}$ TKER peak.

Additionally, if we look out for both channels from the $\mathrm{B}^{2} \Sigma_{g}^{+}$state observed by Tuckett, the one at $0.97 \mathrm{eV}$ TKER is missing in this experiment. 


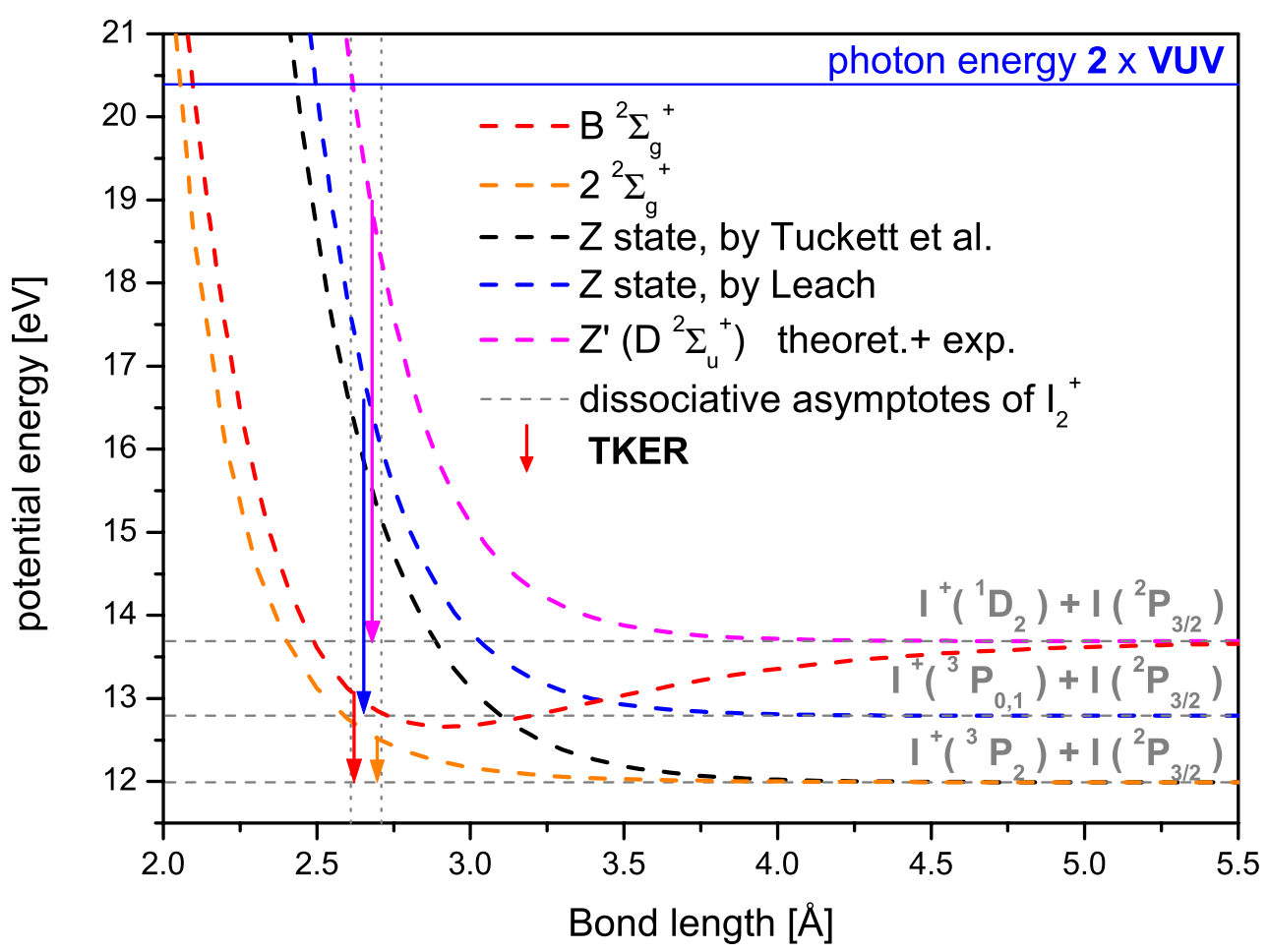

Figure 14.6: Schematic overview of the potentials of $I_{2}^{+}$involved in the dissociative ionization processes. This scheme is based on the results by Tuckett et al. [3] and Leach [5]. Ionic potentials are given in dashed lines, the dissociative asymptotes in light gray dashed lines and the Frank-Condon window by the light vertical gray dotted lines. As illustration some of the achieved TKER are represented by arrows going from the potentials to the appropriate dissociative asymptotes within the Frank-Condon window. These arrows are given in the colour of the corresponding potential curve. Included are the $B^{2} \Sigma_{g}^{+}$state, which has been observed in photoelectron spectra, its lower $2^{2} \Sigma_{g}^{+}$state (see text), both possible position of the $Z$ state and the proposed $Z$ ' state. The latter states were both found in the experiments by Tuckett et al. [3]. Please note that the $B^{2} \Sigma_{g}^{+}$state can only dissociate to ground state products as indicated with the red arrow by interaction with another state. This interaction could here be an interaction with the $Z$ state, but this should be understood as an illustrative example of the principle and not as a concrete assignment.

But here a channel with a TKER of $0.78 \mathrm{eV}$ exists, which is $0.19 \mathrm{eV}$ lower. If we look now for a channel $0.19 \mathrm{eV}$ lower than the $0.14 \mathrm{eV}$, of course there is none, but the channel at $0.03 \mathrm{eV}$ is with $0.11 \mathrm{eV}$ lower in the same order, 
as well does it exhibit a similar $\beta$-parameter (0.92 and 1.23). Therefore I tentatively assign these two channels at 0.03 and $0.78 \mathrm{eV}$ to the dissociative $\mathrm{B}^{2} \Sigma_{g}^{+}$state. Because the $\mathrm{B}^{2} \Sigma_{g}^{+}$state can be reached with either two VUV photons or a UV+VUV combination, this dependency cannot be further clarified. From the intensity check, both seemed to depend on two VUV photons, although in the Lyman- $\alpha(\mathrm{D}) /(\mathrm{H})$ comparison, the $0.78 \mathrm{eV}$ TKER peak exhibited a comparatively low factor $(11.5$ instead of $\approx 16)$. From these findings it can still be possible that the process has a small contribution from a VUV+UV combination. But I assume that it is negligible small. Thus the ratio of the two channels was determined as $0.32: 1$ for ${ }^{2} \mathrm{P}_{3 / 2}+{ }^{3} \mathrm{P}_{0,1}$ and ${ }^{2} \mathrm{P}_{3 / 2}+{ }^{3} \mathrm{P}_{2}$ respectively.

Together with the known threshold energy to produce ${ }^{2} \mathrm{P}_{3 / 2}+{ }^{3} \mathrm{P}_{2}$ $(11.995 \mathrm{eV})$ and ${ }^{2} \mathrm{P}_{3 / 2}+{ }^{3} \mathrm{P}_{0}(12.794 \mathrm{eV})$, the transition energy to the $\mathrm{B}^{2} \Sigma_{g}^{+}$ state was determined as 12.77 and 12.82 respectively, which is in very good agreement with results obtained from conventional photoelectron spectroscopy which give the onset of the ionization to the $\mathrm{B}^{2} \Sigma_{g}^{+}$state as 12.7 and 12.79 respectively [6,7], although all of them obtained $12.95 \mathrm{eV}$ as the maximum intensity value, which is more in line with the results of 0.14 and $0.97 \mathrm{eV}$ from Tuckett et al. The only possible explanation why here the results should be different I can think of is that because here it is a two photon process, the absorption process is different and favours a slightly different Frank-Condon window.

The experiments from Unny et al. with multiphoton absorption in the wavelength range $556-575 \mathrm{~nm}$ at intermediate intensities gave TKER peaked at $0.62,0.98$ and $1.98 \mathrm{eV}$. As well a ring around $0.1 \mathrm{eV}$ appeared at two wavelengths and they observed a rather pronounced central feature which peaked at zero but had a parallel $\beta$-parameter for slightly higher TKER. The 0.1 and $0.98 \mathrm{eV}$ features could be the ones from dissociation of the $\mathrm{B}^{2} \Sigma_{g}^{+}$ state, which is further proof that dissociative ionization is the dominant channel in iodine in this energy regime .

\section{$0.62 \mathrm{eV}$ peak}

In this experiment a feature at $0.62 \mathrm{eV}$ was found, but not in all spectra. Actually it poses a small problem. If the free ion pair production is strong, this peak is swallowed in the base of the free ion pair peak at $0.41 \mathrm{eV}$. From the above discussion of relative intensities it is only evident that the dissociating step does depend on VUV, but since the total intensity is rather low, and only few images exhibit this feature, it is impossible to decide if it depends 
on two VUV photons or one UV and one VUV. It seems rather broad in this experiment, which is actually due more to its appearance as a flat plateau without a pronounced maximum, and lies in a crowded part of the image. An examination of the raw images shows that it seems more to be a continuation of the $0.78 \mathrm{eV}$ peak, despite its smaller $\beta$-parameter, which is separated from the main peak with a small 'ring' of no intensity.

Unny et al. also found a peak at $0.62 \mathrm{eV}$, but unfortunately the authors do not discuss the nature of their results. The main idea is that they are apparently wavelength independent and are probably produced by six or more photons in the $556-575 \mathrm{~nm}$ range, but no discussion of possible states follows.

If ground state products are assumed here, the state energy for the $0.62 \mathrm{eV}$ peak would be at $12.62 \mathrm{eV}$, just below the $\mathrm{B}^{2} \Sigma_{g}^{+}$state, or to be more precise, in the lower limit of the ionization energies given in various publication. Here, one should remember that Leach [5] postulated that the $\mathrm{B}^{2} \Sigma_{g}^{+}$state observed in photoelectron spectra is in fact a higher state of two $\Sigma$-states, deriving from the 2331 (lower) and 1440 (higher) configuration, see figure 14.6. The lower one was not yet observed in a photoelectron spectrum. When discussing results from photodissociation experiments of the halogen cations $\mathrm{Cl}_{2}^{+}, \mathrm{Br}_{2}^{+}$ and $\mathrm{I}_{2}^{+}$by McLoughlin et al. [8], he gives the well depth of the upper $\mathrm{B}^{2} \Sigma_{g}^{+}$as $1.03 \mathrm{eV}$ dissociating to ${ }^{1} \mathrm{D}_{2}+{ }^{1} \mathrm{P}_{u}$, which can only dissociate to ground state dissociation products by interaction with either a postulated perturbing state or the proclaimed lower $\Sigma$-state, called $2^{2} \Sigma_{g}^{+}$. It can be speculated that in a vertical transition from the neutral ground state, the lower state is populated as well and that the difference in TKER represents the difference in vertical transition energy, see fig. 14.6.

Additionally, de Jong et al [9] proposed in their relativistic calculations the positions of other $1 / 2_{g}$ states based on the 2331 configuration or a mixture of the 2331 and 1440 configuration. These are given as $0.09 \mathrm{eV}$ below and $0.12 \mathrm{eV}$ above the $\mathrm{B}^{2} \Sigma_{g}^{+}$state respectively, which is of course only an indication since these states have not yet been observed experimentally. The lower one may be identical to the proposed lower $2^{2} \Sigma_{g}^{+}$state. Because in this work a two photon scheme was employed, this configuration may contribute more than in a one-photon excitation scheme and results in the $0.62 \mathrm{eV}$ peak.

One thing is still to consider. Although the maximum of this plateaulike distribution can be made out at the TKER value at $0.62 \mathrm{eV}$, it is more a rough idea where the real maximum might be.

It was already discussed that it seems a bit like the continuation of the $0.78 \mathrm{eV}$ peak to lower TKER values. Altogether it is then similar to the wave- 


\begin{tabular}{|c|l||c|c|c|}
\hline $\begin{array}{c}3 \text { body decay } \\
2 \times \text { VUV }\end{array}$ & & $\mathrm{I}^{+}$states & {$[\mathrm{eV}]$} & \\
\hline \hline $\begin{array}{c}\text { exp. values } \\
{[\mathrm{eV}]}\end{array}$ & atomic levels & ${ }^{3} \mathrm{P}_{2}$ & ${ }^{3} \mathrm{P}_{0}$ & ${ }^{3} \mathrm{P}_{1}$ \\
& {$[\mathrm{eV}]$} & 0 & 0.799 & 0.879 \\
\hline 0.03 & $\left({ }^{3} \mathrm{P}_{2}\right) 6 \mathrm{p}[1]_{3 / 2} 8.315$ & 0.095 & & \\
& $\left({ }^{3} \mathrm{P}_{2}\right) 5 \mathrm{~d}[4]_{9 / 2} 8.397$ & 0.013 & & \\
& $\left({ }^{3} \mathrm{P}_{2}\right) 5 \mathrm{~d}[1]_{1 / 2} 8.344$ & 0.066 & & \\
& $\left({ }^{3} \mathrm{P}_{0}\right) 6 \mathrm{~s}[0]_{1 / 2} 7.550$ & 0.859 & 0.060 & \\
\hline 0.14 & $\left({ }^{3} \mathrm{P}_{2}\right) 6 \mathrm{p}[3]_{7 / 2} 8.142$ & 0.268 & & \\
& $\left({ }^{3} \mathrm{P}_{2}\right) 6 \mathrm{p}[3]_{5 / 2} 8.139$ & 0.271 & & \\
& $\left({ }^{3} \mathrm{P}_{2}\right) 6 \mathrm{p}[1]_{1 / 2} 8.165$ & 0.244 & & \\
& $\left({ }^{3} \mathrm{P}_{2}\right) 5 \mathrm{~d}[3]_{7 / 2} 8.185$ & 0.225 & & \\
& $\left({ }^{3} \mathrm{P}_{2}\right) 5 \mathrm{~d}[3]_{5 / 2} 8.186$ & 0.224 & & \\
& $\left({ }^{3} \mathrm{P}_{2}\right) 5 \mathrm{~d}[1]_{3 / 2} 8.227$ & 0.183 & & \\
\hline 0.62 & $\left({ }^{3} \mathrm{P}_{1}\right) 6 \mathrm{~s}[1]_{1 / 2} 7.834$ & 0.575 & & \\
& $\left({ }^{3} \mathrm{P}_{2}\right) 6 \mathrm{~s}[2]_{3 / 2} 6.955$ & & 0.655 & \\
\hline 0.78 & $\left({ }^{3} \mathrm{P}_{2}\right) 6 \mathrm{~s}[2]_{5 / 2} 6.774$ & & 0.836 & 0.757 \\
& $\left({ }^{3} \mathrm{P}_{1}\right) 6 \mathrm{~s}[1]_{3 / 2} 7.665$ & 0.745 & & \\
& $\left({ }^{3} \mathrm{P}_{0}\right) 6 \mathrm{~s}[0]_{1 / 2} 7.550$ & 0.859 & 0.060 & \\
\hline
\end{tabular}

Table 14.8: Possible three body decay products from two VUV photons for the low TKER values.

length dependent distributions depending on VUV and 365 / $266 \mathrm{~nm}$. These are attributed to a three body process, where the electron takes away a broad distribution of energy, with a maximum of total kinetic energy release if the kinetic energy of the electron is zero. Those distributions peak near their maximum values. A look at table 14.8 shows that the energetically most fitting dissociation product with two VUV photons would be $\mathrm{I}\left(\left({ }^{3} \mathrm{P}_{2}\right) 6 \mathrm{~s}[2]_{5 / 2}\right)+$ $\mathrm{I}^{+}\left({ }^{3} \mathrm{P}_{0}\right)$ with a maximum TKER of $0.836 \mathrm{eV}$, if a similar behaviour as in the $\mathrm{UV}+\mathrm{VUV}$ experiments is assumed. Because this is a rather erratic choice this possibility remains unconvincing.

For the sake of completeness table 14.8 also contains possible TKER and fragment assignments for all other low TKER observed if three body decay is assumed. But because these other peaks do not show the typical broad distribution of a three body decay and since the values do not match convincingly the observed TKER, I believe that three body decay can be excluded as a possible mechanism for these states.

A three body process initiated with $212.55 \mathrm{~nm}+\mathrm{VUV}$ does not give matching TKER values either, see table 14.13. 


\section{$0.14 \mathrm{eV}$ peak}

The ring at $0.14 \mathrm{eV}$ TKER is qualitatively not only very similar to the wavelength independent rings observed in the UV+VUV experiment, with its $\beta$-parameter indicating a nearly isotropic distribution, but also it seem to depend on a VUV+UV photon combination with a total excitation energy of $16.03 \mathrm{eV}$. Those wavelength independent rings were assigned to the dissociation of the neutral molecule by VUV radiation, with a subsequent ionization by the UV light, because their TKER were independent of the UV wavelength. All three rings in that experiment had a nearly isotropic distribution. The smallest ring corresponded to a TKER of $0.16 \mathrm{eV}$, which suggests that the one observed here is identical to it, especially since no exactly matching asymptotes for this value can be found, compare table 14.3. But if the two experiments are directly compared, i.e. with images from the same day under the same conditions for the electrostatic lens, the two peaks are very obviously not in the same position. That only one of those three peaks reappears can still be explained by the position of the other two. Their TKER are 0.48 and $0.29 \mathrm{eV}$. Since they must be comparable in intensity to the $0.14 \mathrm{eV}$ peak, they may be to low in intensity to be distinguishable from the free ion pair peak at $0.41 \mathrm{eV}$.

Still, the interpretation of the $0.14 \mathrm{eV}$ peak as dissociation of the neutral molecule remains doubtful, although I believe it points in the right direction. The dissociation to high lying Rydberg states of the neutral with $20.40 \mathrm{eV}$ excitation energy is supported by the fluorescence from neutral fragments Tuckett et al [3] found in their experiments at HeI excitation wavelength.

Another option, which should be mentioned is the dissociation of the ionic first spin orbit excited state $\mathrm{X}^{2} \Pi_{1 / 2, g}$ state to ${ }^{2} \mathrm{P}_{3 / 2}+{ }^{1} \mathrm{~S}_{0}$. This gives a nominal TKER of $0.132 \mathrm{eV}$. This may be a possibility, but due to its arbitrary choice of fragmentation products and the lack of observation of other dissociation processes from the ionic molecule it remains a mere possibility.

\section{$0.03 \mathrm{eV}$ peak}

The feature at $0.03 \mathrm{eV}$ resembles closely the central feature observed by Unny et al. [10] in VMI experiment where dissociative ionization was studied with multiphoton absorption between $556-575 \mathrm{~nm}$ in the intermediate intensity regime. They observed a central feature with an isotropic distribution near zero kinetic energy which goes to a distinct parallel transition with increasing kinetic energy. A closer look at the published images shows a rather 
unstructured blob smeared out in a parallel direction. This looks similar to the feature found in this work which peaks at $0.03 \mathrm{eV}$ TKER, but in contrast to the experiments by Unny et al., the feature could be resolved in some images and a closer inspection reveals that it is actually made up of a central dot and a sharp ring displaying a parallel transition at $0.03 \mathrm{eV}$ TKER which are clearly removed from each other. But although the feature at $0.03 \mathrm{eV}$ was already assigned to the dissociation of $\mathrm{B}^{2} \Sigma_{g}^{+}$to $\left.\mathrm{I}^{2} \mathrm{P}_{3 / 2}\right)+\mathrm{I}^{+}\left({ }^{3} \mathrm{P}_{0}\right)$, the resemblance is close enough to discuss its implications.

The authors explain that the only possible mechanism to produce this kind of shapeless feature they found is a three body process where nearly all of the energy is taken away by the electron. They propose transition to doubly excited high lying Rydberg state above the ionizing threshold: 'Competition between dissociation from the neutral state and autoionization to a dissociative ionic state produces a continuous distribution of ionic kinetic energies. If the ionic curve is flat or weakly bound in the Frank-Condon region, most or all of the kinetic energy release comes from repulsion of the upper, neutral curve.' They assume that here 6 photons are used, which gives roughly $13.3 \mathrm{eV}$ excitation energy since the feature was most prominent at wavelengths around $558 \mathrm{~nm}$.

Their explanation that here three body processes are dominant in contrast to the behaviour in the weak field is that excitation of more than one electron is much easier. The dependence of the $\beta$-parameter of the central peak is explained as follows: 'The isotropic central is possibly produced by molecules that ionize promptly and hence dissociate slowly on a weakly bound surface,.... The molecules that undergo slow autoionization recoil more rapidly on the neutral surface and therefore retain some memory of the initial alignment.' The authors also contemplate the possibility of alignment by the laserfield as an alternative.

If compared to the UV+VUV experiments described in section 15, this kind of behaviour seems rather surprising. All other three-body-processes observed in this work in the excitation regime of $16.03 \mathrm{eV}$ to $13.60 \mathrm{eV}$ start at the maximum energy if the electron takes away no energy at all and are all very broad, usually they peak at a TKER approximately $0.2 \mathrm{eV}$ lower than the maximal TKER.

The $0.03 \mathrm{eV}$ feature in this work was presumed to have an excitation energy of $20.40 \mathrm{eV}$ induced by two VUV photons. If the three body mechanism as described by the authors holds true, the neutral upper state around $13.3 \mathrm{eV}$ cannot be reached by an excitation energy of $20.40 \mathrm{eV}$ with a resonant step. But the lower lying ionic state should be reachable by direct ionization to this state, actually their proposed excitation energy of $13.3 \mathrm{eV}$ is so near to the transition energy to the $\mathrm{B}^{2} \Sigma_{g}^{+}$state that it suggests itself as the final 
dissociating state. But even the other proposed states in the vicinity can be reached as mentioned in the discussion of the $0.62 \mathrm{eV}$ peak because of the two photon process and can serve as the final dissociating state. The lower $\Sigma$-state $2^{2} \Sigma_{g}^{+}$for example is based on the 2331 configuration instead of the 1440 configuration which dominates the upper $\mathrm{B}^{2} \Sigma_{g}^{+}$state.

But the authors claim that prompt ionization to the ionic surface results in slow dissociation with nearly no kinetic energy and an isotropic angular distribution. This behaviour should be the same if this surface is reached in direct ionization. That only the anisotropic part is reproduced here but not the smeared out isotropic part at such a low energy as in the feature of Unny et al. indicates that no dissociation process via the ionic curve only was present in this work, thus no congruence of the two models can be established.

This implies that these are despite their similar appearance totally independent processes. But assuming that the same process is the basis here, obviously the mechanism cannot be fully understood. It can also be envisaged that the feature observed by Unny et al. is actually exactly the same as in this work but simply more photons were absorbed than they anticipated resulting in a similar excitation energy as in this work.

\subsubsection{Peaks with high TKER}

For the peaks with high TKER (3.91, 4.88 and $5.77 \mathrm{eV})$ the first possibility, which is dissociation of the neutral molecule can be quickly discounted, because no states of the neutral atom exist which generate matching TKER.

\section{Dissociation of the ionic ground state}

The mass spectrum shows that $\mathrm{I}_{2}^{+}$is produced in great abundance and from the photoelectron spectrum it is known that the $\mathrm{X}^{2} \Pi_{g}$ states and the $\mathrm{A}^{2} \Pi_{u}$ states are produced, as well as a fifth peak which is tentatively assigned as $a^{4} \Sigma_{u}^{-}$. But because the $\mathrm{A}^{2} \Pi_{u}$ and the $a^{4} \Sigma_{u}^{-}$state are initiated with two UV photons and the peaks here definitely derive from two VUV photons, only dissociation of the $\mathrm{X}^{2} \Pi_{g}$ states is considered. Only the $\mathrm{X}^{2} \Pi_{3 / 2, g}$ state give TKER values in the appropriate region. These dissociation products from the $\mathrm{X}^{2} \Pi_{3 / 2, g}$ state with $121.53 \mathrm{~nm}$ wavelength are shown in table 14.9 .

It is obviously possible to match these features to dissociation processes from the ionic molecule, although the process $\mathrm{I}\left({ }^{2} \mathrm{P}_{1 / 2}\right)+\mathrm{I}^{+}\left({ }^{3} \mathrm{P}_{0}\right)$ is forbidden by transition rules. Because it is a parallel transition which indicates $\Delta \mathrm{J}=0$, the resulting $J$ should be $3 / 2$. But these fragmentation products only allows a total J-value of $1 / 2$ for the dissociative curve. This still leaves two possi- 


\begin{tabular}{|l||c|c|c|c|}
\hline $\mathrm{I}_{2}^{+} \mathrm{X}^{2} \Pi_{3 / 2, g}$ & & & & \\
\hline \hline $\mathrm{I}^{+}$states & ${ }^{3} \mathrm{P}_{0}$ & ${ }^{3} \mathrm{P}_{1}$ & ${ }^{1} \mathrm{D}_{2}$ & ${ }^{1} \mathrm{~S}_{0}$ \\
{$[\mathrm{eV}]$} & 0.799 & 0.879 & 1.702 & 3.658 \\
\hline I states $[\mathrm{eV}]$ & & & & \\
${ }^{2} \mathrm{P}_{3 / 2} 0$ & & & $5.812(5.841)$ & $3.856(3.886)$ \\
${ }^{2} \mathrm{P}_{1 / 2} 0.943$ & $5.772(5.801)$ & $5.692(5.722)$ & $4.869(4.898)$ & \\
\hline
\end{tabular}

Table 14.9: Dissociation products from the ionic ground state $X^{2} \Pi_{3 / 2, g}$ with $121.53 \mathrm{~nm}$. The first TKER values give the nominal values for $I_{2}^{+}\left(v^{\prime}=0\right)$, the numbers in brackets denote the nominal TKER values for $I_{2}^{+}\left(v^{\prime}=1\right)$.

ble states for the $5.765 \mathrm{eV}$ TKER value, although the peak is not noticeably broadened compared to the other two rings.

As well it is problematic that at these high energies, which can be reached from the ionic ground state $\mathrm{X}^{2} \Pi_{g}$ with a UV or VUV photon in a vertical transition, no reliable data on ionic molecular states exists. There are relativistic calculations up to couple of eVs $[9,11]$ above the first ionization limit and experimental data on repulsive states, but no coherent and generally accepted picture exists so far concerning ionic states above the $\mathrm{B}^{2} \Sigma_{g}^{+}$state.

\section{Ionization to dissociative states}

But as was already discussed in the low TKER section, Tuckett et al. observed a peak at $3.9 \mathrm{eV}$ TKER in their experiment, which was independent of the excitation wavelength, HeI or NeI. Later, Leach [5], who was one of the authors of the initial paper, claimed that the high energy peak was actually at $5.9 \mathrm{eV}$ TKER for the HeI $(21.22 \mathrm{eV})$ excitation wavelength. These values are nearly identical to the 3.91 and $5.77 \mathrm{eV}$ values observed here, which implies that again direct ionization to dissociative state occurs, which should be wavelength independent within a certain range.

Their interpretation of these peaks is that two different dissociative states are involved. For the $3.9 \mathrm{eV}$ state they introduced a new dissociative state $\mathrm{Z}$ which unfortunately does not match any calculated state. This state would have a minimum vertical transition energy of $11.995+3.9 \mathrm{eV}=15.9 \mathrm{eV}$ if ground state products are assumed. In the illustrations of the ionic potentials in the left panel of fig. 14.7 this $\mathrm{Z}$ state is represented by the dashed green line, its vertical transition energy by the solid green line. The $5.9 \mathrm{eV}$ TKER should belong to the production of $\mathrm{I}^{+}\left({ }^{1} \mathrm{D}_{g}\right)+\mathrm{I}\left({ }^{1} \mathrm{P}_{u}\right)$, analogous to similar findings in $\mathrm{F}_{2}^{+}$, and the resulting dissociative state $\mathrm{Z}$ ' state can be assigned as $\mathrm{D}^{2} \Sigma_{u}^{+}$, which was calculated to be at $19.73 \mathrm{eV}$ by Dyke et al. 
[11]. The transition energy obtained with the experimental result here is then $19.46 \mathrm{eV}$. This potential is shown in figure 14.7 by the pink dashed line.

Now where does the $4.88 \mathrm{eV}$ TKER fit in? Since the $4.88 \mathrm{eV}$ ring I observed has effectively the same $\beta$-parameter and peak width as the $3.91 \mathrm{eV}$ ring, a first assumption is that it comes from the same state. The highly parallel transition suggests a direct transition to the dissociative or weakly bound state, which may then dissociate directly or via interaction with a second or third state.

If this is the case, it means that the $3.91 \mathrm{eV}$ channel does not dissociate to ground state dissociation products but to either of the combination of $\mathrm{I}^{+}\left({ }^{3} \mathrm{P}_{0}\right)+\mathrm{I}\left({ }^{2} \mathrm{P}_{3 / 2}\right), \mathrm{I}^{+}\left({ }^{3} \mathrm{P}_{1}\right)+\mathrm{I}\left({ }^{1} \mathrm{P}_{3 / 2}\right), \mathrm{I}^{+}\left({ }^{3} \mathrm{P}_{2}\right)+\mathrm{I}\left({ }^{1} \mathrm{P}_{1 / 2}\right)$ and the $4.88 \mathrm{e} \mathrm{V}$ peak must belong to ground state dissociation products $\mathrm{I}^{+}\left({ }^{3} \mathrm{P}_{2}\right)+\mathrm{I}\left({ }^{2} \mathrm{P}_{3 / 2}\right)$. This is only possible by interaction with another state dissociating to ground state products. This model is shown in the right panel of figure 14.7. Here the green dashed curve leads to excited fragments and the cyan dashed line represents schematically the interaction to the ground state fragments.

From the average ratio of the two peaks which is 0.97:1 it can be deduced that the dissociation to excited products, which exact channel or channels are however involved, and to ground state products has equal probability. The transition energy of the $\mathrm{Z}$ state is in this picture $11.995 \mathrm{eV}+4.88 \mathrm{eV}$ which makes a total energy of $16.88 \mathrm{eV}$ (solid green line in right panel) which is basically isoenergetic with the NeI radiation used in the Tuckett experiment.

Leach already points out the possibility that the $3.9 \mathrm{eV}$ process may belong to a state isoenergetic with the Ne I radiation and dissociating to spin orbit excited products. This state is best assigned to the $1 \pi_{u}^{4} 1 \pi_{g}^{2} 2 \sigma_{u}$ configuration which would require either a two photon transition or configuration interaction to be reached with one photon. Here, two photons are available which explains that this process has a higher contribution here than in the Tuckett experiment compared to the peaks at low TKER, but it is interesting that they only saw the $3.9 \mathrm{eV}$ channel.

The initial experiments by Tuckett et al. were performed as a comparison of the dissociation dynamics of the halogens $\mathrm{Cl}_{2}, \mathrm{Br}_{2}$ and $\mathrm{I}_{2}$. Leach points out that because no equivalent process were observed in the PEPICO experiments on the other halogens, the process initiated with $\mathrm{NeI}$ at $16.85 \mathrm{eV}$ might be specific to the $\mathrm{I}_{2}^{+}$case or that the corresponding state lies higher for $\mathrm{Cl}_{2}^{+}$ and $\mathrm{Br}_{2}^{+}$therefore they are not observed. This supports the conclusion that the process is exactly at the NeI wavelength and thus give spin orbit excited asymptotes $\mathrm{I}^{+}\left({ }^{3} \mathrm{P}_{0,1}\right)+\mathrm{I}\left({ }^{2} \mathrm{P}_{3 / 2}\right)$ with 16.71 and $16.79 \mathrm{eV}$ transition energy for the dissociative $\mathrm{Z}$ state. 


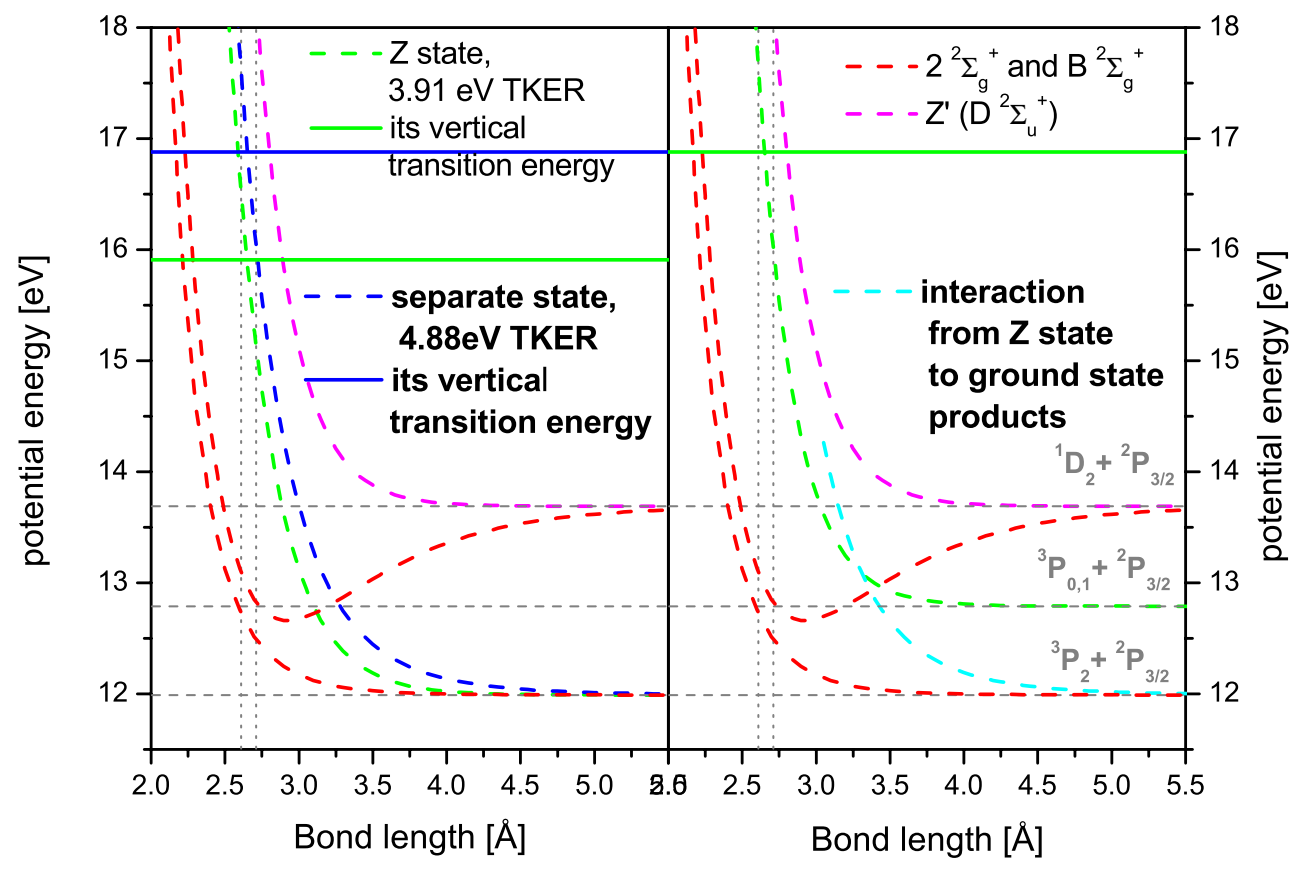

Figure 14.7: Modified schematic overview of the potentials of $\mathrm{I}_{2}^{+}$involved in the dissociative ionization processes. Ionic potentials are given in dashed lines, the dissociative asymptotes in light gray dashed lines and the FrankCondon window by the light vertical gray dotted lines. Two possibilities are shown where the results of Tuckett et al. [3] and Leach [5] are modified to match the results of this work. In the left panel a separate state was added to the $Z$ state introduced by Tuckett et al. to allow for the $4.88 \mathrm{eV}$ TKER, and in the right panel an interactive state was introduced to allow the $Z$ state by Leach to dissociate to ground state products. This would result in $3.91 \mathrm{eV}$ TKER for excited products and $4.88 \mathrm{eV}$ for ground state products. Additionally, the vertical transition energies resulting from the two possible discussed positions of the $Z$ state are indicated with the horizontal green lines, whereas the transition energy to the separate state leading to $4.88 \mathrm{eV}$ is shown as a blue solid line.

The $4.88 \mathrm{eV}$ channel can also originate in the same Z' state as the $5.9 \mathrm{eV}$ channel which was only observed by Tuckett at the higher excitation energy. If the $4.88 \mathrm{eV}$ channel comes from the same state, which is supposed to produce $\mathrm{I}^{+}\left({ }^{1} \mathrm{D}_{2}\right)+\mathrm{I}\left({ }^{2} \mathrm{P}_{u}\right)$ analogous to $\mathrm{F}_{2}^{+}$experiments, it is of course energetically possible that the $4.88 \mathrm{eV}$ channel comes from dissociation to $\mathrm{I}^{+}\left({ }^{1} \mathrm{D}_{2}\right)+\mathrm{I}\left({ }^{2} \mathrm{P}_{1 / 2}\right)$ instead of $\mathrm{I}^{+}\left({ }^{1} \mathrm{D}_{2}\right)+\mathrm{I}\left({ }^{2} \mathrm{P}_{3 / 2}\right)$. This gives then a transition energy of $19.52 \mathrm{eV}$. Then again an interactive state must exist which allows 
for the different dissociation channels.

The ratio of these two channels is determined from the integrated signal as $0.41: 1$ for the 5.77 and $4.88 \mathrm{eV}$ TKER respectively, which would make the ground state product channel less populated than the one leading to spin orbit excited products.

Also conceivable is that the $4.88 \mathrm{eV}$ state only has by chance the appropriate energy gap to the other peaks that infers production of a both ground state and spin orbit excited asymptote combinations. There may exists a repulsive potential in this region that can give the $4.88 \mathrm{eV}$ peak, which would indicate a minimum state energy of $11.995+4.88 \mathrm{eV}$ as discussed above, but is independent of the $3.91 \mathrm{eV}$ peak which could still come from a state with a minimum of $11.995+3.91 \mathrm{eV}=15.905 \mathrm{eV}$ transition energy. In fig. 14.7, left panel, this is illustrated with the blue dashed line going to ground state products giving $4.88 \mathrm{eV}$ TKER and the green dashed line giving $3.91 \mathrm{eV}$ TKER. The green solid line indicates then the minimum vertical transition energy to the $\mathrm{Z}$ state. If three independent states/mechanisms for the three TKER values are assumed, their relative yields are $0.41,0.42$ and 0.17 for 3.91, 4.88 and $5.77 \mathrm{eV}$ TKER respectively by excitation with two $121.53 \mathrm{~nm}$ photons.

Of course the $4.88+11.995 \mathrm{eV}$ transition energy is the limit for ground state products, this limit is shown by the blue solid line in the left panel. If excited products occur the state will be higher by the appropriate energy. If it is an independent state the reason that the $4.88 \mathrm{eV}$ TKER was not seen in the Tuckett experiment could be that the state is not reachable in a one photon transition.

Another explanation why it was not seen at all in the Tuckett experiments is that it was simply overlooked due to poor resolution. All the TKER were determined from the analysis of the mass peak profile, and apparently the $5.9 \mathrm{eV}$ peak was only recognized some years later.

\section{Three body decay}

Because in the UV+VUV case three body decay occurred, this option is here also considered. In the table the maximal values for the three body decay with two $121.53 \mathrm{~nm}$ photons are given. Those are where the electron takes away no energy at all. This means that the neutral molecule is ionized and dissociated in one step and all excess energy is released as kinetic energy of the iodine.

If only the TKER is considered, this process is also possible. As an advantage over the considered dissociation of the ionic molecule, only one possible designation exists for each peak. 


\begin{tabular}{|l||c|c|}
\hline $\begin{array}{l}3 \text { body decay } \\
2 \times \text { VUV }\end{array}$ & & \\
\hline \hline $\mathrm{I}^{+}$states & ${ }^{1} \mathrm{D}_{2}$ & ${ }^{1} \mathrm{~S}_{0}$ \\
{$[\mathrm{eV}]$} & 1.702 & 3.658 \\
\hline $\mathrm{I}$ states $[\mathrm{eV}]$ & & \\
${ }^{2} \mathrm{P}_{3 / 2} 0$ & & 4.750 \\
${ }^{2} \mathrm{P}_{1 / 2} 0.943$ & 5.765 & 3.809 \\
\hline
\end{tabular}

Table 14.10: Possible three body decay products from two VUV photons for the high TKER peaks.

But firstly, the three body process was observed in the range of $14-16 \mathrm{eV}$ total excitation energy. Secondly, the peaks are all very sharp, which is in disaccord with the picture of an electron which can take away a variable amount of kinetic energy . And thirdly, the rings all have $\beta$-parameters which indicate a solely parallel transition. And this excludes the $\mathrm{I}^{+}\left({ }^{1} \mathrm{~S}_{0}\right)+\mathrm{I}\left({ }^{2} \mathrm{P}_{1 / 2}\right)$ asymptotes from the list of possible product combination since from the parallel transition follows that $\Delta J=0$ and thus the fragments must be able to form a state with $\mathrm{J}=3 / 2$.

\subsubsection{Conclusion}

The experimental results and the conclusions from the discussions above are summarized in table 14.11 below. Here the proposed production mechanisms are given as well as the relative intensities of the process belonging to the same excitation scheme. Thus the peaks are grouped by the excitation scheme and the mechanism and then sorted by the TKER. To calculate the ratios within one group the total area of all peaks from this group from one image was set to one and then the relative areas from one image determined. These values were then averaged over all images / measurements. Summarized are the results which have proven to be the most reasonable interpretations of the measured results in the preceding discussion.

\subsection{6 eV feature}

This process which is induced by a VUV and UV photon, with the UV photon determining the polarization dependence, is tried to be matched to either the dissociation of the ionic molecule by $212.55 \mathrm{~nm}$ in table 14.12 and to the three body decay induced with UV+VUV in table 14.13. Because it was checked during the experiments that all iodine ions disappeared when the IR was blocked, which meant that no VUV was produced and only $212.55 \mathrm{~nm}$ light 


\begin{tabular}{|c|c|c|c|c|c|c|}
\hline no. & $\begin{array}{c}\text { TKER } \\
{[\mathrm{eV}]}\end{array}$ & photons & mechanism & $\begin{array}{l}\text { experimental } \\
\text { vert. trans. } \\
\text { energy }[\mathrm{eV}]\end{array}$ & asymptotes & ratio \\
\hline 1 & 0.03 & $2 \mathrm{VUV}$ & diss. ion. $\mathrm{B}^{2} \Sigma_{g}^{+}$ & $12.82 / 12.90$ & ${ }^{2} \mathrm{P}_{3 / 2}+{ }^{3} \mathrm{P}_{0,1}$ & 0.06 \\
\hline 4 & 0.62 & 2 VUV? & diss. ion. $2^{2} \Sigma_{g}^{+}$ & 12.62 & ${ }^{2} \mathrm{P}_{3 / 2}+{ }^{3} \mathrm{P}_{2}$ & 0.07 \\
\hline 5 & 0.78 & $2 \mathrm{VUV}$ & diss. ion. $\mathrm{B}^{2} \Sigma_{g}^{+}$ & 12.77 & ${ }^{2} \mathrm{P}_{3 / 2}+{ }^{3} \mathrm{P}_{2}$ & 0.18 \\
\hline 8 & 3.91 & $2 \mathrm{VUV}$ & diss. ion. $\mathrm{Z}$ & $\begin{array}{c}16.71 / 16.78 \\
16.85\end{array}$ & $\begin{array}{c}{ }^{2} \mathrm{P}_{3 / 2}+{ }^{3} \mathrm{P}_{0,1} \\
{ }^{2} \mathrm{P}_{1 / 2}+{ }^{3} \mathrm{P}_{2}\end{array}$ & 0.28 \\
\hline 9 & 4.88 & $2 \mathrm{VUV}$ & diss. ion. Z & 16.88 & ${ }^{2} \mathrm{P}_{3 / 2}+{ }^{3} \mathrm{P}_{2}$ & 0.29 \\
\hline 10 & 5.77 & $2 \mathrm{VUV}$ & diss. ion. Z' & 19.47 & ${ }^{2} \mathrm{P}_{3 / 2}+{ }^{1} \mathrm{D}_{2}$ & 0.12 \\
\hline no. & $\begin{array}{c}\text { TKER } \\
{[\mathrm{eV}]}\end{array}$ & photons & mechanism & $\begin{array}{c}\text { dissociation } \\
\text { limit }[\mathrm{eV}]\end{array}$ & asymptotes & ratio \\
\hline 3 & 0.41 & $1 \mathrm{VUV}$ & ion pair & 9.79 & $\begin{array}{l}\mathrm{I}^{+}\left({ }^{3} \mathrm{P}_{2}\right) \\
+\mathrm{I}^{-}\left({ }^{1} \mathrm{~S}_{0}\right)\end{array}$ & 0.70 \\
\hline 6 & 1.27 & $1 \mathrm{VUV}$ & ion pair & 8.93 & $\begin{array}{l}\mathrm{I}^{+}\left({ }^{3} \mathrm{P}_{0,1}\right) \\
+\mathrm{I}^{-}\left({ }^{1} \mathrm{~S}_{0}\right) \\
\end{array}$ & 0.30 \\
\hline 2 & 0.14 & $\begin{array}{c}\text { VUV } \\
+ \text { UV? }\end{array}$ & $\begin{array}{c}\text { neutral diss. } \\
\text { by VUV }\end{array}$ & 10.06 & & \\
\hline 7 & 2.16 & $\begin{array}{l}\text { VUV } \\
+ \text { UV }\end{array}$ & 3 body & 13.87 & ${ }^{2} \mathrm{P}_{3 / 2}+{ }^{1} \mathrm{D}_{2}$ & \\
\hline
\end{tabular}

Table 14.11: The experimental results in this work, their corresponding $\beta$ parameters and the production mechanism they are assigned to. The experimental vertical transition energy and the dissociation limits are determined from the TKER and the photon energies. The corresponding asymptotes and the ratios within groups formed by the excitation energies are also given.

was present, dissociation of the $\mathrm{A}^{2} \Pi_{u}$ states by $212.55 \mathrm{~nm}$ is out of question because it would require altogether three UV photons but no VUV. The three body decay suggests itself because of the appearance of the 'peak' as a rather broad distribution which resembles the three body decay processes observed in the UV+ VUV experiments.

If dissociation of the ionic molecule is assumed, the most fitting TKER in table 14.12 is the dissociation of the ionic ground state $\mathrm{X}^{2} \Pi_{3 / 2, g}$ to ${ }^{2} \mathrm{P}_{1 / 2}+{ }^{3} \mathrm{P}_{2}$. But because the distribution around $2.16 \mathrm{eV}$ actually runs from 1.88 to $2.48 \mathrm{eV}$, contributions from all fragmentation asymptotes shown are possible and would actually explain as well why the distribution is so broad. But because so far arguments were in favour for dissociative ionization instead of dissociation of the ionic molecules for all other peaks, it is surprising that now dissociation of the ionic molecule to different channels should be the domi- 


\begin{tabular}{|l||c|c|c|c|}
\hline $\mathrm{I}_{2}^{+} \mathrm{X}^{2} \Pi_{3 / 2, g}$ & & & & \\
\hline \hline $\mathrm{I}^{+}$states & ${ }^{3} \mathrm{P}_{2}$ & ${ }^{3} \mathrm{P}_{0}$ & ${ }^{3} \mathrm{P}_{1}$ & ${ }^{1} \mathrm{D}_{2}$ \\
{$[\mathrm{eV}]$} & 0 & 0.799 & 0.879 & 1.702 \\
\hline I states $[\mathrm{eV}]$ & & & & \\
${ }^{2} \mathrm{P}_{3 / 2} 0$ & & $2.346(2.375)$ & $2.267(2.296)$ & \\
${ }^{2} \mathrm{P}_{1 / 2} 0.943$ & $2.202(2.232)$ & & & \\
\hline \hline $\mathrm{I}_{2}^{+} \mathrm{X}^{2} \Pi_{1 / 2, g}$ & ${ }^{3} \mathrm{P}_{2}$ & ${ }^{3} \mathrm{P}_{0}$ & ${ }^{3} \mathrm{P}_{1}$ & ${ }^{1} \mathrm{D}_{2}$ \\
\hline \hline${ }^{2} \mathrm{P}_{3 / 2} 0$ & & & & $2.088(2.116)$ \\
${ }^{2} \mathrm{P}_{1 / 2} 0.943$ & $2.847(2.875)$ & $2.048(2.076)$ & $1.968(1.997)$ & \\
\hline
\end{tabular}

Table 14.12: Possible combination of dissociation products from the ionic $X\left({ }^{2} \Pi_{g}\right)$ iodine molecule with $212.55 \mathrm{~nm}$ in the experimentally observed TKER range. The first TKER values give the nominal values for $I_{2}^{+}\left(v^{\prime}=0\right)$, the numbers in brackets denote the nominal TKER values for $I_{2}^{+}\left(v^{\prime}=1\right)$.

nant process. This is only conceivable if is assumed that the ionic molecule has a much higher absorption cross section to dissociation for $212.55 \mathrm{~nm}$ than for $121.53 \mathrm{~nm}$ wavelength.

As mentioned, the process here is similar to the wavelength dependent features seen in the VUV+UV experiments. In these experiments it was taken care that this process only appears with both lasers present and all other I atom production from only VUV depending processes was suppressed. It was found (see chapter 15) that this corresponds to three body fragmentation of $\mathrm{I}_{2}+h \nu \longrightarrow \mathrm{I}+\mathrm{I}^{+}+\mathrm{e}^{-}$. Due to the three body fragmentation, the electron can take away every amount of energy. The overall shape is most similar to the low energy distribution seen at $266 \mathrm{~nm}$, but here both ion and neutral atom are produced in an excited state. Possible designations are ${ }^{2} \mathrm{P}_{3 / 2}+{ }^{1} \mathrm{D}_{2}$ and ${ }^{2} \mathrm{P}_{1 / 2}+{ }^{3} \mathrm{P}_{0,1}$, see table 14.13. In the UV+VUV experiments, the peak value was alway $\approx 0.2 \mathrm{eV}$ smaller than the maximum value, thus here the $2.339 \mathrm{eV}$ value of the ${ }^{2} \mathrm{P}_{3 / 2}+{ }^{1} \mathrm{D}_{2}$ matches best. This is actually the same process which was attributed to the dip in the $\beta$-parameter seen in the low energy wavelength dependent feature in the $266 \mathrm{~nm}$ image.

\section{Bibliography}

[1] Ch. Moore. Atomic energy levels. In Ch. Moore, editor, Nat. Stand. Ref. Data: Ser. Nat. Bur. Stand. (US), Vol. 35, chapter 5. 1971.

[2] J. Borkowski. Erzeugung intensiver abstimmbarer gepulster Laserstrahlung im VUV. Diplomarbeit, Universität Bielefeld, Fakultät für Physik, June 1993. 


\begin{tabular}{|l||c|c|c|c|c|}
\hline $\begin{array}{l}\text { 3 body decay } \\
\text { VUV+212.55 nm }\end{array}$ & & & & & \\
\hline \hline $\mathrm{I}^{+}$states & ${ }^{3} \mathrm{P}_{2}$ & ${ }^{3} \mathrm{P}_{0}$ & ${ }^{3} \mathrm{P}_{1}$ & ${ }^{1} \mathrm{D}_{2}$ & ${ }^{1} \mathrm{~S}_{0}$ \\
{$[\mathrm{eV}]$} & 0 & 0.799 & 0.879 & 1.702 & 3.658 \\
\hline $\mathrm{I}^{+}$states $[\mathrm{eV}]$ & & & & & \\
${ }^{2} \mathrm{P}_{3 / 2} 0$ & 4.041 & 3.242 & 3.162 & 2.339 & 0.383 \\
${ }^{2} \mathrm{P}_{1 / 2} 0.943$ & 3.098 & 2.299 & 2.220 & 1.397 & - \\
\hline
\end{tabular}

Table 14.13: Only possible three body decay products from $212.55+121.53 \mathrm{~nm}$ photon.

[3] R.P. Tuckett, E. Castellucci, M. Bonneau, G. Dujardin, and S. Leach. Chem. Physics, 92:43, 1985.

[4] J.H.D. Eland. Jour. Chem. Phys., 70:2926, 1979.

[5] S. Leach. Jour. Phys. Chem., 92:5373, 1988.

[6] D.C. Frost, C.A. Dowell, and D.A. Vroom. Jour. Chem. Phys., 46:4255, 1967.

[7] A.B. Cornford, D.C. Frost, A. McDowell, J.L. Ragle, and I.A. Stenhouse. Jour. Chem. Phys., 54:2651, 1971.

[8] R.G. McLoughlin, J.D. Morrison, and D.L. Smith. Inter. Jour. Mass Spec. and Ion Proc., 58:201, 1984.

[9] W.A. de Jong, L. Visscher, and W.C. Nieuwpoort. Jour. Chem. Phys., 107:9046, 1997.

[10] S. Unny, Y. Du, L. Zhu, R.J. Gordon, A. Sugita, M. Kawasaki, Y. Matsumi, and Tamar Seidelman. Phys. Rev. Lett., 86:2245, 2001.

[11] J.M. Dyke, G.D. Josland, J.G. Snijders, and P.M. Boerrigter. Chem. Physics, 91:419, 1984. 


\section{Chapter 15}

\section{Results at UV+VUV wavelength}

\subsection{Experimental results}

In these measurements the detected $\mathrm{I}^{+}$ions were produced with a combination of VUV photons at $121.53 \mathrm{~nm}$ wavelength and UV photons at either $365 \mathrm{~nm}$ or $266 \mathrm{~nm}$ wavelength. The second laser was introduced into the chamber on an axis which was tilted by 4 degree in respect to the VUV laser. This allows a spatial overlap of the two lasers along roughly $3 \mathrm{~cm}$ in the dissociation volume. It was ensured that the signal depended on both wavelengths, i.e. with both lasers blocked, the signal was near to none.

An example of the raw and converted images for both wavelength combinations are shown in the figure 15.1. The TKER taken from the images are shown in figure 15.2.

The images show altogether five rings from VUV+UV excitation. There are three faint traces at high TKER energies in the $266 \mathrm{~nm}$ image which belong to VUV excitation, compare chapter 14, and are therefore ignored in this section.

Inspection of figure 15.2 reveals that the three peaks at small TKER exhibit the same TKER for both wavelength combinations although the relative intensity and the angular distribution appears to be slightly different for the two combinations (fig. 15.1, right hand side). The two rings with higher TKER display a different behaviour. Their TKER change accordingly to the changed UV wavelength and their distributions are broader, especially the ones at $1.5 \mathrm{eV}(365 \mathrm{~nm})$ and at $1.7 \mathrm{eV}(266 \mathrm{~nm})$.

Thus at least two different fragmentation mechanism are present. 

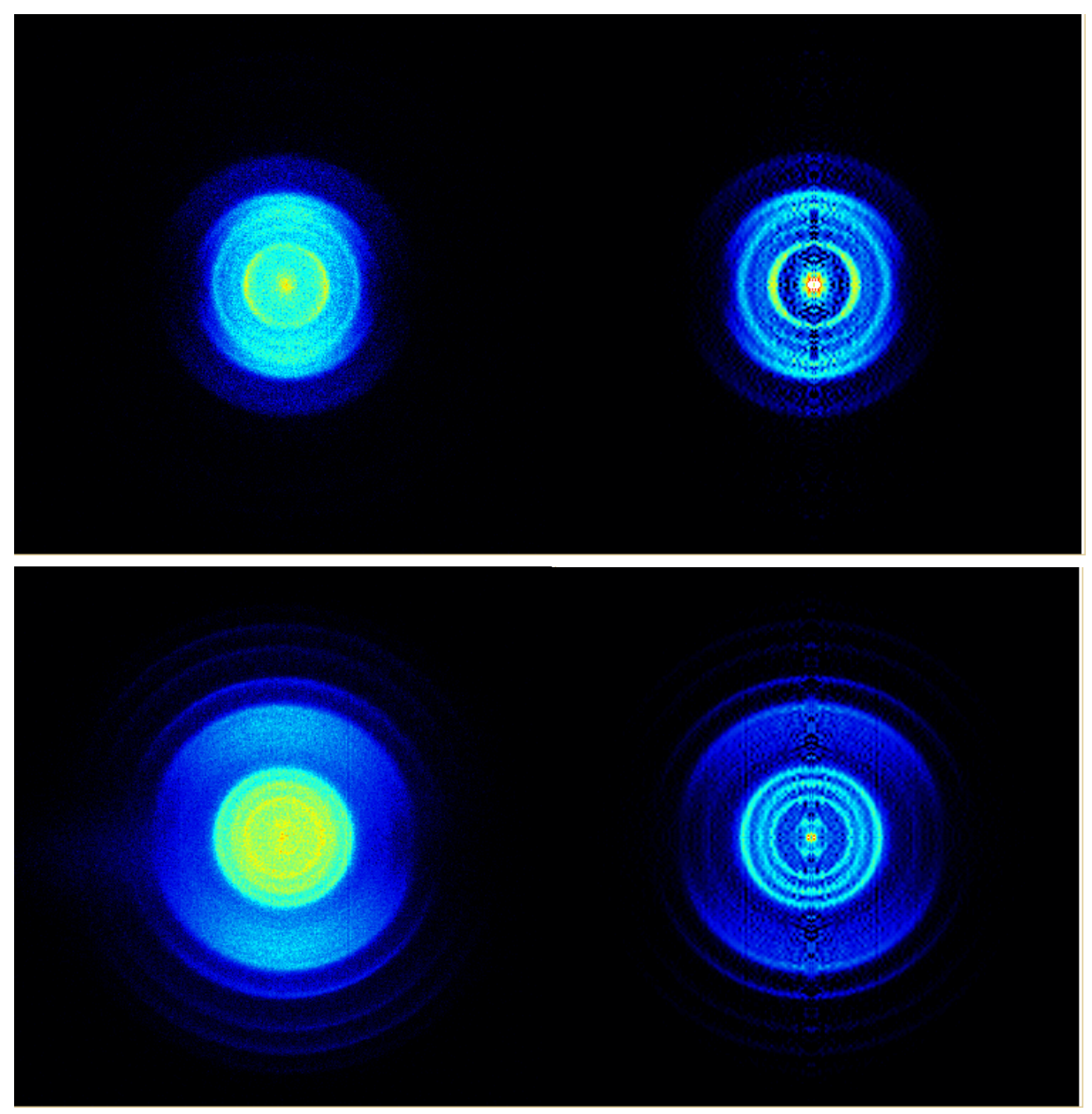

Figure 15.1: Raw and converted images at 121.53+365 $\mathrm{nm}$ wavelength (upper row) and $121.53+266 \mathrm{~nm}$ (lower row). Raw images are on the left, converted images are on the right hand side.

In figure 15.5 the integrated signal of each peak and their corresponding width are shown for measurements on different days. Therefore the experimental conditions are slightly different for each measurement. In the previous chapter about the VUV case (see chapter 14) it was discussed that because of the differences in experimental conditions, the peaks from one measurement exhibited different intensities relative to each other compared to a measurement from a different day, meaning that they depended differently on the 


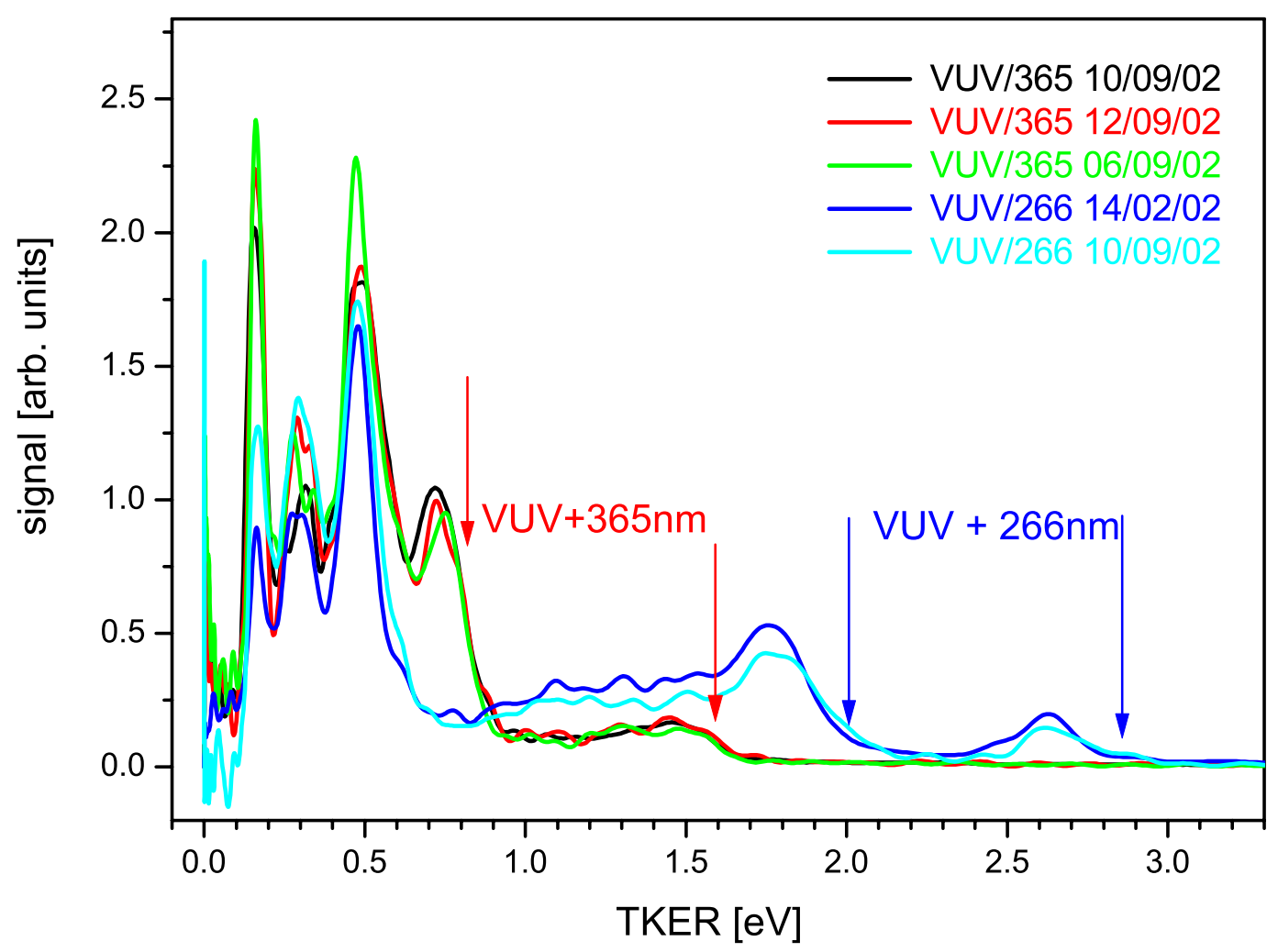

Figure 15.2: The TKER distribution for $V U V+365 \mathrm{~nm}$ and $V U V+266 \mathrm{~nm}$ photons from different days. All distributions are normalized so that the total area equals one. The arrows give the nominal onset of the three body decay for both UV wavelength. The left arrow of each pair indicates the $I^{+}\left({ }^{3} P_{0,1}\right)+I\left({ }^{2} P_{3 / 2}\right)$ channel, whereas the right arrow indicates the $I^{+}\left({ }^{3} P_{2}\right)+I\left({ }^{2} P_{3 / 2}\right)$ channel.

laser power. From these differences it was concluded that some of the processes required absorption of either one VUV photon, two VUV-photons or a combination of one VUV photon plus one UV photon from the $212.55 \mathrm{~nm}$ residue present from the VUV production.

This kind of behaviour was not observed here. For different experimental conditions the relative intensities of the peaks always stayed the same, thus always the same combination of photons was absorbed for both different fragmentation mechanism. Because I ensured during the measurements that the signal depended on both lasers which required rather low laser energies, the assumption is reasonable that one VUV and one UV photon was absorbed. The photon energies are then $13.60 \mathrm{eV}(\mathrm{VUV}+365 \mathrm{~nm})$ and $14.86 \mathrm{eV}$ $(\mathrm{VUV}+266 \mathrm{~nm})$. 


\begin{tabular}{|c|c||c|c|c|c|}
\hline exp. value $[\mathrm{eV}]$ & $\begin{array}{c}\text { range } \\
{[\mathrm{eV}]}\end{array}$ & $\beta$ & $\begin{array}{c}\text { range } \\
\beta\end{array}$ & area & width $[\mathrm{eV}]$ \\
\hline $266 \mathrm{~nm}$ & & & & & \\
\hline \hline 0.16 & \pm 0.001 & 0.07 & 0.17 & 0.08 & 0.07 \\
\hline 0.29 & \pm 0.005 & 0.32 & 0.17 & 0.17 & 0.17 \\
\hline 0.48 & \pm 0.01 & -0.17 & 0.01 & 0.28 & 0.14 \\
\hline $\begin{array}{c}1.19 \\
\text { other channel ? } \\
\text { see text }\end{array}$ & \pm 0.02 & 0.87 & 0.02 & - & - \\
\hline $\begin{array}{c}1.74 \\
\text { onset of feature } \\
\text { at 2.01 }\end{array}$ & \pm 0.03 & 1.59 & 0.001 & 0.41 & 0.92 \\
\hline $\begin{array}{c}2.60 \\
\text { onset of feature } \\
\text { at 2.84 }\end{array}$ & \pm 0.02 & 1.85 & 0.07 & 0.05 & 0.26 \\
\hline \hline \begin{tabular}{c}
$365 \mathrm{~nm}$ \\
\hline \hline 0.16
\end{tabular} & \pm 0.002 & -0.43 & 0.25 & 0.16 & 0.06 \\
\hline 0.31 & \pm 0.004 & 0.21 & 0.18 & 0.15 & 0.14 \\
\hline 0.48 & \pm 0.01 & -0.03 & 0.13 & 0.37 & 0.16 \\
\hline $\begin{array}{c}0.74 \\
\text { onset feature } \\
\text { at } 0.86\end{array}$ & \pm 0.02 & 1.72 & 0.13 & 0.22 & 0.20 \\
\hline $\begin{array}{c}1.44 \\
\text { onset of feature } \\
\text { at } 1.70\end{array}$ & \pm 0.01 & & & & \\
\hline
\end{tabular}

Table 15.1: The experimental results in this work at VUV/UV photons, their corresponding beta parameters, the integrated signal of the peaks and their width. The integrated signal is normalized for each image. Range [eV] means the maximum value by which the peak position deviated from the average value all used images considered. Range $\beta$ describes the difference between the maximum and minimum value determined in different measurements for one peak, see text.

The experimental results are summarized in table 15.1.

Here, the energy ranges of the peaks are similar to the ranges found in the VUV case, although here only two $(266 \mathrm{~nm})$ respectively three $(365 \mathrm{~nm})$ images were evaluated, whereas in the VUV case nine images contributed to the final results. Normally one would expect that for a small number of images, the range would be smaller and falsely indicating a smaller error and 
higher precision. But since the values are comparable, it can be concluded that they represent the real error quite well. On the other hand, the $\beta$ parameter here has a much smaller range compared to the VUV case, thus the real error is realistically higher than the range given here and more of the order observed in the VUV case $(\approx 0.5)$.

\subsection{UV wavelength independent rings}

The rings which do not depend on the wavelength of the UV photon must adhere to a mechanism which needs the UV photon, but where the excess energy of that photon is carried away by the electron, since the TKER does not change. Possible production mechanism are discussed in the following sections.

\subsubsection{Excitation to a repulsive state}

One possible mechanism how the wavelength independent rings are produced is direct ionization to a repulsive state, analogous to the excitation found in the VUV images.

A schematic picture of fragmentation via a repulsive state is given in fig. 15.3. The $\mathrm{B}^{2} \Sigma_{g}^{+}$state is shown as the assumed double potential curve [1] dissociating to either $\mathrm{I}^{+}\left({ }^{3} \mathrm{P}_{2}\right)+\mathrm{I}\left({ }^{2} \mathrm{P}_{3 / 2}\right)$ or $\mathrm{I}^{+}\left({ }^{3} \mathrm{P}_{0,1}\right)+\mathrm{I}\left({ }^{2} \mathrm{P}_{3 / 2}\right)$ asymptotes by coupling onto the lower curve.

The repulsive state is reached in direct ionization and for example dissociates directly to ground state products. The TKER is then given by the energy gap between the vertical excitation energy of the repulsive state and the appropriate asymptotes. Or vice versa, the vertical excitation energy is given by the measured TKER plus the minimum energy required to reach this asymptotes, given by the dashed vertical lines in grey.

The TKER release found for the first three rings is $0.16,0.30$ and $0.48 \mathrm{eV}$. The excitation energy is either 13.60 or $14.86 \mathrm{eV}$. For the former, the photon energy is sufficient for the $\mathrm{I}^{+}\left({ }^{3} \mathrm{P}_{2,0,1}\right)+\mathrm{I}\left({ }^{2} \mathrm{P}_{3 / 2}\right)$ and $\mathrm{I}^{+}\left({ }^{3} \mathrm{P}_{2}\right)+\mathrm{I}\left({ }^{2} \mathrm{P}_{1 / 2}\right)$ product channels. The minimum energy required to produce $\mathrm{I}^{+}\left({ }^{1} \mathrm{D}_{2}\right)+\mathrm{I}\left({ }^{2} \mathrm{P}_{3 / 2}\right)$ is $0.096 \mathrm{eV}$ above the photon energy value of $13.60 \mathrm{eV}$ obtained with $\mathrm{VUV}+365 \mathrm{~nm}$. Thus, the threshold for the production of $\mathrm{I}^{+}\left({ }^{1} \mathrm{D}_{2}\right)+\mathrm{I}\left({ }^{2} \mathrm{P}_{3 / 2}\right)$ can be reached only from vibrational level with $\mathrm{v}>3$ of the molecular ground state $\mathrm{X}^{1} \Sigma\left(0_{g}^{+}\right)$. Additionally, for the vertical ionization energy to a corresponding repulsive state the measured TKER must be added, making it at least nine vibrational quanta and thus this channel is excluded for the longer UV wavelength. For the latter excitation energy, all combination 


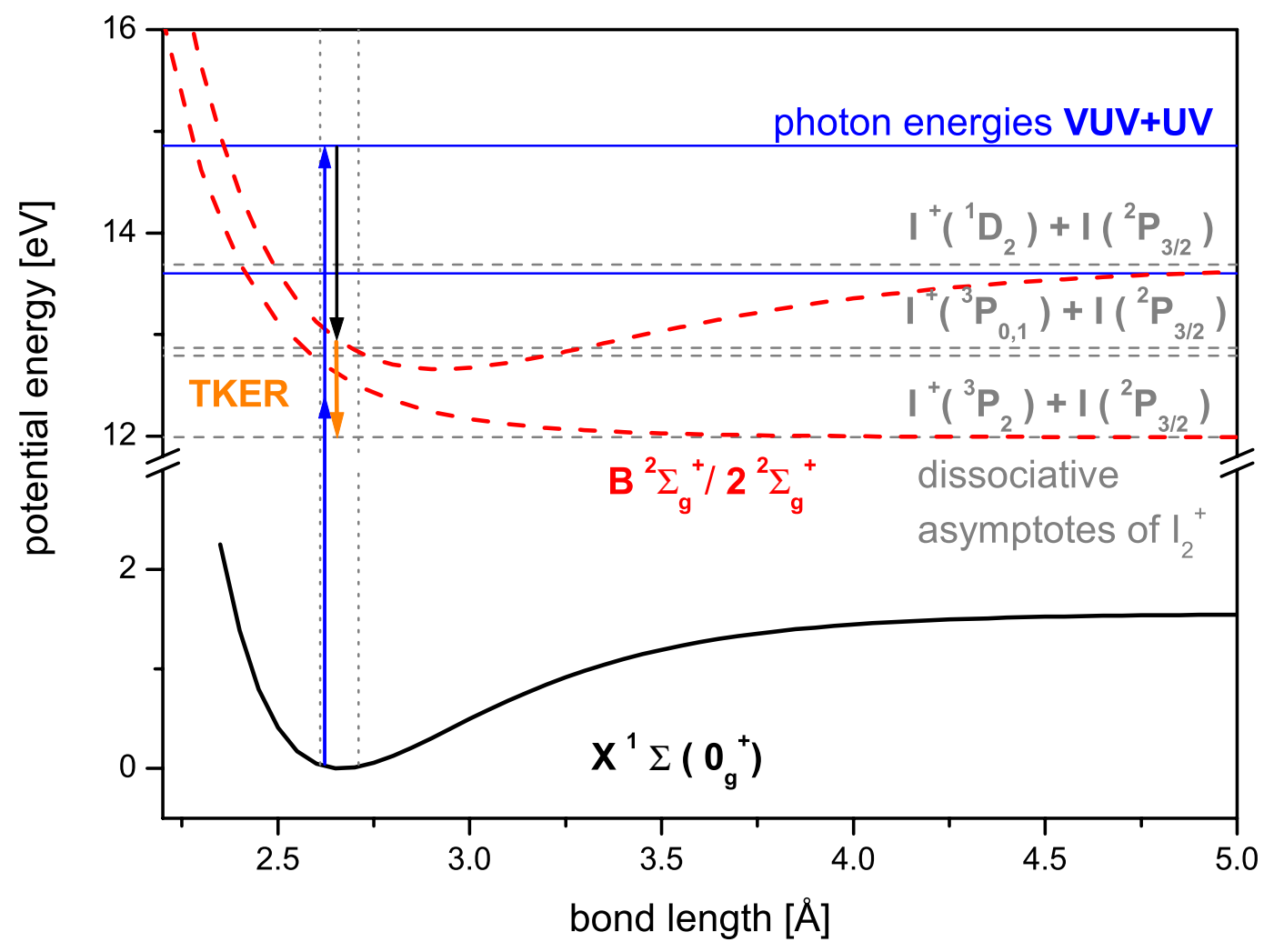

Figure 15.3: Schematic potential curves illustrating the mechanism of direct ionizing to a repulsive curve with $V U V+U V$ photons. Dashed lines correspond to ionic states, solid ones to the neutral. Grey dashed vertical lines indicate the ionic asymptotes, the grey dotted lines the Frank-Condon window from the ground state. The two blue lines show the two excitation energies achieved with one VUV plus one UV photon. The black arrow represents the electron, the blue arrows the photons and the orange arrow the TKER.

from $\mathrm{I}^{+}\left({ }^{3} \mathrm{P}_{2,0,1}\right)+\mathrm{I}\left({ }^{2} \mathrm{P}_{3 / 2,1 / 2}\right)$ as well as $\mathrm{I}^{+}\left({ }^{1} \mathrm{D}_{2}\right)+\mathrm{I}\left({ }^{2} \mathrm{P}_{3 / 2}\right)$ are accessible, but since for both UV wavelengths the rings are nearly identical, all channels excluded for the VUV+365 nm excitation can quite probably be excluded for VUV $+266 \mathrm{~nm}$ excitation too. Hence only the channels $\mathrm{I}^{+}\left({ }^{3} \mathrm{P}_{2,0,1}\right)+\mathrm{I}\left({ }^{2} \mathrm{P}_{3 / 2}\right)$ and $\mathrm{I}^{+}\left({ }^{3} \mathrm{P}_{2}\right)+\mathrm{I}\left({ }^{2} \mathrm{P}_{1 / 2}\right)$ need to be considered.

If ground state products are assumed, the vertical excitation energy obtained as above described would be 12.16, $12.29,12.47 \mathrm{eV}$. None of these matches known states. If spin orbit excitation is involved, the values would be correspondingly higher. This gives for the $0.16 \mathrm{eV}$ peak and $\mathrm{I}^{+}\left({ }^{3} \mathrm{P}_{0}\right)+\mathrm{I}\left({ }^{2} \mathrm{P}_{3 / 2}\right)$ products a vertical excitation energy of $12.96 \mathrm{eV}$, which matches the value 
for $\mathrm{B}^{2} \Sigma_{g}^{+}$of $12.95 \mathrm{eV}$ given by Tuckett et al. [2] quite well. The other rings unfortunately give values of 13.09 and $13.27 \mathrm{eV}$, which are clearly above the the range usually given for the $\mathrm{B}^{2} \Sigma_{g}^{+}$state. All other energetically possible combinations do give even higher values, to which the same applies.

And there are arguments against the designation of the first peak at $0.16 \mathrm{eV}$ as belonging to $\mathrm{B}^{2} \Sigma_{g}^{+}$. The first is a plausibility argument. It is simply not reasonable that only one value is assigned to dissociation via a repulsive state if all other experimental TKER values do not match a known repulsive state. The second argument is that the TKER value which was assigned to the $\mathrm{B}^{2} \Sigma_{g}^{+}$state in the VUV image is at $0.03 \mathrm{eV}$. Of course one can argue that this assignment is not correct, but firstly the excitation energies used in the VUV images resemble more the conditions of other experiments the experimental result of which were partly reproduced in the VUV experiments and secondly the channel to ground state products is missing which is known from other experiments to be existent [2].

Unny et al. [3] point out that if a resonant intermediate is met with one photon, the Frank-Condon window may shift and the vertical transition energy is slightly altered. But if this is the case here, firstly, this shift will be a fixed value and not apt to explain three different energies, and secondly, the resonant step is more likely to be made with VUV since the UV wavelength is changed, and if the VUV meets a high lying Rydberg state and the subsequent step is from there, there is no reason why this shift in energy should only be observed here and not in the experiments with two VUV photons.

Therefore it can be concluded that excitation to a repulsive state is not the case here.

\subsubsection{Dissociation into two neutral I atoms}

Another process which would result in UV wavelength independent behaviour is dissociation to neutral fragments with one VUV photon, where one fragment is excited to a high Rydberg state which can subsequently be ionized by the UV photon.

Possible designations of the products are given in table 15.2 below. Mostly, a variety of possible states is given which match the experimental energy release within experimental error. Do determine experimentally which assignment is correct, resonant ionization or fluorescence experiments would have been appropriate, but were not pursued due to lack of time.

However, some thought can be given to possibly involved molecular Rydberg states. Since the energy of one VUV photon is big enough to reach the ionization limit to $\mathrm{I}_{2}^{+}\left(\mathrm{X}^{2} \Pi_{3 / 2, g}\right)$ and $\mathrm{I}_{2}^{+}\left(\mathrm{X}^{2} \Pi_{1 / 2, g}\right)$ the Rydberg states based on these cores can be safely excluded. Unfortunately, little is known about 


\begin{tabular}{|l||c|c|c|}
\hline TKER & 0.16 & 0.30 & 0.48 \\
\hline \hline term value of I* $[\mathrm{eV}]$ & 8.50 & 8.36 & 8.18 \\
with I* $+{ }^{2} \mathrm{I}_{3 / 2}$ & & & \\
\hline $\begin{array}{l}\text { possible designations } \\
\text { of I* }\end{array}$ & $\left({ }^{1} D_{2}\right) 6 \mathrm{~s}[2]_{5 / 2}$ & $\left({ }^{3} P_{2}\right) 5 \mathrm{~d}[4]_{9 / 2}$ & $\left({ }^{3} P_{2}\right) 6 \mathrm{p}[1]_{1 / 2}$ \\
and its & 8.504 & 8.397 & 8.165 \\
literature & $\left({ }^{1} D_{2}\right) 6 \mathrm{~s}[2]_{3 / 2}$ & $\left({ }^{3} P_{2}\right) 6 \mathrm{p}[1]_{3 / 2}$ & \\
term values $[\mathrm{eV}]$ & 8.499 & 8.315 & \\
& $\left({ }^{3} P_{2}\right) 5 \mathrm{~d}[4]_{7 / 2}$ & & \\
& 8.500 & & \\
& $\left({ }^{3} P_{2}\right) 5 \mathrm{~d}[0]_{1 / 2}$ & & \\
\hline \hline term value $[\mathrm{eV}]$ & 8.507 & & 7.23 \\
with I $+{ }^{2} \mathrm{I}_{1 / 2}$ & 7.55 & 7.42 & \\
\hline possible designations & $\left({ }^{3} P_{0}\right) 6 \mathrm{~s}[0]_{1 / 2}$ & & \\
of I* and its & 7.550 & & \\
literature term values $[\mathrm{eV}]$ & & & \\
\hline
\end{tabular}

Table 15.2: Possible designation of $I^{*}$ in the dissociation of $I_{2}$ by one VUV photon at $121.6 \mathrm{~nm}$. The literature values are taken from ref. [4].

the Rydberg states converging onto the $\mathrm{A}^{2} \Pi_{u}$ or $\mathrm{B}^{2} \Sigma_{g}^{+}$states. Some data is available from the absorption spectra by Venkateswarlu [5], whose assignments were slightly revised by later works [6], but at large still constitute the accepted standard. But his data only cover transition energies up to the first ionization limit and the states he assigned to $\left[\mathrm{A}^{2} \Pi_{3 / 2, u}\right]_{c}$ or $\left[\mathrm{A}^{2} \Pi_{1 / 2, u}\right]_{c}$ Rydberg states have maximum vertical transition energies of $9.10 \mathrm{eV}$. Kvaran et al. discuss in their paper concerning free ion pair states [7] that the opening of the ion pair channel $\mathrm{I}^{+}\left({ }^{3} \mathrm{P}_{0,1}\right)+\mathrm{I}^{-}\left({ }^{1} \mathrm{~S}_{0}\right)$ at $126 \mathrm{~nm}$ or $9.84 \mathrm{eV}$ coincides with the $\left[\mathrm{A}^{2} \Pi_{3 / 2, u}\right]_{c} 8 \mathrm{~s}$ Rydberg system. Cockett et al. [8] give the approximate regions of some Rydberg states converging onto electronically excited $\mathrm{I}_{2}^{+}$states, Ridley et al. [6] re-analysed the spectrum of Venkateswarlu and augmented it with their own data. From these different works, the Rydberg states converging onto the $\mathrm{A}^{2} \Pi_{u}$ and $\mathrm{B}^{2} \Sigma_{g}^{+}$states are summarized in table 15.3 .

Because the $\mathrm{A}^{2} \Pi_{u}$ states have a longer bondlength $(3 \AA)$ than the $\mathrm{X}^{2} \Pi_{g}$ states $(2.6 \AA)$, a vertical transition from the ground state $\mathrm{X}^{1} \Sigma\left(0_{g}^{+}\right)$to a $\left[\mathrm{A}^{2} \Pi_{u}\right]_{c}$ Rydberg state will go to the repulsive part of the $\left[\mathrm{A}^{2} \Pi_{u}\right]_{c}$ Rydberg state. Thus either high vibrational quanta result or the absorption into the dissociation continuum is facilitated.

Since the highly excited fragment of two of the measured channels $(0.16$ 


\begin{tabular}{|c|c|c|c|}
\hline core & $\begin{array}{c}\text { Rydberg } \\
\text { series }\end{array}$ & $\begin{array}{c}\text { range } \\
{[\mathrm{eV}]}\end{array}$ & $\begin{array}{c}\text { taken } \\
\text { from }\end{array}$ \\
\hline \hline$\left[\mathrm{A}^{2} \Pi_{3 / 2, u}\right]_{c}$ & $7 \mathrm{p}$ & 9.68 to 9.92 & {$[8]$} \\
& $8 \mathrm{p}$ & 10.17 to 10.51 & {$[8]$} \\
& $5 \mathrm{~d}$ & $8.54-8.62$ & {$[6]$} \\
& $5 \mathrm{~d}$ & $>9.17$ & {$[6]$} \\
& $6 \mathrm{~s} \sigma_{g} \Pi_{1, u}$ & 7.88 & {$[5]$} \\
& $5 \mathrm{~d} \sigma_{g} \Pi_{1, u}$ & 8.26 & {$[5]$} \\
& $5 \mathrm{~d} \pi_{g} \Sigma^{+}\left(0_{u}^{+}\right)$ & 8.38 & {$[5]$} \\
& $4 \mathrm{f} \delta_{u} \Pi_{1, u}$ & 8.03 & {$[5]$} \\
& $8 \mathrm{~s}$ & 9.84 & {$[7]$} \\
\hline$\left[\mathrm{A}^{2} \Pi_{1 / 2, u}\right]_{c}$ & $7 \mathrm{p}$ & 10.17 to 10.51 & {$[8]$} \\
& $5 \mathrm{~d}$ & $>9.17$ & {$[6]$} \\
& $6 \mathrm{~s}$ & 7.56 to 8.31 & {$[6]$} \\
& $5 \mathrm{~d} \sigma_{g} \Pi_{1, u}$ & 9.00 & {$[5]$} \\
& $5 \mathrm{~d} \sigma_{g} \Pi\left(0_{u}^{+}\right)$ & 9.01 & {$[5]$} \\
& $5 \mathrm{~d} \pi_{g} \Sigma^{+}\left(1_{u}\right)$ & 9.10 & {$[5]$} \\
& $6 \mathrm{~s} \sigma_{g} \Pi\left(0_{u}^{+}\right)$ & 8.80 & {$[5]$} \\
& $6 \mathrm{~s} \sigma_{g} \Pi_{1, u}$ & 8.78 & {$[5]$} \\
\hline$\left[\mathrm{B}^{2} \Sigma_{g}^{+}\right]_{c}$ & $6 \mathrm{~s}$ & $9.30-10.04$ & {$[8]$} \\
& $5 \mathrm{~d}, 7 \mathrm{~s}$ & $\approx 10.72$ & {$[8]$} \\
\hline
\end{tabular}

Table 15.3: Rydberg states which converge onto electronically excited $\mathrm{I}_{2}^{+}$ states $[8,6,5]$

and $0.30 \mathrm{eV}$ TKER, table 15.2$)$ most likely belongs to the $\left({ }^{3} \mathrm{P}_{2}\right) 5 \mathrm{~d}$ atomic Rydberg series, the molecular Rydberg states should as well be 5d Rydberg states based on an ionic state dissociating to ${ }^{3} \mathrm{P}_{2}+{ }^{2} \mathrm{P}_{3 / 2}$, which is the case for both $\mathrm{A}^{2} \Pi_{u}$ states. Ridley et al. [6] give lower limits for $\left[\mathrm{A}^{2} \Pi_{3 / 2, u}\right]_{c} 5 \mathrm{~d}$ and $\left[\mathrm{A}^{2} \Pi_{1 / 2, u}\right]_{c} 5 \mathrm{~d}$ Rydberg states at $9.17 \mathrm{eV}$, where the authors found yet unassigned bands. If it is assumed that the Rydberg states have the same form and dissociation energy as the appropriate ionic states, values for the asymptotes result around $10.05 \mathrm{eV}$ and $9.35 \mathrm{eV}$ respectively, see fig. 15.4, where these two states are illustrated in context with the ionization limits.

The first value matches quite well the $0.16 \mathrm{eV}$ TKER release value, which would give experimentally a value of $10.04 \mathrm{eV}$ for the asymptote. Although this is only a lower limit of the Rydberg state, it gives support to the assumed mechanism.

The second value is far below the measured value of $0.30 \mathrm{eV}$, which gives experimentally an asymptote of $9.90 \mathrm{eV}$, thus the real Rydberg state involved 


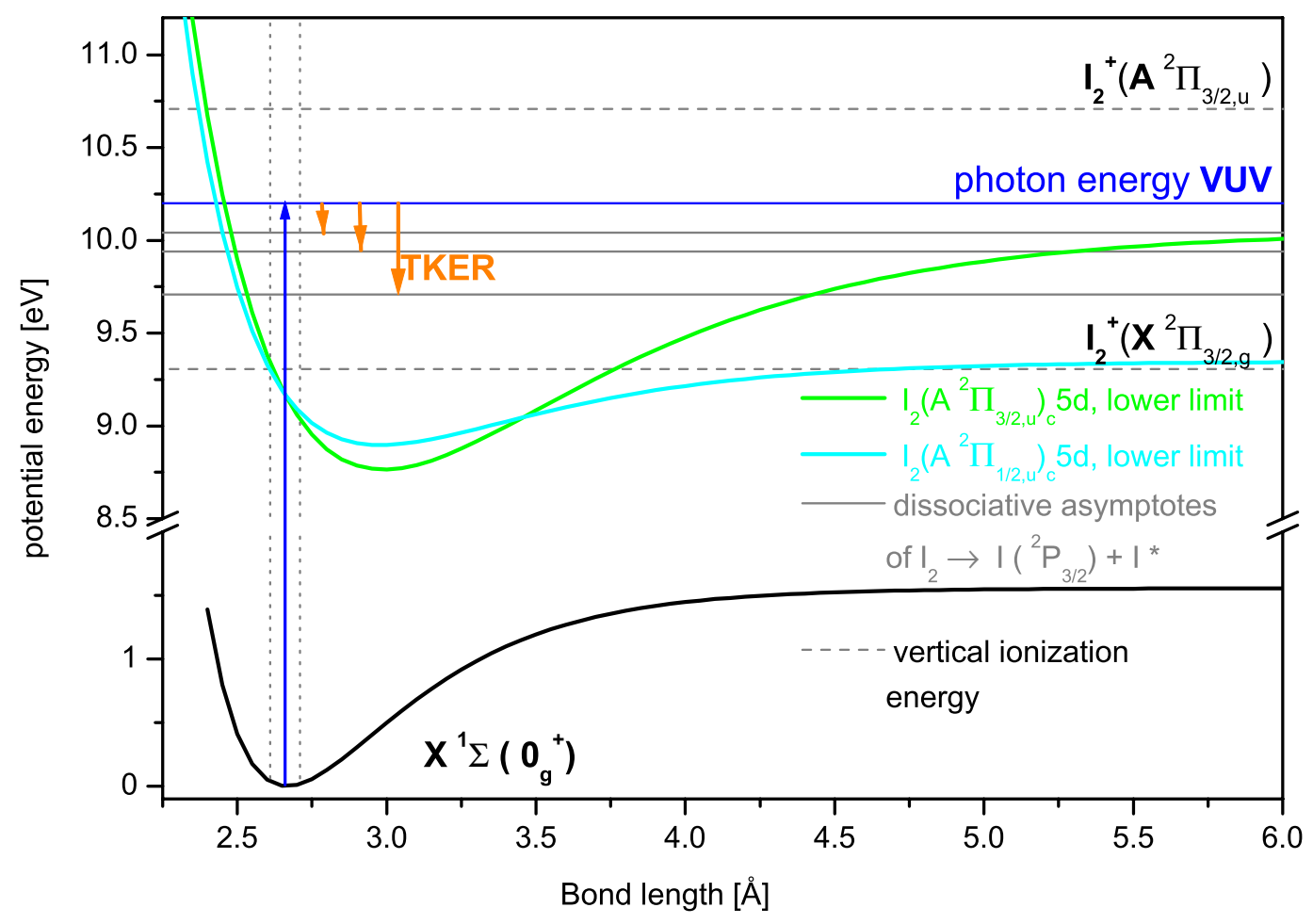

Figure 15.4: Schematic potential curves illustrating dissociation to neutral fragments. Dashed lines correspond to ionic states, solid ones to the neutral. The grey solid line shows the neutral dissociative asymptotes to appropriate products as given in table 15.2, grey dashed horizontal lines indicate the ionization limits, the grey dotted lines the Frank-Condon window from the ground state. The blue line show the excitation energy achieved with one VUV photon. Blue arrows represent photons, the orange arrow represents the TKER. The shown lower limits of the Rydberg states were constructed as follows: Firstly, the $A^{2} \Pi_{u}$ potential curves were generated from the vertical transition energies, the known dissociation energy and the asymptotic value, using a simple Morse potential, since the exact form is not known. Then, these curves are translated to the appropriate vertical transition energy stated as lower limit by Ridley at al. [6].

here can be either a higher $\left[\mathrm{A}^{2} \Pi_{1 / 2, u}\right]_{c}$ state or one based on $\left[\mathrm{A}^{2} \Pi_{3 / 2, u}\right]_{c}$ as well. The third measured value of $0.48 \mathrm{eV}$, giving a value for the asymptote of $9.72 \mathrm{eV}$, is assigned to fragmentation to an atomic $\left({ }^{3} \mathrm{P}_{2}\right) 6 \mathrm{p}$ Rydberg state, which is a possible assignment for the $0.30 \mathrm{eV}$ TKER value too, see table 15.2. Unfortunately, there are no experimental data for either $\left[\mathrm{A}^{2} \Pi_{3 / 2, u}\right]_{c} 6 \mathrm{p}$ and $\left[\mathrm{A}^{2} \Pi_{1 / 2, u}\right]_{c} 6 \mathrm{p}$ Rydberg states in the literature, thus any exact assignment 
is more or less a random choice.

Still there are a lot of unassigned bands in this congested region, thus making in general a transition into the dissociation continuum of a Rydberg state feasible. Finally, the possibility that a Rydberg state based on the $\mathrm{B}^{2} \Sigma_{g}^{+}$state is involved should be considered. Cockett et al. [8] give approximate values for some $\mathrm{B}^{2} \Sigma_{g}^{+}$states, where the value of the $7 \mathrm{~s}$ and $5 \mathrm{~d}$ state is within the asymptotes I obtained in my measurements. But it is not entirely clarified which asymptotes belong to it. There is evidence that its asymptotes are rather ${ }^{3} \mathrm{P}_{0,1}+{ }^{2} \mathrm{P}_{3 / 2}$ or ${ }^{1} \mathrm{D}_{2}+{ }^{2} \mathrm{P}_{3 / 2}$ [9] and that the observed experimental ground state fragmentation products [10] are achieved by extensive configuration mixing. Thus it is questionable, if any conclusion at all can be made from the ionic $\mathrm{B}^{2} \Sigma_{g}^{+}$state concerning the behaviour of the Rydberg states converging onto it.

The dissociation into one ground state fragment and one Rydberg state iodine atom was already proposed by Mulliken [11], but so far only the dissociation or predissociation to $\mathrm{I}\left(6 \mathrm{~s}^{4} \mathrm{P}_{5 / 2}\right)$ and $\mathrm{I}\left(6 \mathrm{~s}^{2} \mathrm{P}_{3 / 2}\right)$ was observed experimentally via vacuum ultraviolet fluorescence of the Rydberg fragments by Hiraya et al. [12].

After discussing the two possible mechanisms, ionization to a repulsive $\mathrm{I}_{2}^{+}$state and dissociation via a Rydberg state into neutral atoms, I come to the conclusion that the dissociation to two neutrals is indeed the case here.

At last, I would like to comment on one feature of the experimental images. Interestingly, the rings seem to have slightly different $\beta$-parameter (fig. 15.6) when probed with 365 or $266 \mathrm{~nm}$, as well as a different relative intensity (fig. 15.5). Since the dissociation step is obviously equal to both measurements and thus certainly initiated by the VUV photon, it points to the ionization step with UV photon as the root of the problem. If the ionization step has different probabilities with different wavelengths, i.e. the absorption coefficients depend strongly on the wavelength, the different relative intensities are easily explained. This may be the case since the ionization energies exceed the needed energy by roughly $1 \mathrm{eV}(365 \mathrm{~nm})$ and $2.3 \mathrm{eV}(266 \mathrm{~nm})$, which may cause a difference in ionization efficiency.

The different $\beta$-parameters are not that easily explained. Alignment effects may play a role, but due to lack of further evidence this remains pure speculation.

On the other hand, limitations of the experiment should be taken into account.

If it is assumed that the distribution at $0.74 \mathrm{eV}$ in the $365 \mathrm{~nm}$ image is similarly broad as the corresponding distribution in the $266 \mathrm{~nm}$ image, it 


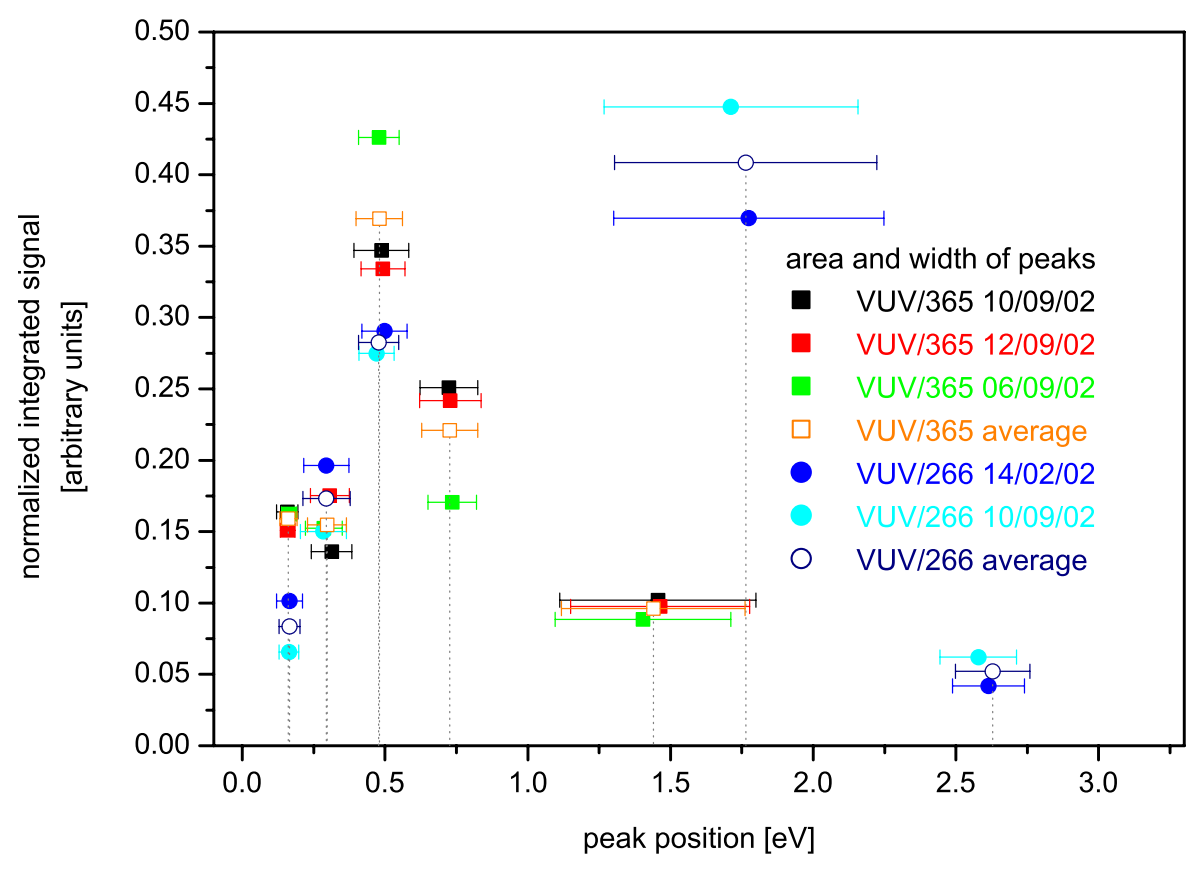

Figure 15.5: The integrated signal of the peaks and their corresponding width. The hollow symbols represent the average values at the averaged peak position as given in table 15.1. The integrated signal was normalized to one.

superimposes on the feature at $0.48 \mathrm{eV}$. Since the correct form of the peak at $0.74 \mathrm{eV}$ is not known, it is impossible to obtain the integrated signal correctly. Thus I just assumed that the amount superimposed by one peak on the other is equal to the amount vice versa and divided the peaks at the minimum in between them. Any integration of signal was then done between this point and the appropriate limit on the other side of the peaks. This is of course not exact but can be justified. Since the amount of superimposed signal is already clearly smaller than the integrated signal of the peak itself, any difference between those amounts of superimposed signal is definitely much smaller compared to the whole signal of the peaks and can be neglected within experimental error.

Now it will be considered if this can also influence the $\beta$-parameters. In the $365 \mathrm{~nm}$ image they are a bit lower for the two rings with the smallest TKER than in the $266 \mathrm{~nm}$ image, but a bit higher for the ring at $0.48 \mathrm{eV}$ neighbouring onto the ring at $0.74 \mathrm{eV}$. It may be that here the $\beta$-parameter is as well a superposition of the real $\beta$-parameter of the ring at $0.48 \mathrm{eV}$ and of the superimposed fraction of the feature peaking at $0.74 \mathrm{eV}$ which has a highly parallel angular distribution. This is supported by the observations for the 


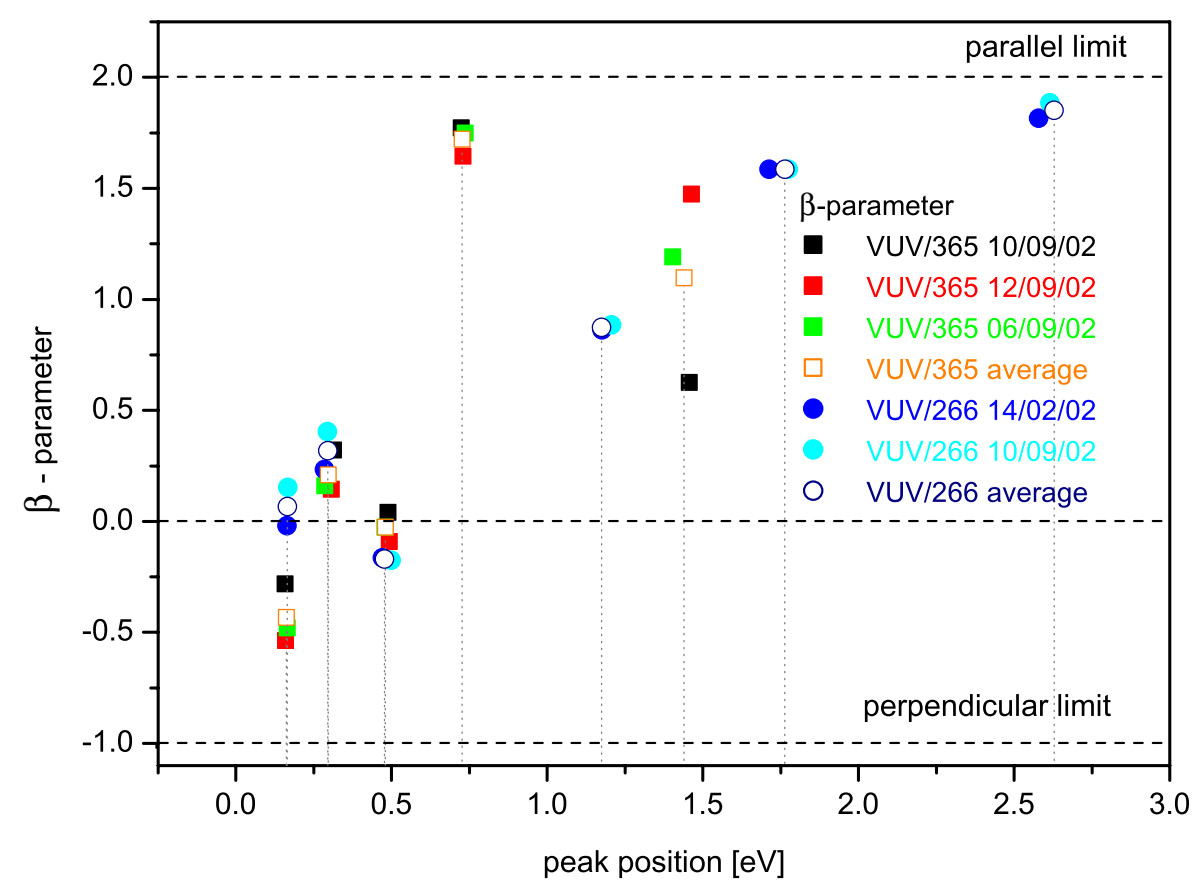

Figure 15.6: $\beta$-parameters of the UV/VUV experiments. The hollow symbols represent the averaged values at the averaged peak position as given in table 15.1.

$121.53 \mathrm{~nm}$ only dissociation processes which are reported in section 14.2 . It was observed that the $\beta$-parameters determined from different measurement covered quite a range of values, thus I believe that here a similar range can be assumed.

It seems here that the $\beta$-parameters variate less, see fig. 15.6, at least all data entries for the UV wavelength $365 \mathrm{~nm}$ are either lower or higher than the entries for the $266 \mathrm{~nm}$ wavelength, which may show that the general trend of differences in the $\beta$-parameter for the two different UV wavelengths are real, but it should be kept in mind that less images were evaluated than in the VUV case.

If a similar behaviour is assumed as in the VUV case, it is surprising that the $\beta$-parameter for $365 \mathrm{~nm} \mathrm{UV}$ wavelength is lower for the 0.16 and $0.29 \mathrm{eV}$ peaks, but higher for the $0.48 \mathrm{eV}$ peak than for $266 \mathrm{~nm}$ UV wavelength. The $\beta$-parameters determined in the VUV case (see fig. 14.3) usually showed the same behaviour for all peaks, meaning that they were either lower or higher for all peaks than the values obtained in different measurements. This supports the idea that the peak at $0.48 \mathrm{eV}$ is actually a superposition of the 'real' peak and the broad distribution peaking at $0.74 \mathrm{eV}$ and that the 'real' $\beta$ - 
parameter is actually in line with the others at $365 \mathrm{~nm}$, which means slightly smaller than the one at $266 \mathrm{~nm}$. Although then it must be also assumed that the differences between the values for $365 \mathrm{~nm}$ and $266 \mathrm{~nm}$ are just within the range and that the fact that the ones for $365 \mathrm{~nm}$ are all in the low value part of the range and the ones for $266 \mathrm{~nm}$ in the high value part of the range is just coincidence.

However, even if this is all rather speculative, the conclusion from these considerations is that the difference in the $\beta$-parameters is probably not real, which supports the picture of the dissociation to neutral fragments by VUV and subsequent ionization by two different UV-wavelengths. If the ionization to a dissociative ionic state with both photons holds true, different ionization efficiencies may cause different relative intensities for the two image, but since the $\beta$-parameter mainly depends on the symmetry of the two states in between the transition takes place (ignoring secondary effects like loss of angular information due to long lifetimes and high rotational excitation), the images should exhibit equal $\beta$-parameters.

\subsection{UV wavelength dependent rings}

As mentioned in the introductory words of this chapter, the two outer rings of each picture change in diameter, i.e. speed, with changing the UV wavelength, with the rings at $1.5 \mathrm{eV}(365 \mathrm{~nm})$ and $1.7 \mathrm{eV}(266 \mathrm{~nm})$ exhibiting a broader distribution than usual. This behaviour is actually similar to the $212.55 \mathrm{~nm}+\mathrm{VUV}$ ring seen in the VUV experiment.

Now, a mechanism has to be found that can provoke this kind of behaviour, especially the broad features.

There are some possibilities which should be considered. Firstly, dissociation from a ionic bound state; secondly, an involvement of intermediate states below the the dissociative step and thirdly, a process where an intermediate state above the final dissociative state is involved.

\subsubsection{Dissociation of an ionic bound state}

If the intermediate in question is actually a bound molecular ionic state which could be the $\mathrm{X}^{2} \Pi_{3 / 2, g}$ and $\mathrm{X}^{2} \Pi_{1 / 2, g}$ states reached with one VUV photon, energy is lost in the ionizing step and the subsequent dissociation step would be governed by $E_{\text {TKER }}=E_{h \nu}(U V)+E_{\text {int }}\left(\mathrm{I}_{2}^{+}\right)-E_{\text {int }}(I)-E_{\text {int }}\left(\mathrm{I}^{+}\right)-D_{0}^{0}\left(\mathrm{I}_{2}^{+}\right)$. This would result in a minimum TKER of $1.97 \mathrm{eV}\left(\mathrm{X}^{2} \Pi_{3 / 2, g}\right)$ and $2.62 \mathrm{eV}\left(\mathrm{X}^{2} \Pi_{1 / 2, g}\right)$ for $266 \mathrm{~nm}$ and $0.71 \mathrm{eV}\left(\mathrm{X}^{2} \Pi_{3 / 2, g}\right)$ and $1.35 \mathrm{eV}\left(\mathrm{X}^{2} \Pi_{1 / 2, g}\right)$ for $365 \mathrm{~nm}$ if no internal excitation of the $\mathrm{I}_{2}^{+}$is assumed, with a dissociation energy of $2.699 \mathrm{eV}$ 
and $2.04 \mathrm{eV}$ for the two spin orbit components of $\mathrm{X}^{2} \Pi_{g}$ respectively.

I could determine three vibrational quanta in the photoelectron spectra, other conventional photoelectron experiments determined a maximum of $\mathrm{v}^{+}=4[13,14]$, which gives an internal excitation energy $\mathrm{E}_{\text {int }}\left(\mathrm{I}_{2}^{+}\right) \approx 0.12 \mathrm{eV}$ for both spin orbit states [8]. Although these values give quite good agreement with the measured values at $365 \mathrm{~nm}$ and the high energy value at $266 \mathrm{~nm}$, the low energy value in this measurement is out of bounds. Its onset is at $2.02 \mathrm{eV}$, and its maximum at $1.74 \mathrm{eV}$, which does not fit a minimum energy of $1.97 \mathrm{eV}$. Arguing on energy grounds only, the most intense part at $1.74 \mathrm{eV}$ of this broad feature matches a process going from $\mathrm{I}_{2}^{+}\left(\mathrm{X}^{2} \Pi_{1 / 2, g}\right) \longrightarrow \mathrm{I}\left({ }^{2} \mathrm{P}_{3 / 2}\right)+\mathrm{I}^{+}\left({ }^{3} \mathrm{P}_{0}\right)$ with a nominal value of $1.74 \mathrm{eV}$. But this should be a sharp peak or, if internal excitation of the $\mathrm{I}_{2}^{+}$is assumed, exhibit a shoulder to the high energy side, but not to the low energy side. Any further electronic excitation of the fragments should result in peaks at the appropriate energies on the low energy side, but not in a continuously decreasing distribution.

Another point is that even if the above described process $\mathrm{I}_{2}^{+}\left(\mathrm{X}^{2} \Pi_{1 / 2, g}\right) \longrightarrow$ $\mathrm{I}\left({ }^{2} \mathrm{P}_{3 / 2}\right)+\mathrm{I}^{+}\left({ }^{3} \mathrm{P}_{0}\right)$ is correct, and in the broad distribution are some contributions hidden that correspond to $\mathrm{I}_{2}^{+}\left(\mathrm{X}^{2} \Pi_{3 / 2, g}\right) \longrightarrow \mathrm{I}\left({ }^{2} \mathrm{P}_{3 / 2}\right)+\mathrm{I}^{+}\left({ }^{3} \mathrm{P}_{0,1}\right)$, why is the overall contribution from $\mathrm{X}^{2} \Pi_{3 / 2, g}$ state in the $266 \mathrm{~nm}$ case so low? From the photoelectron picture we know that ionization to the $\mathrm{X}^{2} \Pi_{3 / 2, g}$ state has the highest probability, and if the picture at $365 \mathrm{~nm}$ shows actually $\mathrm{I}_{2}^{+}$dissociation, it approximately mirrors this ratio. At the nominal TKER corresponding to $\left.\mathrm{I}_{2}^{+}\left(\mathrm{X}^{2} \Pi_{3 / 2, g}\right) \longrightarrow \mathrm{I}^{2} \mathrm{P}_{3 / 2}\right)+\mathrm{I}^{+}\left({ }^{3} \mathrm{P}_{2}\right)$, a process which should have a high intensity actually, since there are plenty of states dissociating to ground state products in $\mathrm{I}_{2}^{+}$, the feature has its cut-off at the high energy side.

Thus I conclude that at least the broad feature in the $266 \mathrm{~nm}$ image must adhere to a different mechanism. Due to the qualitatively similar behaviour in both measurements and the above lack of dissociation from $\mathrm{X}^{2} \Pi_{3 / 2, g}$ in the $266 \mathrm{~nm}$ image, I doubt that any of these wavelength dependent peaks correspond to the subsequent dissociation of $\mathrm{I}_{2}^{+}$by the UV wavelengths.

\subsubsection{Excitation of an intermediate of the neutral}

Also conceivable is a process where the energy is lost by the participation of an intermediate states of the neutral.

The easiest way to produce a difference in the expected TKER and the observed one is that if a bound state is excited, the Frank-Condon window for the subsequent step to the final state may shift slightly in respect to the vertical transition from the ground state to the final state. As discussed 
before, due to this small change, the transition energy is altered by a small amount since the curve is met at a different bond length. But this would result in a small defined offset, not a continuous distribution. This mechanism is shown on the left hand side of fig. 15.7.

But if not only one state is excited, but a full range of states, a continuous distribution may result. This range must cover quite a multitude of states since the distributions observed stretch over an energy up to $1 \mathrm{eV}$.

This range could be obtained with a radiative transition from a bound state to a set of ro-vibrational states. If the range and density of states or ro-vibrational levels is high enough and individual states cannot be resolved, this could produce a distribution which would seem continuous if each of this states results in a slightly different Frank-Condon window which ends in the repulsive, steep part of a dissociative ionic potential. This mechanism is illustrated on the right hand side in fig. 15.7.

So the first, easiest check is if appropriate intermediate states exist. An intermediate state can, roughly speaking, only be excited in a resonant step with a favourable Frank-Condon window. An inspection of the experimental and theoretical data in literature shows that the ${ }^{3} \Pi_{g}\left(1_{g}\right)$ state with a 2341 electron configuration was predicted to lie in the vicinity of $3.4 \mathrm{eV}$, although the calculated vertical transition energy is always slightly higher $(3.4 \mathrm{eV}[11], 3.71 \mathrm{eV}$ [9] and $3.56 \mathrm{eV}$ [15]) than the excitation energy available with $365 \mathrm{~nm}$ wavelength $(3.397 \mathrm{eV})$. This state has so far not been experimentally observed, the direct transition from the ground state is forbidden according to Mulliken [11]. But all calculations agree that the state is dissociative in nature and is predicted to dissociate to ground state products ${ }^{2} \mathrm{P}_{3 / 2}+{ }^{2} \mathrm{P}_{3 / 2}$, thus the assumed model of a bound intermediate cannot be kept in case of the $365 \mathrm{~nm}$ UV wavelength.

Apart from that, only ${ }^{2} \mathrm{P}_{1 / 2}$ can be ionized by one VUV photon, thus the ${ }^{2} \mathrm{P}_{3 / 2}+{ }^{2} \mathrm{P}_{3 / 2}$ fragments should not be detectable in the experiment if this dissociation occurs. Also the same behaviour was seen qualitatively when the photon energy was changed in between $355-368 \mathrm{~nm}$, which would be more likely with a bound state which has the needed ro-vibrational states to allow for a broad absorption and not with a resonant repulsive state, and the $365 \mathrm{~nm}$ wavelength is at the threshold of the predicted vertical excitation energy, thus any longer wavelength should not give the same products.

For the $266 \mathrm{~nm}$ UV wavelength, a similar situation is encountered. It is known that the excitation of $\mathrm{I}_{2}$ at $266 \mathrm{~nm}$ yields dissociation via the $\mathrm{C}^{3} \Sigma^{+}\left(1_{u}\right)$ state to ${ }^{2} \mathrm{P}_{3 / 2}+{ }^{2} \mathrm{P}_{1 / 2}$. The $\mathrm{C}^{3} \Sigma^{+}\left(1_{u}\right)$ state is calculated to lie at $4.54 \mathrm{eV}$ [15] and $4.17 \mathrm{eV}$ [9] and was observed at $4.57 \mathrm{eV} \mathrm{[16])} \mathrm{Although} \mathrm{the} \mathrm{resulting} \mathrm{frag-}$ ment ${ }^{2} \mathrm{P}_{1 / 2}$ could be ionized with one VUV photon, the resulting TKER of $2.18 \mathrm{eV}$, was not experimentally observed. Thus dissociation of the neutral 


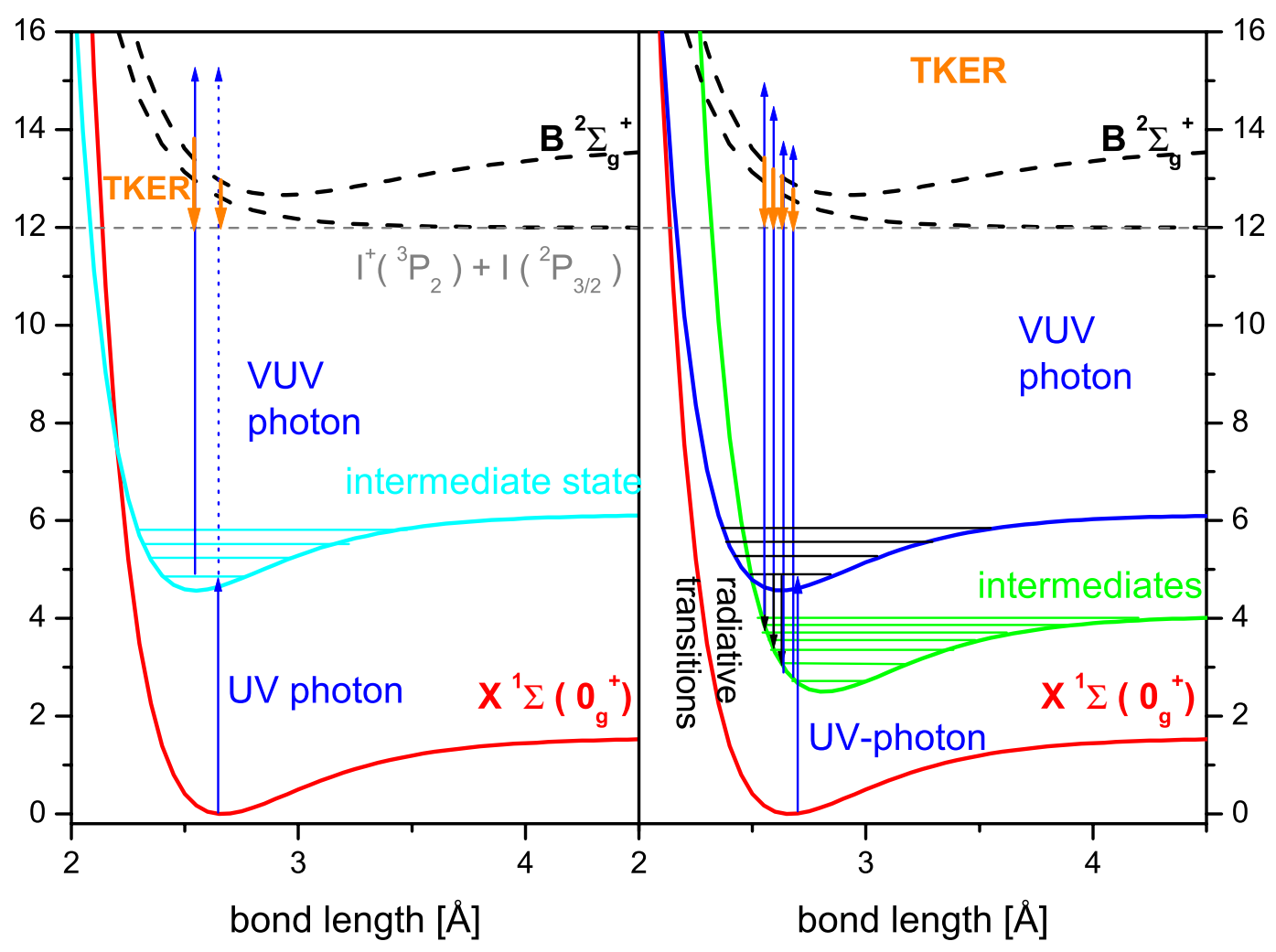

Figure 15.7: Schematic potential curves illustrating the involvement of intermediate states. Dashed lines correspond to ionic states, solid ones to the neutral, grey dashed horizontal lines indicate the ionic asymptotes. Blue arrows represent photons, orange arrows represent the TKER and black arrows radiative transitions. On the left hand, a shift in the Frank-Condon window is shown. The UV photon excites the $I_{2}$ to a bound state. Since the FrankCondon window is more favourable for a different bond length (blue solid arrow), it shifts slightly from the vertical excitation from the neutral ground state $X^{1} \Sigma\left(0_{g}^{+}\right)$(blue dotted arrow), and with it the TKER changes, as given by the orange arrows. On the right hand side the process is shown where from a bound intermediate (blue) radiative transitions to vibrational levels of a lower lying bound state (green) occur. Since the transitions from here go to the repulsive part of the $B^{2} \Sigma_{g}^{+}$potential, small shifts in the Frank-Condon window can create a broad distribution of kinetic energy of the iodine fragments. The orange arrows here illustrate exemplarily the TKER which result from ionization to different parts of the dissociative ionic state.

with UV radiation can be excluded, but also no bound state exist which would justify the mechanism of the continuous shift in the Frank-Condon 
window.

That a step occurs to an intermediate with the VUV photon can be ruled out entirely. If a resonant step is achieved with one VUV photon, the transition goes to a Rydberg state above the first ionization limit. The dominant process from such a Rydberg state, especially a bound one, is autoionizing to $\mathrm{I}_{2}^{+}\left(\mathrm{X}^{2} \Pi_{g}\right)$. Consequently, the discussion in chapter 15.3.1 applies. Other processes which are then possible are discussed above in the section about dissociation to neutrals 15.2.2 and in the chapter about dissociation to ion-pair states 16 . All of these do not provoke a behaviour as seen here.

\subsubsection{Three body decay}

At least for the extremely broad low energy feature in the $266 \mathrm{~nm}$ image, there still is no convincing mechanism which can cause such a behaviour. A different approach would be to assume a three-body decay, in the sense that the electron can take away an undefined amount of energy and thus cause the broad feature. Now a process is needed which allows the electron to do this.

As I mentioned above, if only one UV photon causes the dissociative step, the energy and the fragments are clearly given and do not match the experimental results. If VUV only makes the dissociative step, there will be the same TKER in both images. Thus I conclude that the necessary step is made with absorption of both photons. The energy is then sufficient to excite to a repulsive state of $\mathrm{I}_{2}^{+}$, which would result in the same TKER for both images, but peaked ones. By the combination of two photons even Rydberg states above the first repulsive state $\mathrm{B}^{2} \Sigma_{g}^{+}$can be excited.

\begin{tabular}{|l||c|c|c|c|c|}
\hline $\mathrm{I}_{2}^{+}$three body & ${ }^{3} \mathrm{P}_{2}$ & ${ }^{3} \mathrm{P}_{0}$ & ${ }^{3} \mathrm{P}_{1}$ & ${ }^{1} \mathrm{D}_{2}$ & ${ }^{1} \mathrm{~S}_{0}$ \\
$\mathrm{VUV}+266 \mathrm{~nm}$ & 0 & 0.799 & 0.879 & 1.702 & 2.110 \\
\hline $5 \mathrm{~s}^{2} 5 \mathrm{p}^{5}{ }^{2} \mathrm{P}_{3 / 2} 0$ & 2.867 & 2.068 & 1.989 & 1.166 & - \\
$5 \mathrm{~s}^{2} 5 \mathrm{p}^{5}{ }^{2} \mathrm{P}_{1 / 2} 0.943$ & 1.925 & 1.125 & 1.046 & 0.223 & - \\
\hline \hline \hline $\mathrm{VUV}+355 \mathrm{~nm}$ & 0 & 0.799 & 0.879 & 1.702 & 2.110 \\
\hline $5 \mathrm{~s}^{2} 5 \mathrm{p}^{5}{ }^{2} \mathrm{P}_{3 / 2} 0$ & 1.603 & 0.804 & 0.725 & - & - \\
$5 \mathrm{~s}^{2} 5 \mathrm{p}^{5}{ }^{2} \mathrm{P}_{1 / 2} 0.943$ & 0.661 & - & - & - & - \\
\hline
\end{tabular}

Table 15.4: Nominal maximum TKER values for the three body decay obtained with one VUV and one UV photon. Term values in $[\mathrm{eV}]$

Eventually, this could lead to the behaviour seen in this experiment. As 
it is shown in figure 15.8, the Rydberg state can autoionize to the repulsive ionic curve. Autoionization will then compete with dissociation along the repulsive Rydberg curve. If ionization takes place at a short bond length, most energy will go into the electron. If ionization occurs at a long bond length, most energy will go into the iodine fragments. A similar mechanism was proposed by Unny et al. [3] to explain their feature at very low TKER, but they proposed autoionization to a weakly bound ionic state which allowed the fragments to leave with near to none KER.

That the $\beta$-parameter indicates a mostly parallel transition in the case of high TKER for the iodine fragments, e.g. 1.6 for the maximum value in the $266 \mathrm{~nm}$ case, and decreases slightly with decreasing TKER but still exhibits a rather parallel distribution $(1.0$ for $266 \mathrm{~nm}$ ) is in line with this picture, since the $\beta$-parameter is given by the transition to the upper Rydberg state, and since the system moves along this curve, the character of the transition is mostly preserved. This also means that the lifetime of the Rydberg state cannot be much longer than its average rotational period.

The TKER is then ruled by the following equation:

$$
\begin{array}{r}
E_{\mathrm{TKER}}=E_{h \nu}(V U V)+E_{h \nu}(U V) \\
-E_{\text {int }}(\mathrm{I})-E_{\text {int }}\left(\mathrm{I}^{+}\right)-E_{\text {thresh }}\left(\mathrm{I}^{+}-\mathrm{I}\right)-E_{\text {kin }}\left(e^{-}\right)
\end{array}
$$

with $\mathrm{E}_{\text {thresh }}=11.995 \mathrm{eV}$, threshold energy to produce ground state products $\mathrm{I}\left({ }^{2} \mathrm{P}_{3 / 2}\right)+\mathrm{I}^{+}\left({ }^{3} \mathrm{P}_{2}\right)$ from the neutral molecular ground state $\mathrm{I}_{2}\left(\mathrm{X}^{1} \Sigma\left(0_{g}^{+}\right)\right)$.

The maximum TKER is given when $\mathrm{E}_{\mathrm{kin}}\left(\mathrm{e}^{-}\right)=0$. Values corresponding to different asymptotes are given in table 15.4. The maximum intensity of the feature at low energies in the $266 \mathrm{~nm}$ image lies at an energy $0.26 \mathrm{eV}$ smaller, followed by a slowly decreasing distribution. The maximum energies for all peaks are indicated by arrows in the energy distribution shown in figure 15.2.

If the mechanism holds true for all peaks seen in the images, in both images ground state $\mathrm{I}^{+}\left({ }^{3} \mathrm{P}_{2}\right)+\mathrm{I}\left({ }^{2} \mathrm{P}_{3 / 2}\right)$ and spin orbit excited $\mathrm{I}^{+}\left({ }^{3} \mathrm{P}_{0,1}\right)+4 \mathrm{I}\left({ }^{2} \mathrm{P}_{3 / 2}\right)$ fragments are visible, whereas the latter is much more pronounced in both images.

Apart from that, both images have a peculiarity.

In the $365 \mathrm{~nm}$ image the distribution for the spin orbit excited $\mathrm{I}^{+}\left({ }^{3} \mathrm{P}_{0,1}\right)+$ $\left(\mathrm{I}^{2} \mathrm{P}_{3 / 2}\right)$ channel is more peaked than the corresponding one in the $266 \mathrm{~nm}$ image, as well both peaks are nearer in position to the maximum energy, which is to be expected, since the energy window for the process is narrower. 


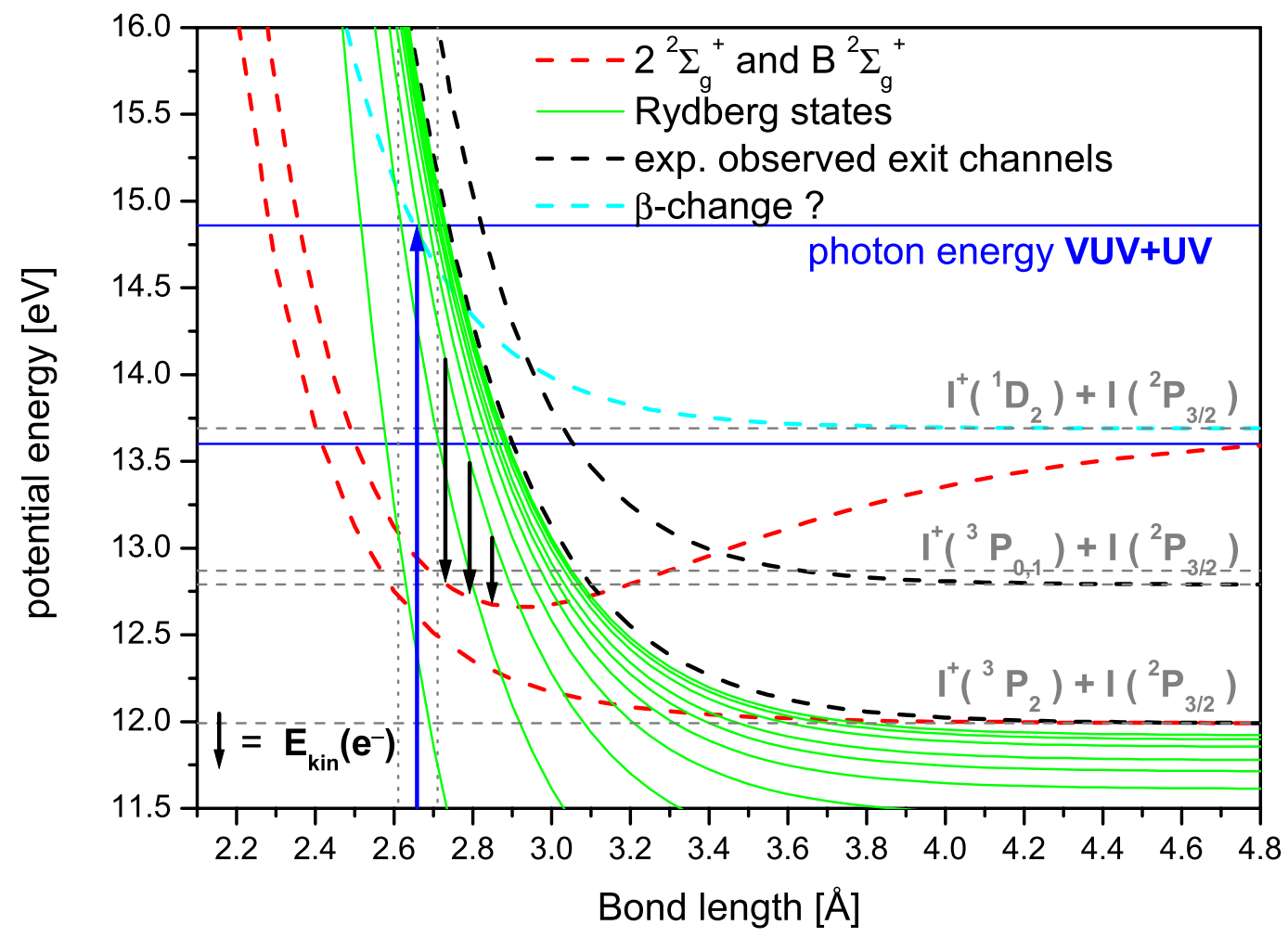

Figure 15.8: Schematic potential curves illustrating interaction of Rydberg states (green lines) with a repulsive curve of the $I_{2}^{+}$. Dashed lines correspond to ionic states, solid ones to the neutral. Grey dashed vertical lines indicate the ionic asymptotes, the grey dotted lines the Frank-Condon window from the ground state. The blue line show the excitation energy achieved with one VUV photon. Blue arrows represent photons, the black arrows represent electrons taking away various amount of kinetic energy. Curves are schematic, but are aimed at depicting the so far assumed correct qualitative behaviour according to Leach [1].

Since it is assumed that the final step in the three body mechanism dissociation is via a repulsive curve one has to consider which one this is. The lowest lying is the $\mathrm{B}^{2} \Sigma_{g}^{+}$state, which would guarantee at least $0.91 \mathrm{eV}$ TKER to the $\mathrm{I}^{+}\left({ }^{3} \mathrm{P}_{2}\right)+\mathrm{I}\left({ }^{2} \mathrm{P}_{3 / 2}\right)$ channel and at least $0.11 \mathrm{eV}$ to the $\mathrm{I}^{+}\left({ }^{3} \mathrm{P}_{0,1}\right)+\mathrm{I}\left({ }^{2} \mathrm{P}_{3 / 2}\right)$ channel. These conditions are met in the experiment and in the $365 \mathrm{~nm}$ case, the TKER measured does not allow for a much higher state, since the distribution with the higher TKER ends around $0.9 \mathrm{eV}$. Hence I believe that in both images the same ionic repulsive curve, the $\mathrm{B}^{2} \Sigma_{g}^{+}$curve, is responsible for the two channels mentioned above. 
But it is improbable that the same part of the Rydberg state is met with a different excitation energy or even that it is the same Rydberg state at all. Therefore the coupling will take place differently and can influence the amount of energy taken away by the electron.

As well it may be that the peak in the $365 \mathrm{~nm}$ picture has some contribution from direct ionization to the $\mathrm{B}^{2} \Sigma_{g}^{+}$state and subsequent decay to the $\mathrm{I}^{+}\left({ }^{3} \mathrm{P}_{2}\right)+\mathrm{I}\left({ }^{2} \mathrm{P}_{3 / 2}\right)$ channel. In the VUV image a peak at $0.78 \mathrm{eV}$ was found which may be attributed to this process. Its $\beta$-parameter is 1.29 and thus not so far from the one found here at 1.63, and the form of the peak here at $0.74 \mathrm{eV}$ is not really discernible due to its proximity to the peak at $0.48 \mathrm{eV}$. Therefore a small contribution cannot be entirely ruled out. As well the possibility of direct ionization to $\mathrm{X}^{2} \Pi_{g}$ states with subsequent dissociation is still a possibility for all other peaks and may or may not contribute to this one.

In the $266 \mathrm{~nm}$ image there is a feature not seen in the $365 \mathrm{~nm}$ spectrum. The overall intensity in the distribution belonging to $\mathrm{I}^{+}\left({ }^{3} \mathrm{P}_{0,1}\right)+\mathrm{I}\left({ }^{2} \mathrm{P}_{3 / 2}\right)$ diminishes to smaller kinetic energies, without any significant change in intensity in between, but there is an obvious change in the $\beta$-parameter. The $\beta$-parameter is 1.59 at the distribution maximum which is as well the highest $\beta$-parameter, and then drops slowly to a smaller value of about 1.0 at the low intensity end of the distribution, but at $1.18 \mathrm{eV}$ the $\beta$-parameter distribution has a dip to a value of 0.70. Apparently here some other mechanism sets in or is superimposed at this TKER value. The value matches either the production to $\mathrm{I}^{+}\left({ }^{1} \mathrm{D}_{2}\right)+\mathrm{I}\left({ }^{2} \mathrm{P}_{3 / 2}\right)\left(\mathrm{E}_{\text {thresh }}=13.69 \mathrm{eV}\right)$ with the electron having zero kinetic energy or the dissociation process $\mathrm{I}_{2}^{+}\left(\mathrm{X}^{2} \Pi_{3 / 2, g}\right) \longrightarrow \mathrm{I}\left({ }^{2} \mathrm{P}_{3 / 2}\right)+\mathrm{I}^{+}\left({ }^{3} \mathrm{P}_{0}\right)$. This could mean that there is a small chance of coupling onto a repulsive curve dissociating to these products. This curve is included in the potential scheme as a light blue dashed line. Since this process is energetically not possible with VUV $+365 \mathrm{~nm}$ photons, it is consistent with the lack of this process in the other image. As well does this process match the process initiated with $212.55 \mathrm{~nm}+$ VUV seen in the VUV images.

Thus the coupling to this repulsive state seems to increase with growing total photon energy and the coupling to the other curve to decrease, since the dominant channels here were not observed in the VUV+212.55 nm spectrum.

\section{Bibliography}

[1] S. Leach. Jour. Phys. Chem., 92:5373, 1988. 
[2] R.P. Tuckett, E. Castellucci, M. Bonneau, G. Dujardin, and S. Leach. Chem. Physics, 92:43, 1985.

[3] S. Unny, Y. Du, L. Zhu, R.J. Gordon, A. Sugita, M. Kawasaki, Y. Matsumi, and Tamar Seidelman. Phys. Rev. Lett., 86:2245, 2001.

[4] L. Minnhagen. Arkiv för Fysik, 21:415, 1962.

[5] P. Venkateswarlu. Can. Jour. Phys., 48:1055, 1970.

[6] T. Ridley, D.A. Beattie, M.C.R. Cockett, K.P. Lawley, and R.J. Donovan. Phys. Cem. - Chem. Physics, 4:1398, 2002.

[7] A. Kvaran, A.J. Yencha, D.K. Kela, R.J. Donovan, and A. Hopkirk. Chem. Phys. Lett., 179:263, 1991.

[8] M.C.R Cockett, R.J. Donovan, and K.P. Lawley. Jour. Chem. Phys., 105:3347, 1996.

[9] W.A. de Jong, L. Visscher, and W.C. Nieuwpoort. Jour. Chem. Phys., 107:9046, 1997.

[10] R.G. McLoughlin, J.D. Morrison, and D.L. Smith. Inter. Jour. Mass Spec. and Ion Proc., 58:201, 1984.

[11] R.S. Mulliken. Jour. Chem. Phys., 55:288, 1971.

[12] A. Hiraya, K. Shobataka, R.J. Donovan, and A. Hopkirk. Jour. Chem. Phys., 88:52, 1988.

[13] B.R. Higginson, D.R. Lloyd, and P.J. Roberts. Chem. Phys. Lett., 19:480, 1973.

[14] H. van Lonkhuyzen and C.A. Lange. Chem. Physics, 89:313, 1984.

[15] C. Teichteil and M. Pelissier. Chem. Physics, 180:1, 1994.

[16] K.P. Huber and G. Herzberg. Molecular spectra and molecular structure; IV. Constants of diatomic molecules. Van Nostrand Reinhold, 1979. 


\section{Chapter 16}

\section{Ion Pair states}

In the VUV experiments, two $\mathrm{I}^{+}$channels were observed which have a corresponding $\mathrm{I}^{-}$channel. These are the the channels with $0.41 \mathrm{eV}$ and $1.27 \mathrm{eV}$ TKER. The images are shown below. The corresponding TKER distributions of the $\mathrm{I}^{+}$and $\mathrm{I}^{-}$channel are shown in figure 16.2. Due to the different voltages needed for the detector when measuring negative particle, the rings of $\mathrm{I}^{-}$are slightly smaller than the corresponding $\mathrm{I}^{+}$rings. But because of their similar $\beta$-parameters which are in contrast to most other $\beta$-parameters found in the $\mathrm{I}^{+}$image and the relative position of the two $\mathrm{I}^{-}$rings, it is obvious which $\mathrm{I}^{+}$rings correspond to them. But because of the change in size, only the $\mathrm{I}^{+}$rings were used to determine the TKER of the two peaks. The I- distribution shown in fig. 16.2 was then scaled to match these TKER values. The factor between these two scales is 1.183.

Because the $\mathrm{I}^{-}$channel exists for these two peaks, these channels do obviously belong to the production of free ion pair states. From the considerations in chapter 3.2.1 it is already known that these two channels are initiated with one VUV photon only, i.e. with $10.20 \mathrm{eV}$ excitation energy. The measured $\beta$ parameter are -0.38 and 0.08 for the $0.41 \mathrm{eV}$ and the $1.27 \mathrm{eV}$ TKER channels respectively. The $0.41 \mathrm{eV}$ channel is 2.1 times more intense than the $1.27 \mathrm{eV}$ channel.

Three channels are energetically possible for the photodissociation of $\mathrm{I}_{2}^{+}$ with one $121.53 \mathrm{~nm}$ photon, but two of the channels are very close in energy and would not be resolved in this experiment. Energetically possible are the dissociation into the ground state of the anion $\mathrm{I}^{-}\left({ }^{1} \mathrm{~S}_{0}\right)$ and and the first three states of the cation $\mathrm{I}^{+}\left({ }^{3} \mathrm{P}_{2,0,1}\right)$. The threshold for ion pair formation is given at a transition energy of $8.935 \mathrm{eV}$ [1]. This is calculated from the dissociation energy of the ground state $\mathrm{I}_{2}(1.542 \mathrm{eV}[2])$, the ionization energy of the atomic I (10.451 eV [3]) and the electron affinity of the I atom (3.059 eV [4]). 

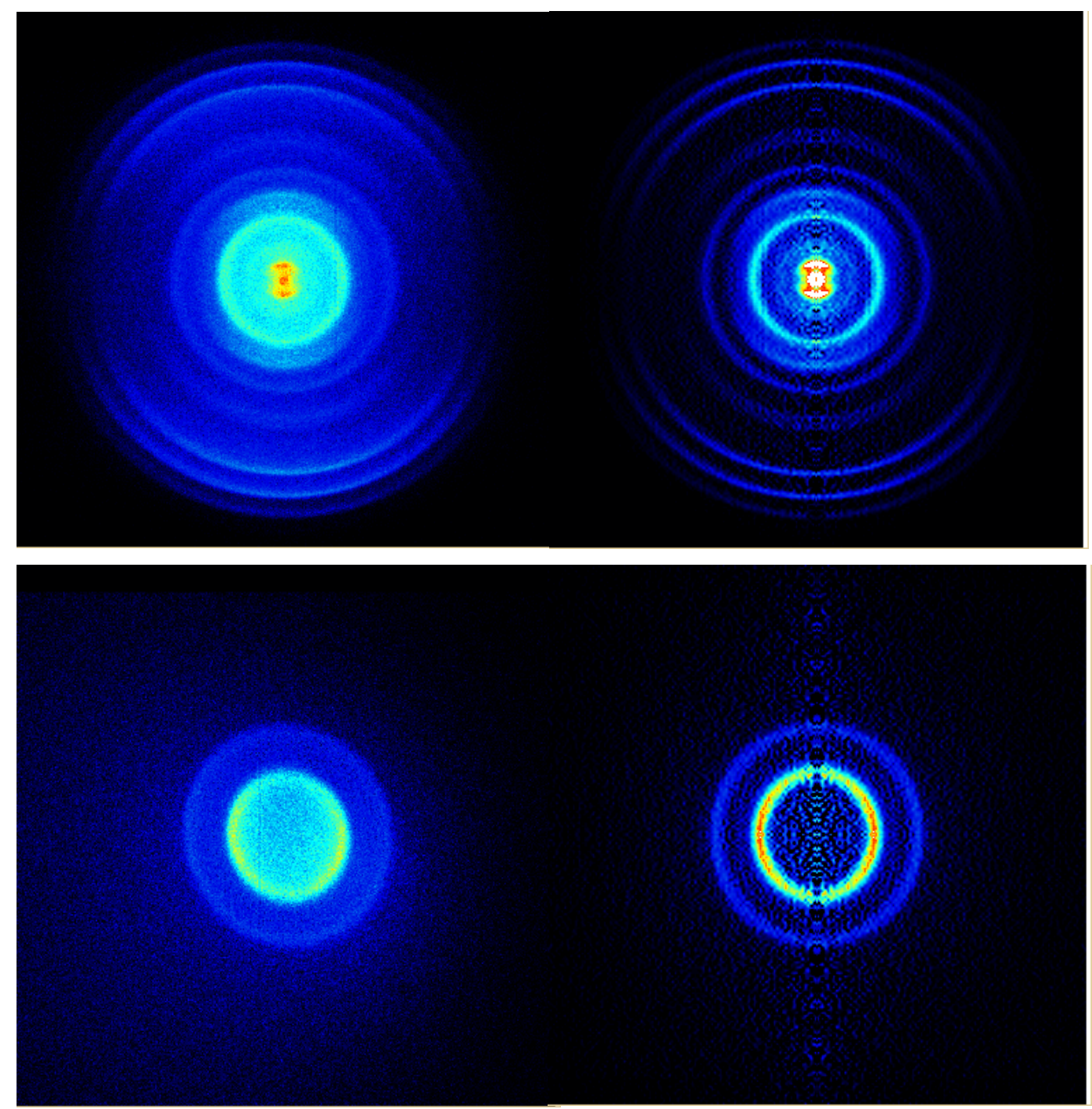

Figure 16.1: Raw and converted images. $I^{+}$images are on top, $I^{-}$images are at the bottom, to the left are the raw images and to the right the corresponding converted images.

The nominal total kinetic energy release of these channels is $1.267 \mathrm{eV}$, $0.467 \mathrm{eV}$ and $0.388 \mathrm{eV}$ respectively. For the dissociation of ion pair states, lowering of the transition energy to the free ion pair state by the extraction field has to be taken into account. This is equivalent to the lowering of the ionization potential by the extracting field for the $\mathrm{I}_{2}+\nu \longrightarrow \mathrm{I}_{2}^{+}+\mathrm{e}^{-}$process. The modification of the transition energy is given by 


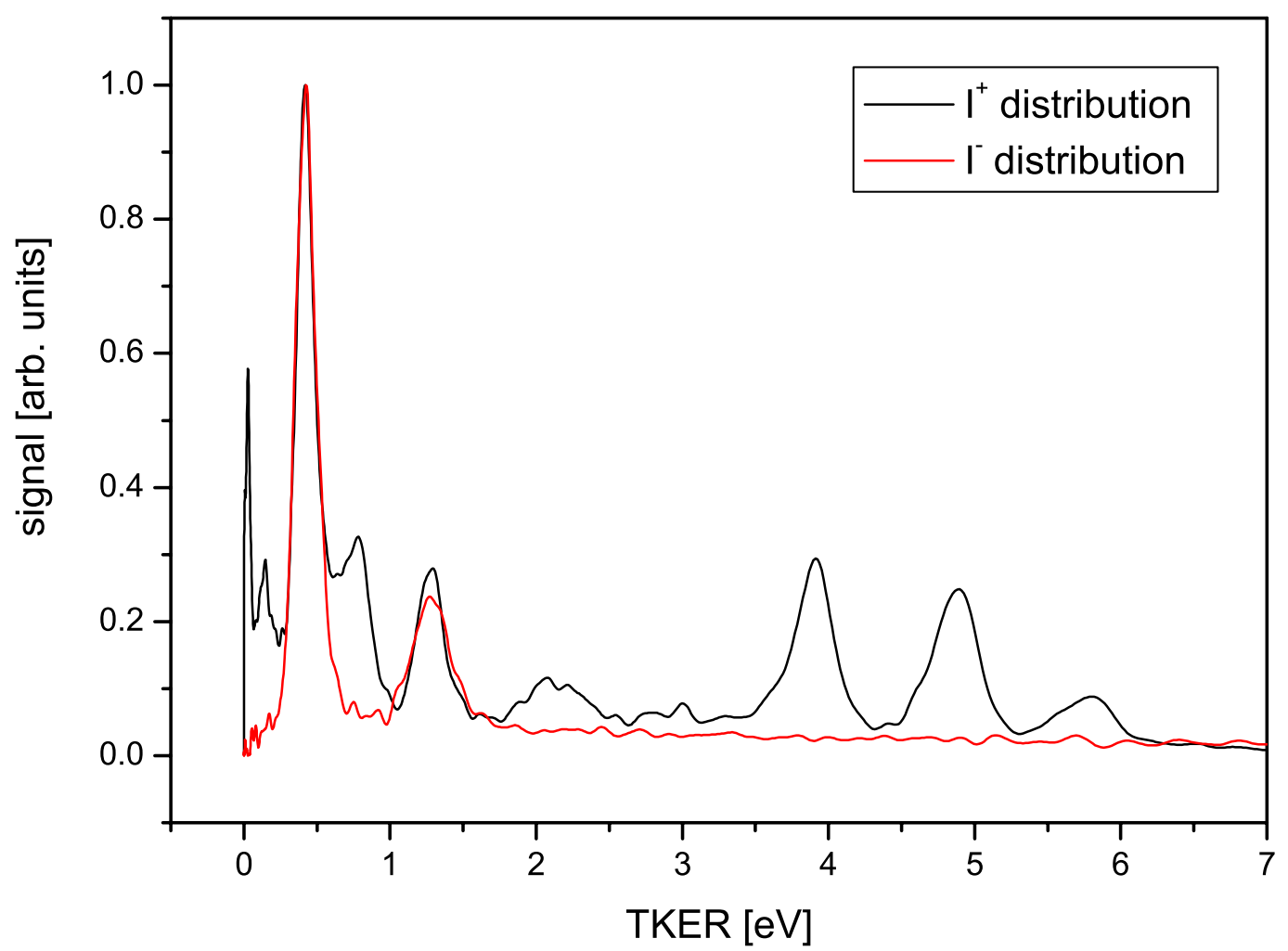

Figure 16.2: TKER distribution of $I^{+}$and $I^{-}$at $121.53 \mathrm{~nm}$ wavelength. The two peaks from the $I^{-}$distribution match the two peaks at 0.41 and $1.27 \mathrm{eV}$ very well and can thus be identified as belonging to the free ion pair state production.

$\Delta \mathrm{E}\left[\mathrm{cm}^{-1}\right] \approx 5 \times \sqrt{V \text { per cm}}$. This gives for the field of the imaging lens of $500 \mathrm{~V}$ per $\mathrm{cm}$ a lowering of roughly $0.01 \mathrm{eV}[1]$. Therefore the above mentioned values should be higher by this value, which gives then $1.277,0.477$, and $0.398 \mathrm{eV}$ TKER. For the higher kinetic energy release, the experimental value fits exactly. On average a value of $1.27 \pm 0.02 \mathrm{eV}$ was obtained with a FWHM width of $0.22 \mathrm{eV}$. The $\beta$-parameter is near zero within experimental error (0.08). The low energy channel with $0.41 \pm 0.02 \mathrm{eV}$ with an average FWHM of $0.14 \mathrm{eV}$ is by $\approx 0.01 \mathrm{eV}$ higher than the expected TKER value of $0.398 \mathrm{eV}$, but still by $0.07 \mathrm{eV}$ lower than the $0.477 \mathrm{eV}$. Thus I conclude that mainly the $\mathrm{I}^{-}\left({ }^{1} \mathrm{~S}_{0}\right)+\mathrm{I}^{+}\left({ }^{3} \mathrm{P}_{1}\right)$ asymptote is populated with only, if at all, small contribution from the $\mathrm{I}^{-}\left({ }^{1} \mathrm{~S}_{0}\right)+\mathrm{I}^{+}\left({ }^{3} \mathrm{P}_{0}\right)$. This view is supported by the fact that the peak is very symmetric and that the $\beta$-parameter does not change significantly within the peak. This channel exhibits a $\beta$-parameter indicating a preferably perpendicular transition. 
The iodine ion pair states has been the subject of many studies within the last years $[5,6,7,8,9,10,11,12,13,14,15,16,1]$. Some of them deal also with the excitation to free ion pair states in the VUV range. Among them is the study by Kvaran et al. [11] who measured an excitation function for the production of free ion pairs in the VUV regime, ranging from $140-120 \mathrm{~nm}$. Although the wavelength used in this experiments is at the very end of the excitation tail, their considerations should in principle apply to the experimentally measured processes here too.

Kvaran et al. obtained an excitation function which could be divided into a longer and shorter wavelength part. The longer wavelengths corresponds to the production of $\mathrm{I}^{-}\left({ }^{1} \mathrm{~S}_{0}\right)$ and $\mathrm{I}^{+}\left({ }^{3} \mathrm{P}_{2}\right)$, since the energy is not high enough for excitation of the other channel. With reaching the threshold energy of the production of the other channel, a second excitation spectrum sets in. The first one could be reproduced when an homogeneous coupling of Rydberg states to the ion pair continuum was assumed. The Rydberg states in question were $[3 / 2]_{c} n \mathrm{p} \pi$ states with $n=9,10$ and 11 . Around the onset of the spectra there would be as well an energetically fitting $[1 / 2]_{c} 7 \mathrm{p} \pi$ state instead of the $[3 / 2]_{c} 9 \mathrm{p} \pi$ state which would be favoured because of the lower principal quantum number, but the inclusion of the latter state makes more sense because it is consistent with the assignment of the other two states. At wavelengths lower than $134.5 \mathrm{~nm}$, the excitation function gets more congested and they propose to include some higher Rydberg states of the same series. In fact, their spectra is nearly perfectly reproduced when including six Rydberg states of this series, but as the authors point out, there will be distortions in the intensity profile by the interaction of Rydberg and ion pair states, therefore the "perfect' reproduction by simulating the excitation function of Rydberg states is probably not very meaningful, since the different coupling terms have to be taken into account.

Below $126 \mathrm{~nm}$ the authors observed a approximately ten times weaker spectrum, which belongs to the excitation $\mathrm{I}^{-}\left({ }^{1} \mathrm{~S}_{0}\right)$ and $\mathrm{I}^{+}\left({ }^{3} \mathrm{P}_{0,1}\right)$ channel. They rate the existence of a structured band near the ionization limit to the $\mathrm{X}^{2} \Pi_{1 / 2}$ as the evidence that the involved Rydberg state do not found on the $[1 / 2] \mathrm{c}$ core state but on the higher ionic core, the $\mathrm{A}^{2} \Pi_{3 / 2}$ state. The Rydberg state appropriate for their spectra is given as the $\mathrm{A}^{2} \Pi_{3 / 2} 8 \mathrm{p} \pi$ state.

Later, Lawley et al. [1] revised the spectra in between $8.85 \mathrm{eV}$ and $9.30 \mathrm{eV}$ but with a higher resolution and came to a somewhat different conclusion. Still, they found the ion pairs to be produced via coupling between Rydberg and ion pair states, but attributed the coupling to different Rydberg 
states. They noticed that their high resolution ion pair spectra agreed within $0.00025 \mathrm{eV}$ with the corresponding absorption spectra. This implies that either all Rydberg states are predissociated by the ion pair continua or that the absorption strength of the Rydberg state is governed by those of the ion pair continua. They decide that the latter possibility is ruled out for the following reasons. First, since there is no structured absorption to high lying bound ion pair states, probably because they are Frank-Condon forbidden (photon outside Frank Condon region for i.p. states $\leftarrow \mathrm{X}{ }^{1} \Sigma\left(0_{g}^{+}\right)$), it is quite unlikely that there is direct transition into the continua. Second, the only ion pair state allowed for one photon absorption is the $\mathrm{D}\left(0_{u}^{+}\right)$state. It is rather improbable that this state alone is responsible for the arising of all ion pair formation in their spectra. They argument that if homogeneous coupling is assumed, there do not exist enough Rydberg states in this region of the same symmetry to explain all observed bands.

All the bands they observed had comparable relative intensities. This is in contrast to the conclusions of Kvaran et al. who believed the ion pair formation to be selective in regard to the Rydberg states. As mentioned above, they attributed the spectra to the coupling of mainly three states Rydberg states onto the ion pair curve. But the most important difference is that due to their higher resolution the origin of the involved bands could be better defined. And in respect to this redetermination, the involved Rydberg states were defined differently. The Rydberg states which dominate the spectra were assigned as $\left[{ }^{2} \Pi_{1 / 2}\right] 4 \mathrm{f}$ states. Contributions from $\left[{ }^{2} \Pi_{1 / 2}\right] 7 \mathrm{p},\left[{ }^{2} \Pi_{1 / 2}\right] 4 \mathrm{f}$ $\left[{ }^{2} \Pi_{3 / 2}\right] 10 \mathrm{p}$ and $\left[{ }^{2} \Pi_{3 / 2}\right] 6 \mathrm{f}$ states gave medium to strong intensity peaks.

The here observed free ion pair states must adhere to the same mechanism, i.e. coupling of a Rydberg state to the free ion pair continuum or continua, but no assumption can be made from these data which ion pair state is actually involved. In table 15.3 known Rydberg states around $10.2 \mathrm{eV}$ are listed, but again, this experiment is not suitable to assign which are likely to be involved, accept that they must be accessible from the neutral ground state $\mathrm{X}^{1} \Sigma\left(0_{g}^{+}\right)$in a one photon step.

The only ion pair state which can be reached with a one photon step from the ground state is the $\mathrm{D}\left(0_{u}^{+}\right)$state, which correlates with $\mathrm{I}^{-}\left({ }^{1} \mathrm{~S}_{0}\right)+\mathrm{I}^{+}\left({ }^{3} \mathrm{P}_{2}\right)$. Because all ion pair states have a bondlength of around $4 \AA$, the FrankCondon window from the ground state is favourable for transitions to the repulsive part above the dissociation limit of the potential curve, which implies that for the high TKER channel in this experiment, direct dissociation into free ion pairs is quite likely. But since the high TKER channel exhibits a nearly isotropic angular distribution, I think that here rather the mechanism of Rydberg states coupling onto the ion pair states applies. Since the 
$\beta$-parameter is near zero, I assume that several Rydberg states contribute.

In comparison the low energy channel exhibits a small negative $\beta$-parameter corresponding to a perpendicular transition moment (-0.38). Therefore, I suggest that this process has one dominating contribution from one Rydberg state with a perpendicular transition, which means that $\Delta \mathrm{J}= \pm 1$.

In general, the ion pair formation at $121.53 \mathrm{~nm}$ is at the high energy tail of cross section for ion pair production. From Kvaran et al. [11] it is known that the main structured part is in between 140 and $133 \mathrm{~nm}$. From the mass spectrum, see chapter 13 it is clear that mainly $\mathrm{I}_{2}^{+}$is produced at $10.20 \mathrm{eV}$.

Also it is known from the experiments at UV+VUV wavelength, that, when the VUV intensity was reduced, the free ion pair products were no longer detectable. But at the same time, with the UV radiation present, atoms from the neutral dissociation of Rydberg states could be detected with a high intensity. Thus it can be concluded that the formation of free ion pair states is a minor channel here.

\section{Bibliography}

[1] K.P. Lawley, A.C. Flexen, R.J. Maier, A. Manck, T. Ridley, and R.J. Donovan. Phys. Cem. - Chem. Physics, 4:1412, 2002.

[2] F. Martin, R. Bacis, S. Churassy, and J. Verges. Jour. Molec. Spec., 116:71, 1986.

[3] S.T. Pratt. Phys. Rev. A, 32:928, 85.

[4] D. Hanstorp and M. Gustafsson. Phys. Rev. B, 25:1773, 1992.

[5] H.P. Grieneisen and R.E. Francke. Chem. Phys. Lett., 88:585, 1982.

[6] C.J. Fecko, M.A. Freedman, and T.A. Stephenson. Jour. Chem. Phys., 116:1361, 2002.

[7] J.P. Perrot, M. Broyer, J. Chevaleyre, and B. Femelat. Jour. Molec. Spec., 98:161, 1983.

[8] M.D. Wheeler, I.R. Lambert, and M.N.R. Ashfold. Chem. Phys. Lett., 229:285, 1994.

[9] U. Heeman, H. Knöckel, and E. Tiemann. Chem. Phys. Lett., 90:17, 1982.

[10] J.C.D. Brand and A.R. Hoy. Can. J. Phys., 60:1209, 1982. 
[11] A. Kvaran, A.J. Yencha, D.K. Kela, R.J. Donovan, and A. Hopkirk. Chem. Phys. Lett., 179:263, 1991.

[12] K.P. Lawley, T. Ridley, Z. Min, P.J. Wilson, M.S.N. Al-Kahali, and R.J. Donovan. Chem. Physics, 197:37, 1995.

[13] P.J. Wilson, T. Ridley, K.P. Lawley, and R.J. Donovan. Chem. Physics, 182:325, 1994.

[14] P.J. Jewsbury, T. Ridley, K.P. Lawley, and R.J. Donovan. Jour. Molec. Spec., 157:33, 1993.

[15] S. Motohiro, A. Umakoshi, and T. Ishiwata. Jour. Molec. Spec., 208:213, 2001.

[16] D. Inard, D. Cerny, M. Nota, R. Bacis, S. Churassy, and V. Skorokhodov. Chem. Physics, 243:305, 1999. 


\section{Chapter 17}

\section{Summary and Outlook}

\subsection{Summary}

In this work, VMI was applied to study decay processes of $\mathrm{I}_{2}$ molecules after photoexcitation above the first ionization threshold. The $\mathrm{I}^{+}$images were at first carefully analyzed in order to assign the processes to the various total excitation energies. Potentials of the iodine cation but also the Rydberg states converging to excited ionic states were considered to explain the experimental results.

In chapter 14, which discusses the results at 10.20 and $20.40 \mathrm{eV}$ total photon energy, most results from dissociative ionization study from Tuckett et al. [1] and the reanalyzation of Leach [2] were corroborated, but amendments to their results were made and because of these, modifications to the so far accepted picture of potentials above the ionization threshold are suggested.

For the absorption of two VUV photons to a dissociative ionic state, a third channel was found with a TKER of $4.88 \mathrm{eV}$ additionally to the known with 3.91 and $5.77 \mathrm{eV}$. This channel was assigned as belonging to the same transition as the $3.91 \mathrm{eV}$ channel, but one of them must couple onto a different dissociative pathway, resulting in a minimum transition energy of $16.88 \mathrm{eV}$, isoenergetic with NeI radiation, excluding the possibility that $3.91 \mathrm{eV}$ dissociates to ground state fragments.

Dissociation via the $\mathrm{B}^{2} \Sigma_{g}^{+}$state was observed at a similar transition energy as other experiments, but in contradiction to their result, here mostly dissociation to the first spin orbit excited state was found.

The pathways to free ion pair states $\mathrm{I}^{+}\left({ }^{3} \mathrm{P}_{2}\right)+\mathrm{I}^{-}\left({ }^{1} \mathrm{~S}_{0}\right)$ and $\mathrm{I}^{+}\left({ }^{3} \mathrm{P}_{0,1}\right)+\mathrm{I}^{-}\left({ }^{1} \mathrm{~S}_{0}\right)$ were corroborated. Here also coupling of high molecular Rydberg states to the ion pair curve was assumed as the main mechanism. 
In chapter 15, a unique process was found in the vicinity of $14 \mathrm{eV}$ photon energy, which was so far undocumented in literature. The transition goes to a high Rydberg state above the first ionization limit which then couples onto a dissociative ionic state, probably the $\mathrm{B}^{2} \Sigma_{g}^{+}$state. The coupling is not very strong and subsequently, the final TKER is determined by the point where the transition to the ionic potential occurs. If the system already moved far along the neutral dissociative curve, the TKER is high, if ionization occurs quickly, the electron takes away most of the energy.

Additionally, a higher lying state must also interfere, which shows up at the higher excitation energy (VUV+266 nm) and is the sole dissociative state observed for excitation with VUV+212.55 $\mathrm{nm}$. The channel comes apparent within the VUV+266 nm distribution because of a different $\beta$-parameter.

Also neutral dissociation to high lying atomic Rydberg states and subsequent ionization was observed.

In the photoelectron images in chapter 13 , ionization to the $\mathrm{X}^{2} \Pi_{3 / 2, g}$ and $\mathrm{X}^{2} \Pi_{1 / 2, g}$ state by one VUV photon was found, and ionization to the $\mathrm{A}^{2} \Pi_{3 / 2, u}$ and $\mathrm{A}^{2} \Pi_{1 / 2, u}$ state with two UV photons, causing high vibrational excitation of the lower state. An additional channel initiated with two UV photons was found, which could be due to the recently found $\mathrm{a}^{4} \Sigma_{u}^{-}$state or, because it is a two photon transition, even belong to the ${ }^{2} \Delta_{u}$ or ${ }^{2} \Sigma_{u}^{+}$states which according to Li and Balasubramanian [3] have both a 2421 configuration and must lie in the vicinity.

\subsection{Outlook}

Most processes have been clarified with a TKER analysis in this work. Additional experiments were performed to determine the excitation energies of these processes. Now, these can be corroborated by probing for the appropriate states or mechanism. In principle, a whole experimental agenda can be outlined. In the case of ionization to a dissociative state, it would be useful to lower the photon energy until the 3.91 and $4.88 \mathrm{eV}$ TKER channels disappear. This gives the exact transition energy and may verify that both belong to the transition to the same initial state, if both channels disappear simultaneously. This requires a tunable source in between 18 and $15 \mathrm{eV}$ if the two photon scheme is kept, which means a wavelength range of $137-165 \mathrm{~nm}$.

The transition energy to the state belonging to the $5.78 \mathrm{eV}$ TKER can be similarly checked, here the wavelength would be around $127 \mathrm{~nm}$. Since these wavelengths are not readily available, these experiments were not performed yet. In the case of the uncertain assignment at low TKER, a similar check could determine which two of the three probable TKER releases really be- 
longs to the dissociation of the $\mathrm{B}^{2} \Sigma_{g}^{+}$state by establishing which two of the three channels do belong together.

In case of the neutral dissociation, it is quite clear that state dependent detection of the atomic Rydberg states is called for. This can also help to clarify the origin of the $0.14 \mathrm{eV}$ peak seen in the VUV experiments.

For the wavelength dependent processes it is interesting to determine at what photon energy the three body decay starts and where it ends and how abrupt the onset of the mechanism is. Of course, here better knowledge of the molecular Rydberg states converging onto an excited ionic state would be convenient.

On the whole, better calculations of the ionic potentials as well as the Rydberg states at higher energies are required to settle the assignments for good. Calculations of the ionic potentials do partly exist, for example when Jong et al. [4] published their relativistic $I_{2}^{+}$potentials, they also obtained a wealth of states dissociating to the ground and spin orbit excited states of atomic and ionic iodine. But as the authors point out, to obtain reasonable potentials for higher states of the iodine molecular cation, extensive correlation treatment and multiconfiguration calculations are needed, thus their results are not exact enough to allow detailed comparison to experimental values. The computational effort to produce exact potentials is still tremendous and hence exact calculations are so far not feasible.

\subsection{Slicing technique}

Although velocity map imaging is now the state-of-the-art technique in photodissociation experiments, it does not mean that it cannot be improved. For example, Wrede et al. [5] designed a better electrostatic lens set-up, minimising spherical and chromatic aberrations by extensive simulations. They give their improvement in the case of chromatic aberration to be a factor of 3.5 in comparison to the standard VMI set-up consisting of three flat plates as it was developed in Nijmegen and used in this work.

Since the method of obtaining the 3D-dimensional distribution of the fragments via an Abel-inversion or reconstruction with basis functions or any other forward or backward convolution is prone to be disturbed by noise, inhomogeneities of the detection or slight problems with focusing the lens, any improvement in the images leads to better inverted images. But even perfect images may still not be converted perfectly because the inversion itself produces artefacts. Therefore many researchers put effort into developing algorithms to reduce these inherent problems, although no method completely 
eliminates these problems.

A survey of these different methods is given in section 11.3.

But what is really the goal of the application of all these algorithms? We want to obtain the 3D-distribution of the fragments, which we can only obtain as an inversion from the 2D-projection if the polarization of the laser is parallel to the detection plane. Usually, this 3D-distribution is represented as a vertical slice through the centre of the newton sphere which contains the same information because of the cylindrical symmetry.

Thus, if we were able to measure this centre slice directly, we could circumvent the reconstruction step and get rid of the artefacts. Plus, we are not limited to the parallel geometry of polarization and detection plane any more. This is useful to explore other aspects of the fragmentation process.

First attempts to establish a slicing technique date back to first attempts on ion imaging itself, but only together with the idea of using focusing electrostatic lenses like they are used in velocity map imaging, its full potential can be tapped.

\subsubsection{Slicing techniques so far}

Toshinori Suzuki and Kenichi Tonokura [6] first followed the style of ion imaging as it was used and developed by Richard Zare and David Chandler [7]. The essence of this method is that the spatial distribution of the fragment is preserved and transferred onto the detector via a homogeneous electric field. In this experiment the dissociation takes place in between two plates with different potentials, where usually one of the plates got a small hole to admit the gas and the other one got a bigger opening which is covered with a very fine grid. This ensures that if the plates are big in diameter compared to the dissociation volume, the fragment only experience a homogeneous field until they leave the acceleration area through the grid. After that, usually a field free drift area follows which allows the spatial distribution of the fragments to expand, before it hits the detector. Because of the distortion by the grids, the resolution of the images is quite limited.

To achieve slicing within this technique, the approach of Suzuki and Tonokura put a time delay between the photolysis and the probe laser. As well was the probe laser moved 1-2 mm downstream of the molecular beam. Depending on the velocity of the molecular beam, resulting in a time delay of a couple of $\mu \mathrm{s}$. This gives the Newton sphere time to expand to a size in the magnitude of $10^{-3} \mathrm{~m}$. Then the probe laser is focused with a cylindrical lens to form a $300 \mu \mathrm{m}$ laser sheet which is used to probe only a slice of the newton sphere. They demonstrated the feasibility of this approach on the dissociation of $\mathrm{NO}_{2}$ and $\mathrm{O}_{2}$ at $355 \mathrm{~nm}$ with subsequent $(1+1)$ - or $(2+1)$-REMPI 
detection respectively. Due to the grids, the resolution was still rather poor and the experiments did not continue.

One attempt was also started in Bielefeld in 1996 [8]. Here, the slicing effect was produced by a very narrow gate on the detector. A special TOF mass spectrometer was constructed using SIMION4.0 software. The electrodes were designed to preserve the spatial distribution as well as possible, especially to prevent the pancaking effect, which means that the spatial distribution is usually crushed or compressed along the TOF axis. Here, the expansion took place in weak field, and the newton sphere passed an additional acceleration field. The experiment were performed on HI detecting the H-atom with $\left(1+1^{\prime}\right)$-REMPI. The main problem here was again that the grids produced a great distortion of the images, as well for some reason the images were of asymmetrical intensity.

The group of Theofanis Kitsopoulos [9] used a similar approach by producing the slice via a very narrow gate on the detector. They overcame the problem concerning the crushing of the Newton sphere by using pulsed extraction. Here, a time delay was put between the production of the ionized fragments and the pulsed extraction of the ions. This allows the Newton sphere to expand prior to extraction. After the extraction grid follows an electrostatic lens and a field free drift region. What happens is that the extension of the sphere decreases and vanishes at a geometric focus and then increases again. This means that when reaching the detector the ion cloud got a reasonable spread along the TOF axis, in their case they could reach a temporal spread of $400 \mathrm{~ns}$ for the whole cloud. By using a gate of $<40 \mathrm{~ns}$ at the detector, they were able to take a slice out of the middle of the distribution. This time lag technique was originally introduced by Wiley and McLaren [10], but they used it to enhance mass resolution by choosing the voltages so that the geometric focus was directly on the detector.

\subsubsection{Slicing and Velocity Map Imaging}

Together with the group of Prof. Dave Parker in Nijmegen, we tried to combine Suzuki's approach of slicing the Newton sphere with a thin laser sheet with the advantages of velocity map imaging. These experiments were all performed in Nijmegen together with André Eppink, Dmitriy Chestakov and Konstantin Vidma.

To combine the two methods, the dissociation took place within the electrostatic lens and probing happened some mm downstream of the molecular beam, also still within the electrostatic lens.

The probing laser was formed into a laser sheet by a cylindrical lens. The sheet had a thickness of approximately $100 \mu \mathrm{m}$. Due to the geometry of the 
apparatus the maximum spatial difference between the two lasers was only a couple of $\mathrm{mm}$, which resulted in a time delay of $\approx 1 \mu \mathrm{s}$. Therefore, the setup is restricted to species, where the probed fragment has sufficient speed after the dissociation so that the Newton sphere reaches at least a diameter which is large compared with the laser sheet thickness. This problem was encountered when first trying to dissociate HI and probe I atoms with slicing techniques. Since the hydrogen atoms take most of the energy because of the mass ratio, the sphere of the Iodine atoms was very small. Other experiments with $\mathrm{CH}_{3} \mathrm{I}$ and $\mathrm{I}_{2}$ proved to be more feasible.

When real slicing is achieved, it is possible then to scan in time through the 3D-distribution by scanning the delay time, starting with a dot or spotlike image, representing the fastest portion of the ions, growing to a ring of maximum diameter representing the centre slice through the distribution and then decreasing again to a smaller spot. These last ions are the slowest with a velocity component which is antiparallel to the direction of the molecular beam. If their recoil speed component in this direction is higher than the beam velocity, these particles do not reach the probing region at all and are therefore lost for detection. As well do the images for the slower parts tend to be more blurred. This is because their velocity gained in the dissociation process is directed backwards to the initial velocity of the parent molecule and also backwards to the acceleration direction, thus they remain longer within the lens with only a low speed, which is roughly the cm-speed minus the speed gained in the dissociation. Thus they suffer more disturbances by fields, as well the molecular beam velocity spread has more influence, since the cm-velocity of the parent molecule is determined by the beam velocity.

The figures 17.1 and 17.2 show examples of the slicing technique. In both cases iodine atoms are probed. To the left, the original images are shown. These contain not only the sliced distribution, but also a projection of the dissociation by the probe wavelength. This is in both cases the smaller ring, due to the longer wavelength of the probe lasers. The dissociation and subsequent ionization by the probe laser of course takes place in the same spot, thus a regular velocity map image results. To make the sharp rings of the slicing technique clearer, from these images an image containing only the velocity map image from the photodissociation by the probe laser is subtracted, resulting in an image which only shows the sliced rings.

The dissociation of $\mathrm{CH}_{3} \mathrm{I}$ is shown in figure 17.1. Dissociation wavelength is $248 \mathrm{~nm}$, probe wavelength is $305.57 \mathrm{~nm}$, which probes the spin orbit excited $\mathrm{I}\left({ }^{2} \mathrm{P}_{1 / 2}\right)$ state. Although $248 \mathrm{~nm}$ is a typical excimer wavelength, it was produced with an Nd-Yag pumped dyelaser, therefore it had a spotsize profile and more important, a $100 \%$ polarization of the laser light, which is necessary to extract further detail about alignment and orientation of the 


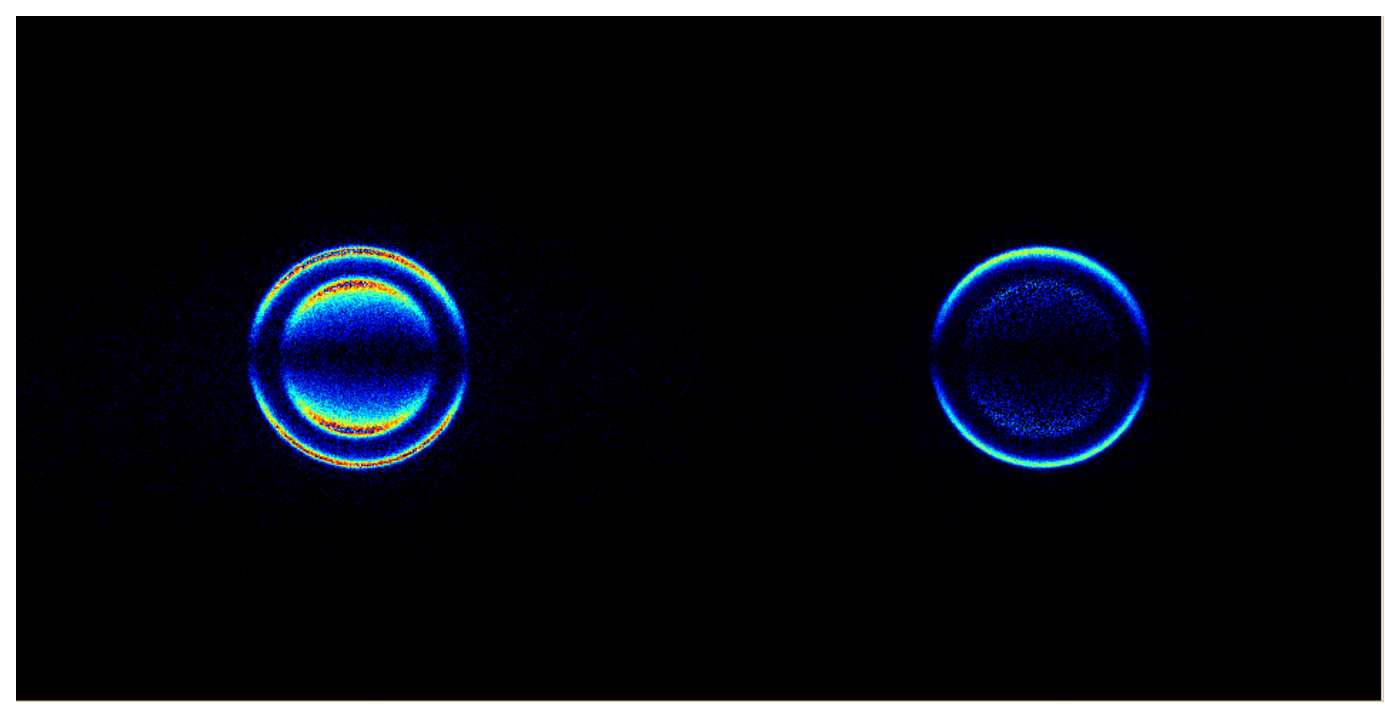

Figure 17.1: Slicing images of $\left.\mathrm{I}^{2} \mathrm{P}_{1 / 2}\right)$ from $\mathrm{CH}_{3} I$. Dissociation wavelength $248 \mathrm{~nm}$, probe wavelength $305.57 \mathrm{~nm}, 1 \mu \mathrm{s}$ delay time. On the left, the original image is shown. The outer ring is the slicing image, the inner is a VMI image of the photodissociation of $\mathrm{CH}_{3} \mathrm{I}$ at probe wavelength. On the right, the same image is shown, but with the dissociation of the probe wavelength subtracted. It can be clearly made out that the outer ring is a real ring, whereas the 2D-projections have more a half-moon character.

atoms.

In figure 17.2 the dissociation of iodine molecule at $266 \mathrm{~nm}$ wavelength is shown. The iodine ground state ${ }^{2} \mathrm{P}_{3 / 2}$ is probed $304.54 \mathrm{~nm}$. It is known that the dissociation of $\mathrm{I}_{2}$ at $266 \mathrm{~nm}$ yields only $\left.\mathrm{I}^{2} \mathrm{P}_{3 / 2}\right)+\mathrm{I}^{*}\left({ }^{2} \mathrm{P}_{1 / 2}\right)$, [11] although two other channels would be energetically allowed, which would give either two ground state atoms or two excited ones. That there is only one channel with ${ }^{2} \mathrm{P}_{3 / 2}$ state is clearly verified by the images, as well that it is a perpendicular transition. Probing the ${ }^{2} \mathrm{P}_{1 / 2}$ state gave the same result. Dissociation is probably achieved via the $\mathrm{C}^{3} \Sigma^{+}\left(1_{u}\right)$ state as assigned by Mulliken.

The sharp rings seen in the figures can be directly evaluated regarding velocity and angular distribution, without using any inversion algorithm.

The original aim of this experiment was not only to establish in principle the slicing technique in combination, but also to obtain information about alignment and orientation effects in the dissociation process. Alignment parameter can be determined by taking a set of images, with the probe and 


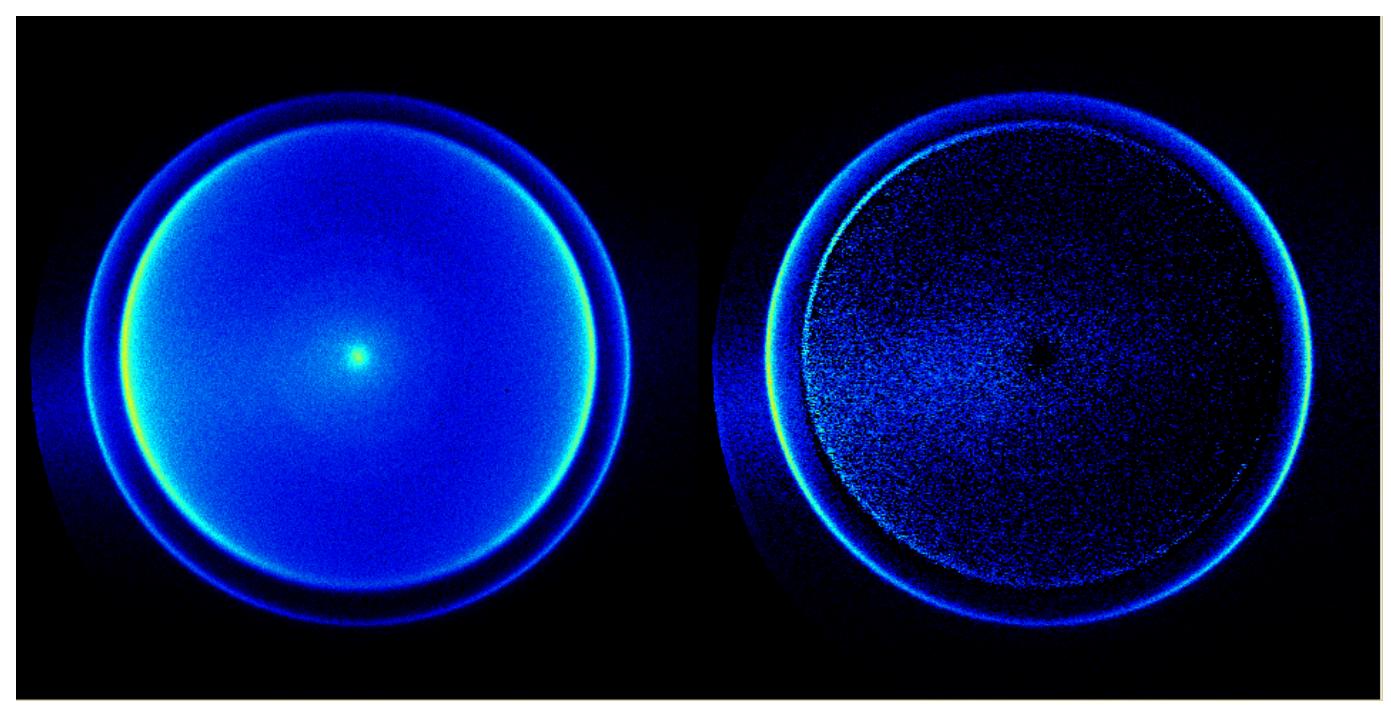

Figure 17.2: Slicing images of $\left.I^{2}{ }^{2} P_{3 / 2}\right)$ from $I_{2}$. Dissociation wavelength $266 \mathrm{~nm}$, probe wavelength $304.54 \mathrm{~nm}$. On the left, the original image is shown, again the inner ring is an ordinary VMI image of the dissociation at probe wavelength. The image on the right has the dissociation from the probe wavelength subtracted. Unfortunately, the image which was subtracted is slightly distorted compared to this one, hence the subtraction is not perfect. Here, the real ringlike character of the outer feature is even clearer.

dissociation laser having both vertical polarization, or both horizontal or both possible mixed combinations.

Slice images allow to measure directly the alignment of photodissociation processes. The effect that photodissociation usually yields photofragments with an anisotropy of the angular distributions. This spatial anisotropy is described with the expression

$$
I(\theta)=\frac{1}{4 \pi}\left[1+\beta P_{2}(\cos \theta)\right]
$$

as explained in chapter 2 .

But not only does the photodissociation process produce spatial anisotropy, very often also an anisotropy of the photofragment angular momentum distribution exists. This influences then the detection of the fragments, since the probing of the fragments is sensitive to the alignment of the angular momentum and the polarization of the probe laser.

If the polarization of the photolysis and probe laser are parallel, the appropriate expression for a process depending on both lasers is an expansion 
of Legendre polynomials of even order. For small $\mathrm{J}$ values, i.e. $\mathrm{J} \leq 3 / 2$, the expansion can be terminated at the fourth order.

$$
I(\theta)^{F G}=\frac{1}{4 \pi}\left[1+\beta_{2}^{F G} P_{2}(\cos \theta)+\beta_{4}^{F G} P_{4}(\cos \theta)\right]
$$

$\mathrm{P}_{4}$ and $\mathrm{P}_{2}$ are the Legendre polynomials second and fourth order, and FG denote the directions of the laser polarization with respect to the detector plane, i.e. perpendicular (z-axis) or parallel (x-axis). Now a set of four images is taken, one with both lasers parallel $(\mathrm{Xx})$, one with both perpendicular $(\mathrm{Zz})$ and two with one parallel and one perpendicular ( $\mathrm{Xz}$ and $\mathrm{Zx}$ ). The $\mathrm{Zz}$ image is actually a calibration image, where any inhomogeneities are not produced by any anisotropy, but by experimental parameters. For example, for these experiments, the Doppler width is not scanned, but the laser bandwidth should be big enough to include all fragments. The different laser intensities within the Doppler width are then reflected in the Zz image and can be used to calibrate all other images.

The intensity along the slice images of the distribution is then obtained by integrating along radial sectors, equivalent to taking them from inverted images. These intensities are calibrated with the $\mathrm{Zz}$ intensity and then fitted to the expression above, 17.2. For any mixed configuration, $\mathrm{Zx}$ and $\mathrm{Xz}, \beta_{4}^{F G}=0$, because any $\mathrm{z}$ geometry does not contribute to the measured anisotropy and the expansion can be truncated at the second order, as the known expansion above for the one photon transition. Because the $\mathrm{Zx}$ and $\mathrm{Xz}$ geometries strongly decouple the two anisotropies, the spatial angular distribution and the angular momentum distribution, and because the $\beta_{2}^{Z x}$ parameter does not depend at all on the spatial angular distribution, but only on the sensitivity of the detection step to the alignment of the photofragments, it is possible to derive the alignment parameters from them. Which alignment parameters are to be included depends on the exact transition.

The group of Kitsopoulos performed some experiments on halogens, demonstrating the value of this method $[12,13]$. For perpendicular transitions from the ground state of the halogens $\mathrm{Br}_{2}$ and $\mathrm{Cl}_{2}$ to the dissociative $\mathrm{C}^{1} \Pi\left(1_{u}\right)$ state at $355 \mathrm{~nm}$ and of the interhalogens $\mathrm{HBr}$ and $\mathrm{HCl}$ to the $\mathrm{A}^{1} \Pi_{1}$ state at $193 \mathrm{~nm}$, giving ground state fragments, they found alignment in all four cases. $\mathrm{Br}_{2}$ fragmentation yielded a maximum alignment and only populated $\mathrm{m}_{j}= \pm 1 / 2$ indicating that $\mathrm{Br}_{2}$ dissociates directly via the $\mathrm{C}^{1} \Pi\left(1_{u}\right)$, whereas $\mathrm{Cl}_{2}$ has a probability of around 0.4 of a non-adiabatic transition to the $\mathrm{A}^{3} \Pi\left(1_{u}\right)$, which resulted in $81 \% \mathrm{~m}_{j}= \pm 1 / 2$ and $19 \% \mathrm{~m}_{j}= \pm 3 / 2$. For the interhalogens it showed that mostly $\mathrm{m}_{j}= \pm 1 / 2$ was populated, although the $\mathrm{A}^{3} \Pi\left(1_{u}\right)$ state correlates with $\mathrm{m}_{j}= \pm 3 / 2$, thus indicating a nonadiabatic transition to the 
$a^{3} \Pi_{1}$ state. The appropriate derivations of the alignment parameters are given in the two articles by Rakitzis and Kitsopoulos [12] and Rakitzis and Kitsopoulos et al. [13]. A full general theory how to obtain the alignment parameters can be taken from the works from Rakitzis [14] and Rakitzis and Zare [15].

In the Nijmegen group of Dave Parker, recently alignment was detected in $\mathrm{I}_{2}$ dissociation in the wavelength regime of $450-510 \mathrm{~nm}$ in the ${ }^{2} \mathrm{P}_{3 / 2}+{ }^{2} \mathrm{P}_{3 / 2}$ channel. A full analysis is still under way. These measurements were performed with combination of slicing with a laser sheet downstream of the molecular beam within the ion lens, as described above [16].

On the whole, we see that slice imaging does not only get rid of the undesirable inversion process but also uncloses a new wealth of details and relatively easy access to alignment effects, which was impressively proven by the research of Rakitzis and Kitsopoulos.

In respect to the work here, due to the overlap of rings especially at small TKER, an inversion free experiment is definitely desirable. The analysis of a possible alignment could also contribute further clarify the involved potentials. To achieve a really solid and exact assignment, better theoretical knowledge of the involved potentials or experimental results as a reference are required, which to my knowledge do not yet exist. But to clarify the symmetry of these states will be a great step forward, which can give valuable insight by comparison to results from the lighter halogens as $\mathrm{Cl}_{2}, \mathrm{Br}_{2}$ and $\mathrm{F}_{2}$. With the knowledge of the involved potentials, some assignments can be further excluded or confirmed, due to transition selection rules.

On the experimental side, a combination of the slicing technique which makes the inversion dispensable together with improved electrostatic lenses specially adapted to the two laser paths needed in the slicing technique, will probably be the best option in the future.

\section{Bibliography}

[1] R.P. Tuckett, E. Castellucci, M. Bonneau, G. Dujardin, and S. Leach. Chem. Physics, 92:43, 1985.

[2] S. Leach. Jour. Phys. Chem., 92:5373, 1988.

[3] Junqing Li and K. Balasubramanian. Jour. Molec. Spec., 138:162, 1989. 
[4] W.A. de Jong, L. Visscher, and W.C. Nieuwpoort. Jour. Chem. Phys., 107:9046, 1997.

[5] E. Wrede, S. Laubach, S. Schulenburg, A. Brown, E.R. Wouters, A.J. Orr-Ewing, and M.N.R. Ashfold. Jour. Chem. Phys., 114:6, 2001.

[6] K. Tonokura and T. Suzuki. Chem. Phys. Lett., 47:1393, 1994.

[7] D. W. Chandler and P. L. Houston. Two-dimensional imaging if state selective photodissociation products detected by multiphoton ionization. Jour. Chem. Phys., 87:1445, 1987.

[8] A. Ross. Entwicklung zweier Flugzeitmassenspektrometer für die Untersuchung von Photodissoziationsprozessen. Diplomarbeit, Universität Bielefeld, Fakultät für Physik, Juli 1997.

[9] C.R. Gebhardt, T.P. Rakitzis, P.C. Samartzis, V. Ladopoulos, and T.N. Kitsopoulos. Review of Scientific Instruments, 72:3848, 2001.

[10] W.C. Wiley and I.L. McLaren. Review of Scientific Instruments, 26:1150, 1955.

[11] R.D. Clear and K.R. Wilson. J. Molec. Spectroscopy, 47:39, 1973.

[12] T.P. Rakitzis and T.N. Kitsopoulos. Jour. Chem. Phys., 116:9228, 2002.

[13] T.P. Rakitzis, P.C. Samartzis, R.L. Toomes, L. Tsigaridas, M. Coriu, D. Chestakov, A.T.J.B. Eppink, D.H. Parker, and T.N. Kitsopoulos. Chem. Phys. Lett., 364:115, 2002.

[14] T.P. Rakitzis. Chem. Phys. Lett., 342:121, 2001.

[15] T.P. Rakitzis and R.N. Zare. Jour. Chem. Phys., 110:3341, 1999.

[16] D. Chestakov. Private communication, 2004. 


\section{List of Figures}

2.1 Newton sphere from reaction . . . . . . . . . . . . . 7

2.2 Newton sphere from photodissociation . . . . . . . . 8

4.1 Principle of a crossed beam reaction . . . . . . . . . . . 22

5.1 Lens position in LIF set-up . . . . . . . . . . . . . . 27

5.2 Vacuum chamber for the $\mathrm{OH}$ source study . . . . . . . . 28

7.1 Permanent flow valve . . . . . . . . . . . . . . 37

7.2 Hydrogen atoms from the urea/hydrogen peroxide compound . 39

7.3 OH LIF spectrum from $\mathrm{H}_{2} \mathrm{O}_{2}$ dissociation, oulsed valve. . . . . 41

7.4 OH LIF spectrum from $\mathrm{H}_{2} \mathrm{O}_{2}$ dissociation, effusive beam . . . 44

7.5 OH LIF spectrum from $\mathrm{HNO}_{3}$ dissociation at $193 \mathrm{~nm} \ldots . . .45$

9.1 Ionization and dissociation thresholds of $\mathrm{I}_{2} \ldots \ldots \ldots$

11.1 Principle of velocity map imaging . . . . . . . . . . . . 63

11.2 Deblurring property of the electrostatic lens . . . . . . . 65

11.3 Velocity map imaging set-up . . . . . . . . . . . . . . 66

$11.4 \mathrm{HI}$ and $\mathrm{I}_{2}$ dissociation images used for calibration. . . . . . . 71

11.5 KER and TKER distributions from the calibration images. . . 72

12.1 Vacuum chamber for $\mathrm{I}_{2}$ dissociation $\ldots \ldots \ldots 77$

13.1 Mass spectrum from $\mathrm{I}_{2}$ experiments . . . . . . . . . . . 82

13.2 Raw and inverted image of photoelectrons from $\mathrm{I}_{2}^{+} \ldots \ldots$. . . 83

13.3 Photoelectron kinetic energy distribution from $\mathrm{I}_{2}^{+} \ldots \ldots$. . . 86

14.1 Images at $121.53 \mathrm{~nm}$ and $212.55 \mathrm{~nm}$ residue wavelengths . . . 96

14.2 TKER at $121.53 \mathrm{~nm}$ and $212.55 \mathrm{~nm}$ residue wavelengths . . . 97

$14.3 \beta$-parameter for rings at $121.53 \mathrm{~nm} \ldots \ldots \ldots 9 . \ldots . \ldots 9$

14.4 TKER distributions at Lyman- $\alpha(\mathrm{D})$ and (H) VUV wavelength 102

14.5 Comparison of the integrated signal of the peaks at $121.53 \mathrm{~nm} 104$ 
14.6 Potentials of $\mathrm{I}_{2}^{+}$involved in the dissociative ionization at $20.40 \mathrm{eV} 111$ 14.7 Modified dissociative potentials of $\mathrm{I}_{2}^{+}$involved at $20.40 \mathrm{eV}$. . . 120

15.1 Images at $121.53+365 \mathrm{~nm}$ and $121.53+266 \mathrm{~nm}$ wavelengths . . 127 15.2 Energy distribution at $121.53+365 \mathrm{~nm}$ and $121.53+266 \mathrm{~nm}$ wavelengths . . . . . . . . . . . . . . . . . . . . . . . . . 128 15.3 Dissociative potentials of $\mathrm{I}_{2}^{+}$reached with VUV+UV wavelengths 131 15.4 Potentials of $\mathrm{I}_{2}$ illustrating dissociation to neutral fragments . 135 15.5 Integrated signal and width of peaks at VUV+UV wavelengths 137 $15.6 \beta$-parameters for rings at VUV+UV wavelengths . . . . . . . . 138 15.7 Potentials of $\mathrm{I}_{2}$ and $\mathrm{I}_{2}^{+}$illustrating involvement of intermediate states . . . . . . . . . . . . . . . . . . . . 142 15.8 Potentials of $\mathrm{I}_{2}$ and $\mathrm{I}_{2}^{+}$illustrating three body decay . . . . . . 145

16.1 Raw and converted images of $\mathrm{I}^{+}$and $\mathrm{I}^{-}$at $121.53 \mathrm{~nm}$. . . . 149 16.2 TKER distribution of $\mathrm{I}^{+}$and $\mathrm{I}^{-}$at $121.53 \mathrm{~nm}$. . . . . . . . 150

17.1 Slicing images of $\left.\mathrm{I}^{2} \mathrm{P}_{1 / 2}\right)$ from $\mathrm{CH}_{3} \mathrm{I} \ldots$. . . . . . . . . . . . 161 17.2 Slicing images of $\left.\mathrm{I}^{2} \mathrm{P}_{3 / 2}\right)$ from $\mathrm{I}_{2} \ldots \ldots$. . . . . . . . . 162 


\section{List of Tables}

6.1 Density of $\mathrm{OH}$ precursor molecules . . . . . . . . . . 32

6.2 Density of $\mathrm{OH}$ radicals in reaction volume . . . . . . . . 33

6.3 Density of reactively produced D atoms . . . . . . . . . . 33

6.4 Absolute number of detected D atoms . . . . . . . . . . . . 34

$7.1 \mathrm{H}_{2} \mathrm{O}_{2}$ photodissociation at different wavelengths $\ldots \ldots . .36$

13.1 Experimental results of the photoelectrons from $\mathrm{I}_{2}^{+} \ldots \ldots 87$

13.2 Calculated states of $\mathrm{I}_{2}^{+}$and their electron configurations . . . . 92

14.1 Experimental results at $121.53 \mathrm{~nm}$ wavelength . . . . . . . 98

14.2 Scaling factor of the integrated signal at Lyman- $\alpha(\mathrm{D})$ and $(\mathrm{H}) 103$

14.3 Dissociation products from $\mathrm{I}_{2}$ at $121.53 \mathrm{~nm} \ldots \ldots \ldots$

14.4 Dissociation products from $\mathrm{I}_{2}^{+}\left(\mathrm{X}^{2} \Pi_{g}\right)$ at $121.53 \mathrm{~nm} \ldots \ldots . . .107$

14.5 Dissociation products from $\mathrm{I}_{2}^{+}\left(\mathrm{A}^{2} \Pi_{3 / 2, u}\right)$ at $121.53 \mathrm{~nm} . \ldots . .108$

14.6 Dissociation products from $\mathrm{I}_{2}^{+}\left(\mathrm{A}^{2} \Pi_{1 / 2, u}\right)$ at $121.53 \mathrm{~nm} . . . . .109$

14.7 Dissociation products from $\mathrm{I}_{2}^{+}\left(\mathrm{A}^{2} \Pi_{1 / 2, u}\right)$ at $121.53 \mathrm{~nm}$, continued. . . . . . . . . . . . . . . . . 110

14.8 Dissociation products from three body decay at $20.40 \mathrm{eV}$ photon energy, low TKER values . . . . . . . . . . . . . . 114

14.9 Dissociation products from $\mathrm{I}_{2}^{+}\left(\mathrm{X}^{2} \Pi_{3 / 2, g}\right)$ at $121.53 \mathrm{~nm}$, high TKER values . . . . . . . . . . . . . . . . 118

14.10Dissociation products from three body decay at $20.40 \mathrm{eV}$ photon energy, high TKER values . . . . . . . . . . . . . . 122

14.11Summary of experimental results at $121.53 \mathrm{~nm} \ldots \ldots . . .123$

14.12Dissociation products from $\mathrm{I}_{2}^{+}\left(\mathrm{X}^{2} \Pi_{g}\right)$ at $212.55 \mathrm{~nm} \ldots \ldots . .124$

14.13Dissociation products from three body decay at $16.03 \mathrm{eV}$ photon energy . . . . . . . . . . . . . . . . 125

15.1 Experimental results at VUV/UV . . . . . . . . . . 129

15.2 Possible designations of the $\mathrm{I}^{*}$ in the dissociation to neutral fragments . . . . . . . . . . . . . . 133 
15.3 Rydberg states with excited $\mathrm{I}_{2}^{+}$cores . . . . . . . . . . . . 134

15.4 Dissociation products from three body decay at 13.60 and

$14.86 \mathrm{eV}$ photon energy . . . . . . . . . . . . . . . 143 



\title{
DANKE!
}

\author{
Dr. Eckart Wrede \\ Dr. André Eppink \\ Prof. Dr. Peter Andresen \\ PD Dr. Dirk Feldmann \\ Dr. Rudolf Böttner \\ Prof. Dr. Markus Sauer \\ Dipl.-Phys. Frank Holtkamp \\ Dipl.-Ing. Stephan Wörmer \\ Dr. Gerd Wiebusch \\ Dipl.-Phys. Gerd Ahlers \\ Rest von D3 \\ Mechanische Werkstatt \\ Elektronik-Werkstatt
}

Dr. Walter Arnold und Isotopenlabor Biologie

Dipl.-Phys. Janine Jäckel 

Hiermit versichere ich, daß ich die Dissertation selbständig und nur mit den zitierten Hilfsmitteln angefertigt habe.

Britta Niederjohann 
